\title{
Flight testing of a remotely piloted vehicle for aircraft parameter estimation purposes
}

\author{
Brad A. Seanor \\ West Virginia University
}

Follow this and additional works at: https://researchrepository.wvu.edu/etd

\section{Recommended Citation}

Seanor, Brad A., "Flight testing of a remotely piloted vehicle for aircraft parameter estimation purposes" (2002). Graduate Theses, Dissertations, and Problem Reports. 2470.

https://researchrepository.wvu.edu/etd/2470

This Dissertation is protected by copyright and/or related rights. It has been brought to you by the The Research Repository @ WVU with permission from the rights-holder(s). You are free to use this Dissertation in any way that is permitted by the copyright and related rights legislation that applies to your use. For other uses you must obtain permission from the rights-holder(s) directly, unless additional rights are indicated by a Creative Commons license in the record and/ or on the work itself. This Dissertation has been accepted for inclusion in WVU Graduate Theses, Dissertations, and Problem Reports collection by an authorized administrator of The Research Repository @ WVU.

For more information, please contact researchrepository@mail.wvu.edu. 


\title{
Flight Testing of a Remotely Piloted Vehicle for Aircraft Parameter Estimation Purposes
}

\author{
Brad A. Seanor \\ Dissertation submitted to the \\ College of Engineering and Mineral Resources \\ at West Virginia University \\ in partial fulfillment of the requirements \\ for the degree of \\ Doctor of Philosophy \\ in \\ Aerospace Engineering
}

Marcello R. Napolitano, Ph.D., Chair

Giampiero Campa, Ph.D.

Gary J. Morris, Ph.D.

Bojan Cukic, Ph.D.

John L. Loth, Ph.D.

Department of Mechanical and Aerospace Engineering

\author{
Morgantown, West Virginia \\ 2002
}

Keywords: Flight Testing, Remotely Piloted Vehicle, Parameter Estimation, Stability and Control Derivatives 


\section{Abstract \\ Flight Testing of a Remotely Piloted Vehicle for Aircraft Parameter Estimation Purposes \\ Brad A. Seanor}

The contribution of this research effort was to show that a reliable RPV could be built, tested, and successfully used for flight testing and parameter estimation purposes, in an academic setting. This was a fundamental step towards the creation of an automated Unmanned Aerial Vehicle (UAV). This research project was divided into four phases. Phase one involved the construction, development, and initial flight of a Remotely Piloted Vehicle (RPV), the West Virginia University (WVU) Boeing 777 (B777) aircraft. This phase included the creation of an onboard instrumentation system to provide aircraft flight data. The objective of the second phase was to estimate the longitudinal and lateral-directional stability and control derivatives from actual flight data for the B777 model. This involved performing and recording flight test maneuvers used for analysis of the longitudinal and lateral-directional estimates. Flight maneuvers included control surface doublets produced by the elevator, aileron, and rudder controls. A parameter estimation program known as pEst, developed at NASA Dryden Flight Research Center (DFRC), was used to compute the off-line estimates of parameters from collected flight data. This estimation software uses the Maximum Likelihood (ML) method with a Newton-Raphson (NR) minimization algorithm. The mathematical model used a traditional static and dynamic derivative buildup. Phase three focused on comparing a linear model obtained from the phase two ML estimates, with linear models obtained from a (i) Batch Least Squares Technique (BLS) and (ii) a technique from the Matlab system identification toolbox. Historically, aircraft parameter estimation has been performed off-line using recorded flight data from specifically designed maneuvers. In recent years, several on-line parameter identification techniques have been evaluated for real-time on-line applications. Along this research line, a novel contribution of this work was to compare the off-line estimation results with results obtained using a recently introduced frequency based on-line estimation method. Specifically, phase four focused on comparing the ML results with a frequency domain based on-line estimation technique. The RPV vehicle and payload was designed and constructed with the combined efforts of WVU researchers, graduate and undergraduate students of the Mechanical and Aerospace Engineering Department, and a private sub-contractor, Craig Aviation. 


\section{Acknowledgements}

I would like to first thank my wonderful wife Mary Jane for her love and support throughout my graduate work. Thank you for being there to get me through everything. Without her support, none of this work would have been possible. I love you. I would like to acknowledge the encouragement and support of my family.

I would like to thank my committee chairman and research advisor Dr. Marcello Napolitano. Your mentoring, guidance, and support throughout my graduate work has been never-ending. I am grateful for the vast research opportunities we have worked on together. You have always been there to guide and support me. Thank You.

I would like to thank committee member Dr. Giampiero Campa for his help and guidance with this research effort. You taught me that experiences with Matlab could be made to be fun and enjoyable.

I would like to acknowledge and thank my committee members Dr. Gary Morris, Dr. Bojan Cukic, and Dr. John Loth for taking time from their busy schedules to review and contribute their thoughts to this research effort.

At this point, I would like to commend and thank all the flight testing members who helped develop the B777. Many thanks to my two pilots, Peter Cooke and John Craig for your time and support in flying the B777. I would like to acknowledge and thank the rest of the flight crew; Srikanth Gururajan, Yu Gu, Benjamin Reid, Frank Nasuti, and Diego Del Gobbo for their hard work helping with the construction, flight testing, and instrumentation issues. I would like to acknowledge and thank Diego for building the signal conditioning unit used with this project. All of your support and hard effort made the B777 project possible. Thank You.

I would like to thank Chuck Coleman, Lee Metheney, and Clifford Judy from the MAE department for their help with equipment and transportation issues.

Last but not the least, I would like to thank all of my research friends, past and present that have served their time down in the depths of the AeroLab. A special thanks goes out to all of you, who made graduate school a fun and enjoyable experience. 


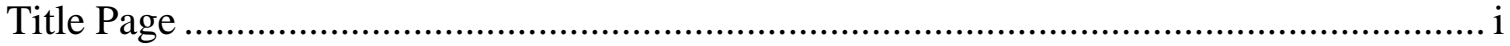

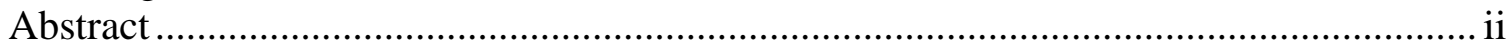

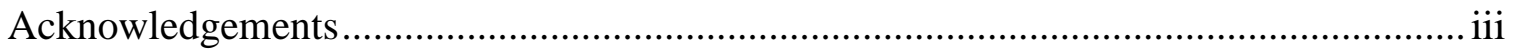

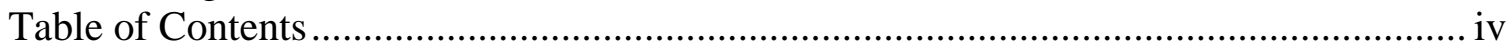

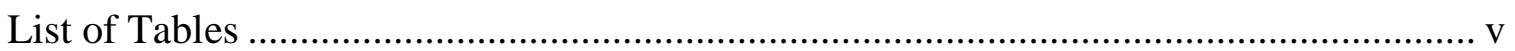

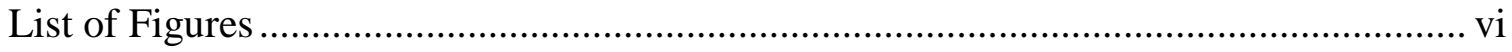

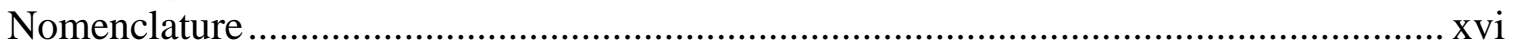

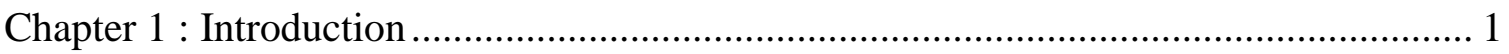

$1.1 \quad$ Research Objectives and B777 Testbed..................................................... 1

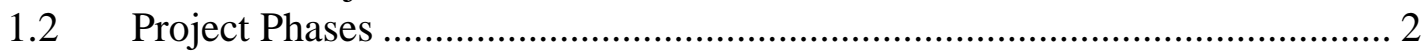

Chapter 2 : Literary Review.................................................................................... 4

2.1 Parameter Estimation Techniques......................................................... 4

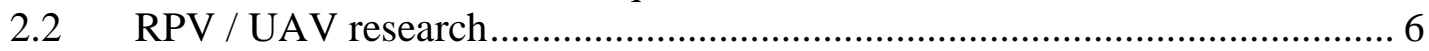

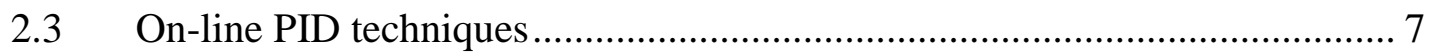

Chapter 3 : Development and Construction of the B777 Aircraft Model........................... 9

3.1 Development of the B777 Aircraft Model ................................................. 9

3.2 B777 Aircraft Model.......................................................................... 11

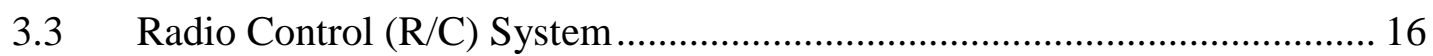

3.4 Propulsion System ………………………....................................... 19

$3.5 \quad$ Instrumentation System ……………………...................................... 21

3.6 Moments of Inertia.............................................................................. 31

Chapter 4 : Aircraft Equations of Motion and Mathematical Modeling............................ 33

$4.1 \quad$ Review of the Equations of Motion ............................................................ 33

4.2 Longitudinal and Lateral-Directional Mathematical Modeling …………..... 38

Chapter 5 : Parameter Estimation Method and pEst Program Operation .......................... 39

5.1 Maximum Likelihood Method and Newton-Raphson Minimization ........... 39

5.2 pEst Program Operation......................................................................... 42

Chapter 6 : Parameter Estimation Results from Collected Flight Data ............................. 44

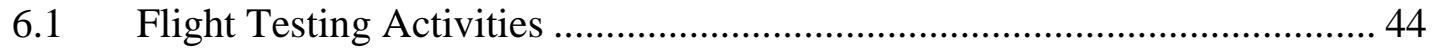

6.2 Flight Testing Maneuvers ...................................................................... 49

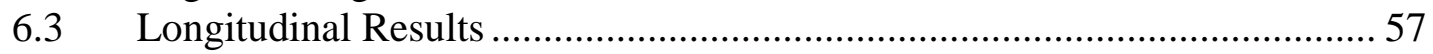

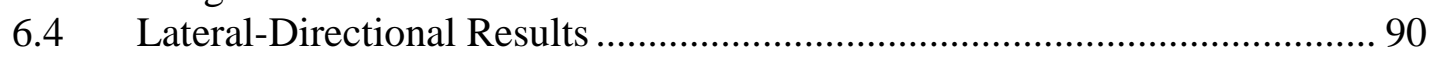

Chapter 7 : State-Variable Modeling of the Aircraft Dynamics ....................................... 153

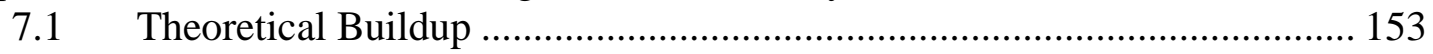

7.2 BLS and System Identification Toolbox Linear Models ............................. 154

7.3 ML Linear Model................................................................................... 158

7.4 Comparison of Longitudinal and Lateral-Directional Linear Models ......... 161

Chapter 8 : On-line (FTR) PID Comparison............................................................ 168

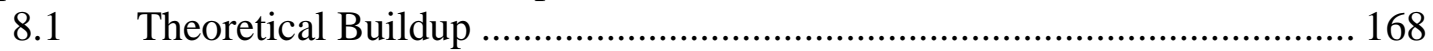

8.2 Longitudinal and Lateral-Directional Results............................................ 172

Chapter 9 : Conclusions and Recommendations ......................................................... 194

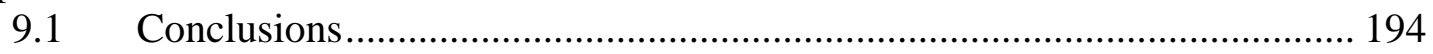

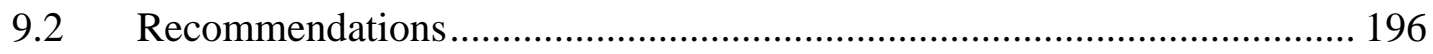

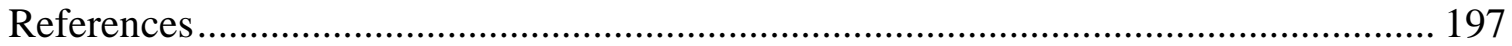

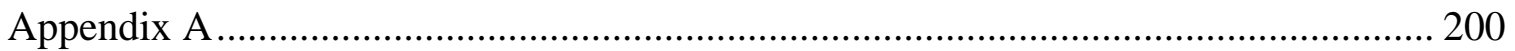




\section{List of Tables}

Table 3.2.1 Mass and geometric characteristics for the B777

Table 3.2.2 Experimentally determined inertia data for the B777

Table 3.2.3 R/C only flight parameters

Table 3.3.1 Current listing of Airtronics servos used for the B777 model

Table 3.3.2 Specifications for various aircraft servos

Table 3.4.1 Engine specifications for the O.S. 91 VF-DR ducted fan engine

Table 3.5.1 Flight parameters with payload

Table 3.5.2 BEI GyroChip ${ }^{\mathrm{TM}}$ Horizon Specifications

Table 3.5.3 CXL04M3 accelerometer specifications

Table 6.2.1 Aircraft configuration for flights \#1, \#2, and \#3

Table 6.2.2 Weight configurations used for preliminary flight tests

Table 6.2.3 Weight configurations used for payload flight tests

Table 6.2.4 Overall number of maneuvers performed during actual flight tests

Table 6.2.5 Longitudinal coefficient table listing for flight test analysis

Table 6.2.6 Lateral-Directional coefficient table listing for flight test analysis

Table 6.2.7 Weight settings for aircraft states (off-line analysis)

Table 7.4.1 Error percentage for longitudinal results

Table 7.4.2 Error percentage for lateral-directional results 


\section{List of Figures}

Figure 3.1.1 WVU B747 model

Figure 3.1.2 WVU B777 model

Figure 3.1.3 Sketch of ground based vehicle test frame

Figure 3.2.1 Aircraft construction and flight testing team

Figure 3.2.2 Fuselage section

Figure 3.2.3 Wing attachment and fuselage assembly

Figure 3.2.4 Engine nacelles and fan assembly

Figure 3.2.5 Wing servo system and fuel bay

Figure 3.2.6 Initial layout of molded parts

Figure 3.3.1 Airtronics Infinity 1000A 10-channel transmitter

Figure 3.3.2 Servo layout for the B777 model

Figure 3.4.1 O.S.91 VF-DR (small head) ducted fan engine

Figure 3.4.2 Field box-starting equipment for propulsion system

Figure 3.4.3 Ramtec ducted fan unit (AeroLoft) with engine in background

Figure 3.5.1 Schematic of instrumentation layout

Figure 3.5.2 Pentium Pro all-in-one CPU card (Advantech)

Figure 3.5.3 Passive backplane

Figure 3.5.4 16-MB flash ram card

Figure 3.5.5 CIO-DAS48-PGL data acquisition card

Figure 3.5.6a Custom computer chassis (front view - fuselage)

Figure 3.5.6b Custom computer chassis (top view)

Figure 3.5.7 Top view of the instrumentation layout for the overall aircraft system

Figure 3.5.8 Signal conditioning interface

Figure 3.5.9 Sensor interface

Figure 3.5.10 BEI GyroChip ${ }^{\mathrm{TM}}$ Horizon (angular rate sensor)

Figure 3.5.11 On board gyro box and accelerometer configuration

Figure 3.5.12 CXL04M3 accelerometer (aluminum version used, far right)

Figure 3.5.13 Pitot-static probe

Figure 3.5.14 Pressure sensor box 
Figure 3.5.15 Alpha and Beta vane

Figure 3.5.16 Angle of Attack and Sideslip vane potentiometers

Figure 3.5.17 Control surface potentiometer

Figure 3.5.18 Elevator and rudder potentiometers

Figure 3.6.1 Pendulum setup (axis-Ix)

Figure 3.6.2 Pendulum setup (axis-Iy)

Figure 4.1.1 Body axis system with forces and moments acting on the aircraft

Figure 4.1.2 Geometric description of aircraft orientation with respect to an Earth fixed inertial axis coordinate system

Figure 4.1.3 Graphical basis for expressing the equations of motion in a polar coordinate form

Figure 5.1.1 Graphical representation of the Newton-Raphson minimization technique

Figure 5.1.2 Block diagram of the Parameter Identification process

Figure 6.1.1 Cradle transportation unit

Figure 6.1.2 Landing gear configuration

Figure 6.1.3 Main gear attachment

Figure 6.1.4 Nose gear strut

Figure 6.1.5 High-speed taxi test

Figure 6.1.6 Return from taxi test

Figure 6.1.7 Flight pattern for the B777 flight testing activities

Figure 6.1.8 Body axis forces and moments acting on the aircraft

Figure 6.2.1 First flight of the B777 aircraft

Figure 6.2.2 High visibility paint scheme

Figure 6.3.1 Sample time history of aircraft parameters for longitudinal Flight 17 ( $\alpha$, pitch rate, normal acceleration, axial acceleration)

Figure 6.3.2 Sample time history of aircraft parameters for longitudinal Flight 17 ( $\beta$, velocity, dynamic pressure, altitude)

Figure 6.3.3 Sample time history of aircraft parameters for longitudinal Flight 17 $\left(\delta_{\mathrm{e}}, \delta_{\mathrm{a}}, \delta_{\mathrm{r}}\right)$

Figure 6.3.4 Comparison of measured and computed time histories from Flight 11, Maneuver 3, longitudinal input ( $\alpha$, pitch rate, normal acceleration, axial acceleration) 
Figure 6.3.5 Comparison of measured and computed time histories from Flight 11, Maneuver 3, longitudinal input ( $\delta_{\mathrm{e}}$, velocity, dynamic pressure, altitude)

Figure 6.3.6 Comparison of measured and computed time histories from Flight 15, Maneuver 3, longitudinal input ( $\alpha$, pitch rate, normal acceleration, axial acceleration)

Figure 6.3.7 Comparison of measured and computed time histories from Flight 15, Maneuver 3, longitudinal input ( $\delta_{\mathrm{e}}$, velocity, dynamic pressure, altitude)

Figure 6.3.8 Comparison of measured and computed time histories from Flight 16, Maneuver 6, longitudinal input ( $\alpha$, pitch rate, normal acceleration, axial acceleration)

Figure 6.3.9 Comparison of measured and computed time histories from Flight 16, Maneuver 6 , longitudinal input ( $\delta_{\mathrm{e}}$, velocity, dynamic pressure, altitude)

Figure 6.3.10 Comparison of measured and computed time histories from Flight 17, Maneuver 3, longitudinal input ( $\alpha$, pitch rate, normal acceleration, axial acceleration)

Figure 6.3.11 Comparison of measured and computed time histories from Flight 17, Maneuver 3, longitudinal input ( $\delta_{\mathrm{e}}$, velocity, dynamic pressure, altitude)

Figure 6.3.12 Comparison of measured and computed time histories from Flight 18, Maneuver 9, longitudinal input ( $\alpha$, pitch rate, normal acceleration, axial acceleration)

Figure 6.3.13 Comparison of measured and computed time histories from Flight 18, Maneuver 9, longitudinal input ( $\delta_{\mathrm{e}}$, velocity, dynamic pressure, altitude)

Figure 6.3.14 Off-line estimation results of $\mathrm{C}_{\mathrm{Norm}_{\mathrm{O}}}$ and $\mathrm{C}_{\mathrm{m}_{\mathrm{O}}}$ for longitudinal Flights 11-13, 15, 16, 17, and 18 versus velocity

Figure 6.3.15 Off-line estimation results of $\mathrm{C}_{\mathrm{Norm}_{\mathrm{O}}}$ and $\mathrm{C}_{\mathrm{m}_{\mathrm{O}}}$ for longitudinal Flights $11-13,15,16,17$, and 18 versus angle of attack

Figure 6.3.16 Off-line estimation results of $\mathrm{C}_{\mathrm{Norm}_{\alpha}}$ and $\mathrm{C}_{\alpha}$ for longitudinal Flights 11-13, 15, 16, 17, and 18 versus velocity

Figure 6.3.17 Off-line estimation results of $\mathrm{C}_{\mathrm{Norm}_{\alpha}}$ and $\mathrm{C}_{\mathrm{m}_{\alpha}}$ for longitudinal Flights $11-13,15,16,17$, and 18 versus angle of attack

Figure 6.3.18 Off-line estimation results of $\mathrm{C}_{\mathrm{Norm}_{\mathrm{q}}}$ and $\mathrm{C}_{\mathrm{q}}$ for longitudinal Flights 11-13, 15, 16, 17, and 18 versus velocity

Figure 6.3.19 Off-line estimation results of $\mathrm{C}_{\mathrm{Norm}_{\mathrm{q}}}$ and $\mathrm{C}_{\mathrm{m}}$ for longitudinal Flights 11-13, 15, 16, 17, and 18 versus angle of attack 
Figure 6.3.20 Off-line estimation results of $\mathrm{C}_{\mathrm{Norm}_{\mathrm{e}_{\mathrm{e}}}}$ and $\mathrm{C}_{\mathrm{m}} \delta_{\mathrm{e}}$ for longitudinal Flights $11-13,15,16,17$, and 18 versus velocity

Figure 6.3.21 Off-line estimation results of $\mathrm{C}_{\mathrm{Norm}_{\mathrm{e}_{\mathrm{e}}}}$ and $\mathrm{C}_{\mathrm{m}_{\mathrm{e}}}$ for longitudinal Flights $11-13,15,16,17$, and 18 versus angle of attack

Figure 6.3.22 Off-line estimation results of $\mathrm{C}_{\mathrm{A}_{\mathrm{O}}}$ and $\mathrm{C}_{\mathrm{A}_{\alpha}}$ for longitudinal Flights $11-13,15,16,17$, and 18 versus velocity

Figure 6.3.23 Off-line estimation results of $\mathrm{C}_{\mathrm{A}_{\mathrm{O}}}$ and $\mathrm{C}_{\mathrm{A}_{\alpha}}$ for longitudinal Flights 11-13, 15, 16, 17, and 18 versus angle of attack

Figure 6.3.24 Off-line estimation results of $\mathrm{C}_{\mathrm{A}_{\mathrm{q}}}$ and $\mathrm{C}_{\mathrm{A}} \delta_{\mathrm{e}}$ for longitudinal Flights $11-13,15,16,17$, and 18 versus velocity

Figure 6.3.25 Off-line estimation results of $\mathrm{C}_{\mathrm{A}_{\mathrm{q}}}$ and $\mathrm{C}_{\mathrm{A}_{\delta_{\mathrm{e}}}}$ for longitudinal Flights $11-13,15,16,17$, and 18 versus angle of attack

Figure 6.3.26 Off-line estimation results of $\mathrm{C}_{\mathrm{Z}_{\mathrm{O}}}$ and $\mathrm{C}_{\mathrm{Z}_{\alpha}}$ for longitudinal Flights $11-13,15,16,17$, and 18 versus velocity

Figure 6.3.27 Off-line estimation results of $\mathrm{C}_{\mathrm{Z}_{\mathrm{O}}}$ and $\mathrm{C}_{\mathrm{Z}_{\alpha}}$ for longitudinal Flights $11-13,15,16,17$, and 18 versus angle of attack

Figure 6.3.28 Off-line estimation results of $\mathrm{C}_{\mathrm{Z}_{\mathrm{q}}}$ and $\mathrm{C}_{\mathrm{Z}_{\delta_{\mathrm{e}}}}$ for longitudinal Flights $11-13,15,16,17$, and 18 versus velocity

Figure 6.3.29 Off-line estimation results of $\mathrm{C}_{\mathrm{Z}_{\mathrm{q}}}$ and $\mathrm{C}_{\mathrm{Z}_{\delta_{\mathrm{e}}}}$ for longitudinal Flights $11-13,15,16,17$, and 18 versus angle of attack

Figure 6.4.1 Sample time history of aircraft parameters for lateral-directional Flight C ( $\beta$, roll rate, yaw rate, lateral acceleration)

Figure 6.4.2 Sample time history of aircraft parameters for lateral-directional Flight C ( $\alpha$, velocity, dynamic pressure, altitude)

Figure 6.4.3 Sample time history of aircraft parameters for lateral-directional Flight C $\left(\delta_{\mathrm{e}}, \delta_{\mathrm{a}}, \delta_{\mathrm{r}}\right)$

Figure 6.4.4 Comparison of measured and computed time histories from Flight A, Maneuver 8, lateral-directional input ( $\beta$, roll rate, yaw rate)

Figure 6.4.5 Comparison of measured and computed time histories from Flight A, Maneuver 8, lateral-directional input (lateral acceleration, $\delta_{\mathrm{a}}, \delta_{\mathrm{r}}$ ) 
Figure 6.4.6 Comparison of measured and computed time histories from Flight A, Maneuver 8, lateral-directional input (velocity, dynamic pressure, altitude)

Figure 6.4.7 Comparison of measured and computed time histories from Flight B, Maneuver 8, lateral-directional input (roll rate, yaw rate, lateral acceleration)

Figure 6.4.8 Comparison of measured and computed time histories from Flight B, Maneuver 8, lateral-directional input $\left(\delta_{\mathrm{a}}, \delta_{\mathrm{r}}\right)$

Figure 6.4.9 Comparison of measured and computed time histories from Flight $C$, Maneuver 7, lateral-directional input ( $\beta$, roll rate, yaw rate)

Figure 6.4.10 Comparison of measured and computed time histories from Flight $\mathrm{C}$, Maneuver 7, lateral-directional input (lateral acceleration, $\delta_{\mathrm{a}}, \delta_{\mathrm{r}}$ )

Figure 6.4.11 Comparison of measured and computed time histories from Flight $\mathrm{C}$, Maneuver 7, lateral-directional input (velocity, dynamic pressure, altitude)

Figure 6.4.12 Comparison of measured and computed time histories from Flight $D$, Maneuver 6, lateral-directional input ( $\beta$, roll rate, yaw rate)

Figure 6.4.13 Comparison of measured and computed time histories from Flight D, Maneuver 6, lateral-directional input (lateral acceleration, $\delta_{\mathrm{a}}, \delta_{\mathrm{r}}$ )

Figure 6.4.14 Comparison of measured and computed time histories from Flight D, Maneuver 6, lateral-directional input (velocity, dynamic pressure, altitude)

Figure 6.4.15 Comparison of measured and computed time histories from Flight $\mathrm{E}$, Maneuver 10, lateral-directional input ( $\beta$, roll rate, yaw rate)

Figure 6.4.16 Comparison of measured and computed time histories from Flight $\mathrm{E}$, Maneuver 10, lateral-directional input (lateral acceleration, $\delta_{\mathrm{a}}, \delta_{\mathrm{r}}$ )

Figure 6.4.17 Comparison of measured and computed time histories from Flight E, Maneuver 10, lateral-directional input (velocity, dynamic pressure, altitude)

Figure 6.4.18 Flight $B$ maneuvers vs. $\mathrm{C}_{\mathrm{l}_{\mathrm{O}}}$ and $\mathrm{C}_{\mathrm{n}_{\mathrm{O}}}$ off-line estimation results

Figure 6.4.19 Flight B maneuvers vs. $\mathrm{C}_{\mathrm{l}_{\mathrm{p}}}$ and $\mathrm{C}_{\mathrm{n}_{\mathrm{p}}}$ off-line estimation results

Figure 6.4.20 Flight B maneuvers vs. $\mathrm{C}_{\mathrm{r}_{\mathrm{r}}}$ and $\mathrm{C}_{\mathrm{n}_{\mathrm{r}}}$ off-line estimation results

Figure 6.4.21 Flight B maneuvers vs. $\mathrm{C}_{\delta_{\mathrm{a}}}$ and $\mathrm{C}_{\mathrm{n}_{\mathrm{a}}}$ off-line estimation results

Figure 6.4.22 Flight B maneuvers vs. $\mathrm{C}_{\delta_{\delta_{\mathrm{r}}}}$ and $\mathrm{C}_{\mathrm{n}_{\mathrm{r}_{\mathrm{r}}}}$ off-line estimation results

Figure 6.4.23 Flight B maneuvers vs. $\mathrm{C}_{\mathrm{y}_{\mathrm{o}}}$ and $\mathrm{C}_{\mathrm{y}_{\mathrm{p}}}$ off-line estimation results 
Figure 6.4.24 Flight B maneuvers vs. $\mathrm{C}_{\mathrm{y}_{\mathrm{r}}}$ and $\mathrm{C}_{\mathrm{y} \delta_{\mathrm{a}}}$ off-line estimation results

Figure 6.4.25 Flight $\mathrm{B}$ maneuvers vs. $\mathrm{C}_{\mathrm{y} \delta_{\mathrm{r}}}$ off-line estimation results

Figure 6.4.26 Off-line estimation results of $\mathrm{C}_{\mathrm{l}_{\mathrm{O}}}$ and $\mathrm{C}_{\mathrm{n}_{\mathrm{O}}}$ for lateral-directional Flights A, B, C, D, and E versus velocity

Figure 6.4.27 Off-line estimation results of $\mathrm{C}_{\mathrm{l}_{\mathrm{O}}}$ and $\mathrm{C}_{\mathrm{n}_{\mathrm{O}}}$ for lateral-directional Flights A, B, C, D, and E versus sideslip angle

Figure 6.4.28 Off-line estimation results of $\mathrm{C}_{\mathrm{l}_{\mathrm{O}}}$ and $\mathrm{C}_{\mathrm{n}_{\mathrm{O}}}$ for lateral-directional Flights A, B, C, D, and E versus velocity (minus selected $\delta_{\mathrm{r}}$ inputs)

Figure 6.4.29 Off-line estimation results of $\mathrm{C}_{\mathrm{l}_{\mathrm{O}}}$ and $\mathrm{C}_{\mathrm{n}_{\mathrm{O}}}$ for lateral-directional Flights A, B, C, D, and E versus sideslip angle (minus selected $\delta_{\mathrm{r}}$ inputs)

Figure 6.4.30 Off-line estimation results of $\mathrm{C}_{\mathrm{l}_{\beta}}$ and $\mathrm{C}_{\mathrm{n}_{\beta}}$ for lateral-directional Flights A, B, C, D, and E versus velocity

Figure 6.4.31 Off-line estimation results of $\mathrm{C}_{\mathrm{l}_{\beta}}$ and $\mathrm{C}_{\mathrm{n}_{\beta}}$ for lateral-directional Flights A, B, C, D, and E versus sideslip angle

Figure 6.4.32 Off-line estimation results of $\mathrm{C}_{\beta}$ and $\mathrm{C}_{\mathrm{n}_{\beta}}$ for lateral-directional Flights A, B, C, D, and E versus velocity (minus selected $\delta_{\mathrm{r}}$ inputs)

Figure 6.4.33 Off-line estimation results of $\mathrm{C}_{\beta}$ and $\mathrm{C}_{\mathrm{n}_{\beta}}$ for lateral-directional Flights A, B, C, D, and E versus sideslip angle (minus selected $\delta_{\mathrm{r}}$ inputs)

Figure 6.4.34 Off-line estimation results of $\mathrm{C}_{\mathrm{l}_{\mathrm{p}}}$ and $\mathrm{C}_{\mathrm{n}_{\mathrm{p}}}$ for lateral-directional Flights A, B, C, D, and E versus velocity

Figure 6.4.35 Off-line estimation results of $\mathrm{C}_{\mathrm{l}_{\mathrm{p}}}$ and $\mathrm{C}_{\mathrm{n}_{\mathrm{p}}}$ for lateral-directional Flights A, B, C, D, and E versus sideslip angle

Figure 6.4.36 Off-line estimation results of $\mathrm{C}_{\mathrm{l}_{\mathrm{p}}}$ and $\mathrm{C}_{\mathrm{n}_{\mathrm{p}}}$ for lateral-directional Flights A, B, C, D, and E versus velocity (minus selected $\delta_{\mathrm{r}}$ inputs)

Figure 6.4.37 Off-line estimation results of $\mathrm{C}_{\mathrm{l}_{\mathrm{p}}}$ and $\mathrm{C}_{\mathrm{n}_{\mathrm{p}}}$ for lateral-directional Flights A, B, C, D, and E versus sideslip angle (minus selected $\delta_{\mathrm{r}}$ inputs)

Figure 6.4.38 Off-line estimation results of $\mathrm{C}_{\mathrm{l}_{\mathrm{r}}}$ and $\mathrm{C}_{\mathrm{n}_{\mathrm{r}}}$ for lateral-directional Flights A, B, C, D, and E versus velocity 
Figure 6.4.39 Off-line estimation results of $\mathrm{C}_{\mathrm{l}_{\mathrm{r}}}$ and $\mathrm{C}_{\mathrm{n}_{\mathrm{r}}}$ for lateral-directional Flights A, B, C, D, and E versus sideslip angle

Figure 6.4.40 Off-line estimation results of $\mathrm{C}_{\mathrm{l}_{\mathrm{r}}}$ and $\mathrm{C}_{\mathrm{n}_{\mathrm{r}}}$ for lateral-directional Flights $\mathrm{A}, \mathrm{B}, \mathrm{C}, \mathrm{D}$, and $\mathrm{E}$ versus velocity (minus selected $\delta_{\mathrm{r}}$ inputs)

Figure 6.4.41 Off-line estimation results of $\mathrm{C}_{\mathrm{l}_{\mathrm{r}}}$ and $\mathrm{C}_{\mathrm{n}_{\mathrm{r}}}$ for lateral-directional Flights A, B, C, D, and E versus sideslip angle (minus selected $\delta_{\mathrm{r}}$ inputs)

Figure 6.4.42 Off-line estimation results of $\mathrm{C}_{\mathrm{l}_{\mathrm{a}}}$ and $\mathrm{C}_{\mathrm{n}_{\mathrm{a}_{\mathrm{a}}}}$ for lateral-directional Flights A, B, C, D, and E versus velocity

Figure 6.4.43 Off-line estimation results of $\mathrm{C}_{\delta_{\delta_{a}}}$ and $\mathrm{C}_{\mathrm{n}_{\mathrm{a}_{\mathrm{a}}}}$ for lateral-directional Flights A, B, C, D, and E versus sideslip angle

Figure 6.4.44 Off-line estimation results of $\mathrm{C}_{\delta_{\mathrm{r}}}$ and $\mathrm{C}_{\mathrm{n}_{\mathrm{r}_{\mathrm{r}}}}$ for lateral-directional Flights A, B, C, D, and E versus velocity

Figure 6.4.45 Off-line estimation results of $\mathrm{C}_{\mathrm{l}_{\mathrm{r}}}$ and $\mathrm{C}_{\mathrm{n}_{\mathrm{r}}}$ for lateral-directional Flights A, B, C, D, and E versus sideslip angle

Figure 6.4.46 Off-line estimation results of $\mathrm{C}_{\mathrm{l}_{\mathrm{r}}}$ and $\mathrm{C}_{\mathrm{n}_{\mathrm{r}_{\mathrm{r}}}}$ for lateral-directional Flights $\mathrm{A}, \mathrm{B}, \mathrm{C}, \mathrm{D}$, and $\mathrm{E}$ versus velocity (minus selected $\delta_{\mathrm{r}}$ inputs)

Figure 6.4.47 Off-line estimation results of $\mathrm{C}_{\mathrm{l}_{\delta_{\mathrm{r}}}}$ and $\mathrm{C}_{\mathrm{n}_{\mathrm{r}_{\mathrm{r}}}}$ for lateral-directional Flights $\mathrm{A}, \mathrm{B}, \mathrm{C}, \mathrm{D}$, and $\mathrm{E}$ versus sideslip angle (minus selected $\delta_{\mathrm{r}}$ inputs)

Figure 6.4.48 Off-line estimation results of $\mathrm{C}_{\mathrm{y}_{\mathrm{o}}}$ and $\mathrm{C}_{\mathrm{y} \beta}$ for lateral-directional Flights A, B, C, D, and E versus velocity

Figure 6.4.49 Off-line estimation results of $\mathrm{C}_{\mathrm{y}_{\mathrm{o}}}$ and $\mathrm{C}_{\mathrm{y} \beta}$ for lateral-directional Flights A, B, C, D, and E versus sideslip angle

Figure 6.4.50 Off-line estimation results of $\mathrm{C}_{\mathrm{y}_{\mathrm{o}}}$ and $\mathrm{C}_{\mathrm{y} \beta}$ for lateral-directional Flights A, B, C, D, and E versus velocity (minus selected $\delta_{\mathrm{r}}$ inputs)

Figure 6.4.51 Off-line estimation results of $\mathrm{C}_{\mathrm{y}_{\mathrm{o}}}$ and $\mathrm{C}_{\mathrm{y} \beta}$ for lateral-directional Flights A, B, C, D, and E versus sideslip angle (minus selected $\delta_{\mathrm{r}}$ inputs)

Figure 6.4.52 Off-line estimation results of $\mathrm{C}_{\mathrm{y}_{\mathrm{p}}}$ and $\mathrm{C}_{\mathrm{y}_{\mathrm{r}}}$ for lateral-directional Flights A, B, C, D, and E versus velocity 
Figure 6.4.53 Off-line estimation results of $\mathrm{C}_{\mathrm{y}_{\mathrm{p}}}$ and $\mathrm{C}_{\mathrm{y}_{\mathrm{r}}}$ for lateral-directional Flights A, B, C, D, and E versus sideslip angle

Figure 6.4.54 Off-line estimation results of $\mathrm{C}_{\mathrm{y}_{\mathrm{p}}}$ and $\mathrm{C}_{\mathrm{y}_{\mathrm{r}}}$ for lateral-directional Flights A, B, C, D, and E versus velocity (minus selected $\delta_{\mathrm{r}}$ inputs)

Figure 6.4.55 Off-line estimation results of $\mathrm{C}_{\mathrm{y}_{\mathrm{p}}}$ and $\mathrm{C}_{\mathrm{y}_{\mathrm{r}}}$ for lateral-directional Flights A, B, C, D, and E versus sideslip angle (minus selected $\delta_{\mathrm{r}}$ inputs)

Figure 6.4.56 Off-line estimation results of $\mathrm{C}_{\mathrm{y}_{\delta_{\mathrm{a}}}}$ and $\mathrm{C}_{\mathrm{y} \delta_{\mathrm{r}}}$ for lateral-directional Flights A, B, C, D, and E versus velocity

Figure 6.4.57 Off-line estimation results of $\mathrm{C}_{\mathrm{y} \delta_{\mathrm{a}}}$ and $\mathrm{C}_{\mathrm{y} \delta_{\mathrm{r}}}$ for lateral-directional Flights A, B, C, D, and E versus sideslip angle

Figure 6.4.58 Off-line estimation results of $\mathrm{C}_{\mathrm{y}_{\delta_{\mathrm{a}}}}$ and $\mathrm{C}_{\mathrm{y} \delta_{\mathrm{r}}}$ for lateral-directional Flights A, B, C, D, and E versus velocity (minus selected $\delta_{\mathrm{r}}$ inputs)

Figure 6.4.59 Off-line estimation results of $\mathrm{C}_{\mathrm{y}_{\delta_{\mathrm{a}}}}$ and $\mathrm{C}_{\mathrm{y} \delta_{\mathrm{r}}}$ for lateral-directional Flights A, B, C, D, and E versus sideslip angle (minus selected $\delta_{\mathrm{r}}$ inputs)

Figure 7.2.1 Setup scheme of longitudinal state-space matrices

Figure 7.2.2 Setup scheme of lateral-directional state space matrices

Figure 7.4.1 Simulink validation scheme for longitudinal state-space models

Figure 7.4.2 Simulink validation scheme for lateral-directional state space models

Figure 7.4.3 Comparison of state-space results for the longitudinal case for the BLS (no vel), identification toolbox (id6), and pEst with measured flight data (Flight 18) for normal acceleration (along with $\delta_{\mathrm{e}}$ input shown)

Figure 7.4.4 Comparison of state-space results for the longitudinal case for the BLS (no vel), identification toolbox (id6), and pEst with measured flight data (Flight 18) for $\alpha$ and pitch rate

Figure 7.4.5 Comparison of state-space results for the longitudinal case for the BLS (no phi), identification toolbox (id6), and pEst with measured flight data (Flight $\mathrm{E}$ ) for lateral acceleration (along with $\delta_{\mathrm{a}} \& \delta_{\mathrm{r}}$ inputs shown)

Figure 7.4.6 Comparison of state-space results for the longitudinal case for the BLS (no phi), identification toolbox (id6), and pEst with measured flight data (Flight $\mathrm{E}$ ) for $\beta$, roll, and yaw rate

Figure 8.1.1 Simulink FTR scheme for longitudinal and lateral-directional dynamics 
Figure 8.2.1 Measured time histories used for FTR / pEst comparison for Flight D, Maneuver 4 ( $\delta_{\mathrm{e}}$ input)

Figure 8.2.2 Comparison of FTR and pEst longitudinal coefficients $\mathrm{C}_{\mathrm{Z}}, \mathrm{C}_{\mathrm{m}}$, and $\mathrm{C}_{\mathrm{A}}$ for Flight $\mathrm{D}$, Maneuver 4 ( $\delta_{\mathrm{e}}$ input)

Figure 8.2.3 Measured time histories used for FTR / pEst comparison for Flight D, Maneuver 5 ( $\delta_{\mathrm{a}}$ input)

Figure 8.2.4 Comparison of lateral-directional coefficients $C_{1}$ and $C_{n}$ for Flight D, Maneuver 5 ( $\delta_{\mathrm{a}}$ input)

Figure 8.2.5 Comparison of lateral-directional coefficients $\mathrm{C}_{\mathrm{y}}$ for Flight $\mathrm{D}$, Maneuver 5 ( $\delta_{\mathrm{a}}$ input)

Figure 8.2.6 Measured time histories used for FTR / pEst comparison for Flight E, Maneuver 10 ( $\delta_{\mathrm{a}}$ input)

Figure 8.2.7 Comparison of lateral-directional coefficients $C_{1}$ and $C_{n}$ for Flight E, Maneuver 10 ( $\delta_{\mathrm{a}}$ input)

Figure 8.2.8 Comparison of lateral-directional coefficients $\mathrm{C}_{\mathrm{y}}$ for Flight $\mathrm{E}$, Maneuver 10 ( $\delta_{\mathrm{a}}$ input)

Figure 8.2.9 Measured time histories used for FTR / pEst comparison for Flight D, Maneuver 3 ( $\delta_{\mathrm{r}}$ input $)$

Figure 8.2.10 Comparison of lateral-directional coefficients $C_{1}$ and $C_{n}$ for Flight D, Maneuver 3 ( $\delta_{\mathrm{r}}$ input)

Figure 8.2.11 Comparison of lateral-directional coefficients $\mathrm{C}_{\mathrm{y}}$ for Flight $\mathrm{D}$, Maneuver 3 ( $\delta_{\mathrm{r}}$ input)

Figure 8.2.12 Measured time histories used for FTR / pEst comparison for Flight E, Maneuver 9 ( $\delta_{\mathrm{r}}$ input $)$

Figure 8.2.13 Comparison of lateral-directional coefficients $C_{1}$ and $C_{n}$ for Flight E, Maneuver 9 ( $\delta_{\mathrm{r}}$ input $)$

Figure 8.2.14 Comparison of lateral-directional coefficients $\mathrm{C}_{\mathrm{y}}$ for Flight $\mathrm{E}$, Maneuver 9 ( $\delta_{\mathrm{r}}$ input $)$ 
Figure 8.2.15 Measured flight data time histories used for FTR / pEst comparison for Flight $\mathrm{C}$, Maneuver $3\left(\delta_{\mathrm{a}} \& \delta_{\mathrm{r}}\right.$ input combo)

Figure 8.2.16 Comparison of lateral-directional coefficients $\mathrm{C}_{1}$ and $\mathrm{C}_{\mathrm{n}}$ for Flight $\mathrm{C}$, Maneuver $3\left(\delta_{\mathrm{a}} \& \delta_{\mathrm{r}}\right.$ input combo)

Figure 8.2.17 Comparison of lateral-directional coefficients $\mathrm{C}_{\mathrm{y}}$ for Flight $\mathrm{E}$, Maneuver $3\left(\delta_{\mathrm{a}} \& \delta_{\mathrm{r}}\right.$ input combo)

Figure 8.2.18 Measured flight data time histories used for FTR / pEst comparison for Flight $\mathrm{C}$, Maneuver $6\left(\delta_{\mathrm{a}} \& \delta_{\mathrm{r}}\right.$ input combo $)$

Figure 8.2.19 Comparison of lateral-directional coefficients $\mathrm{C}_{1}$ and $\mathrm{C}_{\mathrm{n}}$ for Flight $\mathrm{C}$, Maneuver $6\left(\delta_{\mathrm{a}} \& \delta_{\mathrm{r}}\right.$ input combo $)$

Figure 8.2.20 Comparison of lateral-directional coefficients $\mathrm{C}_{\mathrm{y}}$ for Flight $\mathrm{E}$, Maneuver $6\left(\delta_{\mathrm{a}} \& \delta_{\mathrm{r}}\right.$ input combo) 


\section{Nomenclature}

$\underline{\text { SYMBOL }} \underline{\text { DESCRIPTION }} \underline{\text { UNIT(S) }}$

English

alt

$a_{n}$

avg

$\mathrm{a}_{\mathrm{x}}$

$a_{y}$

$\mathrm{b}$ or $\mathrm{B}$

$b_{\text {pe }}$

c

$\mathrm{Ci}$

$\mathrm{Cr}$

$\mathrm{Ct}$

$\mathrm{Fi}$

g

K

L

Mi

m

$\mathrm{p}$

P (z)

psi

q

q

R

r

S

$\mathrm{t}$

$\mathrm{T}$

$\mathrm{T}_{\text {pe }}$

V

vel

W

$\mathrm{X}$

X

$\mathrm{y}$

$\mathrm{y}$

Y

$\mathrm{Z}$ 


\section{Greek}

$\alpha$

$\beta$

$\beta$

$\Delta$

$\delta$

$\nabla$

$\theta$

$\theta_{\mathrm{pe}}$

$\xi$

$\phi$

$\sigma$

$\Sigma$

$\psi$

$\omega$

$\underline{\text { Subscripts }}$

A

a

D

e

$\mathrm{k}$

L

1

m

$\mathrm{N}$

n

$\mathrm{r}$

stall

takeoff

wind

$\mathrm{y}$

$\underline{\text { Superscripts }}$

$\mathrm{T}$

.

$-$

Acronyms

$\mathrm{AC}$

BLS

B747

A

angle of attack

$\operatorname{deg}$

sideslip angle

vector of parameters to be estimated

incremental change

control surface deflection

gradient

pitch angle

angular displacement of pendulum

parameter vector to be estimated

roll angle

estimate standard deviation

summation

yaw angle

frequency deg

$---$

$\operatorname{deg}$

$---$

$\operatorname{deg}$

$\operatorname{deg}$

$\operatorname{deg}$

$---$

$\operatorname{deg}$

$\mathrm{rad} / \mathrm{sec}$ axial force

aileron

drag force

elevator

$\mathrm{k}$-th row

lift force

rolling moment

pitching moment

normal force

yawing moment

rudder

stall characteristics

takeoff performance

wind axis

lateral force

transpose

first derivative with respect to time vector quantity

Aerodynamic Center

Batch Least Squares

WVU Boeing 747 model 
B777

BLUE

CG

CPU

CRB

DOF

DFRC

DTFT

EE

EKF

FTR

FTFCS

GetData

ISA

LCD

LS

LWR

$\mathrm{mAh}$

ML

MSE

MSLS

$\mathrm{NiCD}$

$\mathrm{NiMH}$

NN

NR

PCI

PCM

pEst

PID

RAM

$\mathrm{R} / \mathrm{C}$

$\mathrm{RF}$

RHS

RLS

RMS

RPM

RPV

SFDIA

SVD

UAV

WVU
WVU Boeing 777 model

Best Linear Unbiased Estimation

Center of Gravity

Central Processing Unit

Cramer-Rao Bound

Degrees Of Freedom

Dryden Flight Research Center

Discrete Time Fourier Transform

Estimation Error

Extended Kalman Filtering

Fourier Transform Regression

Fault Tolerant Flight Control System

Time History Utility Program

Industry Standard Architecture

Liquid Crystal Display

Least Squares

Locally Weighted Regression

Milliamp Hour

Maximum Likelihood

Mean Square Error

Modified Sequential Least Square

Nickel Cadmium

Nickel Metal Hydride

Neural Networks

Newton-Raphson

Peripheral Component Interconnect

Pulse Code Modulation

Parameter Identification Program

Parameter Identification

Random Access Memory

Radio / Controlled

Radio Frequency

Right Hand Side

Recursive Least Squares

Root Mean Square

Revolutions Per Minute

Remotely Piloted Vehicle

Sensor Failure Detection,

Identification and Accommodation

Singular Value Decomposition

Unmanned Aerial Vehicle

West Virginia University 


\section{Chapter 1}

\section{Introduction}

\subsection{Research Objectives and B777 Testbed}

The main objectives for this work were to design, build, and fly a Remotely Piloted Vehicle (RPV), and then estimate the longitudinal and lateral-directional stability and control derivatives from the actual RPV flight data. The RPV was built at West Virginia University and was named the WVU B777. This research was a very important step towards the creation of a completely automated Unmanned Aerial Vehicle (UAV). After an initial flight testing phase, maneuvers were performed and recorded with an onboard instrumentation system. These maneuvers included independent longitudinal and lateral-directional control surface doublets produced by the elevator, aileron, and rudder controls. A parameter estimation program known as pEst, developed at NASA Dryden Flight Research Center (DFRC), was used to achieve a set of longitudinal and lateral-directional stability and control derivatives from flight test maneuvers. This estimation software employs the Maximum Likelihood (ML) method with a NewtonRaphson (NR) minimization algorithm to find and update a parameter vector containing estimates of the stability and control derivatives, so that a quadratic cost function was minimized. Historically, aircraft parameter estimation has been performed off-line using recorded flight data created from specifically designed maneuvers, but, in recent years, several parameter identification (PID) techniques have been evaluated for real-time online applications. This research effort also compared the off-line estimation results from ML with results from a recently introduced on-line frequency based PID technique. The radio controlled (R/C) B777 aircraft was designed and developed by researchers and students at the Mechanical and Aerospace Engineering Department of West Virginia University, Morgantown, WV. The overall design and construction was a combination of efforts between professors, graduate students, and undergraduates of the Mechanical and Aerospace Engineering Department, and a private sub-contractor, Craig Aviation. 


\subsection{Project Phases}

This research effort was broken down into four particular phases:

1. Initial development and construction of the B777 aircraft for flight testing purposes;

2. Estimation of a set of longitudinal and lateral-directional stability and control derivatives from the collected flight test maneuvers;

3. Evaluation and verification of the parameter estimates obtained from flight data with other off-line PID techniques using linear models;

4. Compare off-line pEst estimates with estimates produced from an on-line frequency based technique.

\section{Phase \#1}

This stage of the work primarily covered the development and construction of the B777 aircraft and instrumentation payload used to acquire flight data. This document will discuss the aircraft equipment and instrumentation system used in the collection of flight test data. Other chapters will discuss a review of the aircraft equations of motion used for the mathematical model; a review of the parameter estimation method used; and specific information about the B777 flight testing activities. For this fact, phase one of this project was broken down into four sub tasks.

\section{Breakdown of Phase \#1 objectives:}

(1) After completion of the B777 construction, begin ground/taxi tests to assess aircraft handling qualities, ground speed, and R/C aircraft systems.

(2) Flight testing the B777 in an "R/C mode only" and assess the aircraft's handling qualities in air, along with assessing the vehicles propulsion system.

(3) Add an "artificial" payload to the B777, representing the weight distribution of a full electronic payload, followed by flight testing of the model. This stage would provide an evaluation of the handling qualities and performance with a "simulated" payload.

(4) Installation of the full electronic payload followed by flight testing at the WVU Jackson's Mill airfield facility. Flight data would be recorded at a sampling rate of $100 \mathrm{~Hz}$ and stored within a 16MB RAM card for post flight downloading. 


\section{Phase \#2}

Phase two focused on performing, recording, and analyzing longitudinal and lateral-directional PID maneuvers to evaluate the estimates using pEst.

\section{Phase \#3}

Phase three focused on comparing a linear model obtained from the phase two average ML estimates, with linear models obtained from a (i) Batch Least Squares Technique (BLS) and (ii) a technique from the Matlab system identification toolbox. One way of accomplishing this task was to create a state-variable model, or a set of A, B, $\mathrm{C}$ and D matrices describing the linear dynamics of the B777 for each method. Thus, three separate decoupled models for the longitudinal and lateral-directional case (total of 6) were created and compared within the simulation results.

\section{Phase \#4}

Phase four focused on comparing the ML results obtained in phase two with a recently developed frequency based on-line estimation technique. The objective of this phase was to see how the off-line ML B777 estimates compared with a frequency based on-line PID technique. 


\section{Chapter 2}

\section{Literary Review}

\subsection{Parameter Estimation Techniques}

Parameter estimation, when related to aircraft systems, allows scientists and engineers the ability to obtain a mathematical model representation of an aircraft from flight data. Use of this information can have various applications, such as expanding an aircraft flight envelope, validation of wind tunnel experiments, or enhancing flight simulation for use with pilot training.

Over the past 50 years there have been various techniques developed to extract estimates of aerodynamic coefficients from dynamic maneuvers. This section will give a brief overview of several techniques developed for off-line parameter estimation. In the late 1940's, a frequency response method gained much popularity for use within aircraft analysis. The output from this technique was a frequency response of the vehicle, instead of the coefficients pertaining to the differential equations describing the system. Although this method was popular, the estimates from this method proved to be poor and biased due to the presence of measurement noise in the data. Another procedure, known as analog matching, pertained to collecting flight data and the programming of an analog computer with a model of the test aircraft under investigation. The measured flight data was then overlaid with computed responses on an oscilloscope. Effectiveness of analog matching was found to be limited because the technique depended upon the user and relied upon knowledge of wind-tunnel data ${ }^{1}$. Taylor ${ }^{1}$ explained about the necessity of automating this fitting process to improve the efficiency of the technique. In 1990, Balderson ${ }^{2}$ implemented such an automated version called digital matching for a Cessna U-206 aircraft at West Virginia University.

In 1966 the Dryden Flight Research Facility, currently known as DFRC, began developing a digital method, known as the ML method ${ }^{3}$, for extracting aircraft derivatives. Use of this technique has helped with the development of various aircraft programs such as the X-15, F-8C, F-111A, F/A-18, X-31, and SR-71 to name a few. During the 1970's the estimation program was found to been effective in analyzing about $89 \%$ of the aircraft stability and control maneuvers attempted ${ }^{3}$. In 1972, a study 
conducted by Iliff and Taylor $^{1}$ modified the NR minimization technique for determining stability derivatives and compared this technique with analog matching and least squares schemes. Older least square approaches gave poor response fits because the computed responses were not a factor in the minimization step. A modified version of this technique included the use of state vectors within the error minimization. This least squares procedure involved minimizing an integral square of the state equation error. By obtaining measured values for the states and derivatives, a cost functional could be minimized. Iliff and Taylor found that a NR technique was necessary to yield reliable results. The cost functional revealed the difference between the computed responses based upon estimated derivatives and the measured flight data responses.

In 1978, Iliff ${ }^{4}$ described several techniques used for estimating coefficients from dynamic flight data, including a straightforward computation of the parameters to solve for unknown aircraft derivatives. Steps for obtaining reasonable results had evolved into producing complex calculations that required extensive computer resources to complete an analysis. In 1987, Iliff $^{5}$ presented results using the ML method, showing the capabilities of increased computer resources for studies of the F-14 and space shuttle programs.

In 1988, a study ${ }^{6}$ was performed with the X-29A demonstrator using ML. The parameters analyzed were used in correlating aerodynamic effects with flight control system stability margins. The model used a combination of wind tunnel and computer analysis results, including wind tunnel work with a 1/8 scale model of the actual aircraft, simulating flight conditions at an altitude of 30,000 feet and Mach 0.9.

In the later half of the 1990's, researchers at West Virginia University, using data from the F/A-18 High Alpha Research Vehicle (HARV) program, performed several PID investigations for analysis of aerodynamic parameters. One study ${ }^{7}$ investigated the nonlinearities and coupling effects associated with both longitudinal and lateral directional dynamics at high angles of attack. A technique developed by Kalviste ${ }^{8,9}$ was applied for modeling the cross-coupling effects due to high alpha flight conditions. Another study by Paris $^{10}$ developed a complete model of the HARV dynamics at high alpha regimes using ML and comparing the results with a Neural Network (NN) PID based algorithm. 
As a result, it was found that the ML technique tends to yield the most reliable results for extracting stability derivatives from flight test data. Over the years, many applications of the ML technique have been performed and show the reliability of the technique. A ML method using a NR minimization technique was implemented and used for this research effort.

\subsection{RPV / UAV Research}

Over the last decade, there has been an increase in demand for use of Unmanned Aerial Vehicle (UAV), also known as Remotely Piloted Vehicles (RPVs) within commercial and military research activities. Some aspects of military research have included flight envelope expansion, survey missions, and the testing of unconventional aircraft configurations. While commercial research areas have included a focus in the areas of testing fault tolerant flight control systems (FTFCS) and aircraft technology. In particular, the DRFC has had substantial involvement with UAVs ${ }^{11}$. Examples of UAVs programs include the X-36 and Pathfinder, which provide scientists and engineers with a low-cost and low-risk means of testing new concepts. Aerodynamic derivatives are constantly under investigation for use with aircraft simulations, updating aerodynamic wind-tunnel estimates and expanding flight testing envelopes for research aircraft. These small aircraft vehicles can allow for new concepts to be test flown without risk to pilot and personnel.

Currently universities are becoming more active in the developing and testing of UAV and RPV aircraft for both commercial and military applications. During 1998-99, NC State University Aerospace Department ${ }^{12}$ worked with the Unmanned Aerial Vehicle (UAV) Flight Research Group. This project used a 17.5\% scaled F/A-18 E instrumented RPV to collect dynamic flight data for post-flight analysis. Another project at Sydney University ${ }^{13}$ has current research involving both UAV and RPV technology with research focused towards fully autonomous vehicles. This type of research foresees the development of self-piloted vehicles using some form of trajectory planning with autonomous navigation techniques. Other areas of investigation for autonomous vehicles have included system identification, various flight control systems, airframe design, fabrication, and instrumentation ${ }^{13}$. 
RPV vehicles do not require a cockpit for a pilot, which leads designers to create new maneuverable aerodynamic configurations, such as tailless flight vehicles or enhanced aircraft capable of flying at higher angles of attack due to the addition of thrust vectoring. Due to advances in composite materials, small electronics, and the enhanced computer controlled radio equipment, the construction costs of these small test vehicles have been lowered dramatically. This allows researchers to have a low cost solution for testing new concepts instead of using the large and expensive full-scale aircraft counterparts.

\subsection{Online PID Techniques}

In-flight, on-line system identification has become an important topic in the past few years, especially within the general area of FTFCS ${ }^{14,15,16}$. Over the past few decades; off-line techniques have been the main source of aerodynamic modeling information. Normally, aircraft PID analysis was performed off-line using previously recorded flight data. Several statistical methods have been used for PID purposes with the ML method being one of the most widely used approaches ${ }^{1,3,17}$. Input test maneuvers are planned out in advance and implemented within a flight test program. Flight engineers have to schedule test maneuvers, obtain approval to place maneuvers into a flight schedule, execute flight tests, and then evaluate results in a post-flight analysis. With the increase in computing resources it is now possible to obtain modeling information during actual flight testing activities. In recent years, different PID techniques have been proposed for on-line real-time applications to be implemented on-board aircraft ${ }^{14,15}$. The resulting adaptive system can then be applied and modified during an actual flight testing. In particular, the on-line extension of the PID process has immediate and potentially very important applications for control of time varying aircraft systems, such as an aircraft subjected to substantial changes in dynamic and aerodynamic characteristics. For control applications a fast convergence of the parameters to be estimated can be a point for this type of application.

There are several methods involving both a frequency and time domain based PID techniques. On-line time domain PID techniques mainly include variations of the LS regression method, such as Recursive Least Square (RLS) ${ }^{18,19}$, RLS with a forgetting 
factor $^{20}$, a Modified Sequential Least Square (MSLS) ${ }^{21}$, a real-time Batch Least Squares $(\mathrm{BLS})^{22,23}$, and Extended Kalman Filtering $(\mathrm{EKF})^{24}$. Within the frequency domain, the technique relies on a discrete Fourier Transform. Real time applications, of any of these methods present a challenge due to a possible lack of information for PID purposes within the flight data, such as the potential for unavailable independent control inputs used for PID purposes, due to a possible prolonged steady state flight condition. 


\section{Chapter 3}

\section{Development and Construction of the B777 Aircraft Model}

\subsection{Development of the B777 Aircraft Model}

The design and construction of the WVU Boeing 777 model aircraft was the result of a collaboration of MAE researchers and Craig Aviation. The overall grant objective for creating and flying the RPV projects was to produce a viable flying test bed for future applications of Neural Network (NN) technologies and the developing of fault tolerant flight control systems (FTFCS), like Sensor Failure Detection, Identification, and Accommodation (SFDIA) schemes. The purpose of this research work was to obtain derivatives of the B777 to be used for future flight control applications. For the purpose of this research effort, a series of flight tests were necessary to obtain the estimates of the model. Initially, two main design issues had to be addressed for the aircraft models. One was that the RPV had to be designed to carry a twelve-pound payload of additional aircraft instrumentation, and second, have a flight time capable of collecting several aircraft maneuvers in one flight. The target maneuver flight time was selected to be approximately six to seven minutes, with a safety factor of extra fuel onboard. Earlier work included the creation of a "sister" prototype model, the Boeing 747 (shown in Figure 3.1.1). A photo of the B777 test bed is shown in Figure 3.1.2.

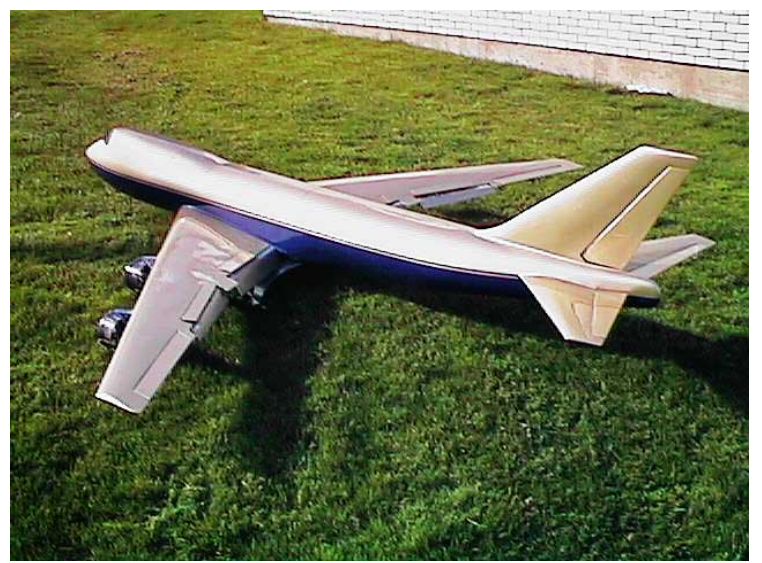

Figure 3.1.1 WVU B747 Model

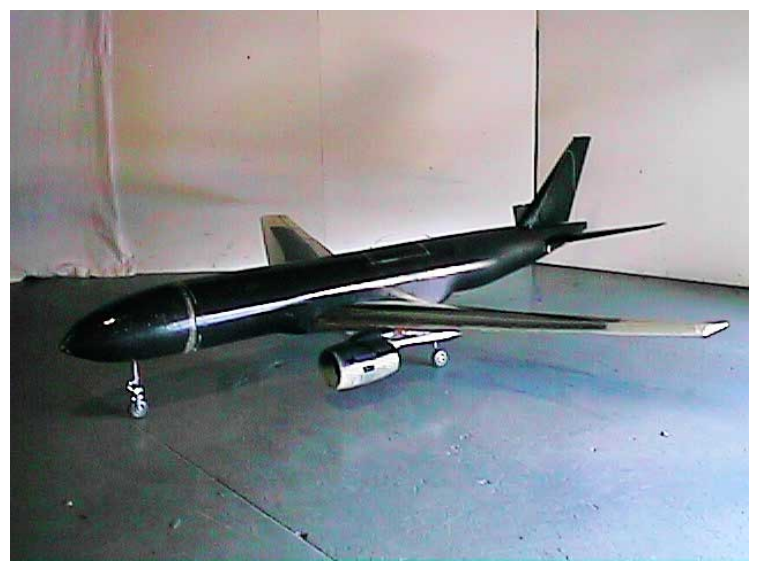

Figure 3.1.2 WVU B777 Model

The development of the WVU B747 aircraft allowed for issues related to construction, propulsion, and instrumentation to be addressed for initial flight testing activities. The B747 aircraft was comprised of a three-component system, a main fuselage body, along 
with the left and right wing attachments. It was found that this three-component system created difficulties with propulsion and fuel delivery systems. Also, the additional structural weight for attaching the left and right wing sections exceeded the weight requirements for the aircraft. The B747 aircraft was found unable to meet the required design specifications for both the payload and flight time requirements. For this reason, the B747 test bed was not used for actual flight tests, but allowed for testing of the first generation instrumentation package. In fact, the model was originally designed and manufactured as a "scale" model aircraft, intended for sale to the general public. The instrumentation package tests were achieved using a ground based vehicle-testing frame. Figure 3.1.3, shows a sketch of a vehicle test frame that was used for ground testing of the B747.
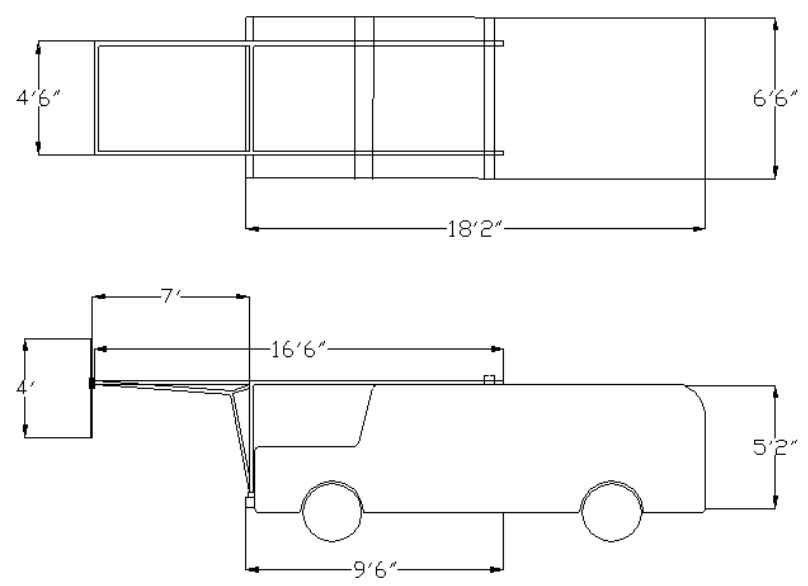

Figure 3.1.3 Sketch of ground based vehicle test frame

Using a Chevrolet Suburban vehicle, the B747 was mounted and suspended in front of the vehicle via a ball joint apparatus and a linear steel shaft connected to the truck test frame. The ball joint, located at the aircraft center of gravity, allowed the aircraft to move in pitch, roll, and yaw, along with z-direction motion from the linear steel shaft. This device provided an environment for evaluating the instrumentation capabilities without endangering the aircraft model or any of the electronic components. The frame testing apparatus provided an opportunity for the pilot to simulate maneuvers and gain experience, while evaluating and testing instrumentation payload. 
Learning from the drawbacks of the B747 design, the B777 aircraft was based upon a more conventional two-component approach, a main fuselage section and a onepiece wing assembly. Both the wing and fuselage sections were designed around the aircraft instrumentation requirements. Another major design change involved the main propulsion system. The B747 had four small engines, which required heavy maintenance time and had difficulties with balancing engine thrust performance. For that reason, two larger engines were selected for increased thrust and ease of fuel delivery for the final wing assembly.

\subsection{B777 Aircraft Model}

The flight testing aircraft team included professors, graduate research assistants, and undergraduate students from the Mechanical and Aerospace Engineering department, with a subcontractor, Craig Aviation, to produce the airframe. Figure 3.2.1 is a photo of the research flight testing crew.

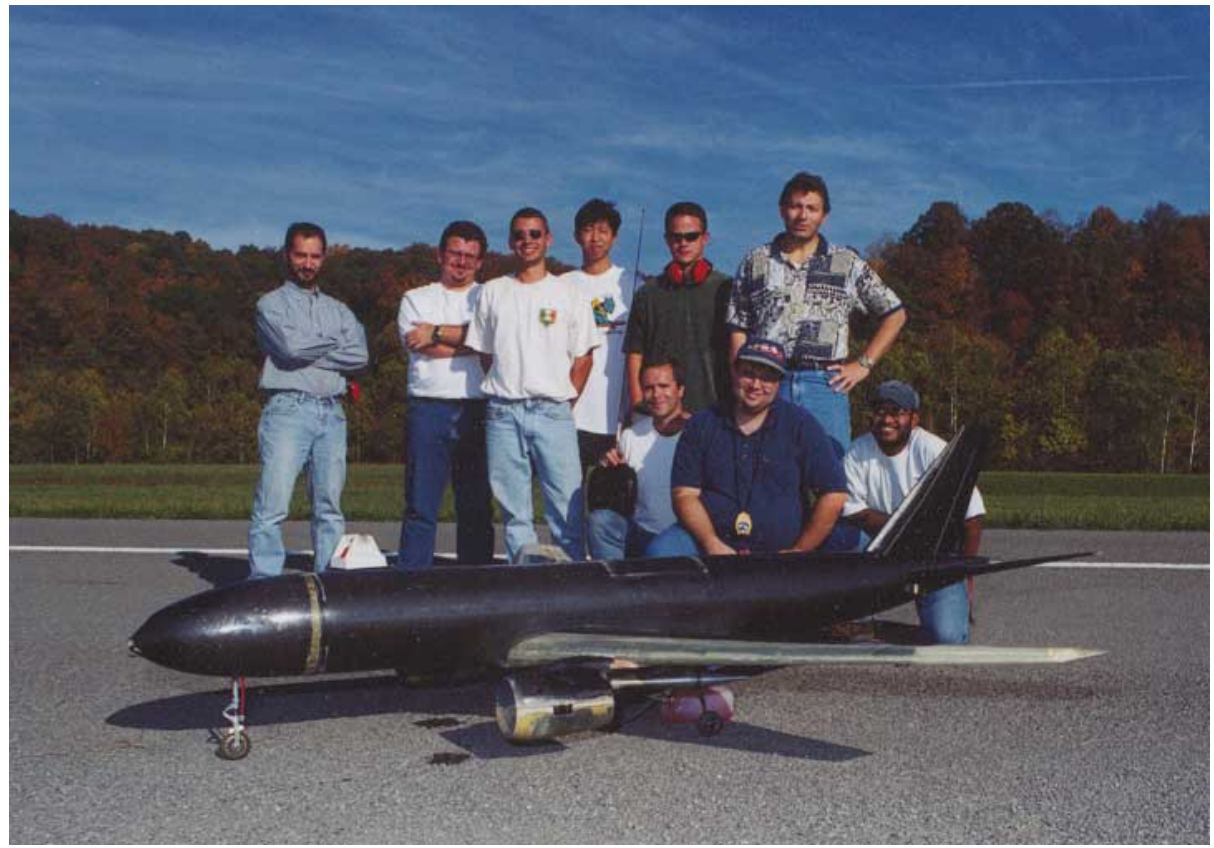

Figure 3.2.1 Aircraft construction and flight testing team

left to right (bottom row): John Craig, Brad Seanor, Srikanth Gururajan; (top row): Diego Del Gobbo, Francesco Nasuti, Peter Cooke, Yu Gu, Ben Reid, Dr. Marcello Napolitano (Professor) 
The aircraft design and fabrication began from aircraft drawings supplied by Boeing. Since the model was a research aircraft, it was not necessary to build a perfectly scaled model, which was normally sold to the general public. In fact, the wings were modified, increasing the wing area, providing a larger tip ratio and a lower sweep angle for improved aerodynamic efficiency at low speeds.

There are two major structural components that make up the B777: (i) fuselage, and a (ii) one-piece wing section. The wing section included the addition of a left and right engine nacelle. An original plug was created for each of these separate components, and then used to create the fiberglass molds. The production of the plug had to be precise at the various connection points to avoid potential structural failures. Figures 3.2.2 through 3.2.4 show the structural parts created from each of the sub-contractor's molds.

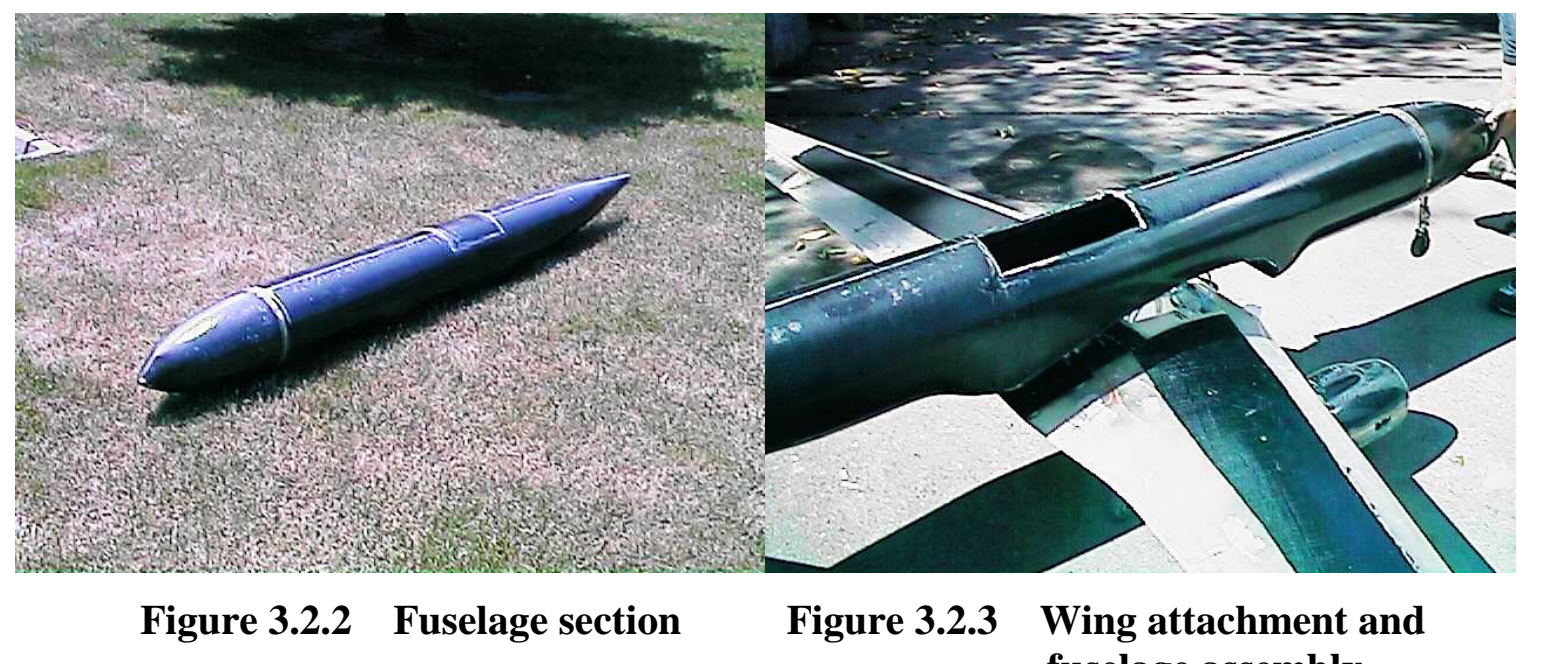
fuselage assembly

The complete aircraft was manufactured from various materials, including a high volume of fiberglass, carbon fiber, foam, and lightweight modeling plywood. The fuselage made extensive use of carbon fiber material that provided the necessary structural strength, and thus eliminated the need for a large number of bulkheads, in turn providing for increased payload capacity. Figure 3.2.4 shows a photo of the separate left and right engine nacelles along with one of the engine and ducted fan assemblies. 


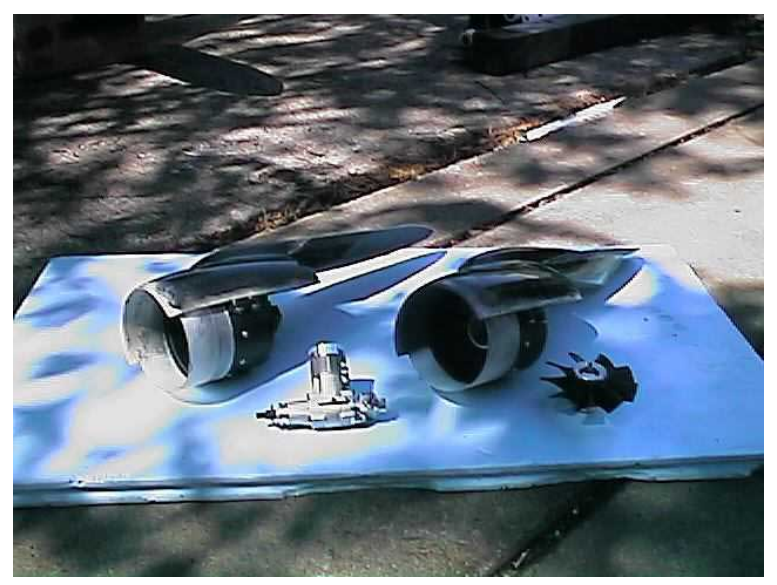

Figure 3.2.4 Engine nacelles and fan assembly

The fuselage structure was built with three access panels, namely the main fuselage hatch, rear fuselage hatch and nose cone assembly that allowed for the following equipment to be installed:

- main fuselage hatch provided access to:

-- main instrumentation panel

-- $\quad$ signal conditioning interface

-- $\quad$ gyro/accelerometer sensor unit

-- $\quad$ fuselage/wing interface

-- main instrumentation battery

- rear fuselage hatch provided access to:

-- $\quad$ tail section servos

-- $\quad$ potentiometers for tail control surfaces

-- $\quad \mathrm{R} / \mathrm{C}$ System receiver

- nose cone assembly provided access to:

-- $\quad$ air-probe and pressure sensors

-- $\quad$ angle of attack and sideslip flow vanes

-- main computer system

-- nose section servos for front landing gear

Shown in Figure 3.2.5, the one-piece wing structure design provided easy access to all wing controls and fuel storage. 


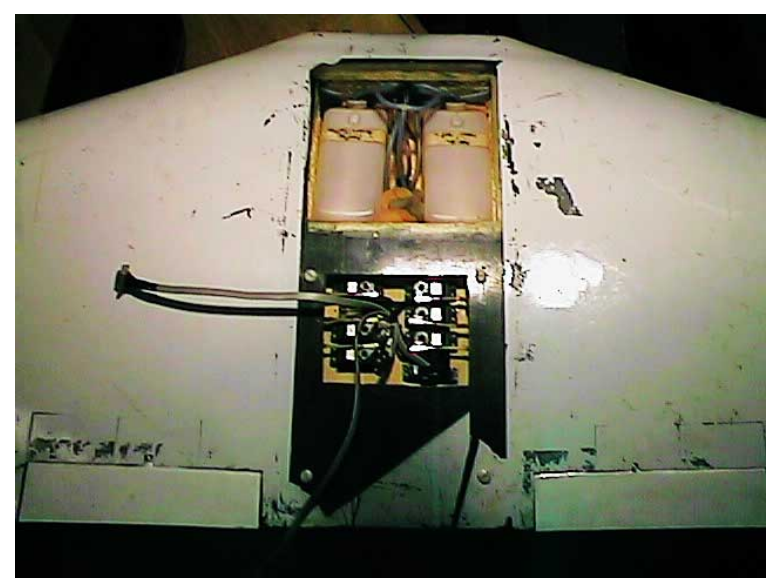

Figure 3.2.5 Wing servo system and fuel bay

Initially the wing control surfaces only included the left and right ailerons and two throttle servos for the preliminary R/C testing. For initial testing, our pilot found the need to reduce the aircraft's speed during the approach and landing phases. After several initial flights, both an inboard and outboard flap system was added to the aircraft. Table 3.2.1 shows the mass and geometric characteristics for the B777 model.

Table 3.2.1 - Mass and geometric characteristics for the $\mathrm{B} 777$

\begin{tabular}{|l|c|}
\hline length & $8.75 \mathrm{ft}$ \\
\hline $\mathrm{b}$ (Span) & $8.92 \mathrm{ft}$ \\
\hline$\lambda$ (Taper Ratio) & 0.27 \\
\hline $\mathrm{Cr}$ (Root) & $2.00 \mathrm{ft}$ \\
\hline $\mathrm{Ct}$ (Tip) & $0.54 \mathrm{ft}$ \\
\hline$\Lambda_{\mathrm{LE}}$ & $27.0 \mathrm{deg}$ \\
\hline Aspect Ratio & 7.02 \\
\hline $\mathrm{S}$ (Wing Area) & $11.33 \mathrm{ft}^{2}$ \\
\hline Mean Aerodynamic Chord & $1.41 \mathrm{ft}$ \\
\hline Elevator total area & $0.48 \mathrm{ft}^{2}$ \\
\hline Aileron total area & $0.64 \mathrm{ft}^{2}$ \\
\hline Rudder total area & $0.33 \mathrm{ft}^{2}$ \\
\hline Elevator span (left \& right) & $2.64 \mathrm{ft}^{2}$ \\
\hline Aileron span (left \& right) & $2.67 \mathrm{ft}^{2}$ \\
\hline Rudder span (left \& right) & $1.46 \mathrm{ft}^{2}$ \\
\hline
\end{tabular}


Table 3.2.2 shows the inertia data values for the B777 model.

Table 3.2.2 - Experimentally determined inertia data for the B777

\begin{tabular}{|c|c|}
\hline $\mathrm{I}_{\mathrm{xx}}$ & 5.20 slug- $\mathrm{ft}^{2}$ \\
\hline $\mathrm{I}_{\mathrm{yy}}$ & 6.34 slug- $\mathrm{ft}^{2}$ \\
\hline $\mathrm{I}_{\mathrm{zz}}$ & 6.97 slug- $\mathrm{ft}^{2}$ \\
\hline $\mathrm{I}_{\mathrm{xz}}$ & 0.28 slug- $\mathrm{ft}^{2}$ \\
\hline $\mathrm{m}$ & 1.45 slugs \\
\hline
\end{tabular}

Figure 3.2.6 below shows a layout configuration of the molded aircraft parts.

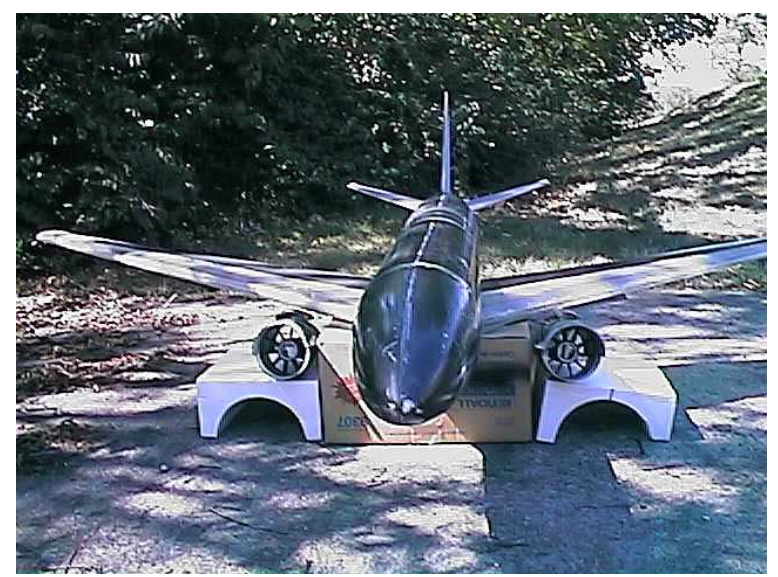

Figure 3.2.6 Initial layout of molded parts

Initial performance values of the aircraft for the R/C-only flights and are listed in Table 3.2.3 below.

Table 3.2.3 -R/C only flight parameters

\begin{tabular}{|c|c|c|}
\hline Weight and $48 \mathrm{oz}$ fuel & 30.2 & $\mathrm{lb}$. \\
\hline S (wing area) & 11.3 & $\mathrm{ft}^{2}$ \\
\hline Static thrust & 24 & $\mathrm{lb}$. \\
\hline Thrust/Weight Ratio & 0.79 & -- \\
\hline Wing Loading & 43 & $\mathrm{oz} / \mathrm{ft}^{2}$ \\
\hline $\mathrm{V}_{\text {stall }}$ & 50 & $\mathrm{ft} / \mathrm{sec}$ \\
\hline $\mathrm{V}_{\text {takeoff }}$ & 60 & $\mathrm{ft} / \mathrm{sec}$ \\
\hline
\end{tabular}




\subsection{Radio Control (R/C) System}

The radio control $(\mathrm{R} / \mathrm{C})$ system was based on a 10-channel programmable menu driven radio system. The Airtronics Infinity 1000A transmitter with a 16-bit microprocessor, shown in Figure 3.3.1 below, was chosen for the capability of customizing aircraft controls.

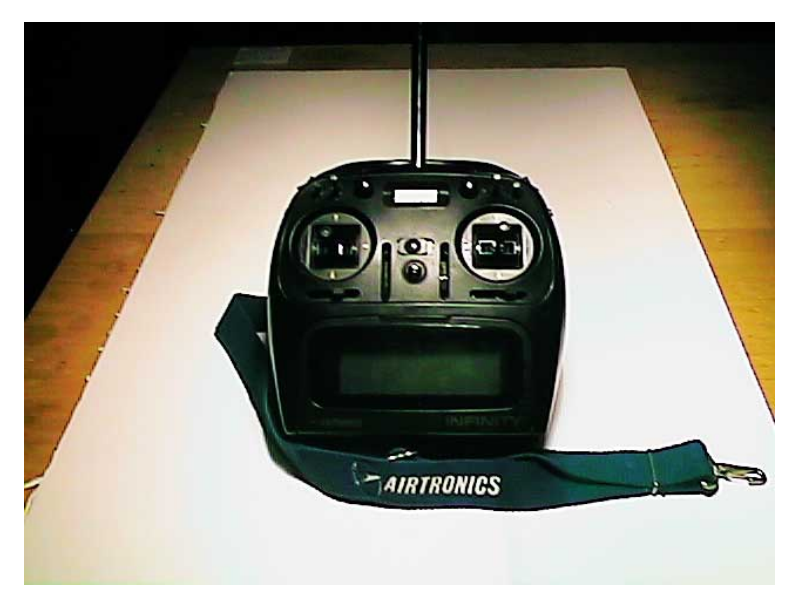

Figure 3.3.1 Airtronics Infinity 1000A 10-channel transmitter

The unit was equipped with a non-volatile memory, 1024-bit high resolution Pulse Code Modulation (PCM) and a LCD display panel. A list of features included programmable flight modes, programmable soft switches, timer, tachometer, and 8 programmable model memories. Nine pre-assigned mixers and eight definable mixers were available on the transmitter to allow the pilot to customize control inputs. An example would be when the flaps are deployed; an automatic elevator input can be given to provide a stable pitch motion without additional pilot intervention. The optional synthesized RF module and receiver allowed the pilot to select from 50 available aircraft frequencies at the touch of a button. For recreational R/C radio systems there are three radio modulations available:

- $\underline{A M}$ - Amplitude Modulation, which transmits by a variation in the amplitude of signals, it is subject to interference more than FM;

- $\underline{F M}$ - Frequency Modulation, which transmits signals by variations in frequency, reduces the risk of "glitches" due to signal interference;

- $\underline{P C M}$ - Pulse Code Modulation uses a binary code to digitize the signal, providing the most accurate signal possible. 
For improved performance and minimal radio interference, a PCM radio was chosen for use with this project. Another important radio feature was the dual rate switches. A dual rate switch can reduce or increase the amount of servo travel making the control less or more sensitive. By selecting a low rate, an over-responsive aircraft can be made easier to control. All three major control channels, elevator, aileron, and rudder had this dual rate feature available to the pilot. The transmitter is powered by a 9.6 volt 1100 mAh NiCd battery and three 4.8 volt 1200 mAh NiMH batteries powered the onboard receiver and servos. This battery capacity provided a reliable power source for all $\mathrm{R} / \mathrm{C}$ systems and allowed for several flight tests without the need of recharging. Figure 3.3.2 shows a layout of the $\mathrm{R} / \mathrm{C}$ servo setup.

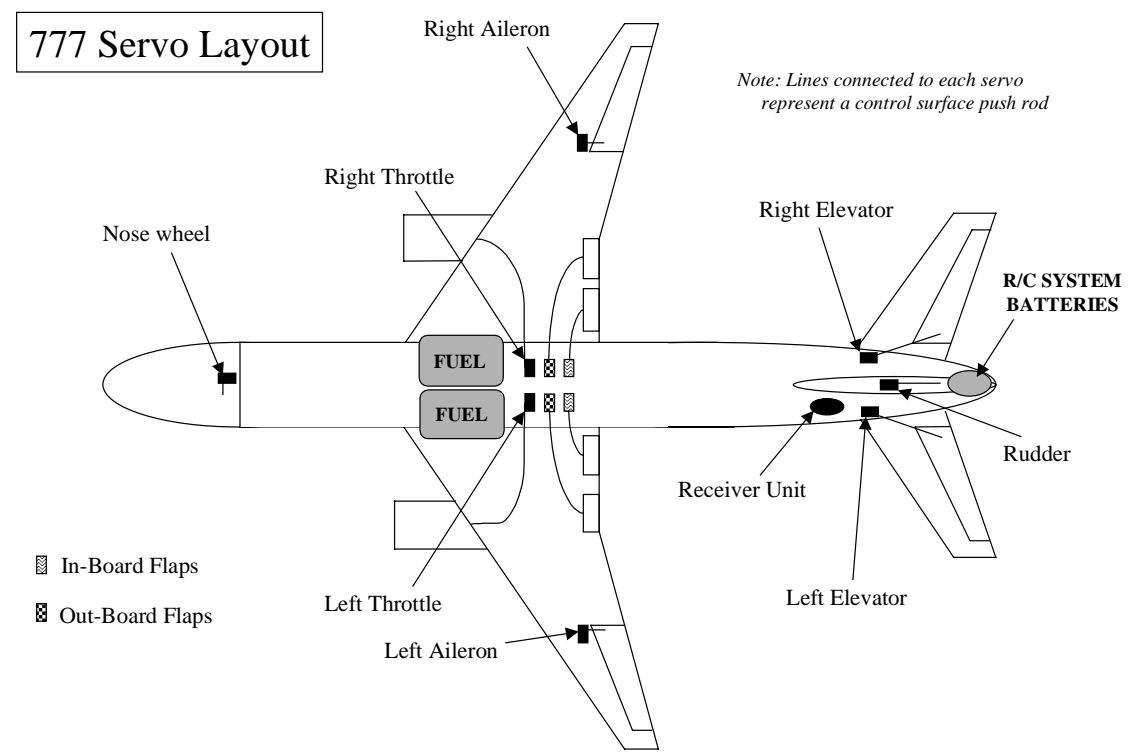

Figure 3.3.2 Servo layout for the B777 model 
Table 3.3.1 lists the specific Airtronics servos used on the B777, denoted by their corresponding position within the aircraft (rudder, nose gear, etc.).

Table 3.3.1 - Current listing of Airtronics servos used for the B777 model

\begin{tabular}{|c|c|}
\hline Servo Position & Model number \\
\hline Rudder & 94738 \\
\hline Left and Right Elevator & 94161 \\
\hline In-Board and Out-Board Flaps & 94102 \\
\hline Left and Right Aileron & 94738 \\
\hline Nose Gear & 94102 \\
\hline Left and Right Engine Throttles & 94102 \\
\hline
\end{tabular}

Table 3.3.2, provides specifications of the torque and transit time (to rotate 60 degrees) values for each servo model. Special emphasis was placed on selecting servos for each of the main aircraft control surfaces. For important controls, such as elevator, rudder, and ailerons, high torque value and faster response times were required in order to provide the pilot with improved handling characteristics. Servos controlling the engine throttles, flaps, and nose gear were not required to have high torque and fast response characteristics. Actual forces applied to these secondary controls are minimal compared to the forces experienced on the elevator, rudder and aileron control surfaces.

Table 3.3.2 - Specifications for various aircraft servos

\begin{tabular}{|c|c|c|c|c|c|c|}
\hline $\begin{array}{l}\text { Model } \\
\#\end{array}$ & $\begin{array}{l}\text { Dimension } \\
(\mathbf{L} \times \mathbf{W} \times \mathbf{H})\end{array}$ & $\begin{array}{l}\text { Wt. } \\
\text { Oz. }\end{array}$ & $\begin{array}{l}\text { Torque } \\
\text { Oz., } 4.8 \mathrm{~V}\end{array}$ & $\begin{array}{l}\text { Transit } \\
\text { Time } \\
60 \text { degrees }\end{array}$ & $\begin{array}{l}\text { Motor } \\
\text { Type }\end{array}$ & Bearings \\
\hline 94102 & $1.54 \times 0.079 \times 1.42$ & 1.59 & 50 & 0.22 & Std 3-pole & No \\
\hline 94141 & $1.42 \times 0.6 \times 1.29$ & 1.17 & 45 & 0.20 & Coreless & Single \\
\hline 94161 & $1.54 \times 0.79 \times 1.65$ & 2.5 & 135 & 0.25 & Std 3-pole & Double \\
\hline 94738 & $1.54 \times 0.79 \times 1.38$ & 1.95 & 71 & 0.21 & Coreless & Double \\
\hline
\end{tabular}




\subsection{Propulsion System}

The B777 propulsion system featured two O.S.91 VR-DF ducted fan engines, shown in Figure 3.4.1, each provided approximately 12 pounds of static thrust for a total of 24 pounds of thrust. As stated earlier, each engine nacelle was molded and attached to the one-piece wing section.

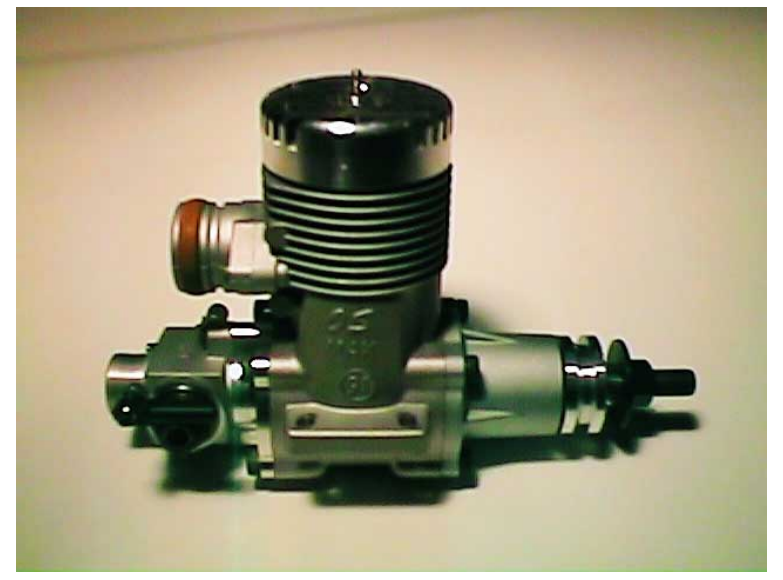

Figure 3.4.1 O.S.91 VF-DR (small head) ducted fan engine

Specifications from the engine manufacturer are listed in Table 3.4.1:

Table 3.4.1 - Engine specifications for O.S. 91 VF-DR ducted fan engine

\begin{tabular}{|lc|l|l|l|l|}
\hline $\begin{array}{l}\text { Displacement } \\
\text { in) }\end{array}$ & (cu & $\begin{array}{l}\text { Bore } \\
\text { (inch) }\end{array}$ & $\begin{array}{l}\text { Stroke } \\
\text { (inch) }\end{array}$ & RPM & Weight (oz) \\
\hline 0.900 & 1.091 & 0.965 & $2,500-25,000$ & 23.37 \\
\hline
\end{tabular}

The operating RPM range for this engine was approximately $7000 \mathrm{rpm}$ at idle speed setting to a maximum value of 19,000 RPM on the ground. While in flight, the engine will unload and run at a higher rpm values, noting the 25,000-RPM maximum value in Table 3.4.1. A tune-pipe exhaust was employed to help yield a maximum engine performance.

The O.S.91 engine used a simple ignition system, a glow plug rather than a spark plug. A battery-operated glow starter, heats the glow plug while the engines are turned over using a $12 \mathrm{~V}$ electric starter. Figure 3.4.2 displays the starter panel system along with the electric starter and glow plug connector for the ducted fan engines. 


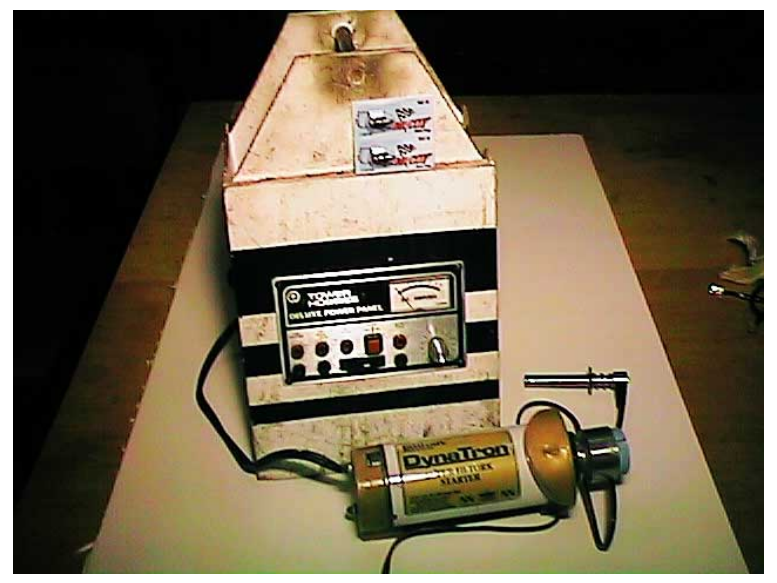

\section{Figure 3.4.2 Field box starting equipment for propulsion system}

A "Ramtec" ducted fan unit, purchased from AeroLoft Designs, was used with the engines. This fan unit contained a nine-rotor blade system with an engine shroud/rotor hub assembly, shown in Figure 3.4.3. The nacelle-mounting bracket was constructed from a handcrafted plug/mold to resemble an actual engine mount on the real Boeing 777 aircraft.

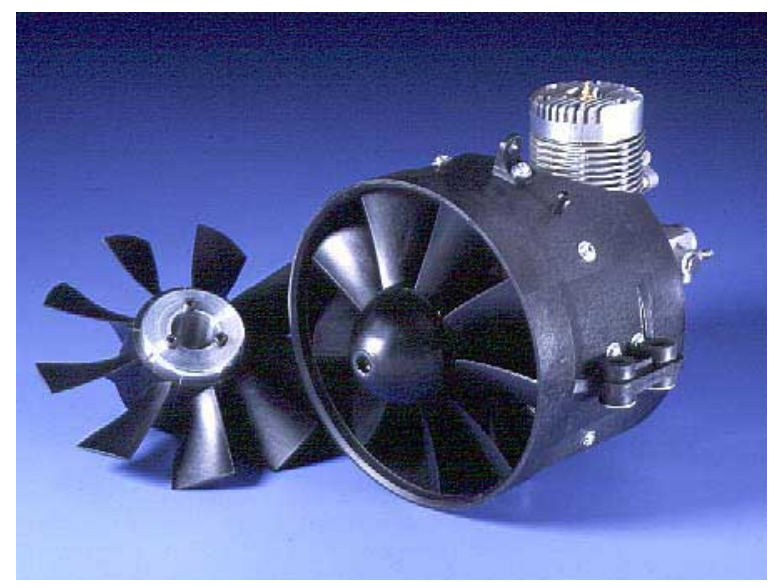

Figure 3.4.3 Ramtec ducted fan unit (AeroLoft) with engine in background

The fuel system was comprised of two pressurized 24-oz. tanks, which allowed for a nine-minute maximum run time at full throttle. The fuel used was a Nitro methane mixture containing $5 \%$ to $15 \%$ nitro with $15 \%$ to $20 \%$ oil content for lubrication. A maximum flight time of approximately seven minutes was regularly scheduled for each flight test. This reserve fuel supply provided the safety factor necessary to allow for emergency situations, such as adverse weather conditions causing a need for multiple landing approaches. An additional ground fuel supply was used at the start of all flight tests, allowing for the main tanks to remain full before launch. 


\subsection{Instrumentation System}

The on-board computer instrumentation system included an "All-In-One" CPU board, RAM Disk card, and data acquisition card mounted onboard a passive backplane. Figure 3.5.1 shows a block diagram of the instrumentation components.

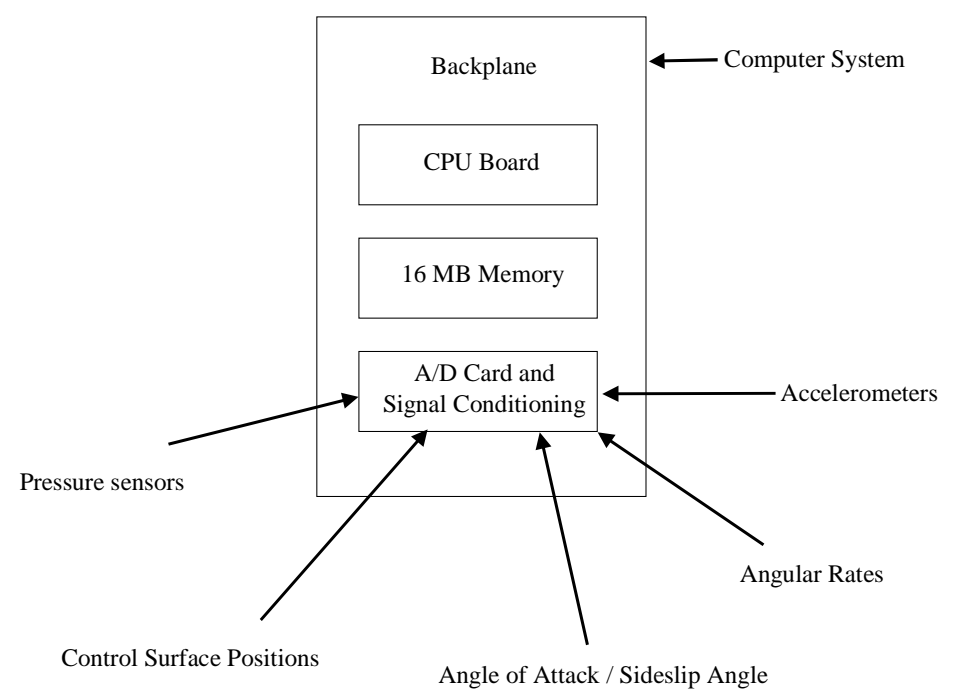

\section{Figure 3.5.1 Schematic of the instrumentation layout}

The package sensors provide measurements for obtaining the following aircraft parameters:

- control surface positions $\left(\delta_{\mathrm{e}}, \delta_{\mathrm{a}}, \delta_{\mathrm{r}}\right)$

- accelerations $\left(A_{n}, A_{x}, A_{y}\right)$

- angular rates (p, q, r)

- angle of attack and sideslip angle $(\alpha, \beta)$

- $\quad$ airspeed (V) and altitude

Prior to each flight, the control surfaces and flow angle vanes were calibrated at the airfield facility. The calibration was then applied to the recorded flight data to convert all parameters to the proper engineering units for post flight analysis. The real-time data acquisition software was stored onboard a flash RAM disk along with all recorded sensor information. During each test flight, the real-time data acquisition program stored a data file with sensor voltages. After landing, the data file was then transferred via a serial port connection to a laptop for post flight analysis. 


\section{Computer System}

The computer system, purchased from Advantech, was a Pentium Pro (model PCA-6167) CPU Card with an ISA/PCI interface (Figure 3.5.2) and Intel Pentium Pro $200 \mathrm{MHz}$ CPU with $32 \mathrm{MB}$ of RAM on the card.

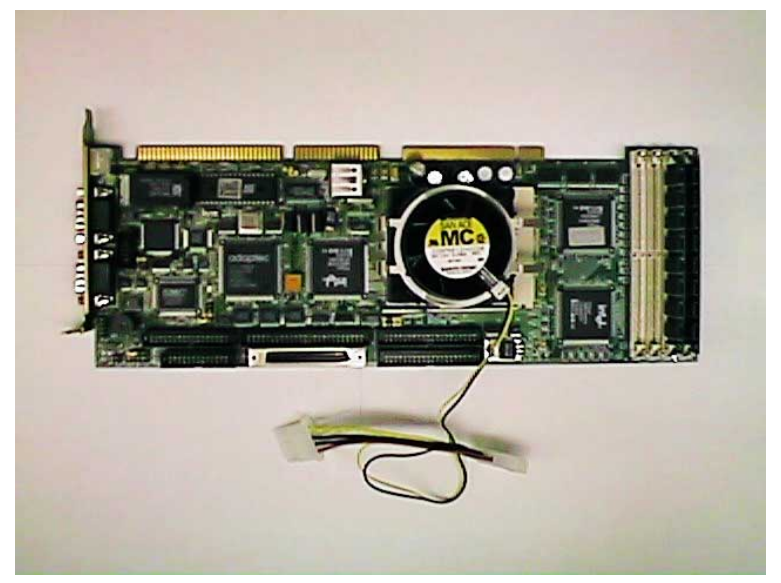

\section{Figure 3.5.2 Pentium Pro all-in-one CPU Card (Advantech)}

All computer cards are connected with a passive backplane (Figure 3.5.3) which contains three PCI slots, four ISA slots, of which two are available for use with CPU slots. One of the CPU slots holds the all-in-one computer card; one ISA slot houses the data acquisition card. The flash ram and video card then used the remaining two ISA slots available. Figure 3.5.3 shows the layout of the passive backplane. The video card was only necessary for ground operations and was removed prior to every test flight.

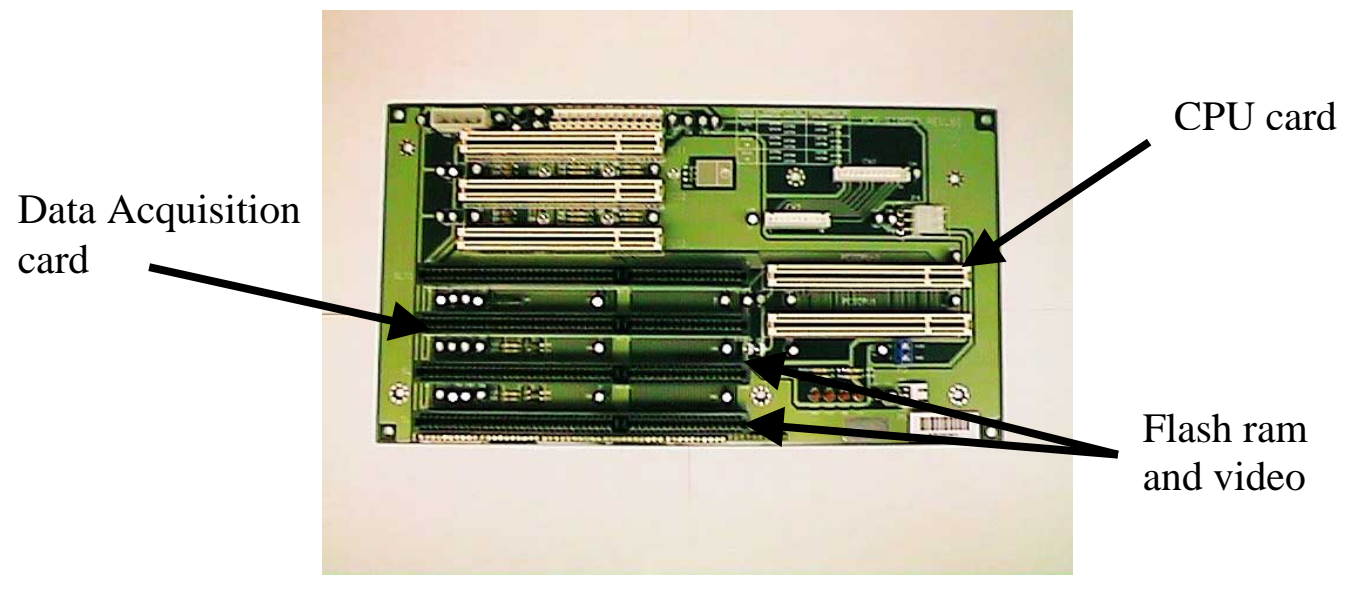

Figure 3.5.3 Passive backplane

A flash card storage system was chosen over a hard disk based solution due to possible problems induced from vibration. The PCD-897 DiskOnChip® 2000 Flash PC 16 MB card (Figure 3.5.4) shows a solid-state disk in a standard 32-pin DIP package. It is a fast 
and economical Flash disk used for portable applications with limited space. Other advantages of the single-chip flash memory card included a reduction in weight and size and lower power consumption when compared with a hard disk storage system.

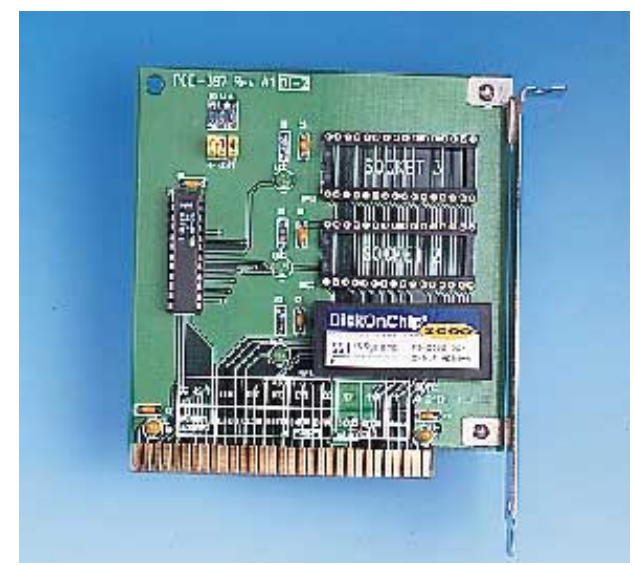

Figure 3.5.4 16-MB flash ram card

The data acquisition card (shown in Figure 3.5.5) was connected via a 50-pin ribbon cable connector to a signal-conditioning interface unit. The unit featured 48 (single ended) channels with 12-bit analog inputs. This large number of channels could allow for future expansion, if necessary.

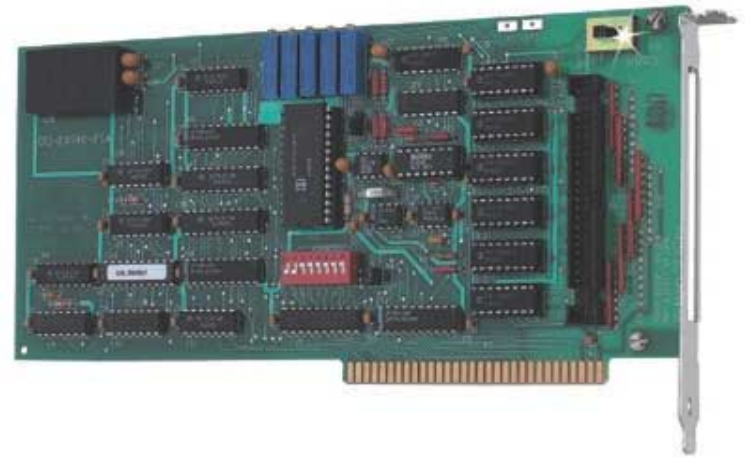

\section{Figure 3.5.5 CIO-DAS48-PGL data acquisition card}

The instrumentation package was mounted in a custom aluminum chassis that was designed to endure runway-induced vibration; padded foam was also added to protect the computer equipment. Overall, the RAM Disk memory and the 21.6-volt computer battery were capable of storing 11 minutes of recorded flight data at the selected sampling rate of $100 \mathrm{~Hz}$. Figure 3.5.6a and Figure 3.5.6b show photos of the custom computer chassis from a front and top prospective. 


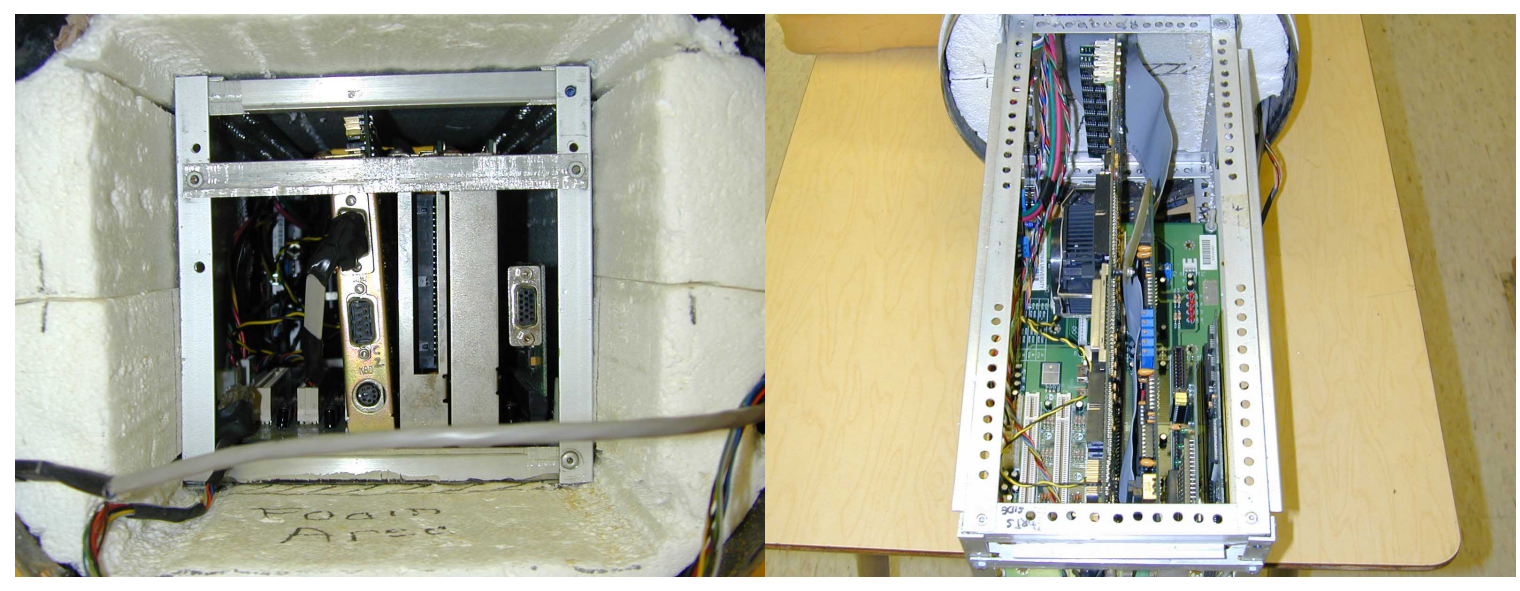

Figure 3.5.6a Custom computer chassis Figure 3.5.6b Custom computer chassis (front view - fuselage)

(top view)

The battery pack is comprised of $18,1.2$ volt NiCd cells with a $2000 \mathrm{mAh}$ capacity. DCto-DC converters were used to obtain the required 5 and 12 volts necessary for computer and sensor equipment. Figure 3.5.7 provides a top view layout of the instrumentation package.

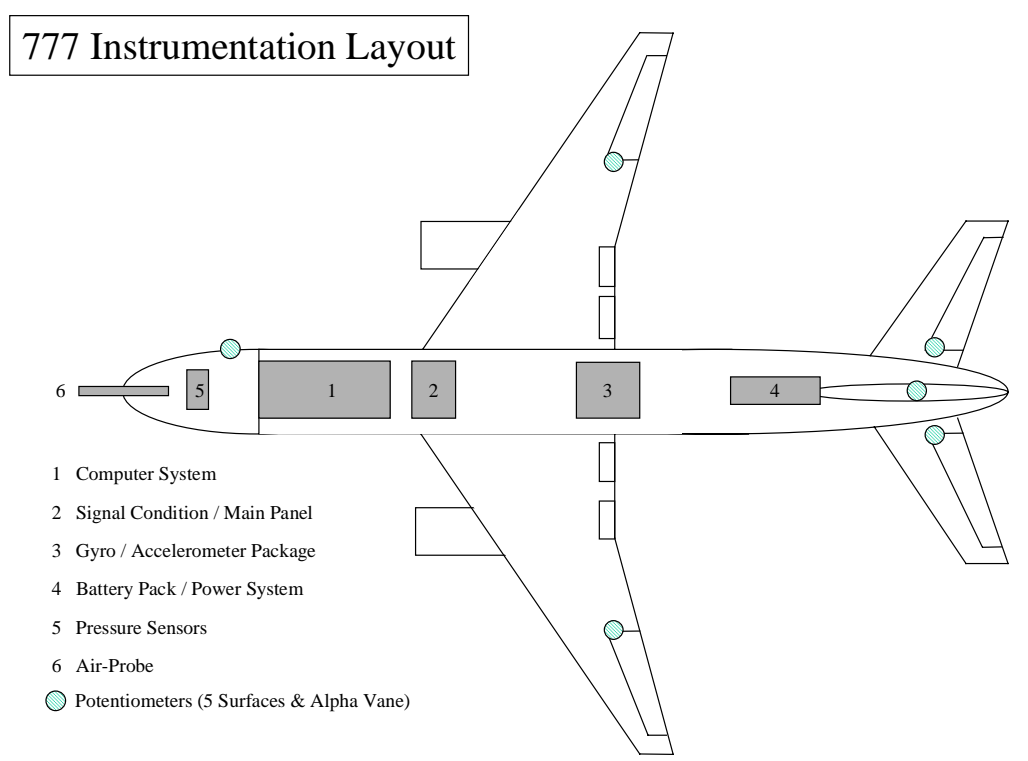

Figure 3.5.7 Top view of the instrumentation layout for the overall aircraft system 
Table 3.5.1 shows the estimated aircraft design parameters for flights with electronic payload. In comparison, the takeoff speed found from test results, using a radar gun, were found to be approximately $60 \mathrm{ft} / \mathrm{sec}$.

Table 3.5.1 - Flight parameters with payload

\begin{tabular}{|c|c|c|}
\hline Weight and $48 \mathrm{oz}$ fuel & 44.7 & $\mathrm{lb}$. \\
\hline S (wing area) & 11.3 & $\mathrm{ft}^{2}$ \\
\hline Static thrust & 24.0 & $\mathrm{lb}$. \\
\hline Thrust/Weight Ratio & 0.54 & -- \\
\hline Aspect Ratio & 7.02 & -- \\
\hline Wing Loading & 63 & $\mathrm{oz} / \mathrm{ft}^{2}$ \\
\hline $\mathrm{V}_{\text {stall }}$ & 61 & $\mathrm{ft} / \mathrm{sec}$ \\
\hline $\mathrm{V}_{\text {takeoff }}$ & 73 & $\mathrm{ft} / \mathrm{sec}$ \\
\hline
\end{tabular}

\section{Signal Conditioning Unit}

A signal conditioning unit was necessary to filter the incoming data from the various aircraft sensors and provide information to the data acquisition card. The signal conditioning interface unit was made up of five separate and compact circuit boards. Each major section of the aircraft (wing, tail, etc.) had a sensor line connected with the main interface box, via RJ45 connectors. Figure 3.5.8 shows the inside view between each RJ45 connection and the data acquisition ribbon cable. Figure 3.5.9 shows the interface connection inside of the aircraft fuselage, along with the control panel used for the downloading flight information.

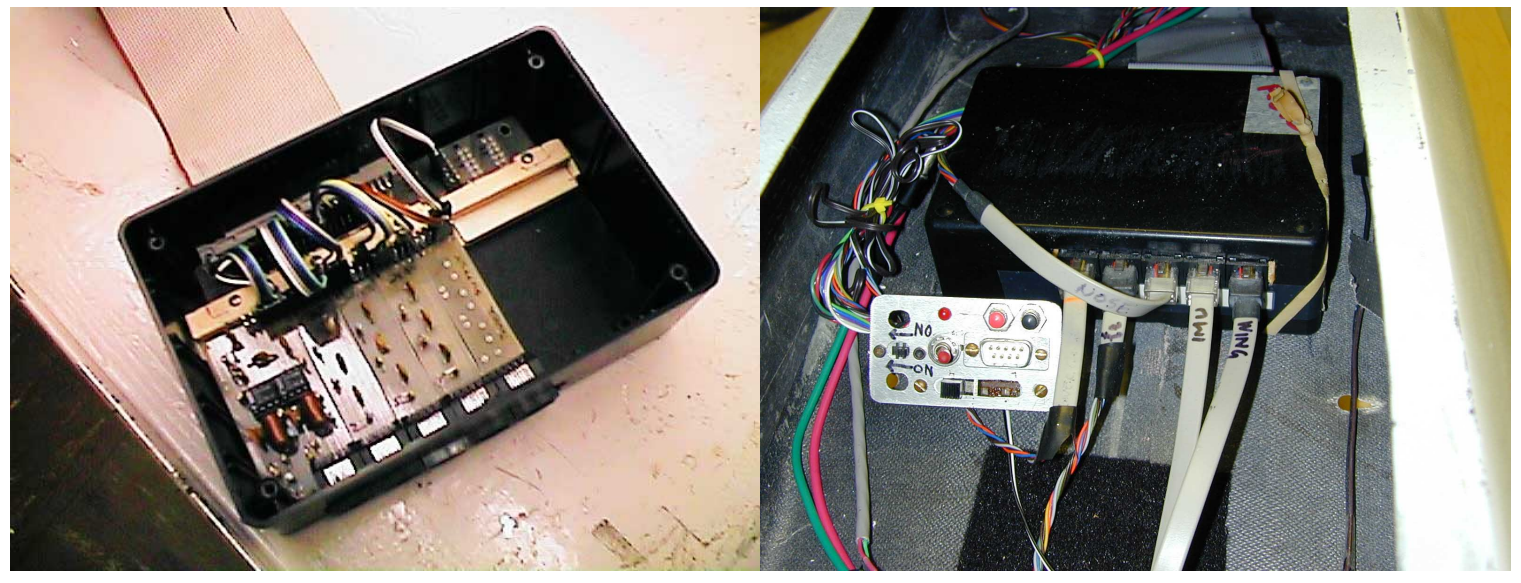

Figure 3.5.8 Signal conditioning interface

Figure 3.5.9 Sensor interface 
This arrangement sectioned off the aircraft sensors into several blocks for ease of maintenance. The various signal blocks included (i) sensors from the wing section, (ii) sensors from the tail section, (iii) gyro/accelerometer sensors, and the (iv) nose section. The fifth board served as a power connection. Power was provided from the computer and fed into the signal conditioner box. The control surface potentiometers, "gyro box" assembly, and nose sensors were powered by tapping into these voltage outlets. With exception of the angular rates, signals from each of these sensor blocks were fed to an onboard $50 \mathrm{~Hz}$ low pass filter to maintain a higher signal to noise ratio. The entire set of signals from the sensors was collected and fed into the data acquisition card through the 50 pin flat ribbon cable.

\section{Angular Rates and Accelerations}

The BEI GyroChip ${ }^{\mathrm{TM}}$ Horizon, shown below in Figure 3.5.10, is a micromachined Angular Rate Sensor (gyro) that uses a one-piece vibrating piezoelectric quartz tuning fork as a sensing element. These Horizon sensors featured a $+12 \mathrm{Vdc}$ Input, 0 to $+5 \mathrm{Vdc}$ output signal with a compact and lightweight design. Three gyro sensors were placed onboard to read the aircraft angular rates; respectively the roll, pitch, and yaw angular rates. Rotational motion about the sensor's input axis produced a voltage proportional to the rate of rotation. Figure 3.5.11 shows the on-board aircraft gyro box that housed the three gyros and three axis accelerometer package.

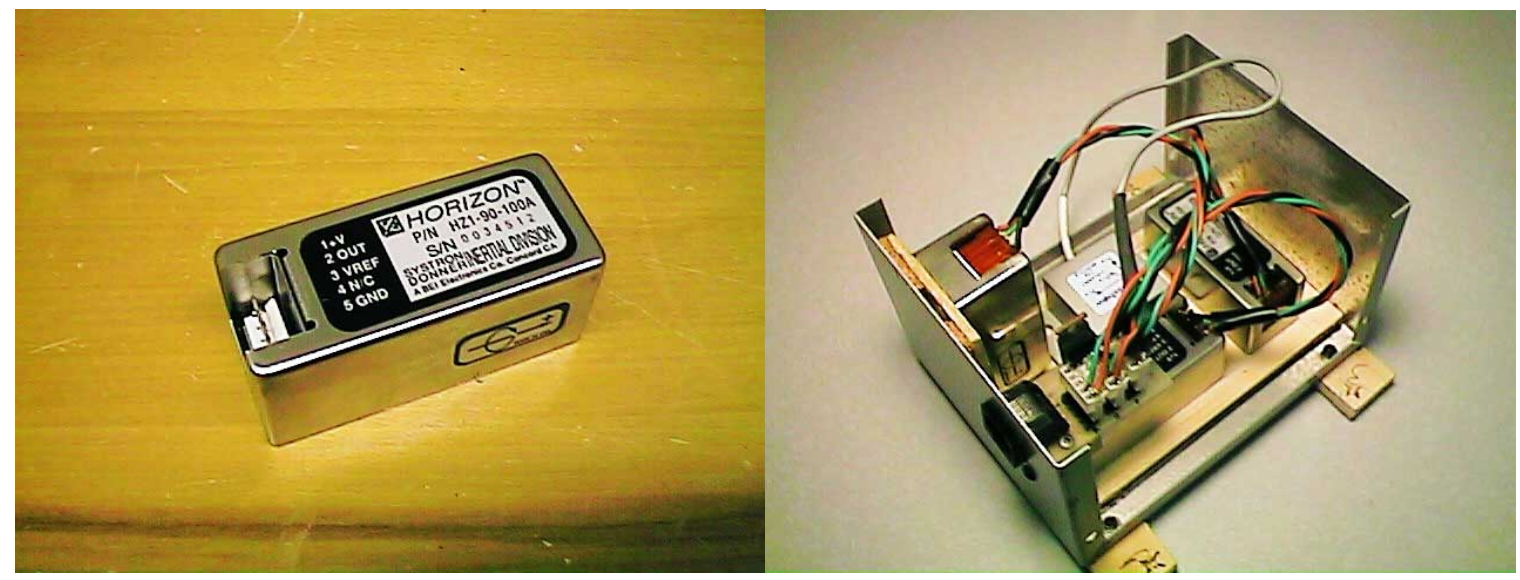

Figure 3.5.10 BEI GyroChip ${ }^{\mathrm{TM}}$ Horizon (angular rate sensor)
Figure 3.5.11 On board gyro box and accelerometer configuration 
Table 3.5.2 provides a few specifications listed from the manufacture's documentation:

Table 3.5.2 - BEI GyroChip ${ }^{\mathrm{TM}}$ Horizon Specifications

\begin{tabular}{|c|c|}
\hline Standard Range & \pm 90 degrees/sec \\
\hline Nominal Output & +0.5 to $+4.5 \mathrm{Vdc}$ \\
\hline Operating Temperature Range & -25 to +70 degree $\mathrm{C}$ \\
\hline Supply Voltage & $+5 \pm 0.25$ volts \\
\hline Shock & $200 \mathrm{~g}$ \\
\hline
\end{tabular}

During flight testing operations one of the gyro's failed and had to be replaced mid-way through the flight testing activities with a BEI GyroChip ${ }^{\text {TM }}$ Model AQRS.

The CXL04M3, M Series three-axis accelerometer (Figure 3.5.12), measures the normal, longitudinal and lateral accelerations of the aircraft. The unit was manufactured and marketed by Crossbow as a general purpose, linear acceleration and/or vibration sensor, which works in a range of $\pm 4 \mathrm{~g}$. This particular accelerometer has a sensing element with a silicon micromachined capacitive beam. The accelerometer offers a wide dynamic range, desirable frequency response and operates on a single $+5 \mathrm{Vdc}$ power supply.

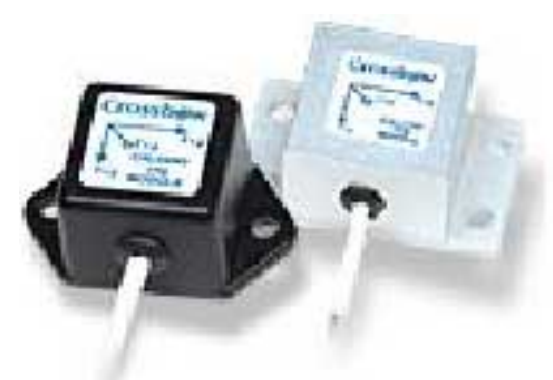

Figure 3.5.12 CXL04M3 accelerometer (aluminum version used, far right) 
Table 3.5.3 provides the specifications and calibration data used for the accelerometer.

Table 3.5.3 - CXL04M3 accelerometer specifications

\begin{tabular}{|c|c|}
\hline Range & $\pm 4 \mathrm{~g}$ \\
\hline Supply Voltage & $+5 \pm 0.25$ volts \\
\hline Temperature Range & -40 to +85 degree C \\
\hline Noise & $5 \mathrm{mGrms}$ \\
\hline Zero g Output (X axis) / Sensitivity & $+2.556 / 0.510$ \\
\hline Zero g Output (Y axis) / Sensitivity & $+2.546 / 0.511$ \\
\hline Zero g Output (Z axis) / Sensitivity & $+2.534 / 0.497$ \\
\hline Options added to model & DC coupled and Aluminum Case \\
\hline
\end{tabular}

From Table 3.5.3, the zero-g voltage was the output voltage of the sensor with zero applied acceleration measured at the factory on the day of calibration.

The aircraft structure vibrates as a result of mainly two factors: the roughly paved runway and the propagation of vibrations from the two aircraft engines. Using isolated mounts, care was taken in mounting the engines to attempt to reduce any induced engine vibration. Other than the vibrations that can be seen in the lower frequency range, it is assumed that there are higher frequency ranges that can not be resolved within the 100 $\mathrm{Hz}$ sampling rate. From flight data, angular rates, with an engine on condition was found to be \pm one degree error, which was considered to be an acceptable range.

\section{Airspeed and Altitude Sensors}

Figure 3.5.13 shows a photo of the Pitot-Static Probe purchased from United Sensor Division. This particular model, PBE-8-H-5-M, had a reinforced tubing extension with an attached mounting chuck, which measures the total and static pressure at the same point within a moving fluid. For the pressure sensors, two Omega ${ }^{\circledR}$ solid-state piezoresistive devices were used, where the sensing diaphragm with implanted resistors is an integral part of the chip. Pressure measurements are recorded, along with the ground temperature for calculating the dynamic pressure, flow velocity and aircraft altitude. The probe was eight inches in length with a 5-inch reinforcement section made of stainless 
steel. Figure 3.5.14 shows a photo of the two pressure sensors that are attached to the probe, which read static and dynamic pressures.

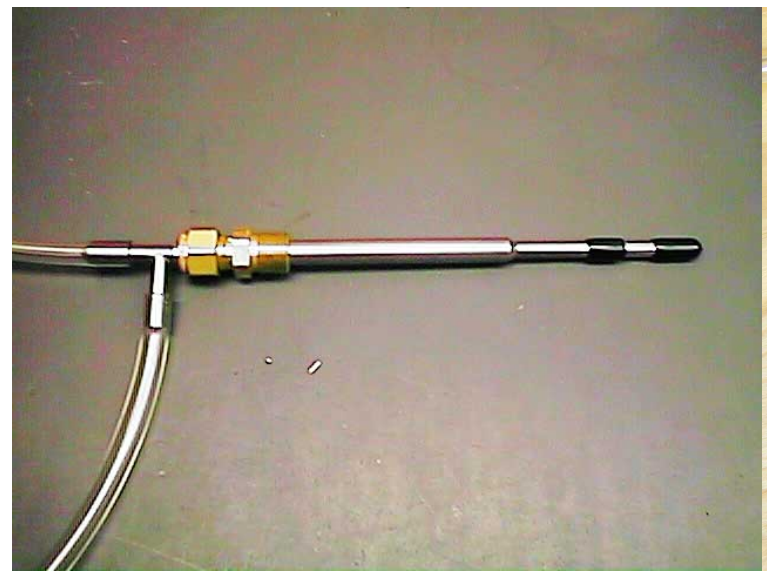

Figure 3.5.13 Pitot-static probe

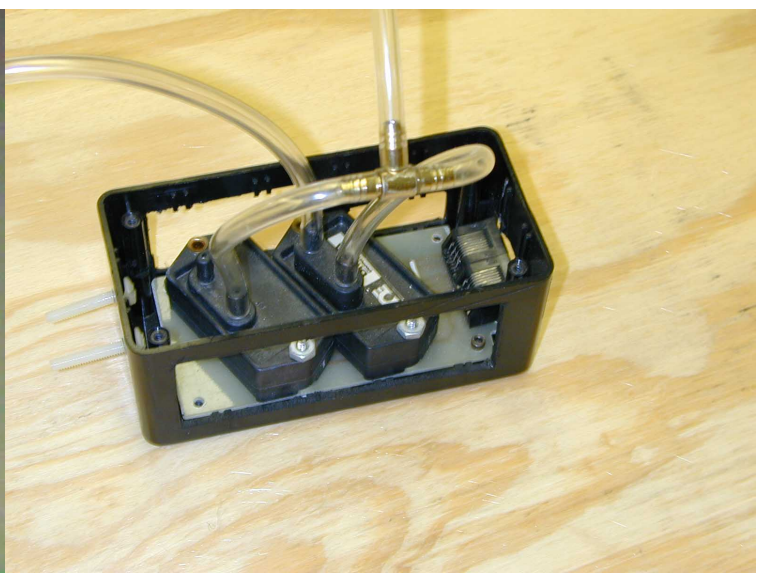

Figure 3.5.14 Pressure sensor box

The pressure transducer that measured the absolute pressure had a range of 0-15 psi, and the second differential pressure sensor had a range of 0-10 inches of $\mathrm{H}_{2} 0$. Typically aircraft velocities during test flight maneuvers fell within a range of 90 to $110 \mathrm{feet} / \mathrm{sec}$. For an average maneuver speed of $100 \mathrm{ft} / \mathrm{sec}$, for a sensor with $\pm 1 \%$ linearity, an accuracy of within 2 feet/sec was possible for the flight test maneuvers. The altitude flight information was only possible to be accurate within \pm 50 feet for the recorded flight data.

\section{$\underline{\text { Flow angles and Control surface deflections }}$}

Alpha and Beta vanes were added to measure the aircraft angle of attack and sideslip flow angles. The vanes act as an aerodynamic boom rotating about the pivot point in such a way that they can perform a one-degree of freedom rotation. They can essentially be divided into two sections, namely the body and tail section, which act like a miniature weather vane. $20 \mathrm{~K}$ ohms single-turn wire wound potentiometer measured the position of each vane relative to the fuselage centerline. The flow angle potentiometers were attached to the shaft of each vane. Deflections were then translated to the potentiometer rotation subsequently producing the flow angle signal. Figures 3.5.15 and 3.5.16 show the alpha and beta vane assemblies along with the single turn potentiometers housed within the nose section. 


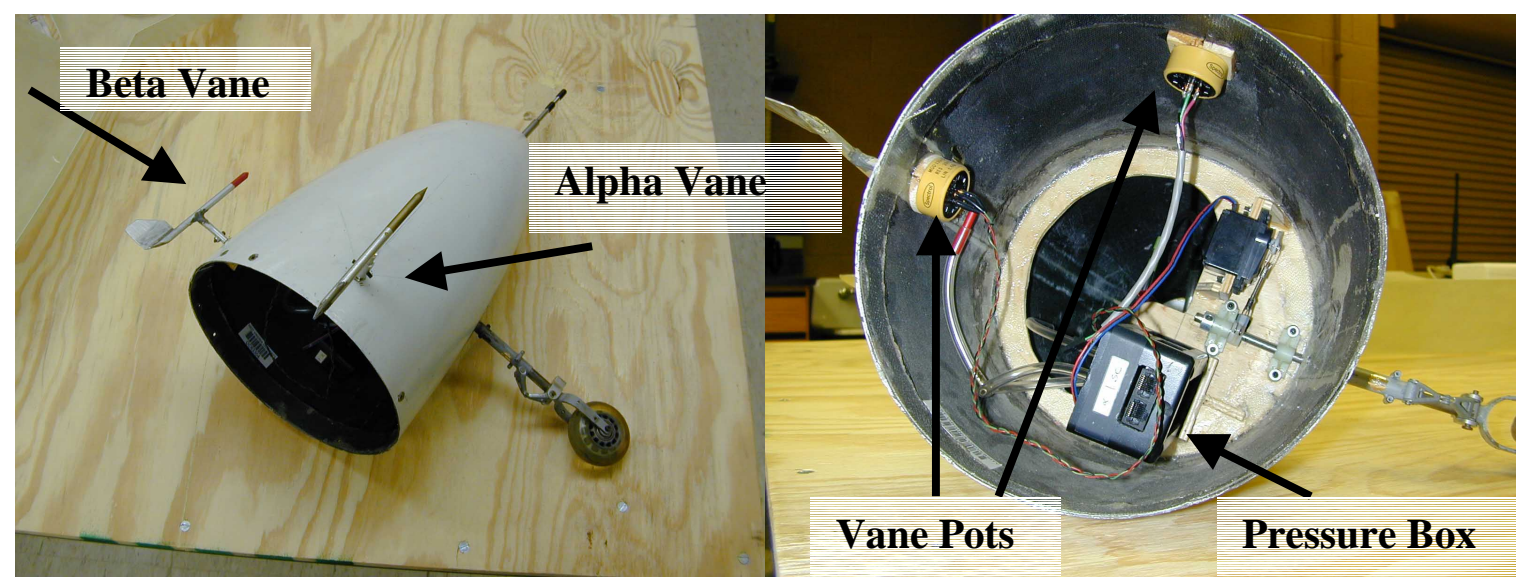

Figure 3.5.15 Alpha and Beta vane

Figure 3.5.16 Angle of Attack and Sideslip vane potentiometers

A smaller version of the single turn potentiometers, example shown in Figure 3.5.17, rated at $10 \mathrm{~K}$ ohms measured the individual surface deflection for each primary control surface. Prior to each test flight, the control surface and flow angle potentiometers were calibrated at the field before launching the vehicle. Figure 3.5.18 provides a view from underneath the tail section of the aircraft, showing the mounting of the rudder, left, and right elevator potentiometers.

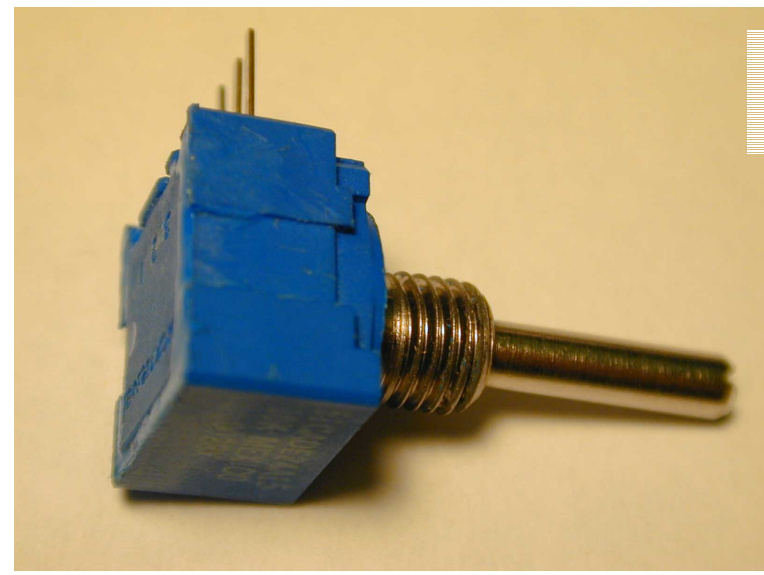

Figure 3.5.17 Control surface potentiometer

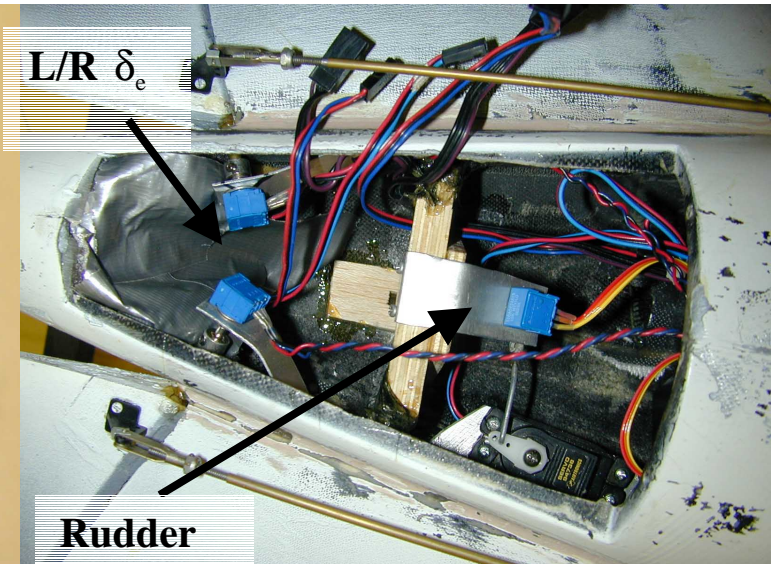

Figure 3.5.18 Elevator and rudder potentiometers

The primary surfaces included measurements for the left and right elevator, left and right ailerons, and rudder control surfaces. Potentiometer measurements from flight test maneuvers did exhibit hysteresis tendencies, in the sense that the elevator did not exactly return to the same exact position. One cause of this would be from the slack or "slop" 
found within the servo linkage connection. A second reason was that the servo itself sometimes does not always return to the exact same starting position.

\subsection{Moments of Inertia}

For PID analysis, a truthful representation of the aircraft inertia data information was required for analysis of the flight test maneuvers. Two methods that are popularly used for this purpose, the first method estimates the values from design data ${ }^{25}$ and the second method uses a pendulum based experimental setup ${ }^{26,27}$. Using the first method, Kirschbaum ${ }^{25}$ described that the measured and calculated moments of inertia could be estimated within 10 percent. For the pendulum-based experimental method Soule ${ }^{27}$ noted that the values could be computed to within $\pm 2.5 \%, \pm 1.3 \%$, and $\pm 0.8 \%$ for the $\mathrm{X}, \mathrm{Y}$, and $\mathrm{Z}$ axes respectively. Overall the pendulum method has been in use for quite some time and was noted to provide accurate results, so a version of this method was used to determine the aircraft values. From a priori values of the dimensions, weight of the pendulum, and the displacement for an undamped pendulum oscillating with small amplitude within a vacuum, Soule and Miller derived the basic equation of motion for the application of the pendulum method as:

$$
\mathrm{I} \frac{\mathrm{d}^{2} \theta_{\mathrm{pe}}}{\mathrm{dt}^{2}}+\mathrm{b}_{\mathrm{pe}} \cdot \theta_{\mathrm{pe}}=0
$$

where $I$ is the moment of inertia about the axis of oscillation, $b_{p}$ is a constant depending on the dimensions and weight of different types of pendulums, and $\theta_{\mathrm{p}}$ is the angular

displacement of the pendulum ${ }^{27}$. From the solution of this general equation, the period of oscillation can be found so that;

$$
\begin{gathered}
T_{p e}=\frac{2 \pi}{\sqrt{b_{p e} / I}} \\
I=\frac{T^{2} b_{p e}}{4 \pi^{2}}
\end{gathered}
$$

To obtain inertia moment values, an experimental setup was built and tested. Figures 3.6.1 and 6.3.2 show laboratory photos of the B777 aircraft installed on the pendulum apparatus. 


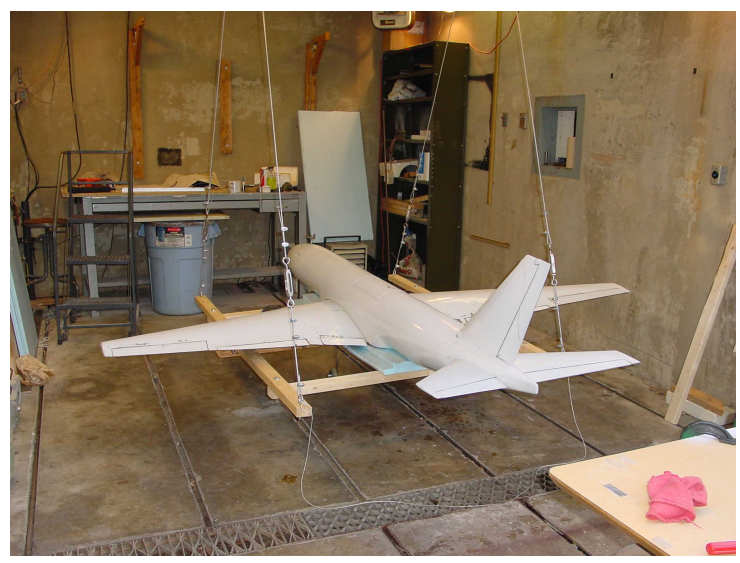

Figure 3.6.1 Pendulum setup (axis-Ix)

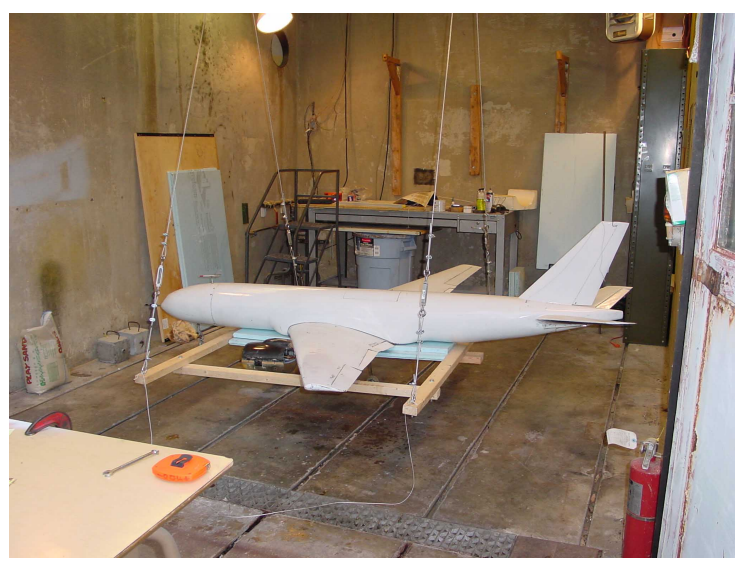

Figure 3.6.2 Pendulum setup (axis-Iy)

For determining the $\mathrm{x}$ and $\mathrm{y}$ axes, a compound pendulum setup was used. Due to size and space constraints, the aircraft was rotated inside of the compound pendulum. For determining the moments of inertia on the z-axis, a bifilar torsion type of pendulum was created. For the z-axis, the axis of oscillation is vertical and for the compound pendulum the axis of oscillation is horizontal and passes through the points of the support. The final apparatus was adjusted from the original technical note with respect to how the aircraft was attached to the pendulum, cabling system, and the overall mechanical connection point. The pendulum itself, on the axis of oscillation was attached with machined disks that fit through a steel bar, which supported the weight of the pendulum and aircraft model. Each disk was lubricated to provide the most reasonable "friction reduced" connection possible. In the original technical note, the overhead connections used universal joints or knife-edge attachments with the aircraft. Since the model was unable to be directly attached by the wings to the pendulum, a wooden platform supported the aircraft from underneath to perform the test experiments. The wire cabling could be adjusted to various lengths to level the platform, which were then attached to the wooden base support frame. Overall the total weight was collected for the pendulum (including the cradle, cabling, etc.) as well as the weight of the overall aircraft. To obtain the $\mathrm{z}$-axis measurements, the platform was then lowered to house the additional drop down connection points. Several tests were performed for each of the axis measurements and the average time was used in the calculations to produce the final moment of inertia values. Using the apparatus geometry and the average time of oscillation a set of final values were calculated for the B777 previously shown in section 3.2, Table 3.2.2. 


\section{Chapter 4}

\section{Aircraft Equations of Motion and Mathematical Modeling}

\subsection{Review of the Equations of Motion}

The aircraft dynamic equations are to be modeled in polar coordinate form using the body axis equations of motion ${ }^{17,28,29,30}$. A non-rotating Earth fixed axis system specific to a point on the ground is chosen as an inertial reference point. There are several major assumptions:

1) The mass of the aircraft remains constant with respect to time.

2) The mass distribution of the aircraft remains constant with time for the duration of the PID maneuver.

3) The aerodynamic and thrust forces are assumed to be the only external forces acting upon the aircraft.

4) The XZ plane as the plane of symmetry for the aircraft.

5) The aircraft is a rigid body.

The aircraft equations of motion are derived from Newton's second law in terms of the conservation of linear and angular momentum. Using the application of the conservation of linear and angular momentum lead to the following equations (4.1.1 - 4.1.6). With respect to the body axis system:

$$
\begin{aligned}
& \mathrm{m}(\dot{\mathrm{U}}-\mathrm{VR}+\mathrm{WQ})=\mathrm{mg}_{\mathrm{X}}+\mathrm{F}_{\mathrm{AX}}+\mathrm{F}_{\mathrm{TX}} \\
& \mathrm{m}(\dot{\mathrm{V}}-\mathrm{UR}+\mathrm{WP})=\mathrm{mg}_{\mathrm{Y}}+\mathrm{F}_{\mathrm{AY}}+\mathrm{F}_{\mathrm{TY}} \\
& \mathrm{m}(\dot{\mathrm{W}}-\mathrm{UQ}+\mathrm{VP})=\mathrm{mg}_{\mathrm{Z}}+\mathrm{F}_{\mathrm{AZ}}+\mathrm{F}_{\mathrm{TZ}}
\end{aligned}
$$

Using the products and moments of inertia:

$$
\begin{aligned}
& \mathrm{I}_{\mathrm{xx}} \dot{\mathrm{P}}-\mathrm{I}_{\mathrm{xz}} \dot{\mathrm{R}}-\mathrm{I}_{\mathrm{xz}} \mathrm{PQ}+\left(\mathrm{I}_{\mathrm{zz}}-\mathrm{I}_{\mathrm{yy}}\right) \mathrm{RQ}=\mathrm{L}_{\mathrm{A}}+\mathrm{L}_{\mathrm{T}} \\
& \mathrm{I}_{\mathrm{yy}} \dot{\mathrm{Q}}+\left(\mathrm{I}_{\mathrm{xx}}-\mathrm{I}_{\mathrm{zz}}\right) \mathrm{PR}+\mathrm{I}_{\mathrm{xz}}\left(\mathrm{P}^{2}-\mathrm{R}^{2}\right)=\mathrm{M}_{\mathrm{A}}+\mathrm{M}_{\mathrm{T}} \\
& \mathrm{I}_{\mathrm{zz}} \dot{\mathrm{R}}-\mathrm{I}_{\mathrm{xz}} \dot{\mathrm{P}}+\left(\mathrm{I}_{\mathrm{yy}}-\mathrm{I}_{\mathrm{xx}}\right) \mathrm{PQ}+\mathrm{I}_{\mathrm{xz}} \mathrm{QR}=\mathrm{N}_{\mathrm{A}}+\mathrm{N}_{\mathrm{T}}
\end{aligned}
$$

Figure 4.1.1 shows a general graphical representation of the body axis system with forces and moments acting on the aircraft. 


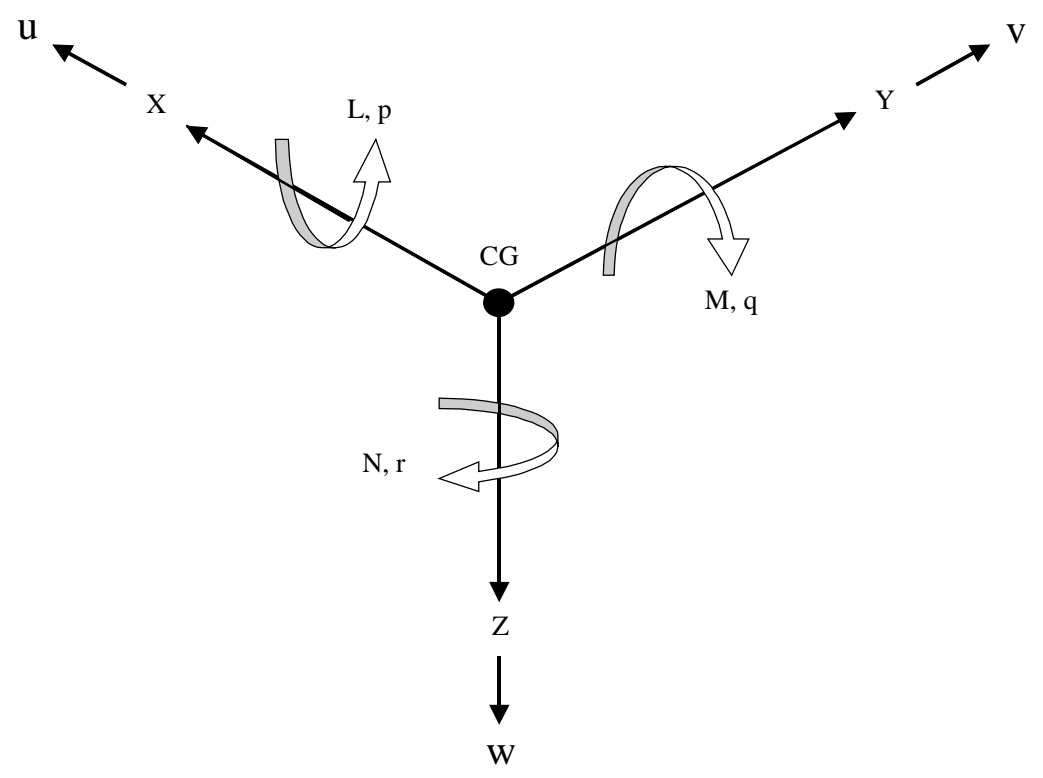

\section{Figure 4.1.1 Body axis system with forces and moments acting on the aircraft}

These equations describe the aircraft motion with respect to the rotational body axis (XYZ). The result is a set of non-linear system of equations to be solved in terms of velocity components and angular rates. To solve for these components it is necessary to describe the relative orientation of the XYZ body axis to the inertial Earth fixed $\mathrm{X}^{\prime} \mathrm{Y}^{\prime} \mathrm{Z}^{\prime}$ using the Euler angles ${ }^{28}$. Since $X_{1} Y_{1} Z_{1}$ and $X^{\prime} Y^{\prime} Z^{\prime}$ are parallel to each other it follows that:

$$
\mathrm{U}_{1}=\dot{\mathrm{x}} \quad \mathrm{V}_{1}=\dot{\mathrm{y}} \quad \mathrm{W}_{1}=\dot{\mathrm{z}}
$$

1) Consider $X^{\prime} Y^{\prime} Z^{\prime}$ translated parallel to itself so that the origin will coincide with the aircraft center of gravity and rename the axis $\mathrm{X}_{1} \mathrm{Y}_{1} \mathrm{Z}_{1}$.

2) System $X_{1} Y_{1} Z_{1}$ is then rotated about $Z_{1}$ by an angle $\psi$ to generate $X_{2} Y_{2} Z_{2}$.

3) System $X_{2} Y_{2} Z_{2}$ is then rotated about $Y_{2}$ by an angle $\theta$ to generate $X_{3} Y_{3} Z_{3}$.

4) System $X_{3} Y_{3} Z_{3}$ is then rotated about $X_{3}$ by an angle $\phi$ to generate the initial body axis XYZ. 
The angles $\varphi, \theta$ and $\phi$ are referred to as the Euler angles. Figure 4.1.2 shows a graphical representation of this transformation process.

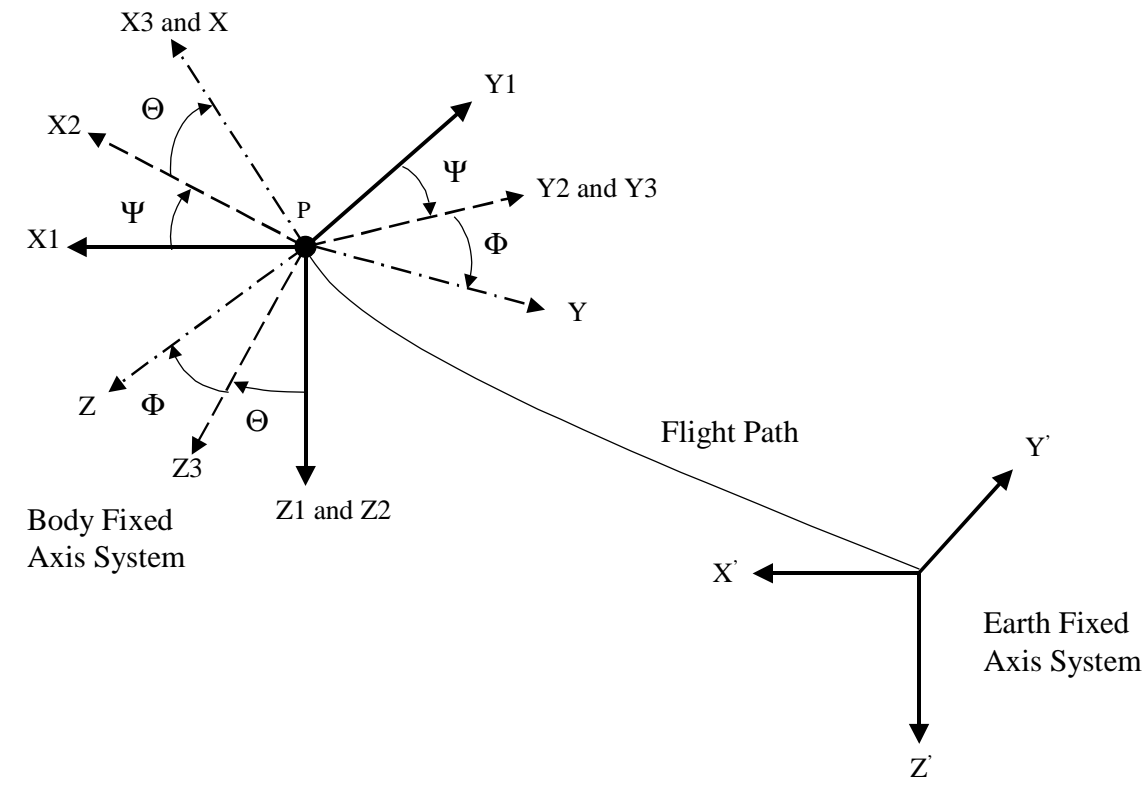

\section{Figure 4.1.2 Geometric description of aircraft orientation with respect to an Earth fixed inertial axis coordinate system}

Using the Euler angles it is then possible to describe the flight path of the aircraft using the velocity components $\mathrm{U}, \mathrm{V}$ and $\mathrm{W}$. Completing the graphical description above, we obtain a matrix buildup relating the Euler angles and velocity components.

$$
\left[\begin{array}{c}
\mathrm{U}_{1} \\
\mathrm{~V}_{1} \\
\mathrm{~W}_{1}
\end{array}\right]=\left[\begin{array}{ccc}
\cos \psi & -\sin \psi & 0 \\
\sin \psi & \cos \psi & 0 \\
0 & 0 & 1
\end{array}\right]\left[\begin{array}{ccc}
\cos \theta & 0 & \sin \theta \\
0 & 1 & 0 \\
-\sin \theta & 0 & \cos \theta
\end{array}\right]\left[\begin{array}{ccc}
1 & 0 & 0 \\
0 & \cos \phi & -\sin \phi \\
0 & \sin \phi & \cos \phi
\end{array}\right]\left[\begin{array}{c}
\mathrm{U} \\
\mathrm{V} \\
\mathrm{W}
\end{array}\right]
$$

The next step is describing the angular rates as a function of the Euler angles.

$$
\begin{gathered}
{\left[\begin{array}{c}
\mathrm{p} \\
\mathrm{q} \\
\mathrm{r}
\end{array}\right]=\left[\begin{array}{c}
\dot{\phi} \\
0 \\
0
\end{array}\right]+\left[\begin{array}{ccc}
1 & 0 & 0 \\
0 & \cos \phi & \sin \phi \\
0 & -\sin \phi & \cos \phi
\end{array}\right]\left[\begin{array}{l}
0 \\
\dot{\theta} \\
0
\end{array}\right]+\left[\begin{array}{ccc}
1 & 0 & 0 \\
0 & \cos \phi & \sin \phi \\
0 & -\sin \phi & \cos \phi
\end{array}\right]\left[\begin{array}{ccc}
\cos \theta & 0 & -\sin \theta \\
0 & 1 & 0 \\
\sin \theta & 0 & \cos \theta
\end{array}\right]\left[\begin{array}{c}
0 \\
0 \\
\dot{\varphi}
\end{array}\right]} \\
{\left[\begin{array}{l}
\mathrm{p} \\
\mathrm{q} \\
\mathrm{r}
\end{array}\right]=\left[\begin{array}{ccc}
1 & 0 & -\sin \theta \\
0 & \cos \phi & \sin \phi \cos \theta \\
0 & -\sin \phi & \cos \phi \cos \theta
\end{array}\right]\left[\begin{array}{c}
\dot{\phi} \\
\dot{\theta} \\
\dot{\psi}
\end{array}\right]}
\end{gathered}
$$


This matrix expression can then be inverted to solve for the angular velocities.

$$
\left[\begin{array}{c}
\dot{\phi} \\
\dot{\theta} \\
\dot{\psi}
\end{array}\right]=\left[\begin{array}{ccc}
1 & 0 & -\sin \theta \\
0 & \cos \phi & \sin \phi \cos \theta \\
0 & -\sin \phi & \cos \phi \cos \theta
\end{array}\right]^{-1}\left[\begin{array}{l}
\mathrm{p} \\
\mathrm{q} \\
\mathrm{r}
\end{array}\right]
$$

The inversion of the matrix provides the following relationships:

$$
\begin{gathered}
\dot{\phi}=\mathrm{p}+\mathrm{q} \sin \phi \tan \theta+\mathrm{r} \cos \phi \tan \theta \\
\dot{\theta}=\mathrm{q} \cos \theta-\mathrm{r} \sin \phi \\
\dot{\psi}=(\mathrm{q} \sin \phi+\mathrm{r} \cos \phi) \sec \theta
\end{gathered}
$$

The above relationships are known as the aircraft kinematic equations. Figure 4.1.3 below shows a graphical basis for expressing the equations of motion in a polar coordinate form.

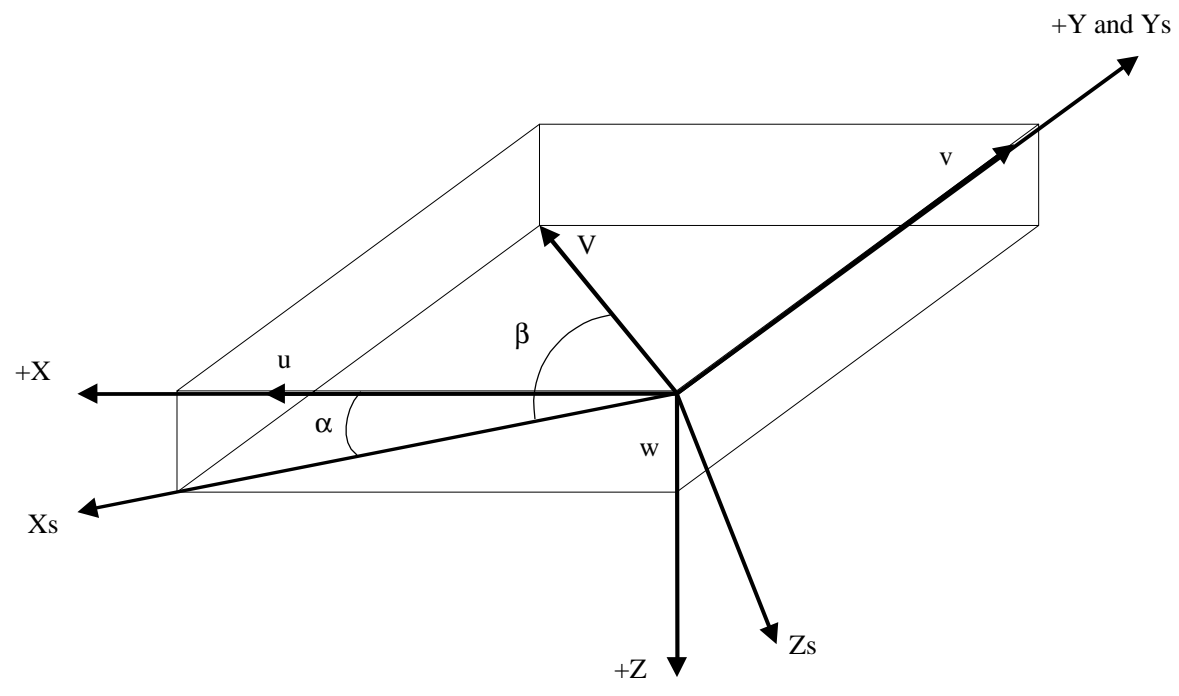

\section{Figure 4.1.3 Graphical basis for expressing the equations of motion in a polar coordinate form}

For the polar coordinate system, it necessary to express the terms of angle of attack, sideslip angle and aircraft velocity. Each of these values can be directly measured from the aircraft. A primary disadvantage of the $(\alpha, \beta, \mathrm{V})$ system is that it is singular at zero velocity, where alpha and beta are not defined ${ }^{10}$. However, this is only a concern when 
the vehicle is hovering, which is not the case for this aircraft. The final expressions for the equations of motion are provided below ${ }^{7,10,28}$.

$$
\begin{gathered}
\dot{\mathrm{V}}=\frac{\overline{\mathrm{q}} \mathrm{m}}{\mathrm{m}} \mathrm{C}_{\mathrm{D}_{\mathrm{w}}}+\mathrm{g}(\cos \phi \cos \theta \sin \alpha \cos \beta+\sin \phi \cos \theta \sin \beta- \\
\sin \theta \cos \alpha \cos \beta) \\
\dot{\alpha}=\mathrm{q}-\tan \beta(\mathrm{p} \cos \alpha+\mathrm{r} \sin \alpha)-\frac{\overline{\mathrm{q} S}}{\mathrm{mV} \cos \beta} \mathrm{C}_{\mathrm{L}}+ \\
\frac{\mathrm{g}}{\mathrm{V} \cos \beta}(\cos \theta \cos \phi \cos \alpha+\sin \theta \sin \alpha) \\
\dot{\mathrm{p}}=\mathrm{p} \sin \alpha-\mathrm{r} \cos \alpha+\frac{\overline{\mathrm{q}} \mathrm{m}}{\mathrm{mV}} \mathrm{C}_{\mathrm{Yw}}+\frac{\mathrm{g}}{\mathrm{V}} \cos \beta \cos \theta \sin \phi- \\
\frac{\mathrm{g}}{\mathrm{V}} \sin \beta(\cos \theta \cos \phi \sin \alpha-\sin \theta \cos \alpha) \\
\dot{\mathrm{q}}=\frac{1}{\mathrm{I}_{\mathrm{xy}}}\left[\mathrm{I}_{\mathrm{xy}}(\dot{\mathrm{q}}-\mathrm{pr})+\mathrm{I}_{\mathrm{xz}}(\dot{\mathrm{r}}+\mathrm{pq})+\mathrm{qr}\left(\mathrm{I}_{\mathrm{yy}}-\mathrm{I}_{\mathrm{zz}}\right)+\mathrm{I}_{\mathrm{yz}}\left(\mathrm{q}^{2}-\mathrm{r}^{2}\right)+\overline{\mathrm{q}} \mathrm{SbC}_{1}\right] \\
\dot{\mathrm{r}}=\frac{1}{\mathrm{I}_{\mathrm{zz}}}\left[\mathrm{I}_{\mathrm{xz}}(\dot{\mathrm{p}}-\mathrm{qr})+\mathrm{I}_{\mathrm{yz}}(\dot{\mathrm{r}}-\mathrm{pq})+\mathrm{rp}\left(\mathrm{I}_{\mathrm{zz}}-\mathrm{I}_{\mathrm{xx}}\right)+\mathrm{I}_{\mathrm{xz}}\left(\mathrm{r}^{2}-\mathrm{p}^{2}\right)+\overline{\mathrm{q}}+\mathrm{Sr} \overline{\mathrm{c}} \mathrm{C}_{\mathrm{m}}\right]
\end{gathered}
$$

Modeling from this set of classical non-linear longitudinal and lateral-directional equations of motion defines the stability axis force coefficients as

$$
\begin{aligned}
& C_{L}=C_{N} \cos \alpha-C_{A} \sin \alpha \\
& C_{D}=C_{A} \cos \alpha+C_{N} \sin \alpha
\end{aligned}
$$

and the wind axis coefficients as:

$$
\begin{aligned}
& C_{D_{\mathrm{w}}}=C_{D} \cos \beta-C_{Y} \sin \beta \approx C_{D} \\
& C_{Y_{\mathrm{W}}}=C_{Y} \cos \beta+C_{D} \sin \beta \approx C_{Y}
\end{aligned}
$$




\subsection{Longitudinal and Lateral-Directional Mathematical Modeling}

The linear build-up used for modeling the total, longitudinal non-dimensional aerodynamic force and moment coefficients is shown in the following equations:

$$
\begin{aligned}
& \mathrm{C}_{\mathrm{N}}=\mathrm{C}_{\mathrm{N}_{0}}+\mathrm{C}_{\mathrm{N}_{\alpha}} \alpha+\mathrm{C}_{\mathrm{N}_{\mathrm{q}}} \frac{\mathrm{q} \overline{\mathrm{c}}}{2 \mathrm{VR}}+\mathrm{C}_{\mathrm{N}_{\delta_{\mathrm{e}}}} \delta_{\mathrm{e}} \\
& \mathrm{C}_{\mathrm{A}}=\mathrm{C}_{\mathrm{A}_{0}}+\mathrm{C}_{\mathrm{A}_{\alpha}} \alpha+\mathrm{C}_{\mathrm{A}_{\mathrm{q}}} \frac{\mathrm{q} \overline{\mathrm{c}}}{2 \mathrm{VR}}+\mathrm{C}_{\mathrm{A}_{\delta_{\mathrm{e}}}} \delta_{\mathrm{e}} \\
& \mathrm{C}_{\mathrm{m}}=\mathrm{C}_{\mathrm{m} 0}+\mathrm{C}_{\mathrm{m} \alpha} \alpha+\mathrm{C}_{\mathrm{m}_{\mathrm{q}}} \frac{\mathrm{q} \overline{\mathrm{c}}}{2 \mathrm{VR}}+\mathrm{C}_{\mathrm{m}_{\mathrm{e}_{\mathrm{e}}}} \delta_{\mathrm{e}}
\end{aligned}
$$

It is assumed that the longitudinal aerodynamic coefficients are functions of the aerodynamic bias (where $\mathrm{C}_{\mathrm{N}_{\mathrm{o}}}=\left.\mathrm{C}_{\mathrm{N}}\right|_{\alpha=\mathrm{q}=\delta_{\mathrm{e}}=0}$ ), angle of attack, pitch rate, and the longitudinal elevator control surfaces. The lateral-directional aerodynamic coefficients are functions of the aerodynamic bias, sideslip angle, roll rate, yaw rate, as well as lateral-directional aileron and rudder control surfaces;

$$
\begin{aligned}
& \mathrm{C}_{\mathrm{Y}}=\mathrm{C}_{\mathrm{Y}_{0}}+\mathrm{C}_{\mathrm{Y} \beta} \beta+\mathrm{C}_{\mathrm{Yp}} \frac{\mathrm{pb}}{2 \mathrm{VR}}+\mathrm{C}_{\mathrm{Y}_{\mathrm{r}}} \frac{\mathrm{rb}}{2 \mathrm{VR}}+\mathrm{C}_{\mathrm{Y}_{\delta_{\mathrm{a}}}} \delta_{\mathrm{a}}+\mathrm{C}_{\mathrm{Y}_{\delta_{\mathrm{r}}}} \delta_{\mathrm{r}} \\
& \mathrm{C}_{1}=\mathrm{C}_{1_{0}}+\mathrm{C}_{1 \beta} \beta+\mathrm{C}_{\mathrm{l}_{\mathrm{p}}} \frac{\mathrm{pb}}{2 \mathrm{VR}}+\mathrm{C}_{\mathrm{l}_{\mathrm{r}}} \frac{\mathrm{rb}}{2 \mathrm{VR}}+\mathrm{C}_{\mathrm{l}_{\mathrm{a}}} \delta_{\mathrm{a}}+\mathrm{C}_{1_{\delta_{\mathrm{r}}}} \delta_{\mathrm{r}} \\
& \mathrm{C}_{\mathrm{n}}=\mathrm{C}_{\mathrm{n}_{0}}+\mathrm{C}_{\mathrm{n}_{\beta} \beta} \beta+\mathrm{C}_{\mathrm{n}_{\mathrm{p}}} \frac{\mathrm{pb}}{2 \mathrm{VR}}+\mathrm{C}_{\mathrm{n}_{\mathrm{r}}} \frac{\mathrm{rb}}{2 \mathrm{VR}}+\mathrm{C}_{\mathrm{n}_{\mathrm{a}_{\mathrm{a}}}} \delta_{\mathrm{a}}+\mathrm{C}_{\mathrm{n}_{\delta_{\mathrm{r}}}} \delta_{\mathrm{r}}
\end{aligned}
$$

where the subscripts indicate derivatives with respect to the subscript quantity ${ }^{17}$. The research objective was to provide estimates for the right-hand-side (RHS) coefficients in equations (4.2.1-4.2.6). 


\section{Chapter 5}

\section{Parameter Estimation Method and pEst Program Operation}

\subsection{Maximum Likelihood Method and Newton-Raphson Minimization}

The Maximum Likelihood method coupled with a Newton-Raphson minimization technique has been one of the most successful Parameter Identification (PID) methods. This approach, introduced at NASA Dryden in the late 1960's, is well documented and has yielded excellent results for a large variety of research aircraft ${ }^{1,3,7,10,17,29,30}$. The ML estimates obtained from this method have three asymptotic properties: they are unbiased, follow a Gaussian distribution, and feature the lowest possible variance ${ }^{31}$. By asymptotic, it implies that the aforementioned estimate properties are true if infinite data time is available. However, these estimate properties are best approximated if the data time is long enough (ie. a couple of periods of the lowest system natural frequency) ${ }^{31}$.

The ML method allows for minimizing a quadratic cost function containing differences between the aircraft measured and computed responses. In general, the goal is to maximize the probability that the computed system responses, based on a set of estimated stability derivatives, are representative of the true system dynamics. Thus, the objective was to maximize the probability that the estimated stability derivatives are representative of the true aircraft dynamics. The conditional probability, which is denoted as $\mathrm{P}(\mathrm{z} / \xi)$, known as the likelihood function is defined as:

$$
\mathrm{P}(\mathrm{z} / \xi)=\frac{1}{2 \pi^{\left(\frac{\mathrm{n}_{\mathrm{t}} \mathrm{n}_{\mathrm{Z}}}{2}\right)}\left|\mathrm{W}_{1}^{-1}\right|^{-\frac{\mathrm{n}_{\mathrm{t}}}{2}}} \mathrm{e}^{-\mathrm{J}(\xi)}
$$

and $\mathrm{P}(\xi)$ is defined as:

$$
\mathrm{P}(\xi)=\frac{1}{2 \pi^{\left(\frac{\mathrm{n} \xi}{2}\right)}\left|\mathrm{W}_{2}-1\right| \frac{1}{2}} \mathrm{e}^{-\frac{1}{2}\left[\left(\xi-\hat{\xi}_{0}\right)^{\mathrm{T}} \mathrm{W}_{2}\left(\xi-\hat{\xi}_{0}\right)\right]}
$$

where

- $\mathrm{n}_{\mathrm{z}}$ is the number of actual and computed responses;

- $\quad n_{\xi}$ is the number of coefficients to be estimated in $\xi$; 
- $\mathrm{n}_{\mathrm{t}}$ is the number of sample times in the time history;

- $\quad \mathrm{W}_{1}$ represents the response error-weighting matrix containing the measurement noise covariance matrix;

- $\quad \mathrm{W}_{2}$ is an "a priori" value weighting matrix;

- $\xi$ represents a vector containing the aerodynamic stability and control derivatives to be estimated;

- $\hat{\xi}_{0}$ represents a vector containing the estimated values for the derivatives, initially set to zero or some random initial value.

This likelihood function is the conditional probability that a response "z" occurs for an actual system for a given value of the unknown parameters contained in the parameter vector to be estimated. All state and measurement noise can be described as a Gaussian white sequence. To simplify the definition above, the $\mathrm{W}_{1}$ matrix allows for the assignment of "relative importance" of a particular response in a particular time history.

$$
\mathrm{P}(\mathrm{z})=\frac{1}{2 \pi^{\left(\frac{\mathrm{n}_{\mathrm{t}} \mathrm{n}_{\mathrm{z}}}{2}\right)}\left|\mathrm{W}_{1}^{-1}\right|^{\frac{\mathrm{n}_{\mathrm{t}}}{2}}} \mathrm{e}^{-\mathrm{J}(\xi)} \frac{1}{2 \pi^{\left(\frac{\mathrm{n}_{\xi}}{2}\right)}\left|\mathrm{W}_{2}^{-1}\right|^{\frac{1}{2}}} \mathrm{e}^{-\frac{1}{2}\left[\left(\xi-\xi_{0}\right)^{\mathrm{T}} \mathrm{W}_{2}\left(\xi-\xi_{0}\right)\right]}
$$

The overall goal was to minimize the cost function $J(\xi)$ in order to maximize the likelihood function. The cost function ${ }^{32}$ is defined as:

$$
\mathrm{J}(\xi)=\frac{1}{2 \mathrm{n}_{\mathrm{t}} \mathrm{n}_{\mathrm{z}}} \sum_{\mathrm{k}=1}^{\mathrm{n}_{\mathrm{t}}}\left[\mathrm{z}\left(\mathrm{t}_{\mathrm{k}}\right)-\mathrm{y}\left(\mathrm{t}_{\mathrm{k}}\right)\right] \mathrm{W}_{1}\left[\mathrm{z}\left(\mathrm{t}_{\mathrm{k}}\right)-\mathrm{y}\left(\mathrm{t}_{\mathrm{k}}\right)\right]
$$

This cost function is then minimized through the application of the NR method ${ }^{1,32,33}$. This method provides a new estimate of the unknown coefficients based on the difference between the actual and computed responses obtained. The maximization of this probability is that the computed responses are representative of the true aircraft dynamics ${ }^{10}$. This NR algorithm solves the associated system of equations using the gradient and the Hessian of the cost function with respect to the vector containing the aerodynamic parameters to be estimated. Setting the gradient with respect to $\xi$, equal to zero the cost function is minimized using: 


$$
\begin{gathered}
\nabla_{\xi} \mathrm{J}\left(\xi_{\mathrm{i}}\right)=\frac{1}{\mathrm{n}_{\mathrm{t}} \mathrm{n}_{\mathrm{z}}} \sum_{\mathrm{k}=1}^{\mathrm{n}_{\mathrm{t}}}\left[\nabla_{\xi \mathrm{y}}\left(\mathrm{t}_{\mathrm{k}}\right)\right]^{\mathrm{T}} \mathrm{W}_{1}\left(\mathrm{z}\left(\mathrm{t}_{\mathrm{k}}\right)-\mathrm{y}\left(\mathrm{t}_{\mathrm{k}}\right)\right) \\
\nabla^{2} \xi \mathrm{J}\left(\xi_{\mathrm{i}}\right)=\frac{1}{\mathrm{n}_{\mathrm{t}} \mathrm{n}_{\mathrm{z}}} \sum_{\mathrm{k}=1}^{\mathrm{n}_{\mathrm{t}}}\left[\nabla_{\xi} \mathrm{y}\left(\mathrm{t}_{\mathrm{k}}\right)\right]^{\mathrm{T}} \mathrm{W}_{1} \nabla_{\xi} \mathrm{y}\left(\mathrm{t}_{\mathrm{k}}\right)
\end{gathered}
$$

leading to:

$$
\xi_{\mathrm{i}+1}=\xi_{1}-\left[\left(\nabla^{2} \xi \mathrm{J}\left(\xi_{\mathrm{i}}\right)\right)\right]^{-1} \nabla_{\xi} \mathrm{J}\left(\xi_{\mathrm{i}}\right)
$$

The process is iterative with the updating of the parameter vector $\xi$ until the convergence criteria is met resulting in the final ML estimates of the aircraft model parameters. Figure 5.1.1 shows a graphical representation of the modified NR minimization technique.

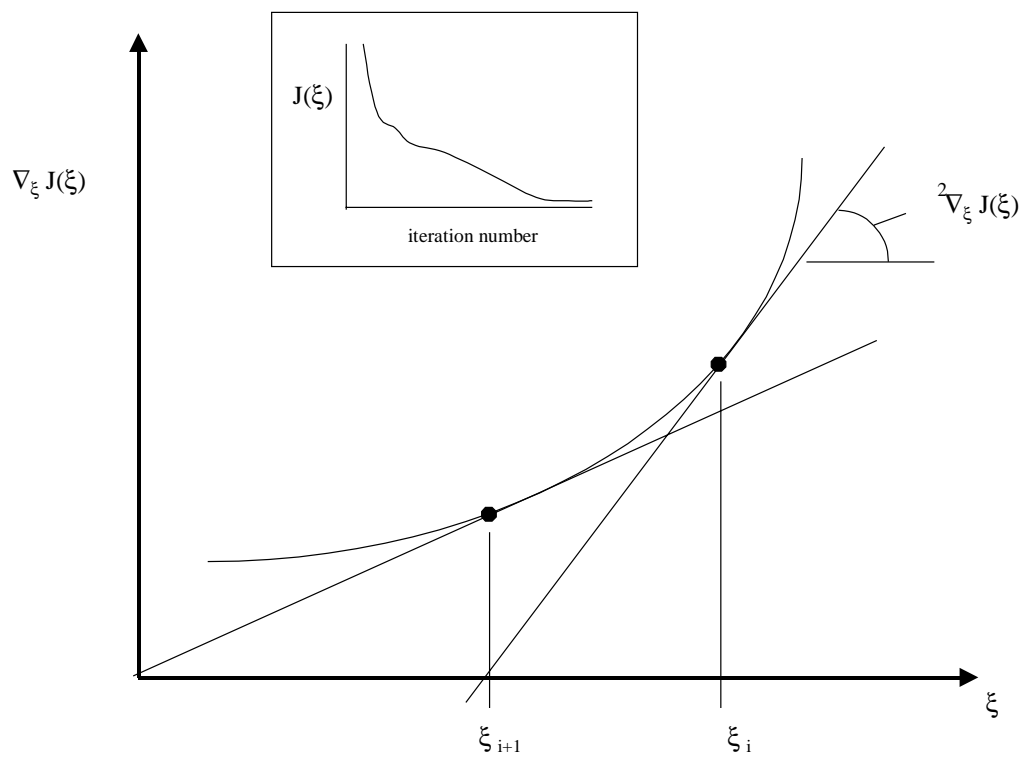

\section{Figure 5.1.1 Graphical representation of the Newton-Raphson minimization technique}

This technique provides a new estimate of the unknown coefficient on the basis of a response error. This response error is the difference between the actual and computed responses. Figure 5.1.2 shows the block diagram of the overall estimation scheme. 


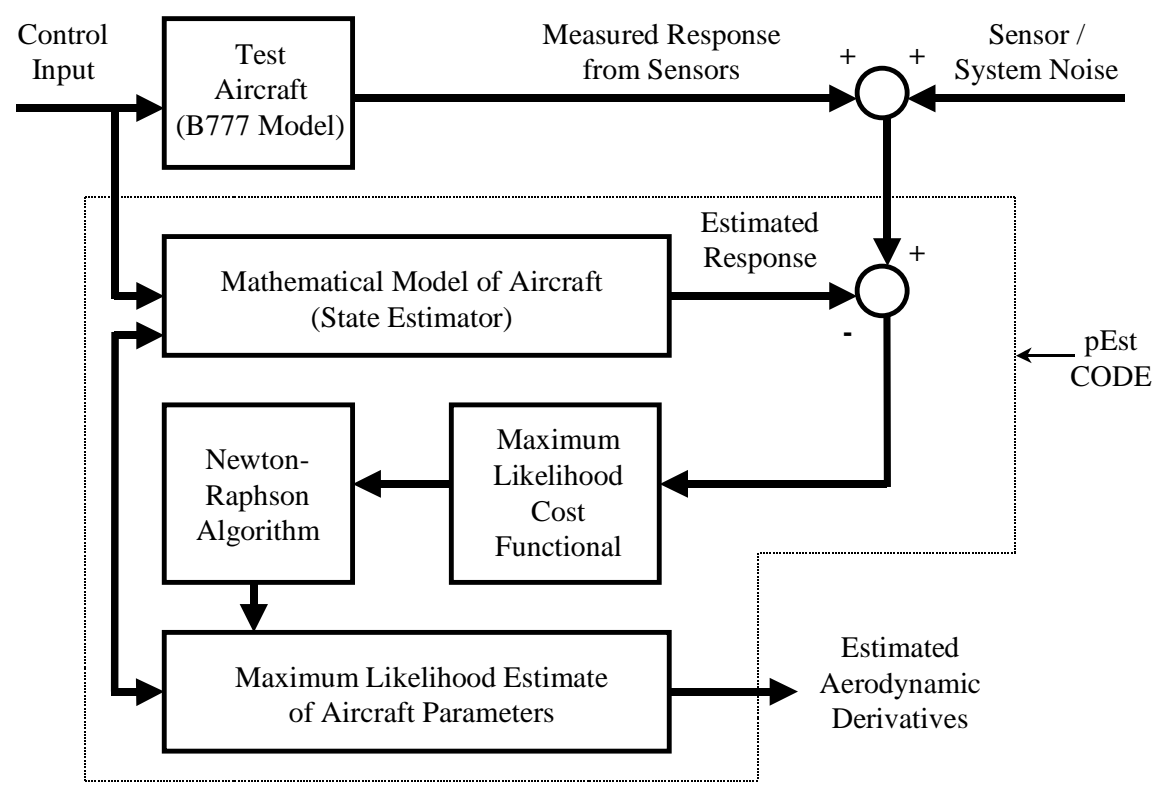

\section{Figure 5.1.2 Block diagram of the parameter identification process}

\section{2 pEst Program Operation}

The pEst program, developed at NASA Dryden Flight Research Center, is a userfriendly interface for determining the stability and control derivatives. The original $\mathrm{pEst}$ package uses a set of ordinary differential equations of motion separated into continuoustime state equations and discrete-time response equations. A pre-determined set of control surface inputs are applied to the true aircraft system and the associated responses are then provided as input to the pEst code. The aircraft dynamics modeled within $\mathrm{pEst}$ are based on the six Degrees Of Freedom (DOF) equations of motion in the polar coordinate form with the state equations being integrated using a fourth order RungeKutta numerical method ${ }^{33}$. The integration method may be specified as either Euler or Runge-Kutta methods. A fourth order Runge-Kutta method was chosen since it is known to be a more accurate even though it can be more computationally more intensive then the euler approach.

The program requires aircraft inputs supplied from a sample flight time history maneuver, start-up information containing initial guesses for estimates, aircraft geometry, and instrumentation sensor locations. These sensor positions correct computed responses 
within the program to include flow-vanes and accelerometers located away from the aircraft's center of gravity (CG). The software supports user interaction, allowing for customization of individual maneuver runs. All time history flight data obtained was separated into individual flight maneuvers for analysis. The user has the capability to choose the stability derivatives to be estimated and also allows for estimates to be held constant during the iterative process. In addition, individual aircraft computed states and responses could be activated or deactivated as needed.

A notable feature of pEst is the capability of evaluating a corresponding CramerRao Bound (CRB) for each derivative. This bound is also known as the standard deviation and the uncertainty level, as a measure of the goodness of the estimates. The $\mathrm{CRB}$ of an estimated scalar parameter is the standard deviation of the error in that parameter $^{32}$. The determination of the Cramer-Rao bounds assumes unbiased estimates with system and measurement noise being modeled as gaussian, independent, white random variables. Considering the case where these assumptions are valid the time history residuals will also be white, random sequences, resulting in a set of Cramer-Rao bounds accurately modeling the scatter of the estimates. In general, the quality of the resulting estimates obtained from the PID process can be evaluated through the inspection of the pEst generated Cramer-Rao bounds and corresponding responses associated with those particular maneuvers.

To account for modeling discrepancies the CRB's are multiplied by a factor of 10 . The practical use for this is to measure the scatter and provide an indication of the estimate accuracy. The actual value may vary, but must remain constant when using several sets of maneuvers. However, since flight data is acquired from a series of maneuvers with changing flight conditions, the estimates can be evaluated for scatter if the derivatives are assumed to change smoothly with the flight conditions ${ }^{31}$. During the evaluation of each data set, the program operates upon a convergence bound criterion for each iterative step:

$$
\text { CONVERGENCE }=\left|\frac{\text { OLD } \cos t-\cos t}{\cos t}\right|<\text { bound }
$$

This bound is the change in the cost between two iterative steps when the default convergence is set to 0.0001 . 


\section{Chapter 6}

\section{Parameter Estimation Results from Collected Flight Data}

\subsection{Flight Testing Activities}

The flight testing activities were carried out at the Louis Bennett Field, located at the WVU Jackson's Mill Conference Center, Jane Lew, WV. This facility was secluded from commercial and general aviation air traffic activity; thus, perfectly suited for research flight testing activities. This facility features a 3,200 feet long, 50 feet wide semi paved runway. For transportation between WVU and the airfield, a customized cradle system (shown in Figure 6.1.1) was designed and built to allow a safe transportation of the model to the flight testing facility.

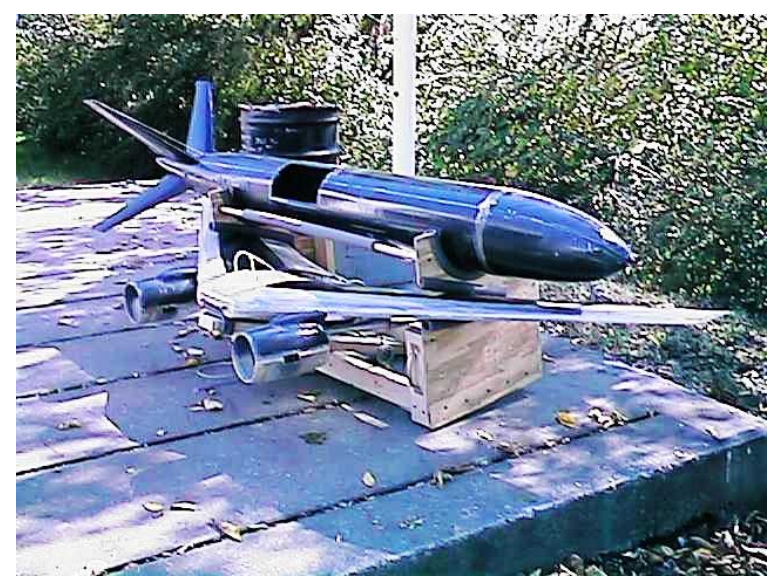

\section{Figure 6.1.1 Cradle transportation unit}

\section{Start of Phase \#1, objective (1) testing}

Testing was completed in multiple stages to minimize the risk to the aircraft. Phase \#1, objective (1) involved the initial ground and taxi tests and to evaluate ground handling qualities and radio system. This stage was also used to test the airframe strength and durability on the rough semi-paved runway, with special attention to the landing gear setup. The landing gear was a conventional three-gear configuration that includes one nose wheel and two mains, shown in Figure 6.1.2. 


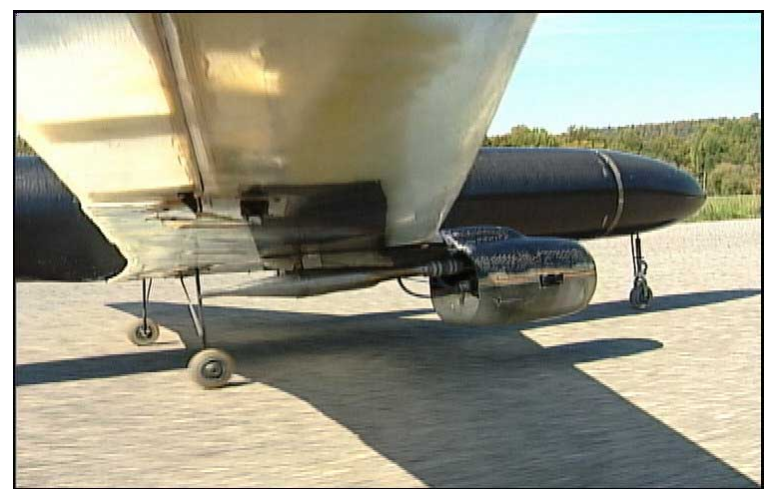

Figure 6.1.2 Landing gear configuration

For testing of the landing gear, roller-blade wheels were able to provide a smooth motion and handle the rough surface. The only minor adjustments were necessary with the wing attachment points of the gear legs. The main gear attachment used both fiberglass and aluminum support straps to connect directly to wing section shown in Figure 6.1.3.

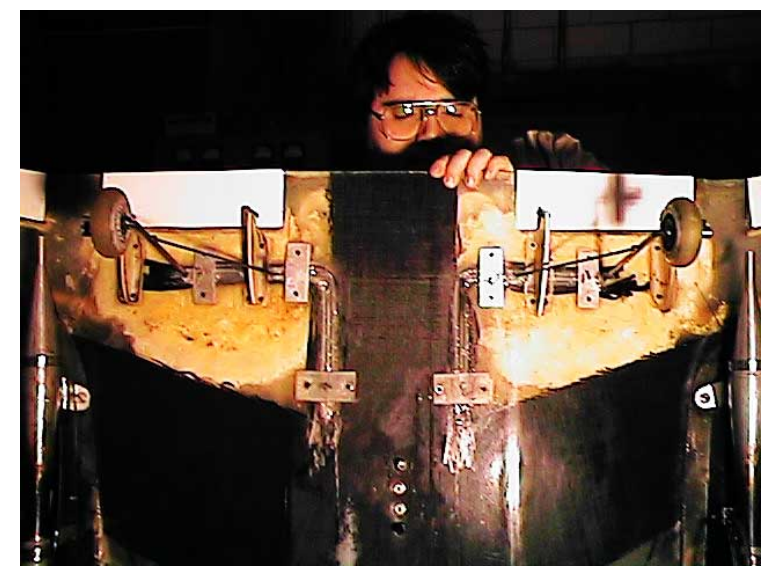

Figure 6.1.3 Main gear attachment

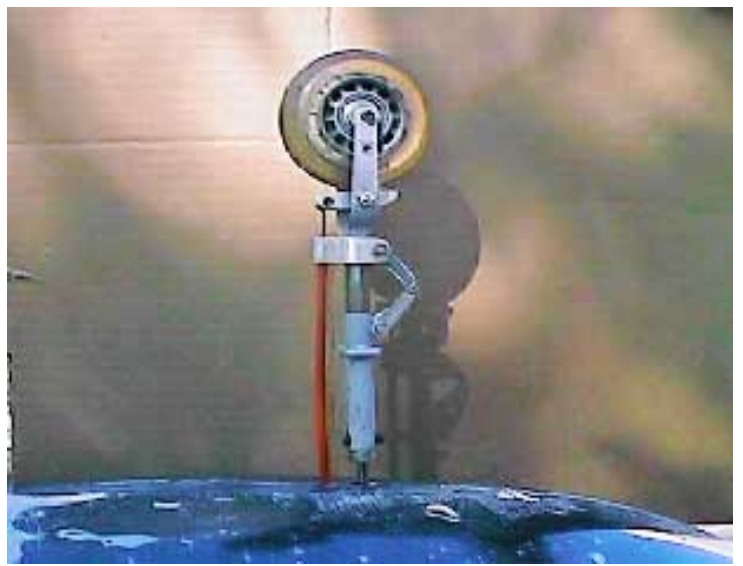

Figure 6.1.4 Nose gear strut

The main aircraft gear was comprised of $1 / 4$ inch steel "piano wire" with a supporting bar welded across the 90-degree bend. A molded channel was added to the bottom of the wing surface to house the metal leg. For increased takeoff speed, roller blade wheels were necessary to decrease rolling resistance and improve handling characteristics on the ground. Foam and rubber tires, commonly used with model airplanes, were not able to handle the wear and tear of the rough runway. For high-speed taxi tests, the landing gear proved to be sufficient to produce the necessary ground speeds and accelerations required for initial flight tests. Minor vibrations during initial startup were visibly seen, but after a few seconds, the visible vibrations damped quickly with an increased ground speed. The actual attachment of the main gear was immersed in silicon to provide an additional 
damping effect, which was then locked down with the fiberglass and aluminum straps. Some fiberglass straps were replaced with aluminum because of access wearing on the forward bolt attachments. The front nose gear, shown in Figure 6.1.4, was a commercially available model aircraft strut that used shock-absorbing springs. Initially, a braking system was employed on the nose gear, but it proved to be ineffective, causing difficulties after touchdown of the aircraft. For later flights, the brake system was removed and the B777 was allowed to coast on touchdown and stop naturally. Throughout preliminary tests, the main gear proved to be very reliable and robust to substantial structural loads. Directional handling of the nose gear was also satisfactory during the runway taxi testing. Figures 6.1.5 and 6.1.6 show video captured photographs of the aircraft passing in front of the pilot's position on the runway and returning to base.

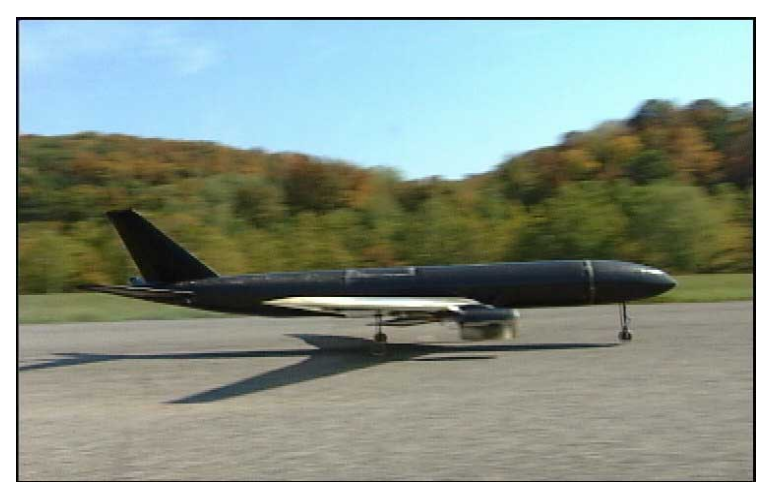

Figure 6.1.5 High speed taxi test

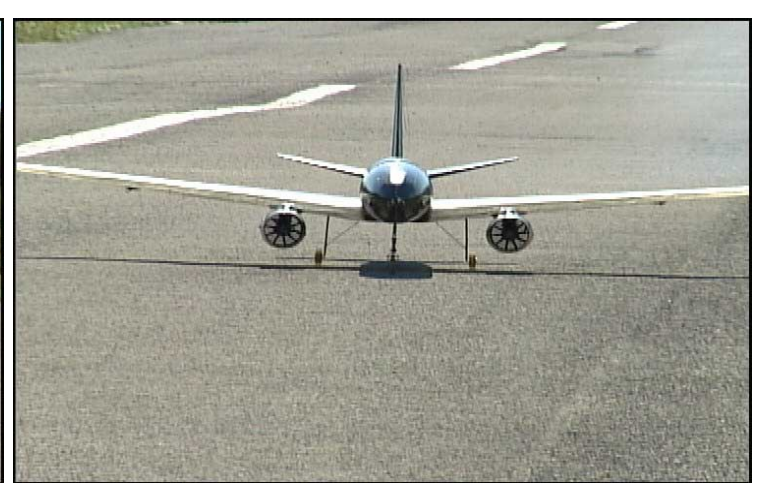

Figure 6.1.6 Return from taxi test

Overall, several runway speed tests were conducted and the pilot reported a quick response from both the throttle and nose wheel controls. The nose wheel servo was found to be over responsive, but was corrected with the R/C transmitter computer system using the dual rate capability. Ground testing showed that the range of the $\mathrm{R} / \mathrm{C}$ radio system exceeded the pilot's visual distance of the aircraft and easily met the minimum 150-foot range test with the antenna fully retracted; a standard test for R/C radio equipment. Once an $\mathrm{R} / \mathrm{C}$ aircraft has reached an altitude of approximately six feet, the ground effect on the radio receiver is reduced and the range is significantly extended. Initial range problems were detected with the on-board receiver batteries due to a power drain from the servos, reducing the voltage to the receiver. To overcome this, separate battery packs were used for the on-board receiver and servos. 


\section{Flight Data Collection}

For the estimation of the stability and control derivatives, control surface doublets were provided as input maneuvers during each flight test. The longitudinal doublets included elevator inputs, while the lateral-directional inputs included individual or combination maneuvers of aileron and rudder doublet pulses. The magnitude and excitation of the aircraft's dynamics played a role in determining the stability derivatives. As there was no on-board camera or visual feedback system for the pilot, practicing and implementing the PID maneuvers was a difficult task during the first set of instrumentation flights. For example, if the resulting pilot input amplitude was too low, there would not be enough excitation. If the input was to high, the aircraft could enter non-linear kinematics and aerodynamic conditions ${ }^{14}$. Initially some aircraft maneuvers recorded were found to exceed some of the sensor's maximum design ranges. Without feedback information available to the pilot, this was a learning process between pilot inputs and post flight analysis. Although the airfield provided a secluded area, flight test maneuvers had to be performed within a certain flight pattern. Figure 6.1.7 shows a diagram of the airfield area and flight pattern used for testing.

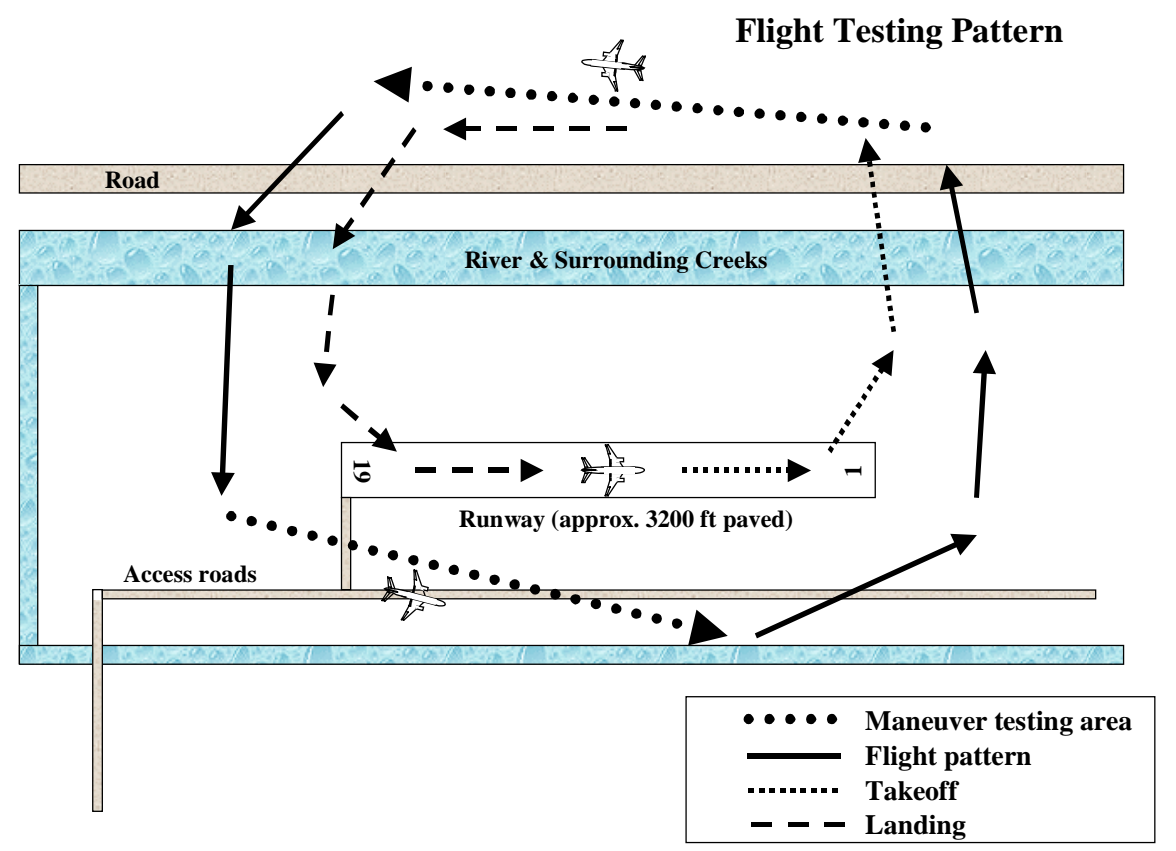

Figure 6.1.7 Flight pattern for the B777 flight testing activities 
Early flights with the electronic payload began to show a need for improving the execution of maneuvers and output data quality. The pilot needed to attempt to execute each flight test maneuver starting from a trimmed, wings-level condition. After the completion of each test flight, the data was downloaded and stored for post processing within a Matlab software environment. This environment allowed for easy conversion of vehicle parameters to their respective engineering units. Flight information was then passed onto a Unix based Sun station for analysis with the Fortran based "pEst" parameter estimation software. GetData ${ }^{34}$, a utility program for manipulating time history files, provided the ability to cut individual flight maneuver windows for PID analysis. Other GetData features included file format conversions, data manipulation, and data compression.

The longitudinal and lateral-directional surface deflection definitions for the elevator, aileron , and rudder are represented as:

$$
\begin{gathered}
\delta_{\mathrm{e}}=\frac{1}{2}\left(\delta_{\mathrm{e}_{1}}+\delta_{\mathrm{e}_{\mathrm{r}}}\right) \\
\delta_{a}=\frac{1}{2}\left(\delta_{a_{l}}+\delta_{a_{r}}\right) \\
\delta_{r}=\delta_{r}
\end{gathered}
$$

Figure 6.1.8 shows the orientation of the body axis forces and moments acting upon the aircraft.

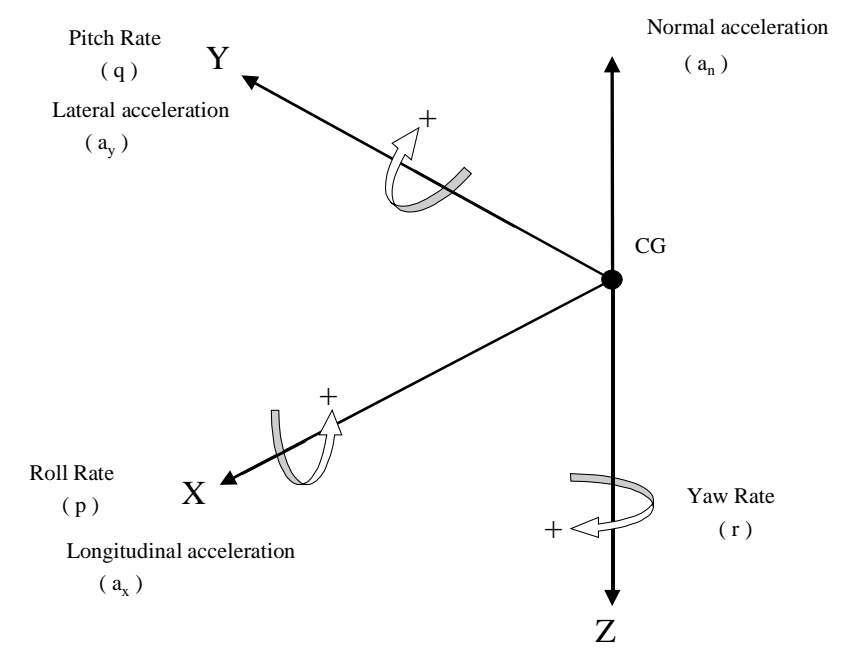

Figure 6.1.8 Body axis forces and moments acting on the aircraft 


\subsection{Flight Testing Maneuvers}

This section will discuss briefly objectives $2-4$, for Phase \#1 testing. Figure 6.2.1 below shows the first takeoff of the B777 model. Table 6.2.1 describes the aircraft configuration used for the first three test flights.

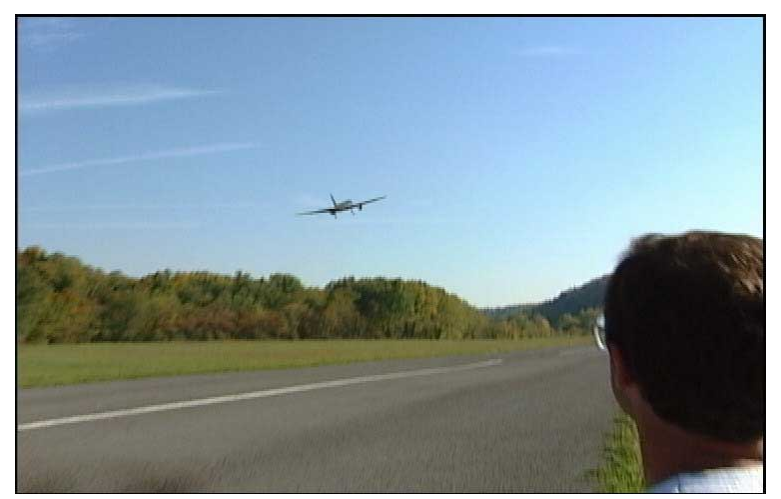

Figure 6.2.1 First flight of the B777 aircraft

Table 6.2.1 - Aircraft configuration for flights \#1, \#2, and \#3

\begin{tabular}{|l|l|}
\hline Takeoff weight & $33.8 \mathrm{lbs}$. \\
\hline Wing loading (approx.) & $47 \mathrm{oz} / \mathrm{ft}^{2}$ \\
\hline CG location & $26 \% \mathrm{MAC}$ \\
\hline
\end{tabular}

After the first flight, the pilot reported that the aircraft required a full down elevator trim, full right aileron trim, and approximately $1 / 3$ throttle to maintain a level cruise condition. The aircraft showed a substantial positive pitch rotation when inducing a high throttle setting, caused during low airspeeds. However, the pitching problem was reduced at higher airspeeds due to increased elevator effectiveness. The pilot reported small amounts of adverse yawing and a very good roll response from the ailerons (set at the high rates). A common practice after each flight was to have a quick debrief of the aircraft overall performance and handling qualities. From these debriefs, the crew was able to make any adjustments necessary for the pilot to become more comfortable with the aircraft at the end of the first set of flight tests. Handling qualities were reported as desirable with only minor pilot compensation for both longitudinal and lateral directional dynamics. This was considered to be quite an accomplishment given the fairly large size and weight of this R/C model. During the first two flights, the pilot performed several high altitude passes to test "stall" characteristics. From a propulsion point of view, the 
aircraft accelerated very well and exhibited excellent climbing performance. Initially, the elevator high rate setting was very responsive and difficult to control on the first two flights; however, there was not enough pitch response at the low rate setting. During the first two flights, the pilot advised us that the B777 was difficult to view with the naturally unpainted black carbon fiber. For this reason, a new paint scheme was adopted to improve the pilot's perception of the aircraft's orientation. This paint scheme, shown in Figure 6.2.2, featured an all white fuselage with only the top section of the wing painted.

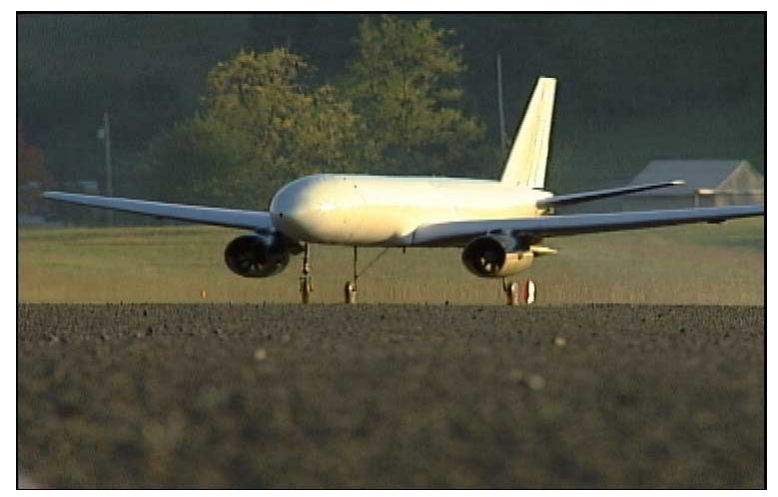

Figure 6.2.2 High visibility paint scheme

The bottom part of the wing with fiberglass and carbon fiber sections remained unpainted for a color contrast in flight. An additional advantage from the all white paint scheme was that it helped to reflect the heat from long exposures in the sun.

For the initial set of test flights, several weight configurations were chosen which were used to simulate the presence of the electronic payload. At this stage, the pilot used this artificial payload to gain flight experience at various weight configurations, without endangering the real electronic payload. In total, nine such flights were completed, which were either considered "empty weight" or a "dummy payload" configuration. Table 6.2.2 lists the weight configurations for Flights 1-9 using the artificial payload weight. 


\section{Table 6.2.2 - Weight configurations used for preliminary flight tests}

\begin{tabular}{|c|c|c|}
\hline Flight \# & A/C weight & Flaps \\
\hline 1 & $33.8 \mathrm{lb}$. & none \\
\hline 2 & $33.8 \mathrm{lb}$. & none \\
\hline 3 & $33.8 \mathrm{lb}$. & none \\
\hline 4 & $33.8 \mathrm{lb}$. & none \\
\hline 5 & $34.5 \mathrm{lb}$. & $\sqrt{ }$ \\
\hline 6 & $37.62 \mathrm{lb}$. & $\sqrt{ }$ \\
\hline 7 & $41.82 \mathrm{lb}$. & $\sqrt{ }$ \\
\hline 8 & $46.02 \mathrm{lb}$. & $\sqrt{ }$ \\
\hline 9 & $50.02 \mathrm{lb}$. & $\sqrt{ }$ \\
\hline
\end{tabular}

This slow incremental weight gain gave the pilot a chance to evaluate the model's performance and handling qualities. In Flights 1-4, the pilot did encounter some difficulties in slowing the vehicle airspeed for the landing approach. Installation of an inboard and outboard flaps system was found necessary to facilitate a slower landing approach. From Flight 5 and beyond, the installation of a flap system improved the aircraft handling during the landing approach. Deployment of the flaps performed as expected, with only minor corrections necessary for trimming the aircraft. The aircraft takeoff performance and in-flight handling was not substantially affected by the introduction of the "dummy" payload. Flights 5-9 included the addition of incremental weights to simulate the addition of the instrumentation. These early flights featured weight increases of approximately $4 \mathrm{lb}$. increments, and in all, a total of approximately 16 lbs. was introduced as dummy payload. This final payload value of $50 \mathrm{lbs}$. surpassed the necessary design requirement and insured a safe vehicle test bed for carrying the instrumentation payload. During Flight 9, the pilot reported a change in the aircraft handling qualities when the maximum payload weight was attempted. The aircraft still had acceptable flying qualities, but required a large piloting effort as expected due to the substantial weight increase. The planned instrumentation payload weight was targeted to be approximately $12.5 \mathrm{lb}$. Flight 9 showed the aircraft was capable of carrying an additional 4 pounds of payload. If needed, the additional payload could come in the form of increased fuel or battery power if necessary to extend the overall flight time. However, the pilot did note, that during Flight 9 it was necessary to use a higher power 
setting to prevent stalling in the turns and during the landing approach. In general, the dynamic characteristics were reported to be adequate for a model of this weight and size.

Prior to every flight of the B777, a routine startup and preparation procedure was completed for the aircraft and propulsion system. In terms of aircraft components, each control surface and major R/C components were inspected and evaluated for "flight worthiness". With respect to the engines, the throttle responses had to be constantly "retuned" to remain in a consistent RPM range for each flight test performed. Three specific RPM ranges were observed before every flight test, the idle, mid, and full throttle settings. Adjustments were necessary because of the varying weather conditions at the airfield. If this RPM imbalance was not adjusted prior to flight, the outcome typically resulted in an adverse yaw effect coming from the engines during testing.

For discussion purposes within this document, the B777 flights were divided into longitudinal and lateral-directional testing. Flights 10-18 refer to the longitudinal maneuvers, while Flights A-E refer to lateral-directional maneuvers. Before every flight, the aircraft was weighed and properly balanced; Table 6.2 .3 shows a listing of the weight configurations for each of the flight tests

Table 6.2.3 - Weight configurations used for payload flight tests

\begin{tabular}{|c|c|c|}
\hline Flight \# & A/C weight & Flaps \\
\hline 10 & $46.32 \mathrm{lb}$. & $\sqrt{ }$ \\
\hline 11 & $45.92 \mathrm{lb}$. & $\sqrt{ }$ \\
\hline 12 & $46.32 \mathrm{lb}$. & $\sqrt{ }$ \\
\hline 13 & $46.22 \mathrm{lb}$. & $\sqrt{ }$ \\
\hline 14 & $46.42 \mathrm{lb}$. & $\sqrt{ }$ \\
\hline 15 & $46.82 \mathrm{lb}$. & $\sqrt{ }$ \\
\hline 16 & $46.82 \mathrm{lb}$. & $\sqrt{ }$ \\
\hline 17 & $46.82 \mathrm{lb}$. & $\sqrt{ }$ \\
\hline 18 & $46.42 \mathrm{lb}$. & $\sqrt{ }$ \\
\hline A & $46.42 \mathrm{lb}$. & $\sqrt{ }$ \\
\hline B & $46.42 \mathrm{lb}$. & $\sqrt{ }$ \\
\hline C & $46.60 \mathrm{lb}$. & $\sqrt{ }$ \\
\hline D & $46.60 \mathrm{lb}$. & $\sqrt{ }$ \\
\hline E & $47.12 \mathrm{lb}$. & $\sqrt{ }$ \\
\hline
\end{tabular}


The "slightly varying" weight values listed in Table 6.2.3 were due to regular aircraft maintenance and repairs. Flights 10-14 were considered as the initial payload test flights, which allowed the assessment of the performance of the components of the payload. Overall aircraft flight operations were successful, with only minor difficulties with the pitch rate sensor. In Flight 13, the pitch rate sensor failed for that specific flight, however lab tests prior to flight gave no indication of a problem. From Flight 14-18, all three gyros were positioned to record the aircraft's angular pitch rate, so to ensure measurements during longitudinal maneuvers. During Flights A-E, lateral-directional testing, the failed gyro was replaced so that all three angular rates, pitch, roll and yaw could be recorded.

Standard doublets were performed on each major control surface. For longitudinal testing elevator doublets were completed. For lateral-direction testing, aileron and rudder doublets were completed individually and as a combination lateral maneuver. A main objective for each flight was to maximize the effort to complete as many maneuvers as possible within the allotted flight time. Within that time frame, an average of nine to twelve maneuvers were collected per test flight. Table 6.2.4 shows the overall number of maneuvers collected.

Table 6.2.4 - Overall number of maneuvers performed during actual flight tests

\begin{tabular}{|c|c|c|c|c|}
\hline Flight \# & $\delta_{\mathrm{e}}$ & $\delta_{\mathrm{a}}$ & $\delta_{\mathrm{r}}$ & $\delta_{\mathrm{a}} / \delta_{\mathrm{r}}$ Combo \\
\hline 10 & 2 & $\mathrm{n} / \mathrm{a}$ & $\mathrm{n} / \mathrm{a}$ & $\mathrm{n} / \mathrm{a}$ \\
\hline 11 & 4 & $\mathrm{n} / \mathrm{a}$ & $\mathrm{n} / \mathrm{a}$ & $\mathrm{n} / \mathrm{a}$ \\
\hline 12 & 0 & $\mathrm{n} / \mathrm{a}$ & $\mathrm{n} / \mathrm{a}$ & $\mathrm{n} / \mathrm{a}$ \\
\hline 13 & 4 & $\mathrm{n} / \mathrm{a}$ & $\mathrm{n} / \mathrm{a}$ & $\mathrm{n} / \mathrm{a}$ \\
\hline 14 & 0 & $\mathrm{n} / \mathrm{a}$ & $\mathrm{n} / \mathrm{a}$ & $\mathrm{n} / \mathrm{a}$ \\
\hline 15 & 5 & $\mathrm{n} / \mathrm{a}$ & $\mathrm{n} / \mathrm{a}$ & $\mathrm{n} / \mathrm{a}$ \\
\hline 16 & 7 & $\mathrm{n} / \mathrm{a}$ & $\mathrm{n} / \mathrm{a}$ & $\mathrm{n} / \mathrm{a}$ \\
\hline 17 & 8 & $\mathrm{n} / \mathrm{a}$ & $\mathrm{n} / \mathrm{a}$ & $\mathrm{n} / \mathrm{a}$ \\
\hline 18 & 10 & $\mathrm{n} / \mathrm{a}$ & $\mathrm{n} / \mathrm{a}$ & $\mathrm{n} / \mathrm{a}$ \\
\hline $\mathrm{A}$ & 0 & 0 & 0 & 10 \\
\hline B & 0 & 0 & 0 & 13 \\
\hline C & 0 & 0 & 0 & 12 \\
\hline D & 1 & 2 & 2 & 1 \\
\hline E & 0 & 4 & 4 & $\mathbf{3 8}$ \\
\hline TOTAL & $\mathbf{4 0}$ & $\mathbf{6}$ & $\mathbf{6}$ & $\mathrm{A}$ \\
\hline
\end{tabular}

Note: All numbered flights $(12,13$, etc.) were for longitudinal maneuvers only. All letter flights (A, B, etc.) focused on completing lateral-directional maneuvers. 
The choice of parameters to extract from usable flight data depended upon which control surfaces inputs were given to the model during a flight test maneuver. For the longitudinal components, the normal force $\left(\mathrm{C}_{\text {Norm }}\right)$, pitching moment $\left(\mathrm{C}_{\mathrm{m}}\right)$, and axial force $\left(\mathrm{C}_{\mathrm{A}}\right)$ were estimated. For estimation purposes, the active states for the longitudinal case included $\alpha$, $\mathrm{q}$ with the active responses $\alpha, \mathrm{q}, \mathrm{a}_{\mathrm{n}}$, and $\mathrm{a}_{\mathrm{x}}$. For the lateral-directional components, the lateral force $\left(C_{y}\right)$, rolling moments $\left(C_{1}\right)$, and yawing moments $\left(C_{n}\right)$ were estimated. For estimation purposes, the active states for the lateral case included $\beta, \mathrm{p}$, and $\mathrm{r}$, while the active responses included: $\beta, \mathrm{p}, \mathrm{r}$, and $\mathrm{a}_{\mathrm{y}}$. Tables 6.2.5 and 6.2.6 show the longitudinal and lateral-directional coefficients estimated for each style of maneuver input.

Table 6.2.5 - Longitudinal coefficient table listing for flight test analysis

\begin{tabular}{|c|c|c|c|c|}
\hline Derivative & $\delta_{\mathrm{e}}$ & $\delta_{\mathrm{a}}$ & $\delta_{\mathrm{r}}$ & $\delta_{\mathrm{a}} / \delta_{\mathrm{r}}$ Combo \\
\hline $\mathrm{C}_{\mathrm{Norm}_{\mathrm{O}}}$ & $\sqrt{ }$ & $\mathrm{X}$ & $\mathrm{X}$ & $\mathrm{X}$ \\
\hline $\mathrm{C}_{\mathrm{Norm}_{\alpha}}$ & $\sqrt{ }$ & $X$ & $X$ & $\mathrm{X}$ \\
\hline $\mathrm{C}_{\mathrm{Norm}_{\mathrm{q}}}$ & $\sqrt{ }$ & $X$ & $X$ & $X$ \\
\hline $\mathrm{C}_{\mathrm{Norm}} \delta_{\mathrm{e}}$ & $\sqrt{ }$ & $X$ & $X$ & $\mathrm{X}$ \\
\hline $\mathrm{C}_{\mathrm{m}_{\mathrm{O}}}$ & $\sqrt{ }$ & $X$ & $X$ & $\mathrm{X}$ \\
\hline $\mathrm{C}_{\mathrm{m}_{\alpha}}$ & $\sqrt{ }$ & $X$ & $X$ & $X$ \\
\hline $\mathrm{C}_{\mathrm{m}_{\mathrm{q}}}$ & $\sqrt{ }$ & $\mathrm{X}$ & $\mathrm{X}$ & $\mathrm{X}$ \\
\hline $\mathrm{C}_{\mathrm{m}_{\delta_{\mathrm{e}}}}$ & $\sqrt{ }$ & $\mathrm{X}$ & $\mathrm{X}$ & $\mathrm{X}$ \\
\hline $\mathrm{C}_{\mathrm{A}_{\mathrm{O}}}$ & $\sqrt{ }$ & $X$ & $X$ & $X$ \\
\hline $\mathrm{C}_{\mathrm{A}_{\alpha}}$ & $\sqrt{ }$ & $X$ & X & $X$ \\
\hline $\mathrm{C}_{\mathrm{A}_{\mathrm{q}}}$ & $\sqrt{ }$ & $X$ & $X$ & $X$ \\
\hline $\mathrm{C}_{\mathrm{A}_{\delta_{\mathrm{e}}}}$ & $\sqrt{ }$ & $X$ & $X$ & $\mathrm{X}$ \\
\hline
\end{tabular}


Table 6.2.6 - Lateral-Directional coefficient table listing for flight test analysis

\begin{tabular}{|c|c|c|c|c|}
\hline Derivative & $\delta_{\mathrm{e}}$ & $\delta_{\mathrm{a}}$ & $\delta_{\mathrm{r}}$ & $\delta_{\mathrm{a}} / \delta_{\mathrm{r}}$ Combo \\
\hline $\mathrm{C}_{\mathrm{l}_{\mathrm{O}}}$ & $X$ & $\sqrt{ }$ & $\sqrt{ }$ & $\sqrt{ }$ \\
\hline $\mathrm{C}_{\mathrm{l}_{\beta}}$ & $X$ & $\sqrt{ }$ & $\sqrt{ }$ & $\sqrt{ }$ \\
\hline $\mathrm{C}_{\mathrm{l}_{\mathrm{p}}}$ & $X$ & $\sqrt{ }$ & $\sqrt{ }$ & $\sqrt{ }$ \\
\hline $\mathrm{C}_{\mathrm{l}_{\mathrm{r}}}$ & $X$ & $\sqrt{ }$ & $\sqrt{ }$ & $\sqrt{ }$ \\
\hline $\mathrm{C}_{\mathrm{l}_{\delta_{\mathrm{a}}}}$ & $X$ & $\sqrt{ }$ & $\mathrm{X}$ & $\sqrt{ }$ \\
\hline $\mathrm{C}_{{ }^{{ }^{\prime}}}$ & $X$ & $\mathrm{X}$ & $\sqrt{ }$ & $\sqrt{ }$ \\
\hline $\mathrm{C}_{\mathrm{n}_{\mathrm{o}}}$ & $X$ & $\sqrt{ }$ & $\sqrt{ }$ & $\sqrt{ }$ \\
\hline $\mathrm{C}_{\mathrm{n} \beta}$ & $X$ & $\sqrt{ }$ & $\sqrt{ }$ & $\sqrt{ }$ \\
\hline $\mathrm{C}_{\mathrm{n}_{\mathrm{p}}}$ & $\mathrm{X}$ & $\sqrt{ }$ & $\sqrt{ }$ & $\sqrt{ }$ \\
\hline $\mathrm{C}_{\mathrm{n}_{\mathrm{r}}}$ & $X$ & $\sqrt{ }$ & $\sqrt{ }$ & $\sqrt{ }$ \\
\hline $\mathrm{C}_{\mathrm{n}_{\delta_{\mathrm{a}}}}$ & $X$ & $\sqrt{ }$ & $X$ & $\sqrt{ }$ \\
\hline $\mathrm{C}_{\mathrm{n}_{\delta_{\mathrm{r}}}}$ & $X$ & $\mathrm{X}$ & $\sqrt{ }$ & $\sqrt{ }$ \\
\hline $\mathrm{C}_{\mathrm{y}_{\mathrm{o}}}$ & $X$ & $\sqrt{ }$ & $\sqrt{ }$ & $\sqrt{ }$ \\
\hline $\mathrm{C}_{\mathrm{y} \beta}$ & $X$ & $\sqrt{ }$ & $\sqrt{ }$ & $\sqrt{ }$ \\
\hline $\mathrm{C}_{\mathrm{y}_{\mathrm{p}}}$ & $X$ & $\sqrt{ }$ & $\sqrt{ }$ & $\sqrt{ }$ \\
\hline $\mathrm{C}_{\mathrm{y}_{\mathrm{r}}}$ & $X$ & $\sqrt{ }$ & $\sqrt{ }$ & $\sqrt{ }$ \\
\hline $\mathrm{C}_{\mathrm{y} \delta_{\mathrm{a}}}$ & $X$ & $\sqrt{ }$ & $\mathrm{X}$ & $\sqrt{ }$ \\
\hline $\mathrm{C}_{\mathrm{y}_{\delta_{\mathrm{r}}}}$ & $X$ & $\mathrm{X}$ & $\sqrt{ }$ & $\sqrt{ }$ \\
\hline
\end{tabular}

For both longitudinal and lateral-directional aerodynamic biases $\left(\mathrm{C}_{\mathrm{Norm}_{\mathrm{O}}}, \mathrm{C}_{\mathrm{m}_{\mathrm{O}}}, \mathrm{C}_{\mathrm{A}_{\mathrm{O}}}\right.$, $\mathrm{C}_{\mathrm{O}_{\mathrm{O}}}, \mathrm{C}_{\mathrm{n}_{\mathrm{O}}}$, and $\mathrm{C}_{\mathrm{y}_{\mathrm{O}}}$ ), these estimated values are expected to represent the overall forces and moments at zero (angle of attach / sideslip) angle with a zero (pitch / lateral) control surface deflection for either the longitudinal or lateral case ${ }^{10,28}$.

For the off-line batch estimation software, the response variable (such as angle of attack or pitch rate) has two features. First the response can either be considered active or non-active during a specific maneuver and second can have a specific weighting value 
within the estimation process ${ }^{33}$. When a response was active a variable time history value was computed. The weighting of a response specifies the value within the cost function. Altering weights can help improve the matching of the computed and measured responses, but should be carefully set so that the data matches are not too erratic. Adjustments were made to account for discrepancies in weight, temperature and atmospheric conditions. These values were then held constant for all maneuvers within that particular flight. For the B777 analysis the values were modified accordingly and shown in Table 6.2.7.

Table 6.2.7 - Weight settings for aircraft states (off-line analysis)

\begin{tabular}{|c|c|c|c|c|c|c|c|c|}
\hline Flight \# & $\alpha$ & $q$ & $a_{n}$ & $a_{x}$ & $\beta$ & $\mathrm{p}$ & $r$ & $\mathrm{a}_{\mathrm{y}}$ \\
\hline 10 & 5 & 8 & 35 & 100 & $\mathrm{n} / \mathrm{a}$ & $\mathrm{n} / \mathrm{a}$ & $\mathrm{n} / \mathrm{a}$ & $\mathrm{n} / \mathrm{a}$ \\
\hline 11 & 5 & 8 & 35 & 100 & $\mathrm{n} / \mathrm{a}$ & $\mathrm{n} / \mathrm{a}$ & $\mathrm{n} / \mathrm{a}$ & $\mathrm{n} / \mathrm{a}$ \\
\hline 12 & 5 & 8 & 35 & 100 & $\mathrm{n} / \mathrm{a}$ & $\mathrm{n} / \mathrm{a}$ & $\mathrm{n} / \mathrm{a}$ & $\mathrm{n} / \mathrm{a}$ \\
\hline 13 & 5 & 8 & 35 & 100 & $\mathrm{n} / \mathrm{a}$ & $\mathrm{n} / \mathrm{a}$ & $\mathrm{n} / \mathrm{a}$ & $\mathrm{n} / \mathrm{a}$ \\
\hline 14 & 5 & 8 & 35 & 100 & $\mathrm{n} / \mathrm{a}$ & $\mathrm{n} / \mathrm{a}$ & $\mathrm{n} / \mathrm{a}$ & $\mathrm{n} / \mathrm{a}$ \\
\hline 15 & 5 & 8 & 35 & 100 & $\mathrm{n} / \mathrm{a}$ & $\mathrm{n} / \mathrm{a}$ & $\mathrm{n} / \mathrm{a}$ & $\mathrm{n} / \mathrm{a}$ \\
\hline 16 & 5 & 8 & 35 & 100 & $\mathrm{n} / \mathrm{a}$ & $\mathrm{n} / \mathrm{a}$ & $\mathrm{n} / \mathrm{a}$ & $\mathrm{n} / \mathrm{a}$ \\
\hline 17 & 5 & 8 & 35 & 100 & $\mathrm{n} / \mathrm{a}$ & $\mathrm{n} / \mathrm{a}$ & $\mathrm{n} / \mathrm{a}$ & $\mathrm{n} / \mathrm{a}$ \\
\hline 18 & 5 & 8 & 35 & 100 & $\mathrm{n} / \mathrm{a}$ & $\mathrm{n} / \mathrm{a}$ & $\mathrm{n} / \mathrm{a}$ & $\mathrm{n} / \mathrm{a}$ \\
\hline A & $\mathrm{n} / \mathrm{a}$ & $\mathrm{n} / \mathrm{a}$ & $\mathrm{n} / \mathrm{a}$ & $\mathrm{n} / \mathrm{a}$ & 3 & 0.7 & 1 & 30 \\
\hline $\mathrm{B}$ & $\mathrm{n} / \mathrm{a}$ & $\mathrm{n} / \mathrm{a}$ & $\mathrm{n} / \mathrm{a}$ & $\mathrm{n} / \mathrm{a}$ & $\mathrm{n} / \mathrm{a}$ & 1.8 & 1.2 & 100 \\
\hline $\mathrm{C}$ & $\mathrm{n} / \mathrm{a}$ & $\mathrm{n} / \mathrm{a}$ & $\mathrm{n} / \mathrm{a}$ & $\mathrm{n} / \mathrm{a}$ & 1 & 1 & 1 & 20 \\
\hline $\mathrm{D}$ & $\mathrm{n} / \mathrm{a}$ & $\mathrm{n} / \mathrm{a}$ & $\mathrm{n} / \mathrm{a}$ & $\mathrm{n} / \mathrm{a}$ & 2.5 & 1 & 1 & 100 \\
\hline $\mathrm{E}$ & $\mathrm{n} / \mathrm{a}$ & $\mathrm{n} / \mathrm{a}$ & $\mathrm{n} / \mathrm{a}$ & $\mathrm{n} / \mathrm{a}$ & 2.5 & 1 & 1 & 100 \\
\hline
\end{tabular}

For the next two sections, the off-line estimation results obtained using the pEst software are shown for both the longitudinal and lateral-directional coefficients. All figures corresponding to sections 6.3 and 6.4 were placed at the end of the section. 


\subsection{Longitudinal Results}

Figures 6.3.1-6.3.29 represent the results for the longitudinal stability and control derivatives of the B777 presented in terms of estimates of aerodynamic coefficients as well as the randomly selected time history comparisons between the measured and computed aircraft responses. Estimates were obtained for the normal force, pitching moment, and axial force $\left(\mathrm{C}_{\text {Norm }}, \mathrm{C}_{\mathrm{m}}\right.$, and $\left.\mathrm{C}_{\mathrm{A}}\right)$. The "Norm" subscript was used to make it easier to distinguish between $\mathrm{C}_{\mathrm{n}}$ (yaw moment) for the lateral directional case. Each estimate is presented with a corresponding Cramer-Rao bound represented as a vertical bar. The data points are categorized by flight, so it can be seen which flights produced that particular longitudinal maneuver. Each of the figures discussed are located at the end of this section.

Figures 6.3.1-6.3.3 show a sample time history of the aircraft parameters for longitudinal Flight 17 from takeoff to landing, including flight information for: $\alpha$, pitch rate, normal acceleration, axial acceleration, velocity, dynamic pressure, and altitude, along with corresponding control surface deflections $\left(\delta_{\mathrm{e}}, \delta_{\mathrm{a}}, \delta_{\mathrm{r}}\right)$. The beta channel was not included with the initial longitudinal flights and was left as an open channel for later use in lateral-directional testing. Typical ranges for the pitch rate were \pm 20 to \pm 40 $\mathrm{deg} / \mathrm{sec}$ depending on the maneuver, normal acceleration ranged from -1 to a $4 \mathrm{~g}$ maximum, and airspeed values ranged between 80 to $120 \mathrm{ft} / \mathrm{sec}$ during flight test maneuvers. Figure 6.3.3 shows the time history of each of the major control surfaces from Flight 17. Prior to the launching of a flight test, the engines were warmed up and running as each team member reached their assigned duty positions. After all team members achieved their starting positions, the computer system was then activated. Within the first 100 seconds you can see two large pulses recorded before the aircraft was launched. As a common practice, the pilot would execute a radio range test of one or two pulses to insure the transmitter was functioning correctly.

Figures 6.3.4-6.3.13 shows sample comparisons of measured and computed time histories collected from each of the longitudinal test flights, specifically:

- Flight 11, Maneuver 3

(Figures 6.3.4-6.3.5)

- Flight 15, Maneuver 3

(Figures 6.3.6-6.3.7)

- Flight 16, Maneuver 6

(Figures 6.3.8-6.3.9) 
- Flight 17, Maneuver 3

- Flight 18, Maneuver 9
(Figures 6.3.10-6.3.11)

(Figures 6.3.12-6.3.13)

For example, the outputs from the estimation process show the measured and computed responses for the $\alpha$, pitch rate, normal acceleration, and axial acceleration responses in Figure 6.3.10. The control input $\delta_{\mathrm{e}}$ for the longitudinal case, velocity, dynamic pressure, and altitude are shown in the accompanying secondary set of plots, as in Figure 6.3.11. Maneuver responses shown for the longitudinal flights were chosen randomly, with one maneuver selected from each of the test flights. Overall, the angle of attack, pitch rate, and normal acceleration gave reasonable matches for each of the maneuver periods shown. As expected, the axial acceleration values were very noisy with respect to other parameters. The $a_{x}$ measurements were considered to be reasonably matching several peaks in some of the maneuvers. For this analysis, filtered data was tested, in an attempt to improve the axial acceleration computed responses. However, there were very minor changes, with respect to the estimated values, using a set of additional filtered signals. It was noticed that when a filter signal was used, problems of phase shifting were noticed in the output responses. Trying various filtering techniques brings up a concern of losing aircraft dynamic information and becomes an issue when analyzing and comparing various sets of flight data. Using the data acquired directly from the onboard signals or filtering data was found to have a minor effect on the response matches. For future work the actual onboard pre-filtered signals would be used during testing. Filtered data did decrease computational time, but when overlaid against onboard data there was a very small difference found in response matches; as well as actual estimated values. This shows the robustness and capabilities of the estimation software.

Figures 6.3.14-6.3.29 show the off-line estimation results plotted against the average maneuver velocity and average angle of attack. This was done to give two perspectives of the flight data. Since no visual cue was available for the pilot, it was difficult to hold any particular attitude for each test maneuver of the aircraft. Visually from the ground, the pilot attempted to try and maintain the aircraft at a straight and level position before attempting any test maneuver. However, due to problems of depth perception, from the pilot's angle, actual airspeed and angle of attack, along with sometimes-difficult atmospheric conditions a straight and level starting position was not 
always possible. This difficulty resulted in seeing "groupings" of certain sets of flight data for the various estimates obtained. For the sake of discussion, all estimates were plotted versus the aircraft velocity and angle of attack.

Figures 6.3.14-6.3.15 represent the normal force and pitch moment of the aerodynamic bias. These values are typically found to be zero, but this information could be indicative of the effect of varying airspeeds, along with windy flight conditions. The "grouping" seems to remain fairly constant throughout the flights. The pitching moment did bounce positively and negatively around zero, showing a greater effect of varying flight conditions particularly in early flights.

Figures 6.3.16- 6.3.17 represent the normal force and pitch moment estimates due to the angle of attack $\left(\mathrm{C}_{\mathrm{Norm}_{\alpha}}, \mathrm{C}_{\mathrm{m}_{\alpha}}\right)$. There was a consistency overlaying the values between the Cramer-Rao bounds. The values for the normal force and pitch moments seemed not to be affected with an increase in airspeed. The $\mathrm{C}_{\mathrm{m}_{\alpha}}$ value showed longitudinal static stability throughout the maneuvers. Figures 6.3.18-6.3.19 represent the normal force and pitch moment estimates due to the pitch rate $\left(\mathrm{C}_{\mathrm{Norm}_{\mathrm{q}}}, \mathrm{C}_{\mathrm{m}_{\mathrm{q}}}\right)$. Over the entire velocity range, no distinctive pattern emerged from these estimates. The actual estimates were found to be higher in value than normally expected. Figures 6.3.20-6.3.21 represent the normal force and pitch moment effectiveness of the elevator $\left(\mathrm{C}_{\mathrm{Norm}_{\delta_{\mathrm{e}}}}, \mathrm{C}_{\mathrm{m}_{\delta_{\mathrm{e}}}}\right)$. The normal force coefficient tends to increase with velocity, but decrease with respect to angle of attack. The pitching moment values remained fairly constant over all airspeed ranges.

Figure 6.3.22-6.3.23 represent the axial force for the aerodynamic bias and the axial force due to angle of attack $\left(\mathrm{C}_{\mathrm{A}_{\mathrm{O}}}, \mathrm{C}_{\mathrm{A}_{\alpha}}\right)$. For earlier flights 11-13, the axial aerodynamic bias was difficult to determine. The Cramer-Rao bound values were larger than other flights; which could be attributed to the difficultly in matching the measured and computed $a_{x}$ response, as seen in figure 6.3.4. The overall $a_{x}$ match for earlier flights was poor when compared with later flight maneuvers. This could be due to interference issues encountered early on in the program (as discussed in the previous section). Figures 6.3.24-6.3.25 represent the axial force due to the pitching moment and elevator 
$\left(\mathrm{C}_{\mathrm{A}_{\mathrm{q}}}, \mathrm{C}_{\mathrm{A}_{\delta_{\mathrm{e}}}}\right)$. Clearly the pitch rate and elevator effectiveness increased as the velocity increased, which was not apparent when viewing the data versus angle of attack.

Figures 6.3.26-6.3.29 represent the computational values for $\mathrm{C}_{\mathrm{Z}_{\mathrm{O}}}, \mathrm{C}_{\mathrm{Z}_{\alpha}}, \mathrm{C}_{\mathrm{Z}_{\mathrm{q}}}$, and $\mathrm{C}_{\mathrm{Z}_{\delta_{\mathrm{e}}}}$. These values were not generated by the estimation process and do not have a representative $\mathrm{CRB}$ shown for the estimate. The following formula shows the transformation, where $\mathrm{x}$ is the estimate in question (angle of attack, pitch rate, etc.) and where $\alpha$ from average maneuver was used:

$$
\mathrm{C}_{\mathrm{Z}_{\mathrm{X}}}=\mathrm{C}_{\mathrm{A}_{\mathrm{X}}} \sin (\alpha)-\mathrm{C}_{\mathrm{Norm}_{\mathrm{X}}} \cos (\alpha)
$$

These values were necessary for use within the state-space variable modeling phase in Chapter 7. 
Flight 17, Full Flight Time History
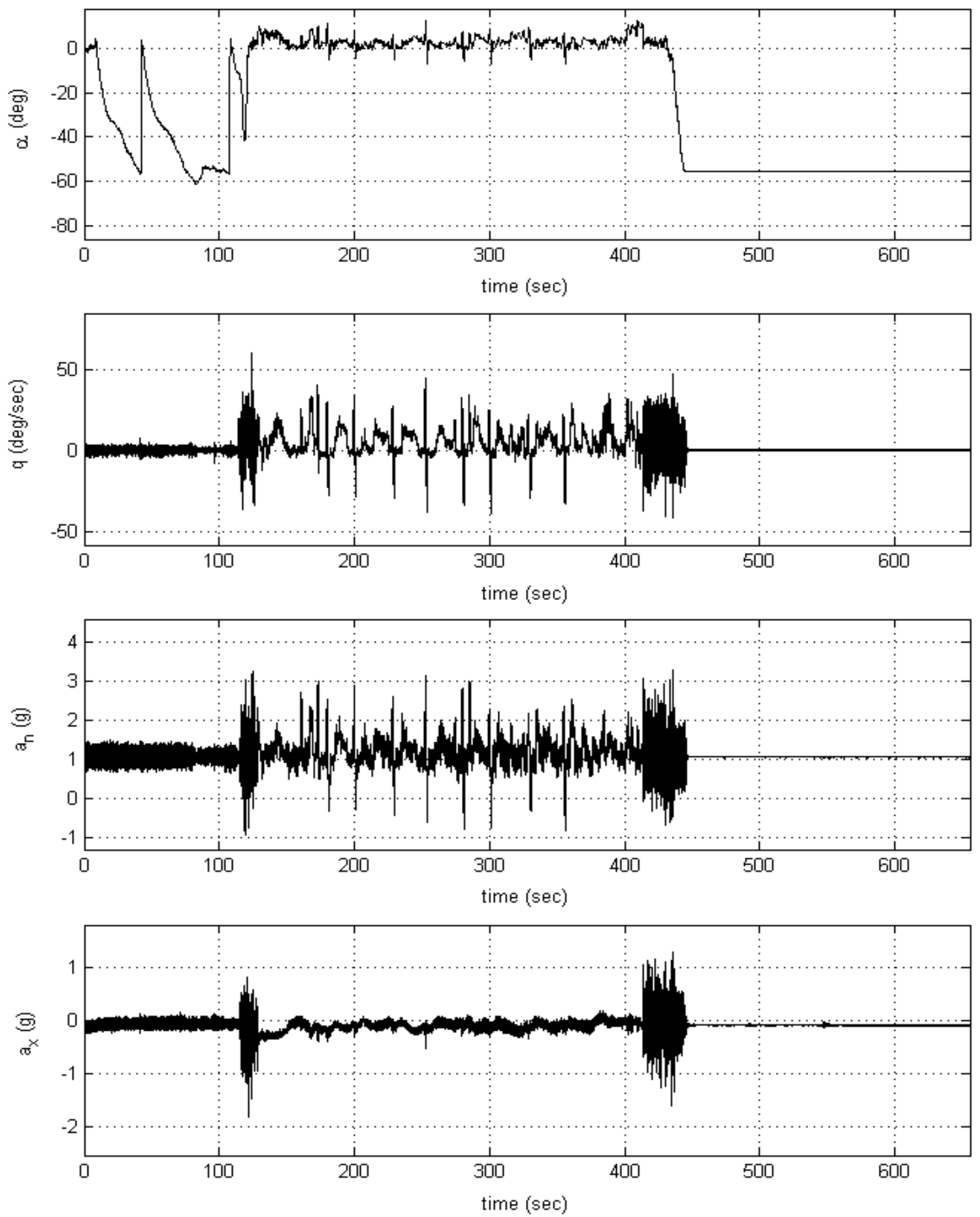

Figure 6.3.1 Sample time history of aircraft parameters for longitudinal Flight 17 ( $\alpha$, pitch rate, normal acceleration, axial acceleration) 
Flight 17, Full Flight Time History
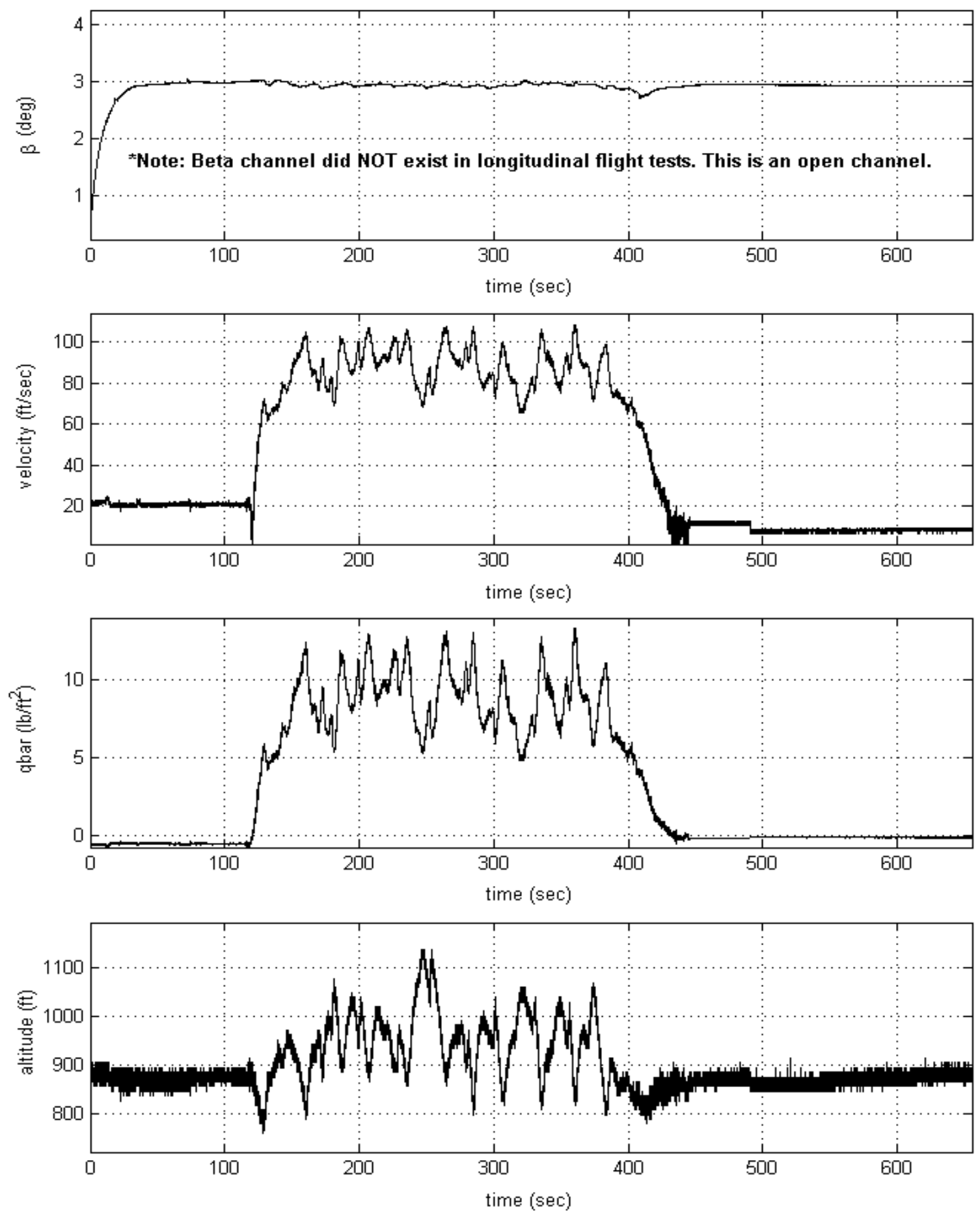

Figure 6.3.2 Sample time history of aircraft parameters for longitudinal Flight 17 ( $\beta$, velocity, dynamic pressure, altitude) 
Flight 17, Full Flight Time History
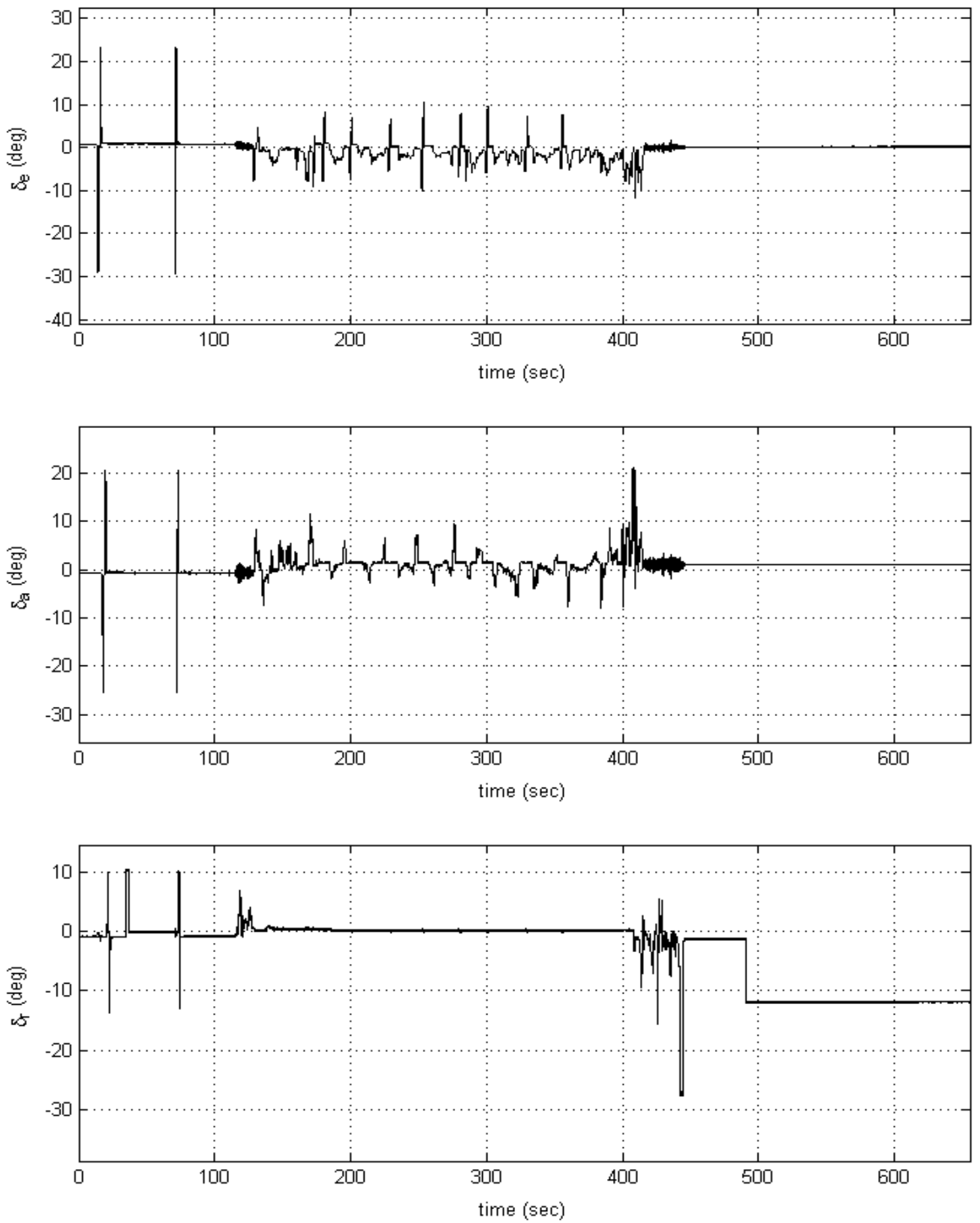

Figure 6.3.3 Sample time history of aircraft parameters for longitudinal Flight 17 $\left(\delta_{\mathrm{e}}, \delta_{\mathrm{a}}, \delta_{\mathrm{r}}\right)$. 
Flight 11, Maneuver 3 (longitudinal input)
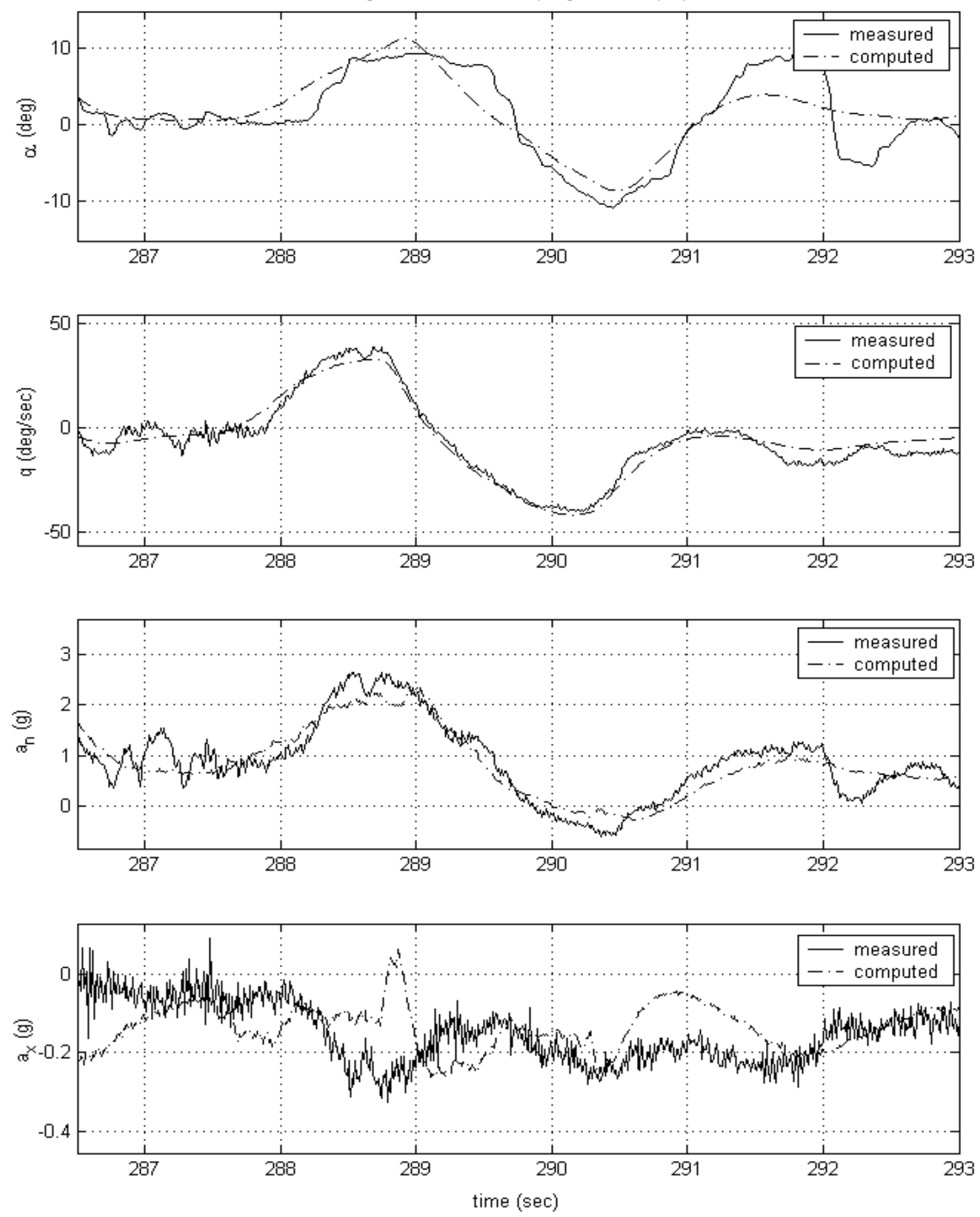

Figure 6.3.4 Comparison of measured and computed time histories from Flight 11, Maneuver 3, longitudinal input ( $\alpha$, pitch rate, normal acceleration, axial acceleration) 
Flight 11, Maneuver 3 (longitudinal input)
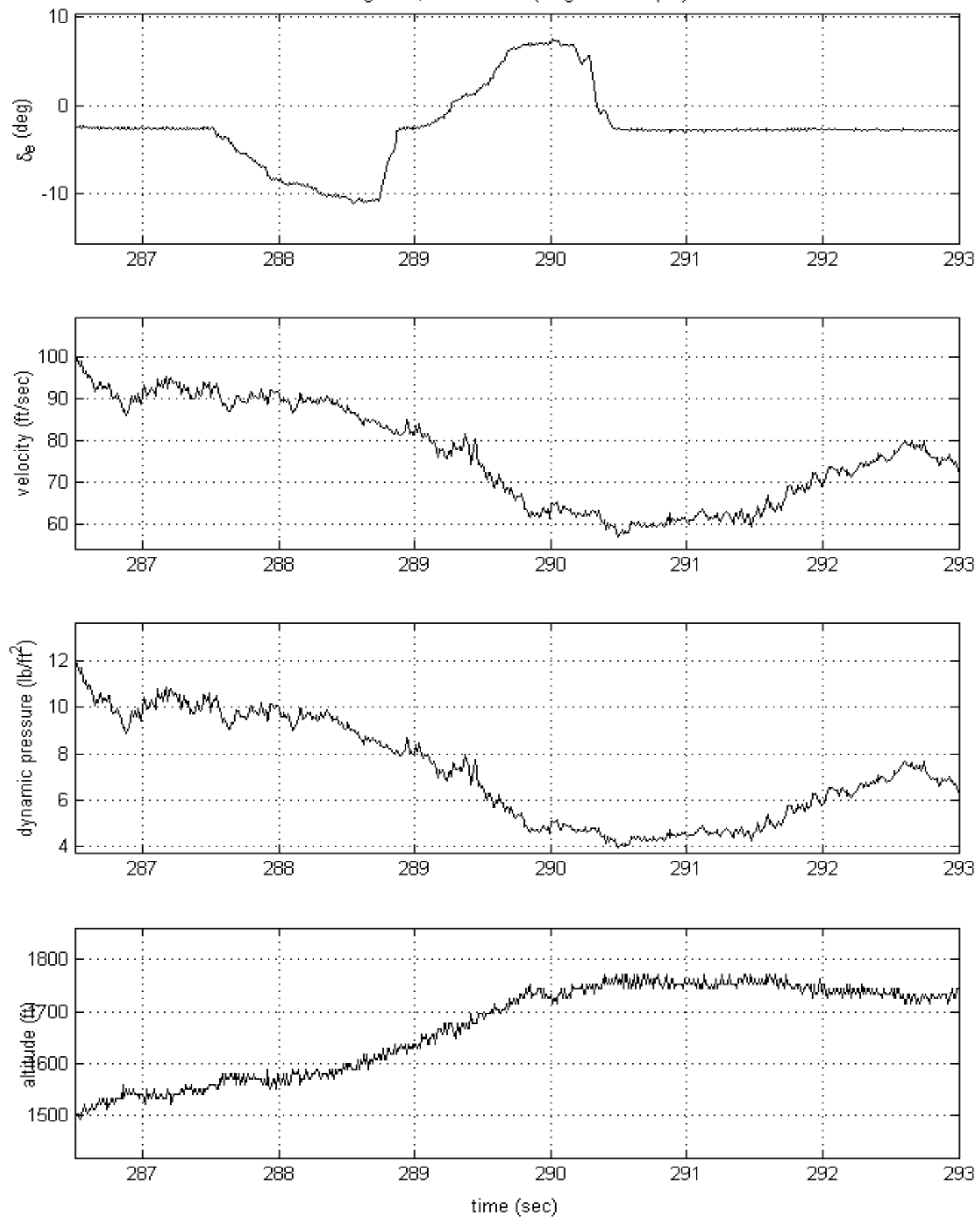

Figure 6.3.5 Comparison of measured and computed time histories from Flight 11, Maneuver 3, longitudinal input ( $\delta_{\mathrm{e}}$, velocity, dynamic pressure, altitude) 
Flight 15, Maneuver 3 (longitudinal input)
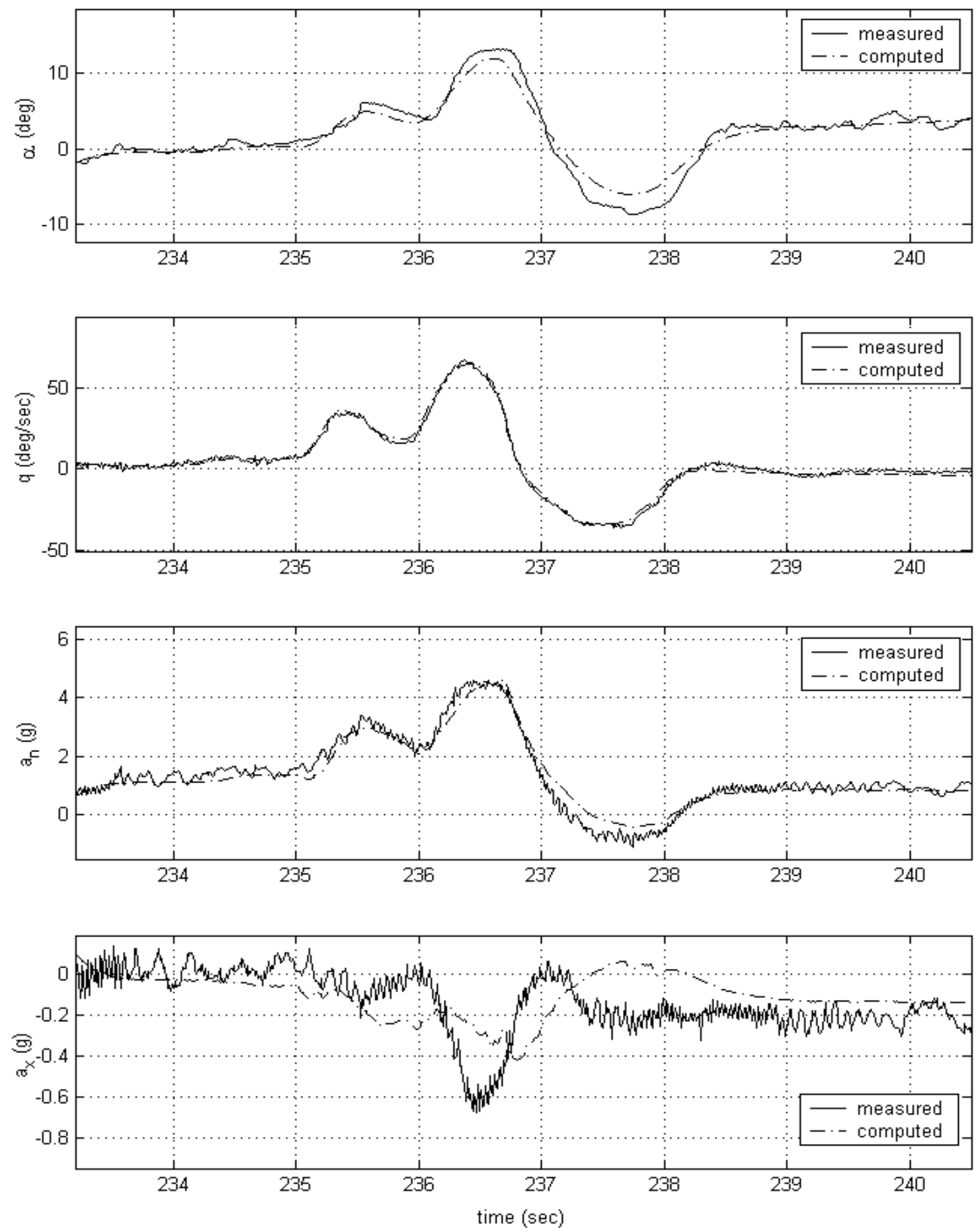

Figure 6.3.6 Comparison of measured and computed time histories from Flight 15, Maneuver 3, longitudinal input ( $\alpha$, pitch rate, normal acceleration, axial acceleration) 
Flight 15, Maneuver 3 (longitudinal input)
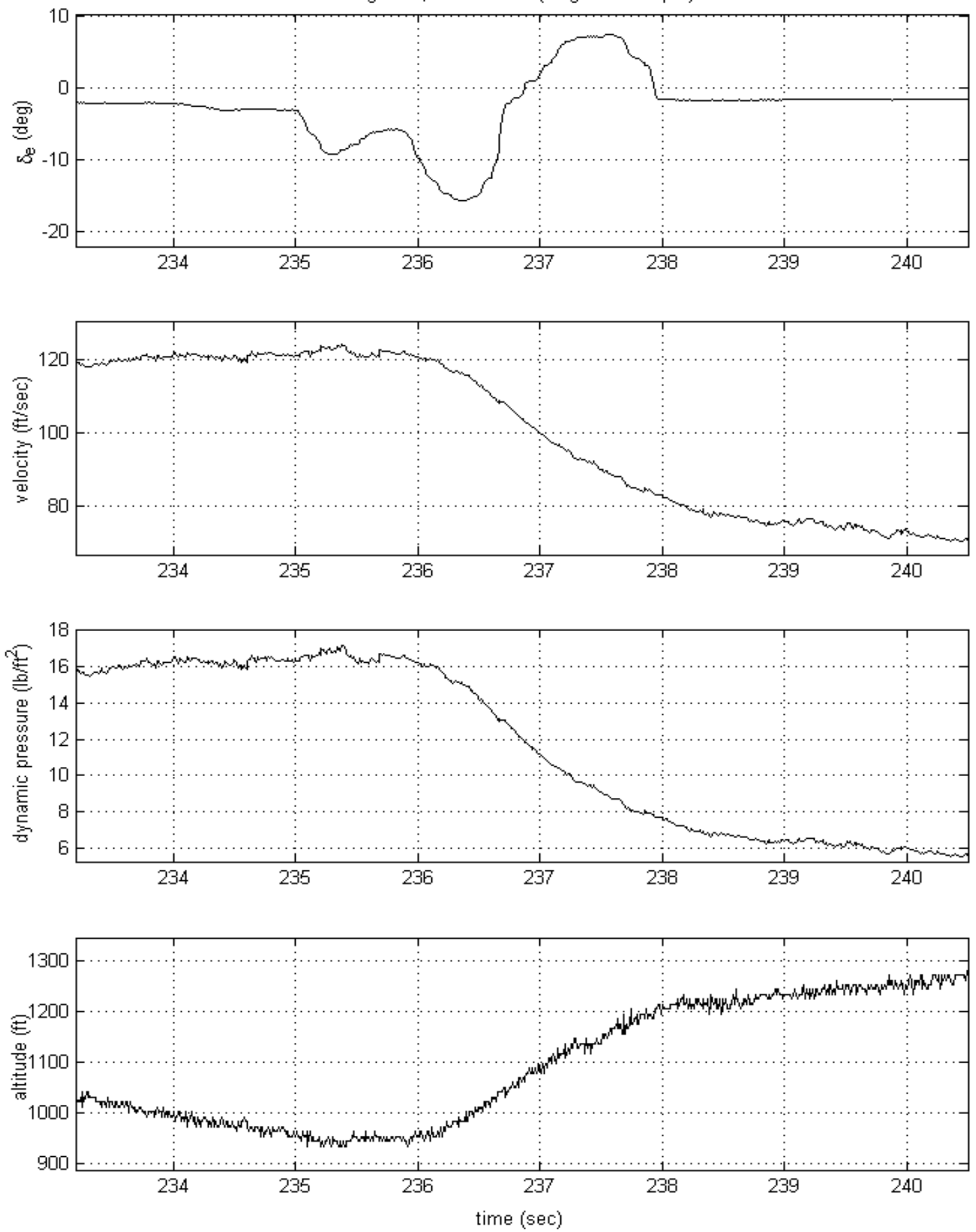

Figure 6.3.7 Comparison of measured and computed time histories from Flight 15, Maneuver 3, longitudinal input ( $\delta_{\mathrm{e}}$, velocity, dynamic pressure, altitude) 
Flight 16, Maneuver 6 (longitudinal input)
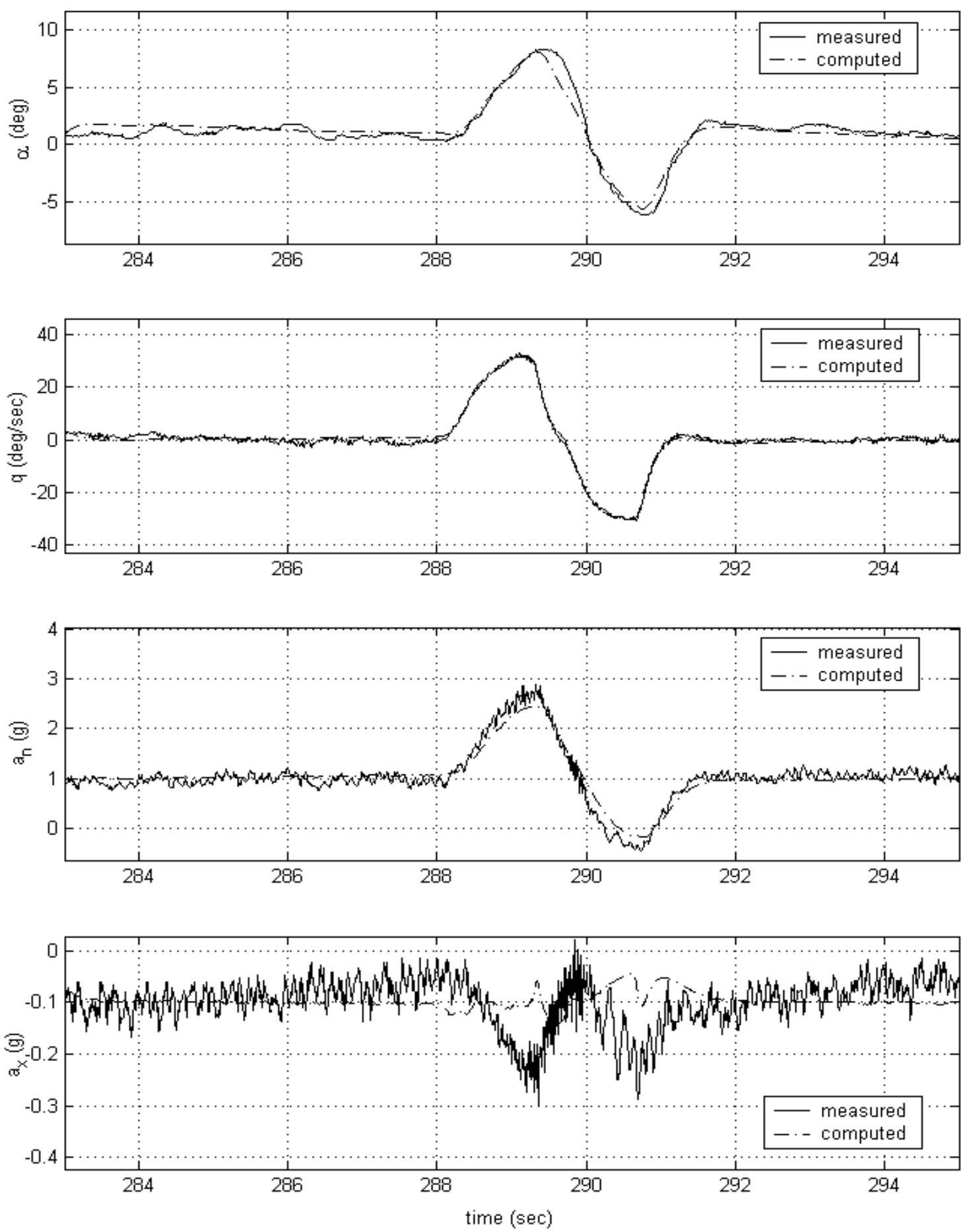

Figure 6.3.8 Comparison of measured and computed time histories from Flight 16, Maneuver 6, longitudinal input ( $\alpha$, pitch rate, normal acceleration, axial acceleration) 
Flight 16, Maneuver 6 (longitudinal input)
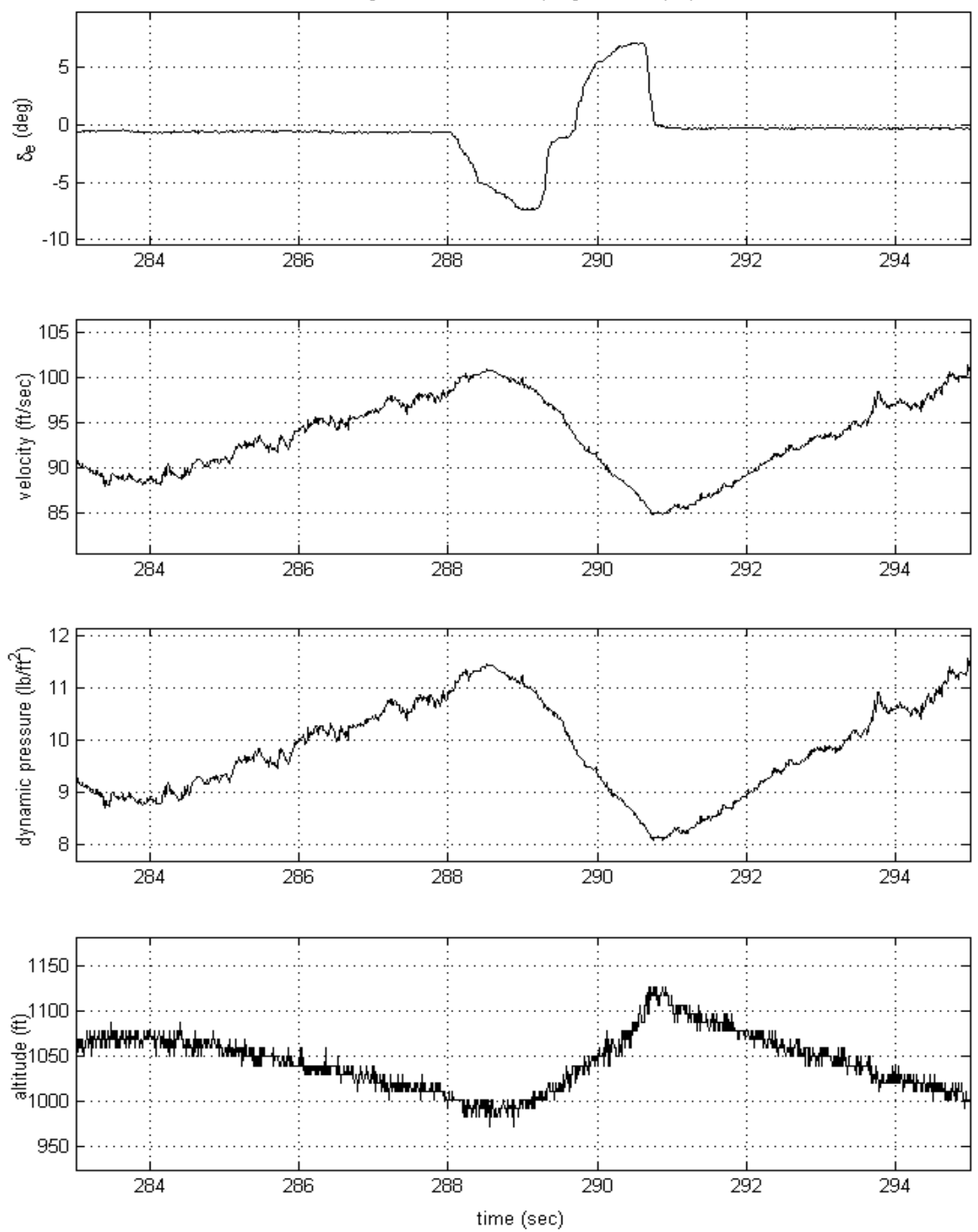

Figure 6.3.9 Comparison of measured and computed time histories from Flight 16, Maneuver 6 , longitudinal input ( $\delta_{\mathrm{e}}$, velocity, dynamic pressure, altitude) 
Flight 17, Maneuver 3 (longitudinal input)
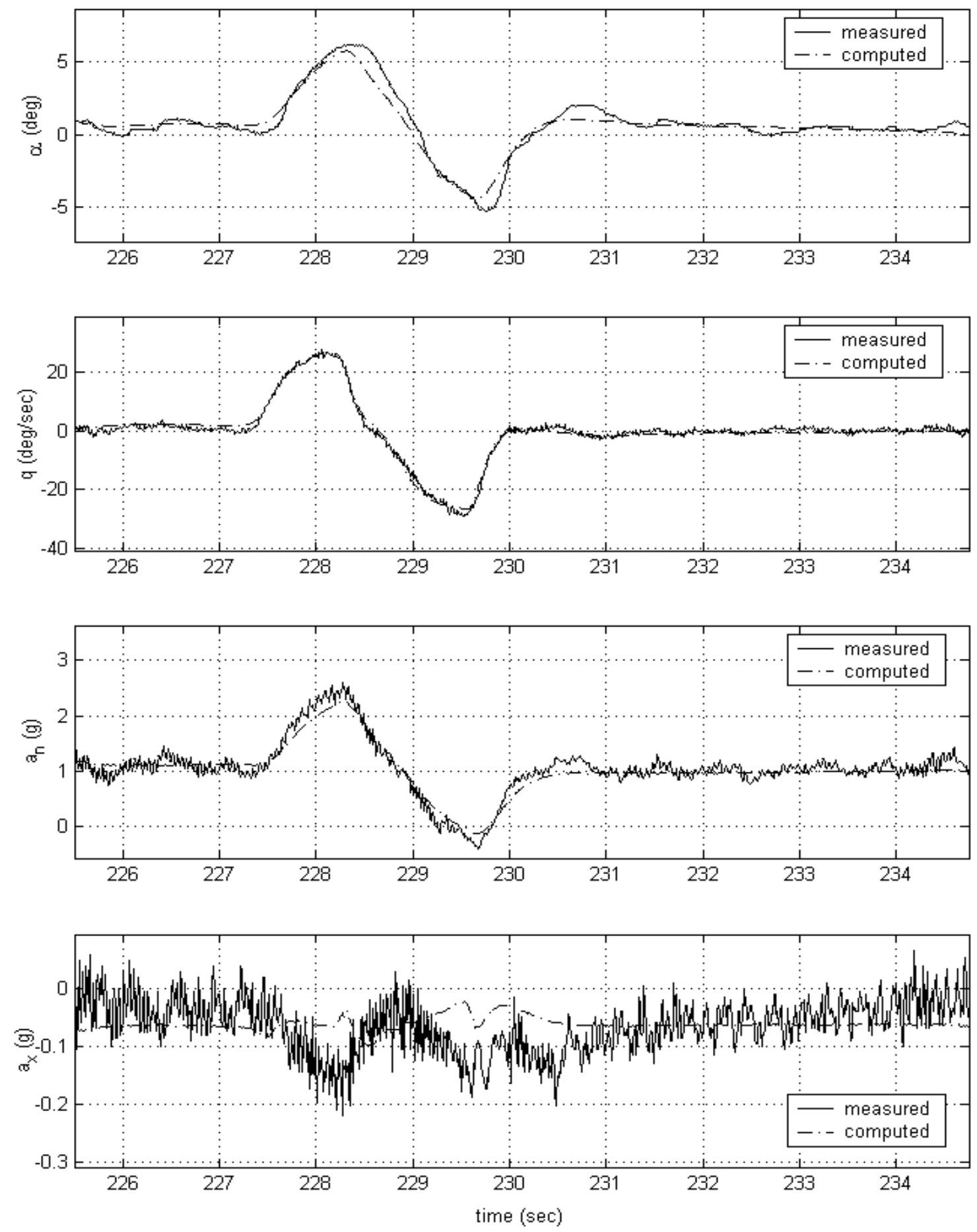

Figure 6.3.10 Comparison of measured and computed time histories from Flight 17, Maneuver 3, longitudinal input ( $\alpha$, pitch rate, normal acceleration, axial acceleration) 
Flight 17, Maneuver 3 (longitudinal input)
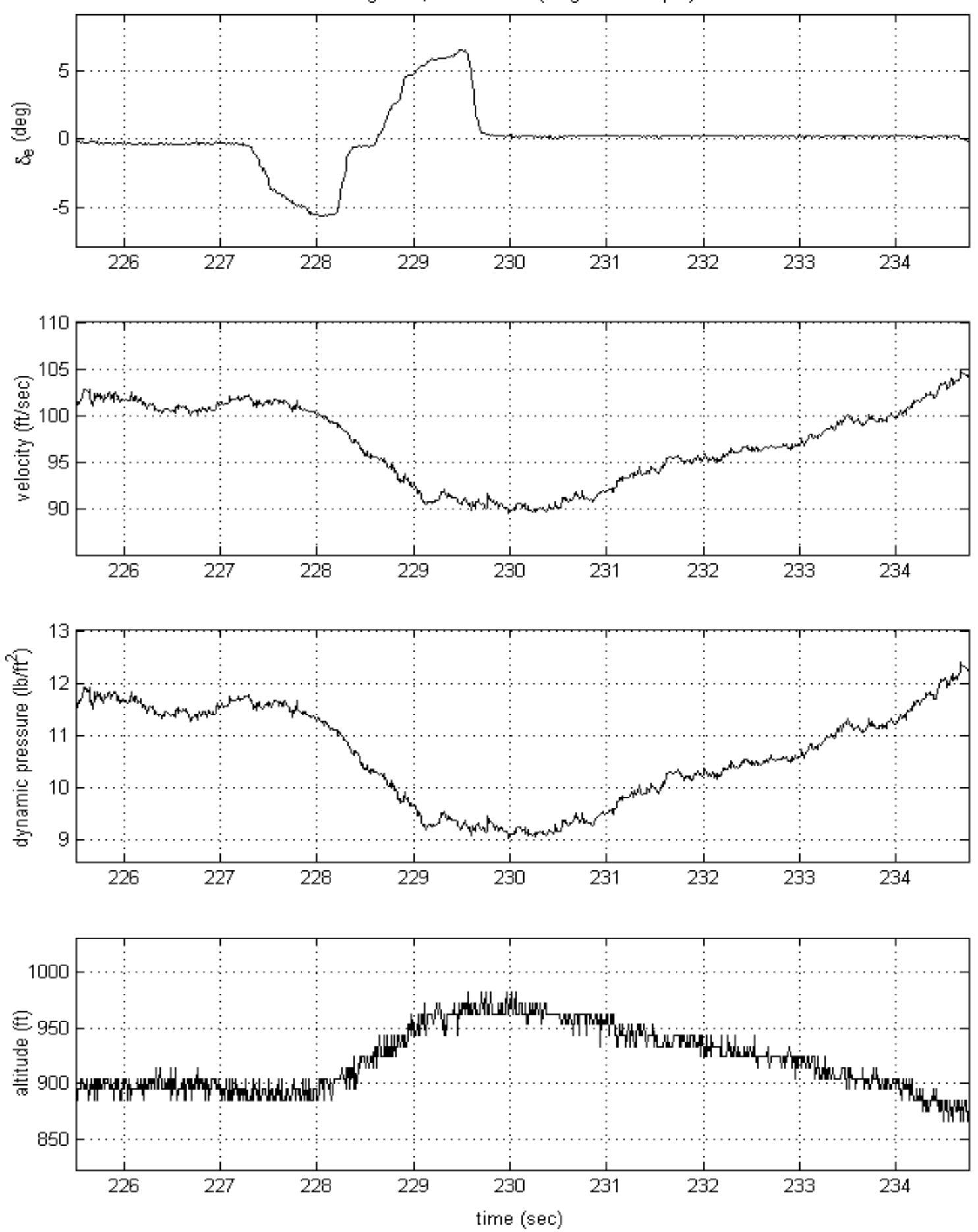

Figure 6.3.11 Comparison of measured and computed time histories from Flight 17, Maneuver 3, longitudinal input ( $\delta_{\mathrm{e}}$, velocity, dynamic pressure, altitude) 
Flight 18, Maneuver 9 (longitudinal input)
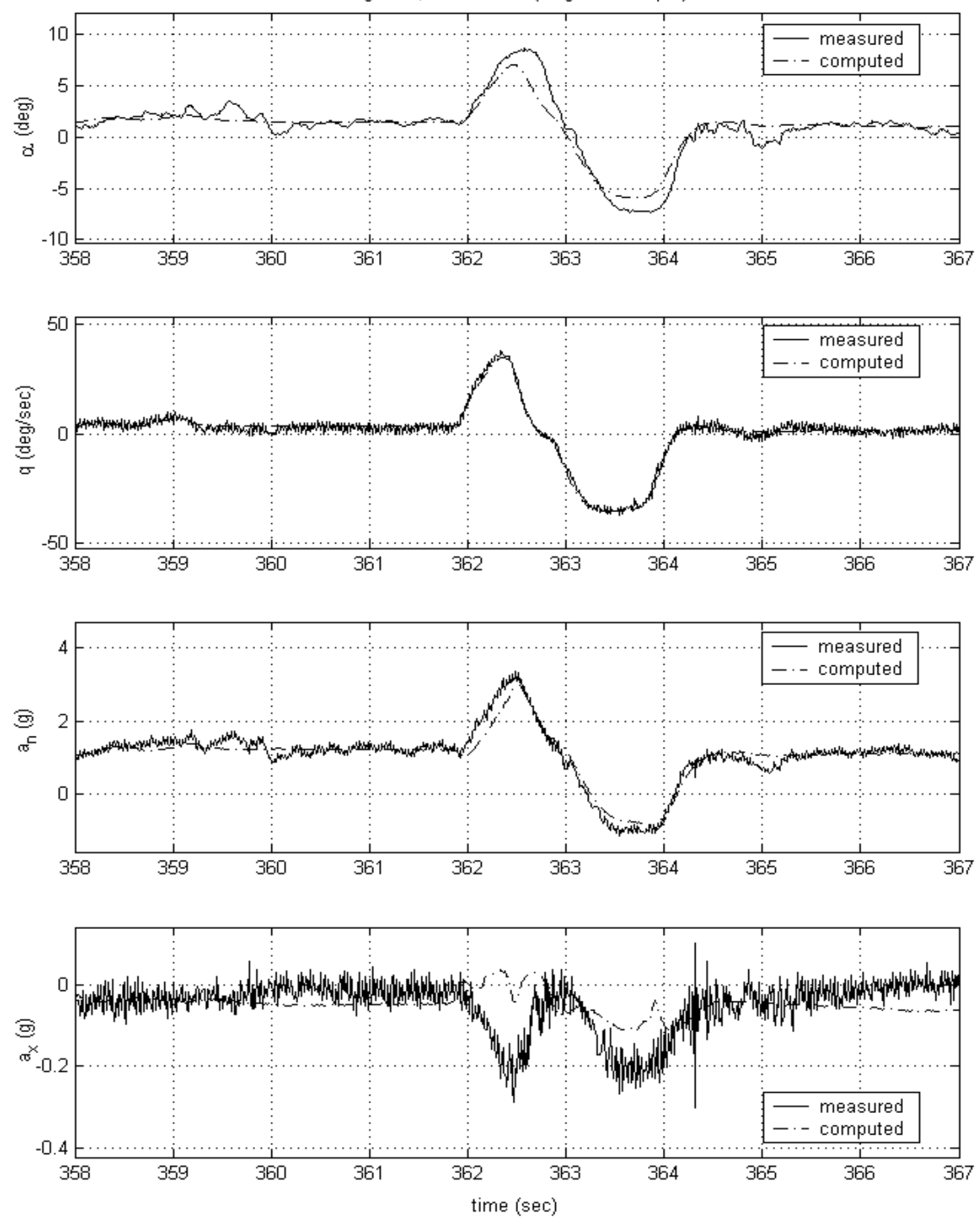

Figure 6.3.12 Comparison of measured and computed time histories from Flight 18, Maneuver 9, longitudinal input ( $\alpha$, pitch rate, normal acceleration, axial acceleration) 
Flight 18, Maneuver 9 (longitudinal input)
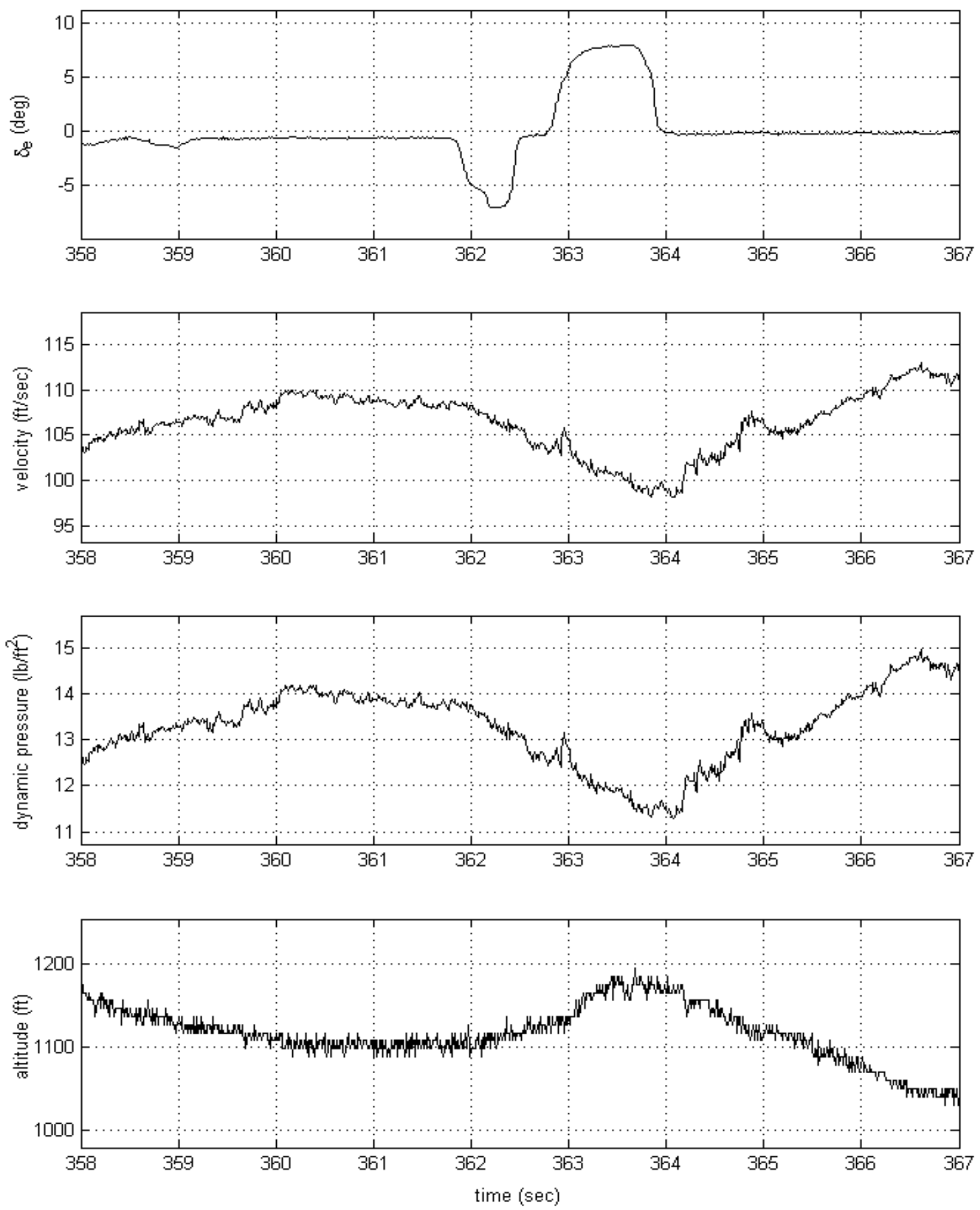

Figure 6.3.13 Comparison of measured and computed time histories from Flight 18, Maneuver 9 , longitudinal input ( $\delta_{\mathrm{e}}$, velocity, dynamic pressure, altitude) 

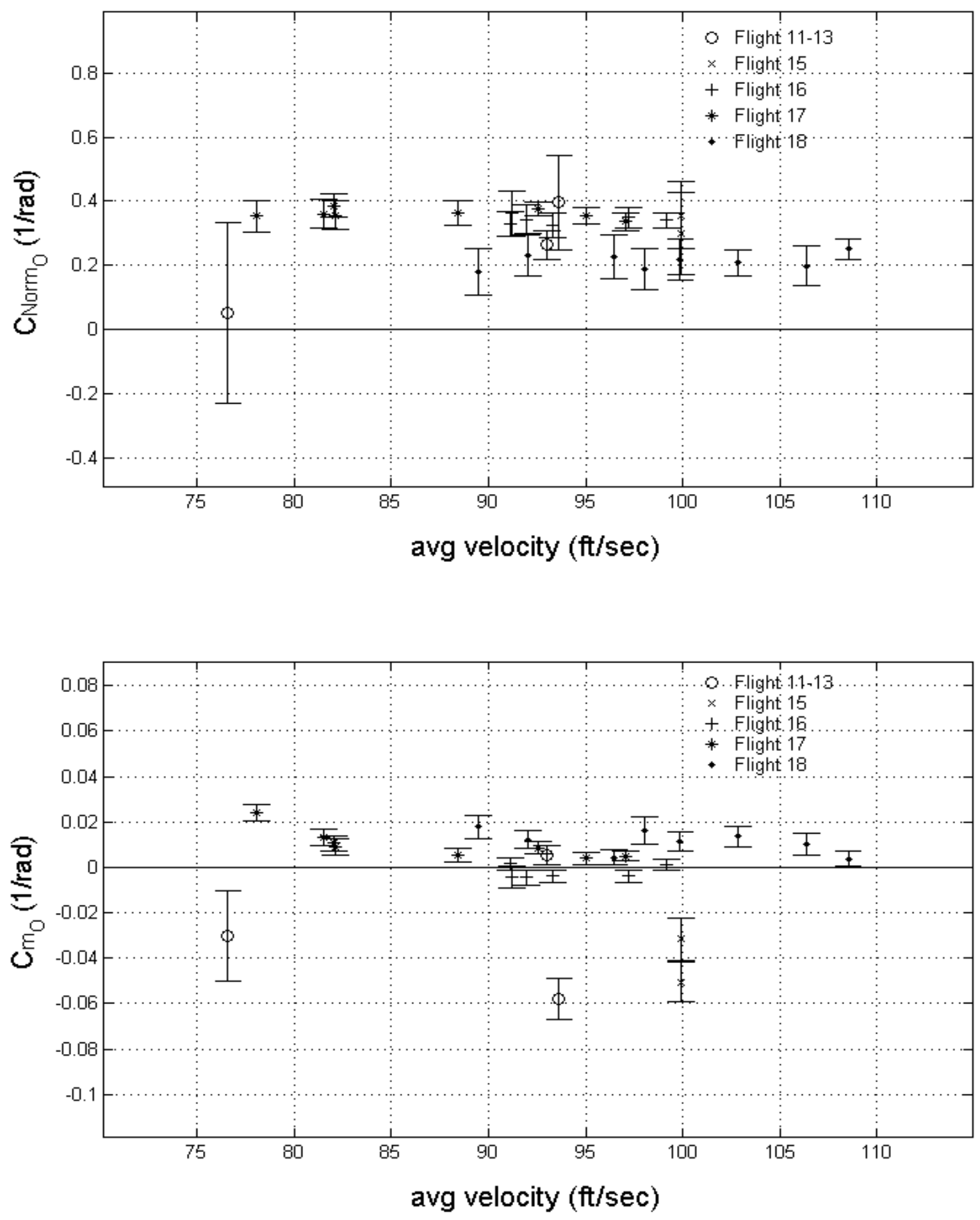

Figure 6.3.14 Off-line estimation results of $\mathrm{C}_{\mathrm{Norm}_{\mathrm{o}}}$ and $\mathrm{C}_{\mathrm{m}_{\mathrm{o}}}$ for longitudinal Flights 11-13, 15, 16, 17, and 18 versus velocity 

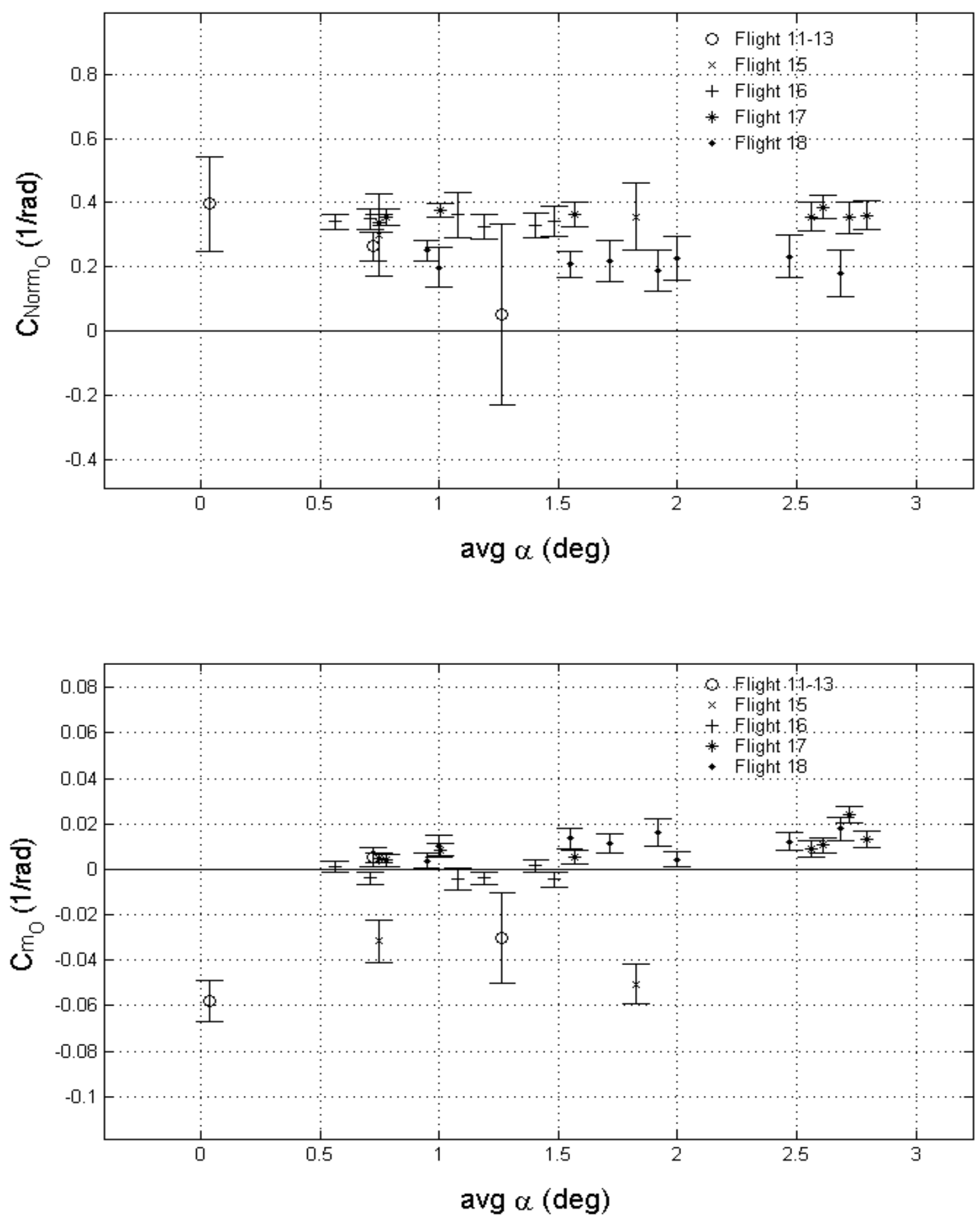

Figure 6.3.15 Off-line estimation results of $\mathrm{C}_{\mathrm{Norm}_{\mathrm{O}}}$ and $\mathrm{C}_{\mathrm{m}_{\mathrm{O}}}$ for longitudinal Flights 11-13, 15, 16, 17, and 18 versus angle of attack 

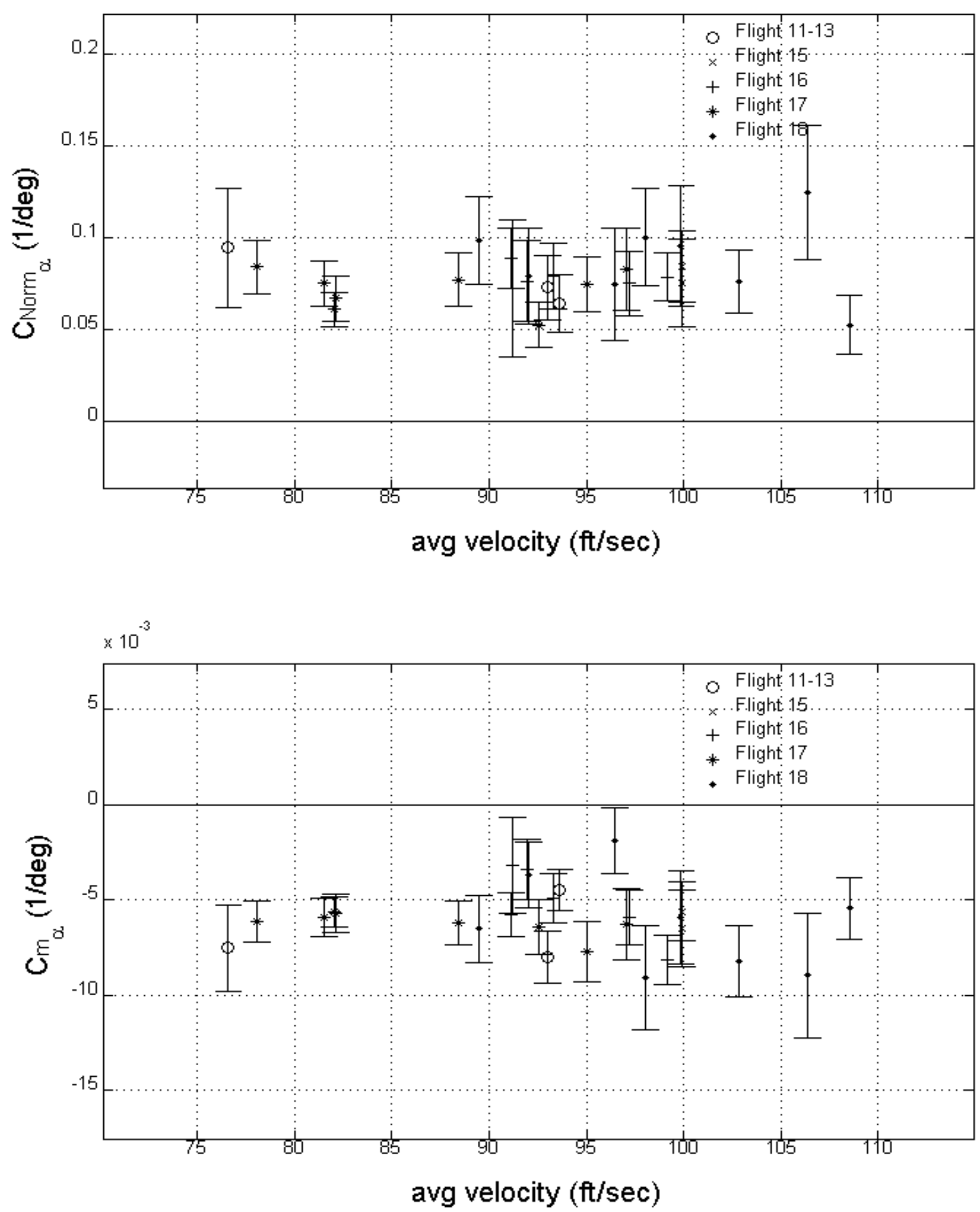

Figure 6.3.16 Off-line estimation results of $\mathrm{C}_{\mathrm{Norm}_{\alpha}}$ and $\mathrm{C}_{\mathrm{m}_{\alpha}}$ for longitudinal Flights 11-13, 15, 16, 17, and 18 versus velocity 

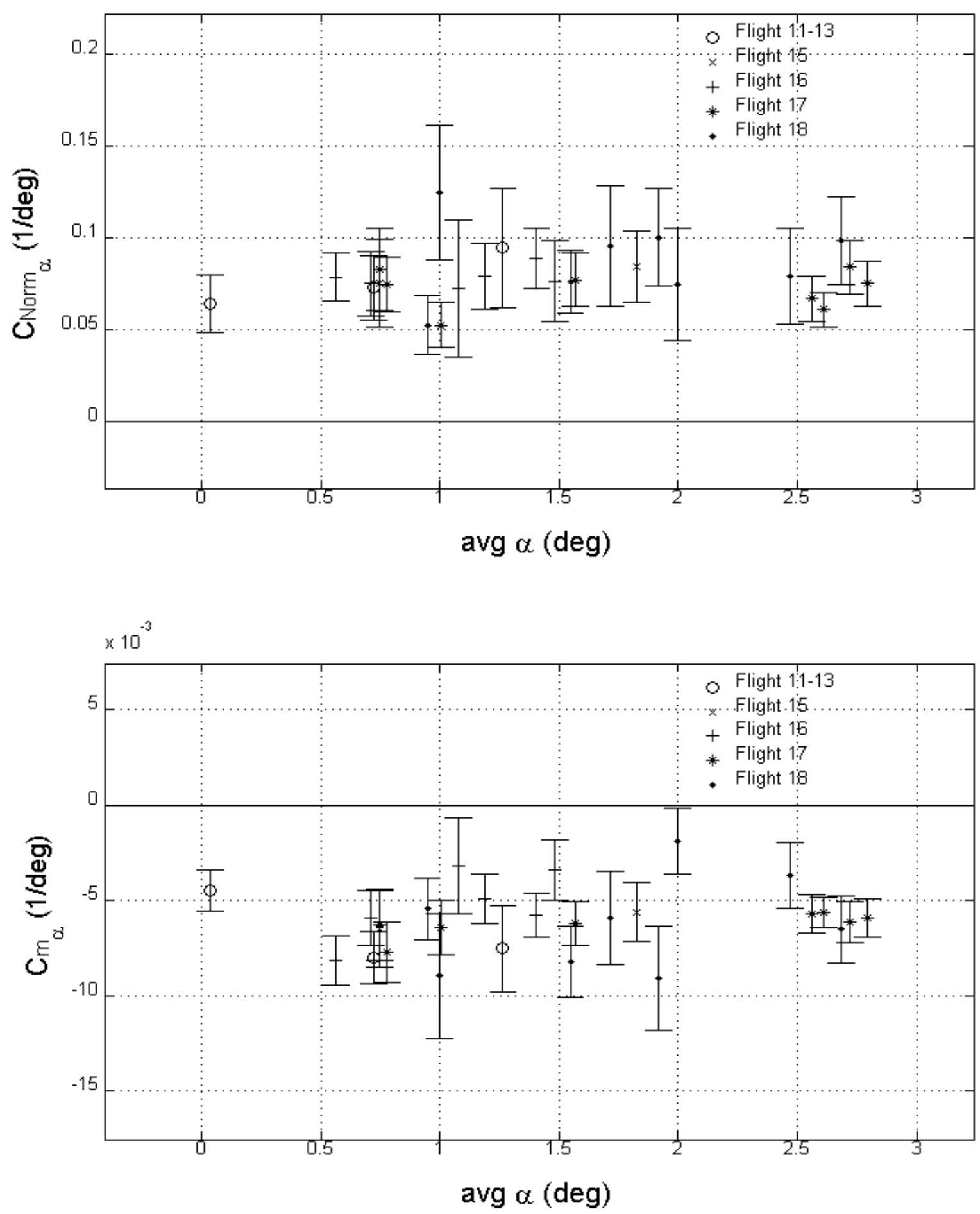

Figure 6.3.17 Off-line estimation results of $\mathrm{C}_{\mathrm{Norm}_{\alpha}}$ and $\mathrm{C}_{\mathrm{m}_{\alpha}}$ for longitudinal Flights 11-13, 15, 16, 17, and 18 versus angle of attack 

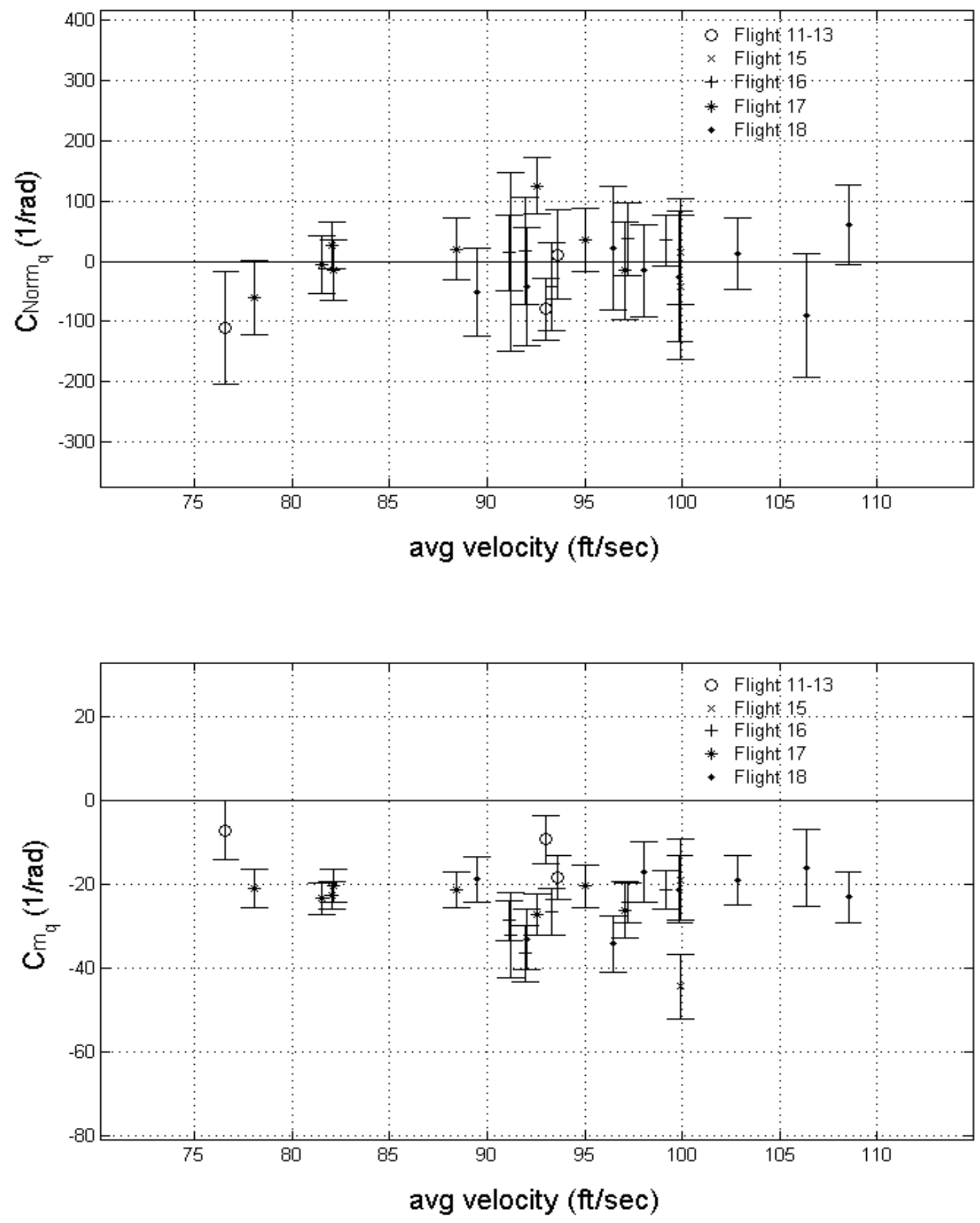

Figure 6.3.18 Off-line estimation results of $\mathrm{C}_{\text {Norm }_{\mathrm{q}}}$ and $\mathrm{C}_{\mathrm{q}}$ for longitudinal Flights $11-13,15,16,17$, and 18 versus velocity 

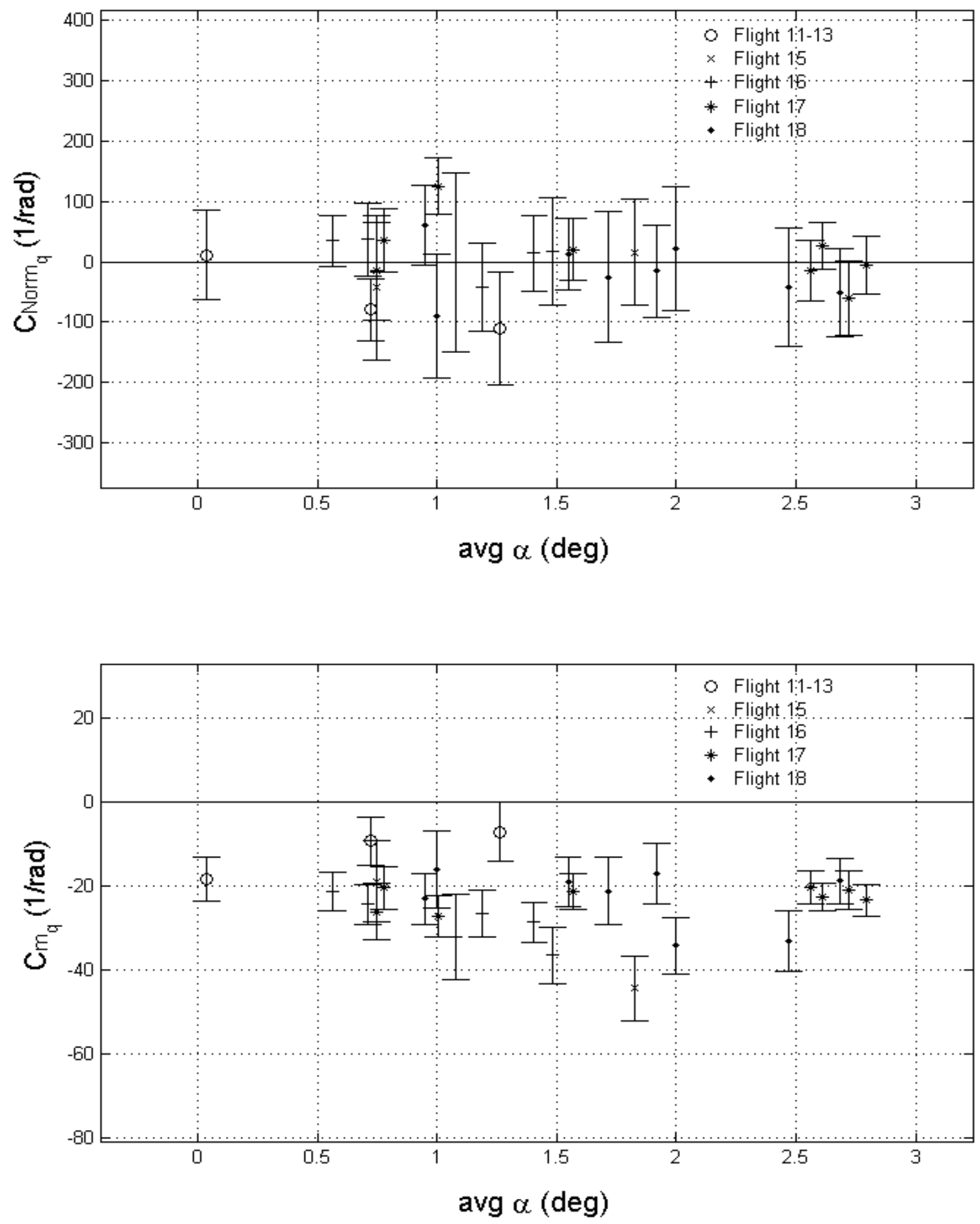

Figure 6.3.19 Off-line estimation results of $\mathrm{C}_{\mathrm{Norm}_{\mathrm{q}}}$ and $\mathrm{C}_{\mathrm{q}}$ for longitudinal Flights 11-13, 15, 16, 17, and 18 versus angle of attack 

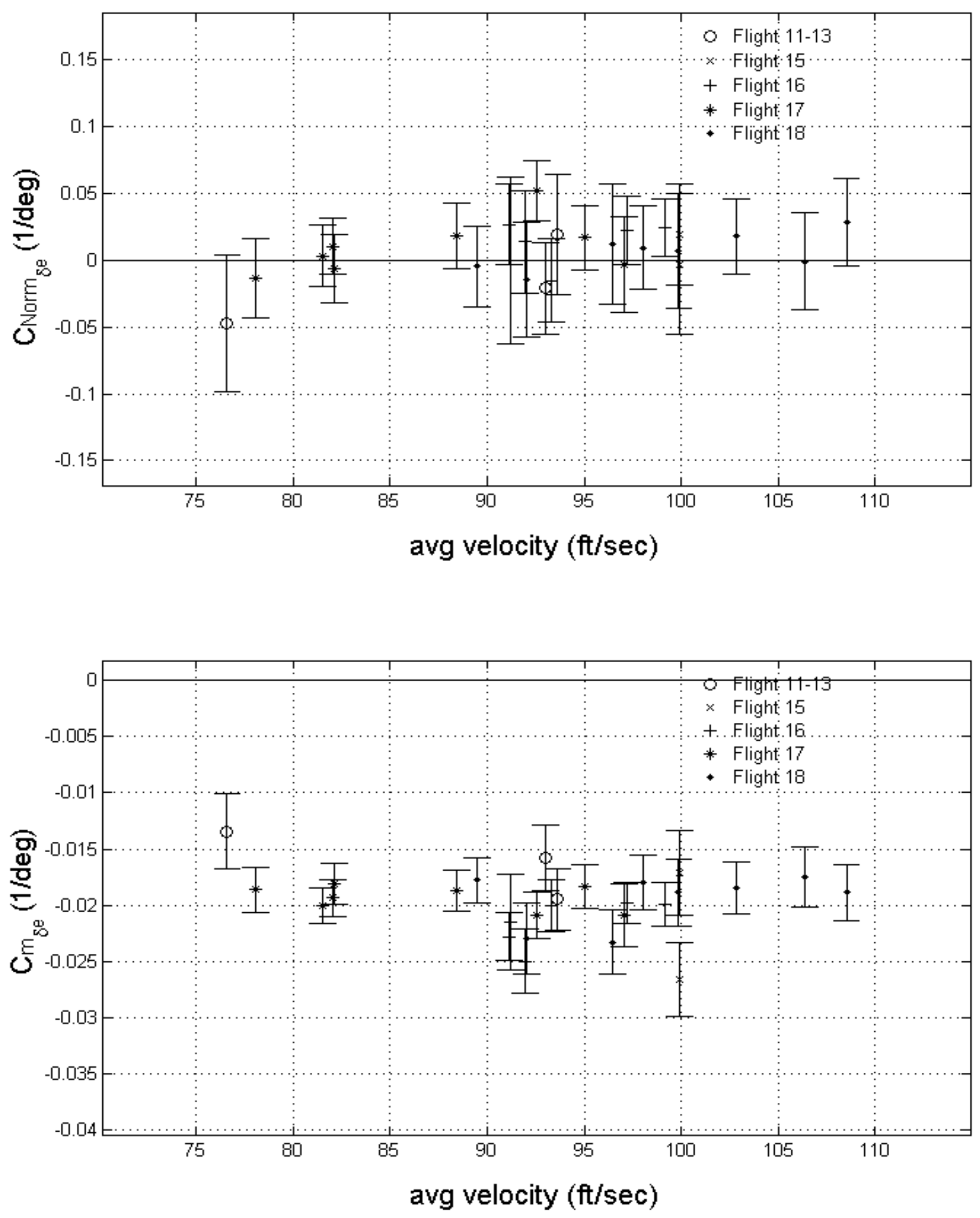

Figure 6.3.20 Off-line estimation results of $\mathrm{C}_{\mathrm{Norm}_{\delta_{\mathrm{e}}}}$ and $\mathrm{C}_{\mathrm{m}_{\delta_{\mathrm{e}}}}$ for longitudinal Flights $11-13,15,16,17$, and 18 versus velocity 

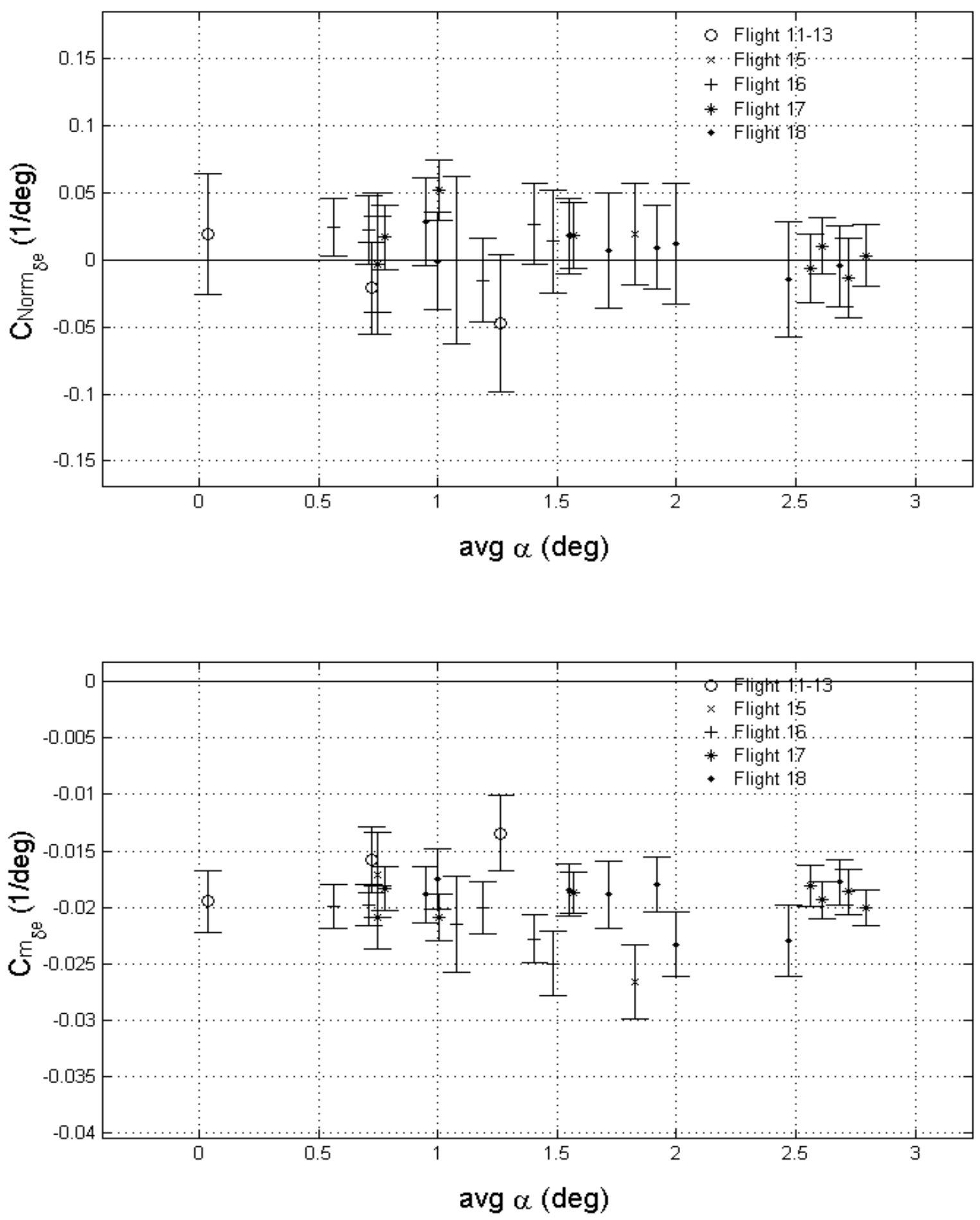

Figure 6.3.21 Off-line estimation results of $\mathrm{C}_{\mathrm{Norm}_{\delta_{e}}}$ and $\mathrm{C}_{\mathrm{m}_{\delta_{\mathrm{e}}}}$ for longitudinal Flights 11-13, 15, 16, 17, and 18 versus angle of attack 

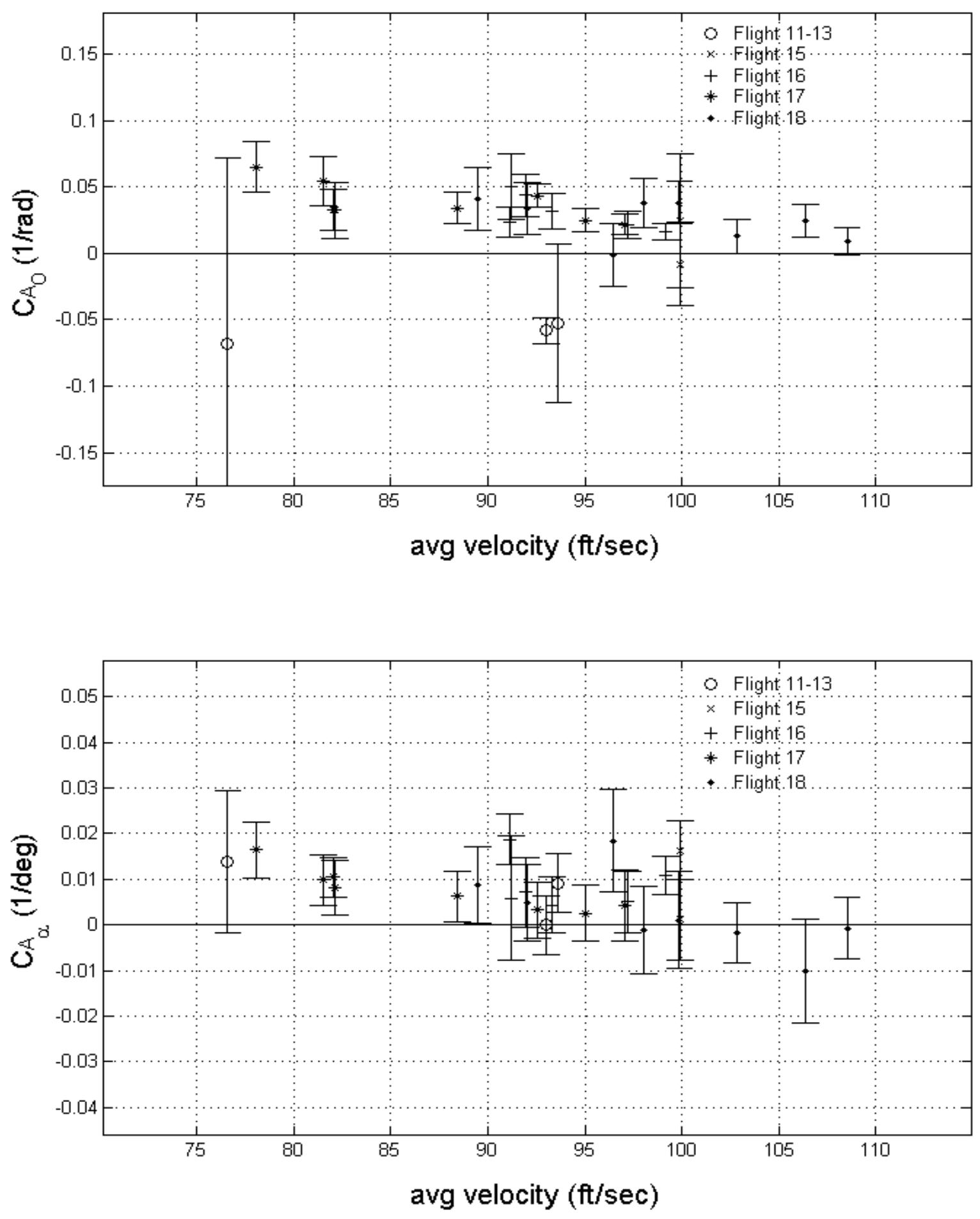

Figure 6.3.22 Off-line estimation results of $\mathrm{C}_{\mathrm{A}_{\mathrm{O}}}$ and $\mathrm{C}_{\mathrm{A}_{\alpha}}$ for longitudinal Flights 11-13, 15, 16, 17, and 18 versus velocity 

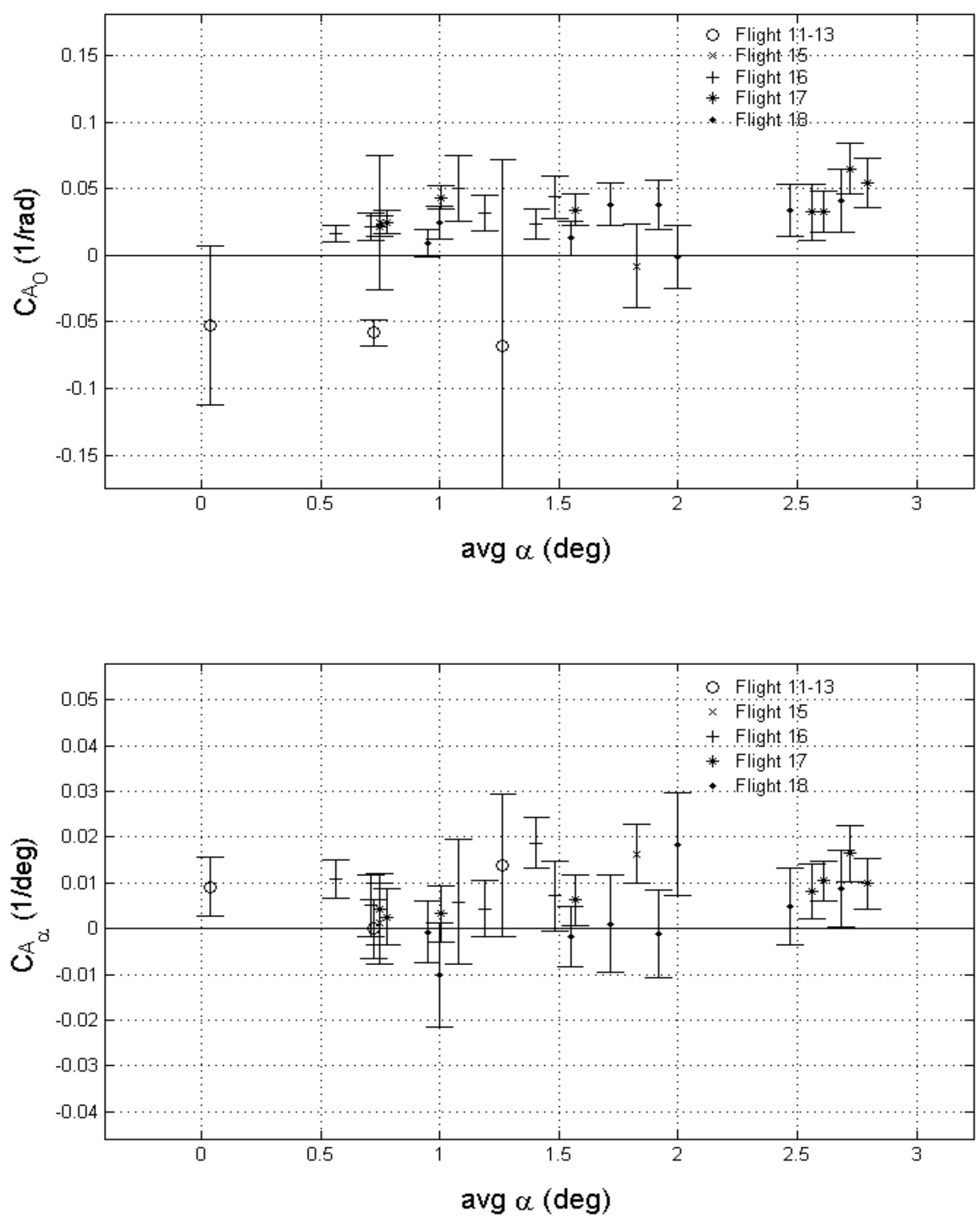

Figure 6.3.23 Off-line estimation results of $\mathrm{C}_{\mathrm{A}_{\mathrm{O}}}$ and $\mathrm{C}_{\mathrm{A}_{\alpha}}$ for longitudinal Flights 11-13, 15, 16, 17, and 18 versus angle of attack 

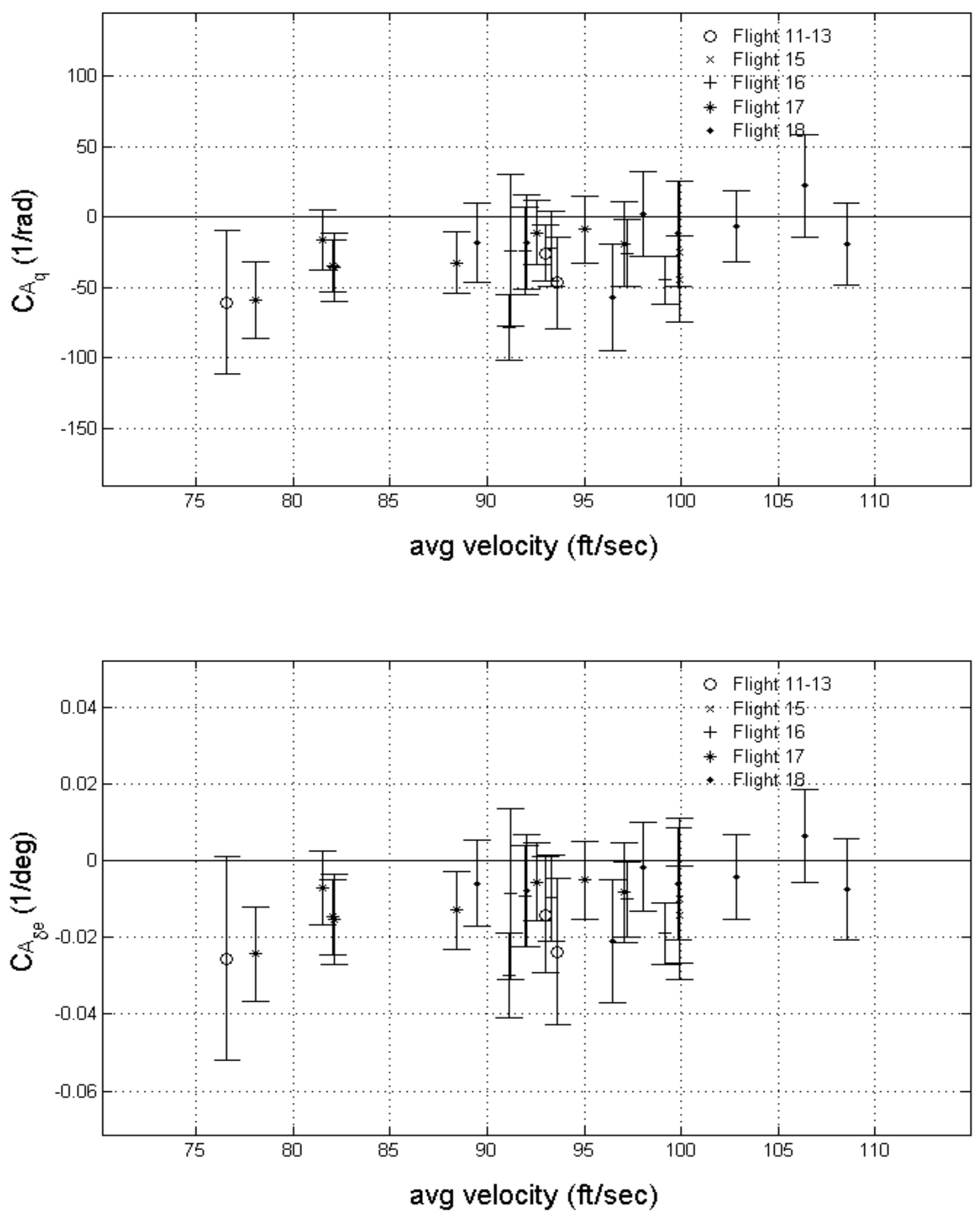

Figure 6.3.24 Off-line estimation results of $\mathrm{C}_{\mathrm{A}_{\mathrm{q}}}$ and $\mathrm{C}_{\mathrm{A}_{\mathrm{e}_{\mathrm{e}}}}$ for longitudinal Flights $11-13,15,16,17$, and 18 versus velocity 

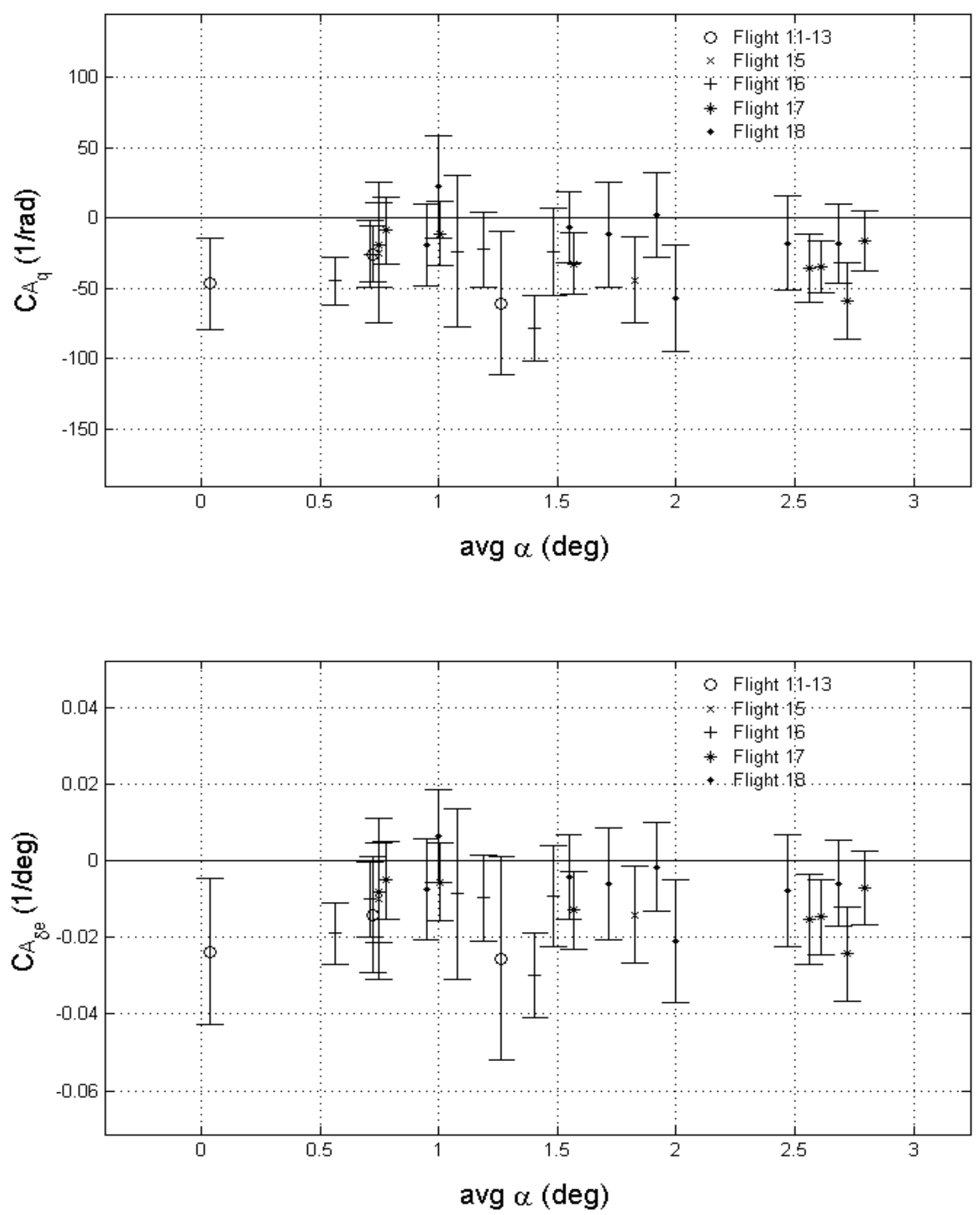

Figure 6.3.25 Off-line estimation results of $\mathrm{C}_{\mathrm{A}_{\mathrm{q}}}$ and $\mathrm{C}_{\mathrm{A}_{\mathrm{e}_{\mathrm{e}}}}$ for longitudinal Flights 11-13, 15, 16, 17, and 18 versus angle of attack 

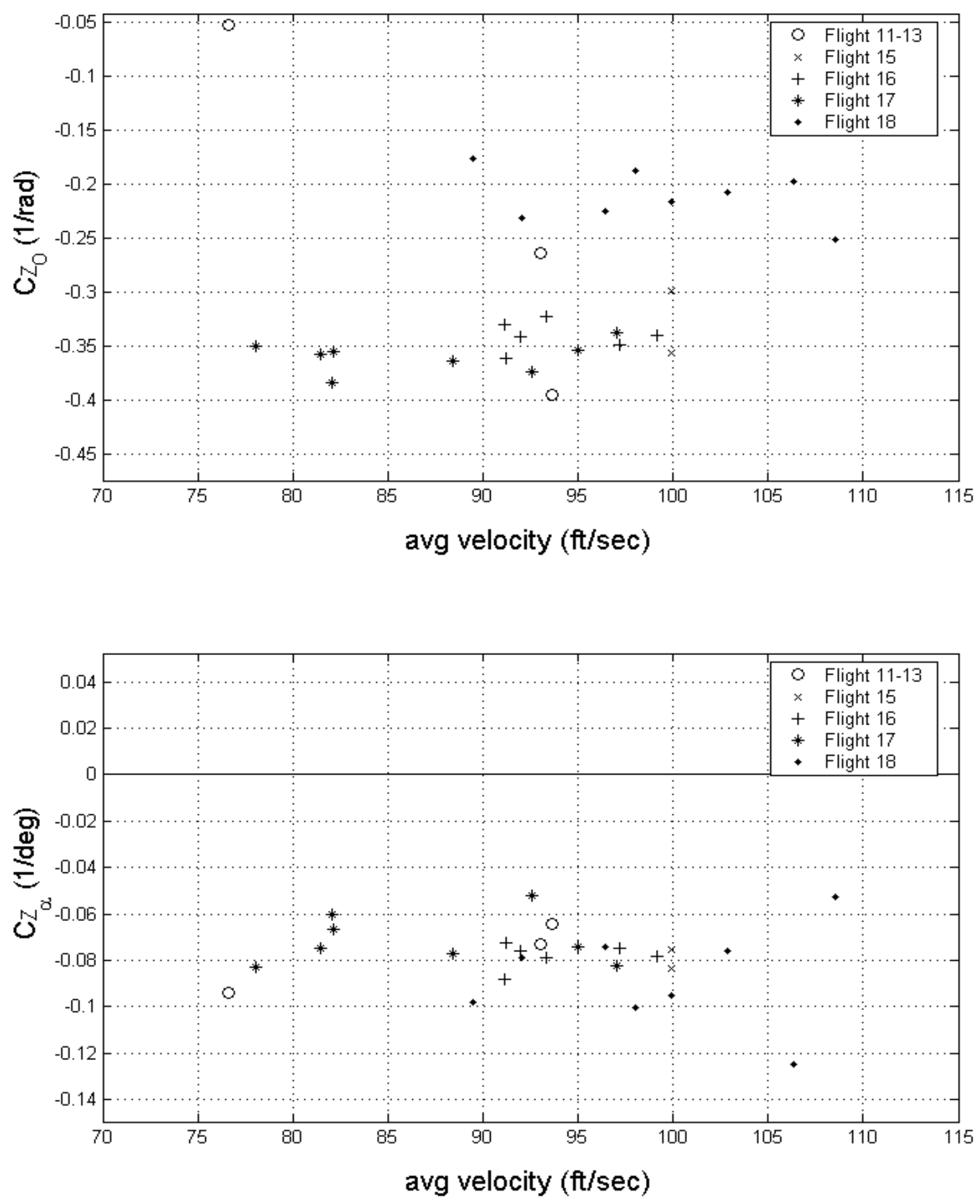

Figure 6.3.26 Off-line estimation results of $\mathrm{C}_{\mathrm{Z}_{\mathrm{O}}}$ and $\mathrm{C}_{\mathrm{Z}_{\alpha}}$ for longitudinal Flights $11-13,15,16,17$, and 18 versus velocity 

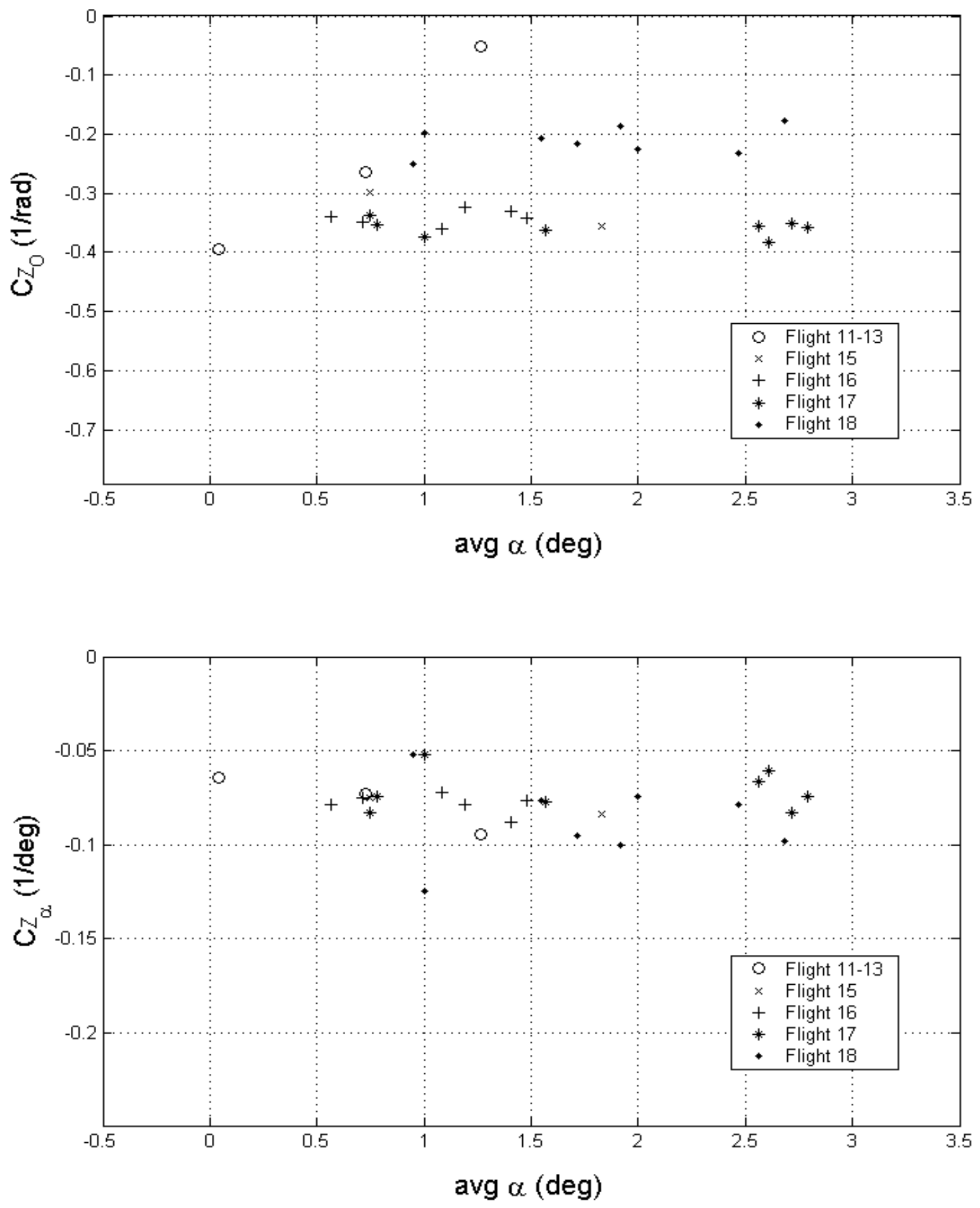

Figure 6.3.27 Off-line estimation results of $\mathrm{C}_{\mathrm{Z}_{\mathrm{O}}}$ and $\mathrm{C}_{\mathrm{Z}_{\alpha}}$ for longitudinal Flights 11-13, 15, 16, 17, and 18 versus angle of attack 

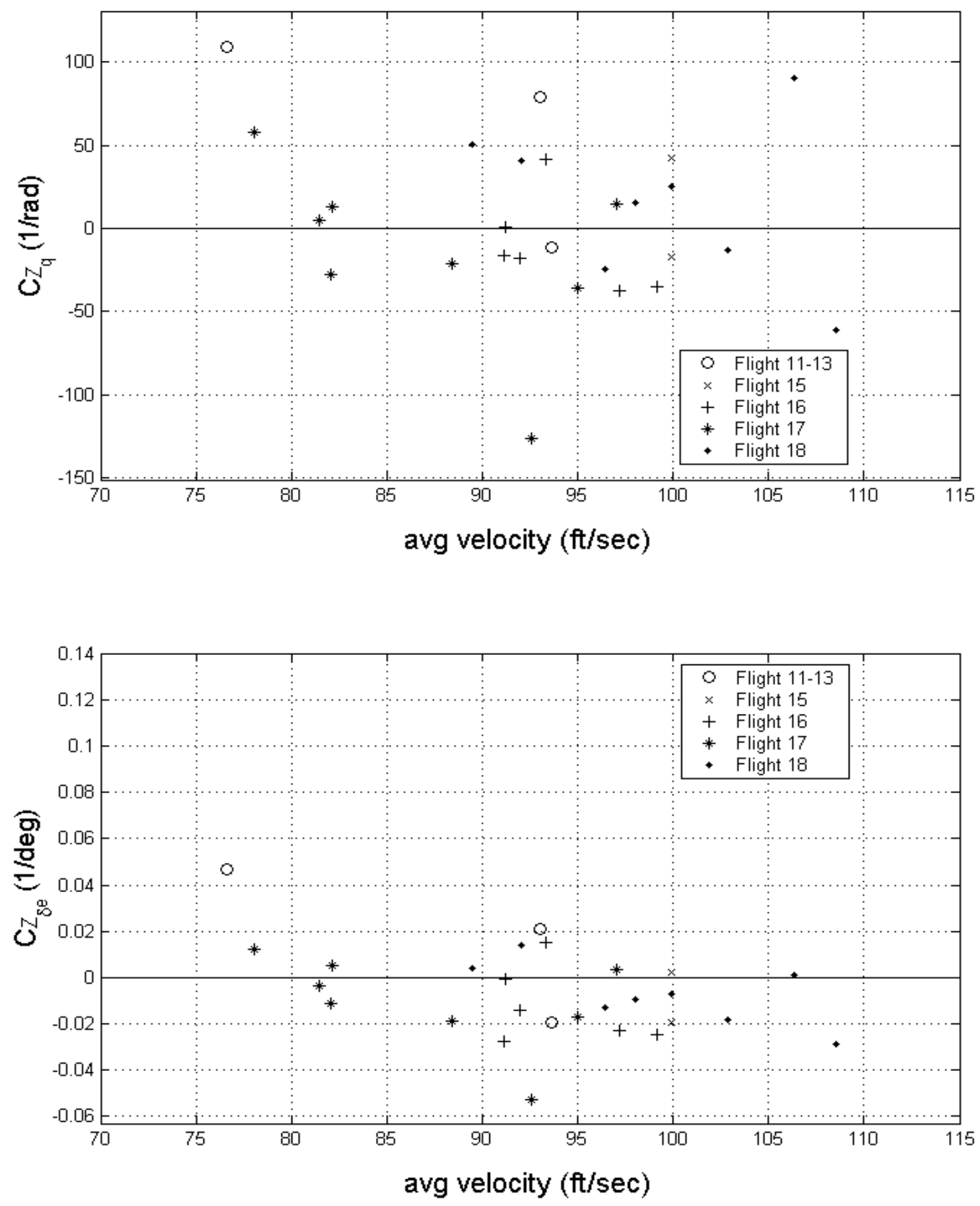

Figure 6.3.28 Off-line estimation results of $\mathrm{C}_{\mathrm{Z}_{\mathrm{q}}}$ and $\mathrm{C}_{\mathrm{Z}_{\delta_{\mathrm{e}}}}$ for longitudinal Flights $11-13,15,16,17$, and 18 versus velocity 

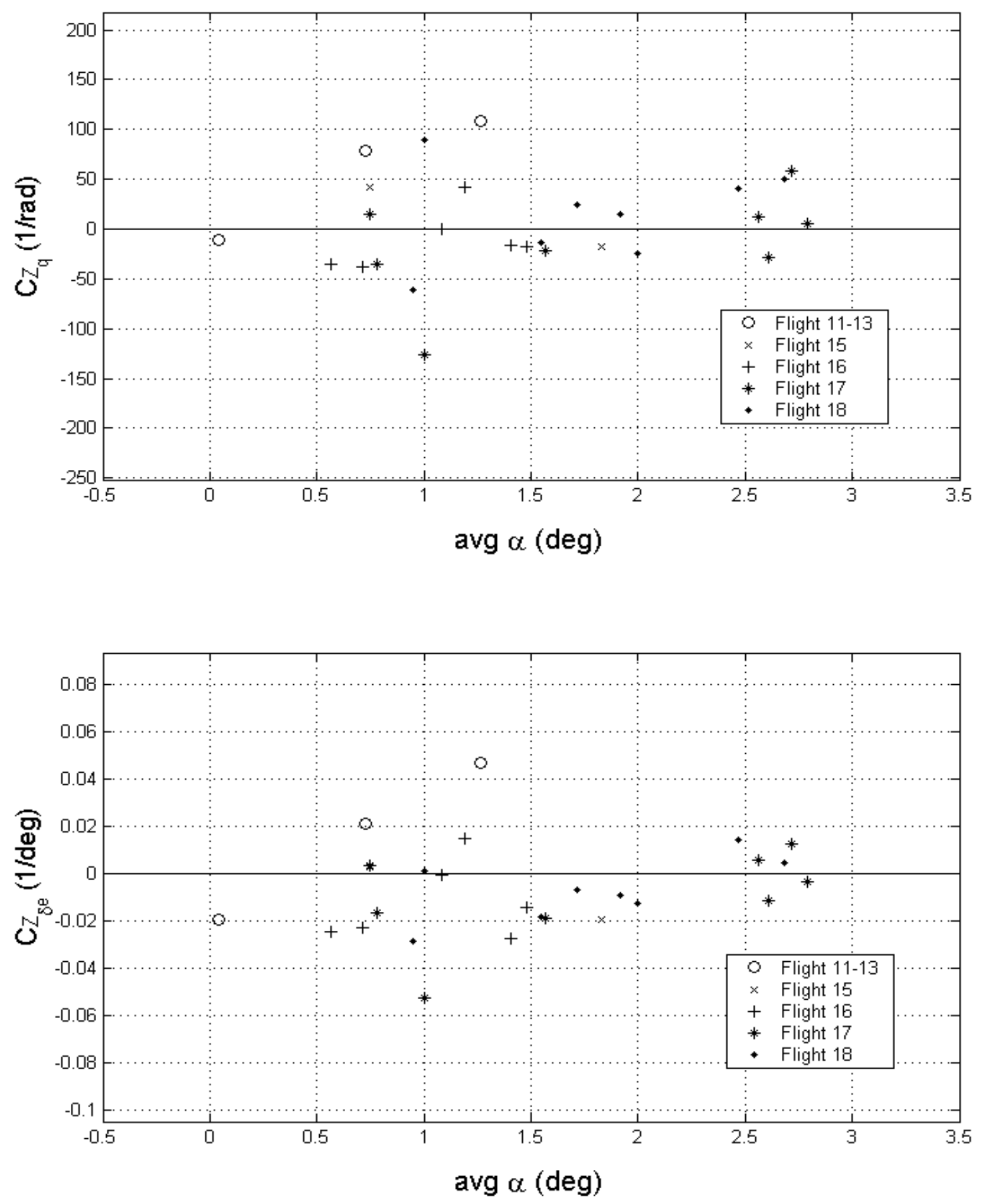

Figure 6.3.29 Off-line estimation results of $\mathrm{C}_{\mathrm{Z}_{\mathrm{q}}}$ and $\mathrm{C}_{\mathrm{Z}_{\delta_{\mathrm{e}}}}$ for longitudinal Flights $11-13,15,16,17$, and 18 versus angle of attack 


\subsection{Lateral-Directional Results}

Figures 6.4.1-6.4.59 represent the results for the lateral-directional stability and control derivatives of the B777 model. They are presented in terms of estimates of aerodynamic coefficients and the relative time history comparisons between the measured and computed aircraft responses. Estimates were obtained for the rolling moment, yawing moment, and lateral force $\left(\mathrm{C}_{1}, \mathrm{C}_{\mathrm{n}}\right.$, and $\left.\mathrm{C}_{\mathrm{y}}\right)$. Each estimate was presented with a corresponding Cramer-Rao bound represented as a vertical bar for each flight maneuver test point. The data points are categorized by flight test; therefore it can be seen which flights produced that particular lateral-directional maneuver. All figures discussed are located at the end of this section.

Figures 6.4.1-6.4.3 shows a sample time history collected for lateral-directional Flight $\mathrm{C}$ aircraft parameters from takeoff to landing. Data parameters include; $\beta$, roll rate, yaw rate, lateral acceleration, $\alpha$, velocity, dynamic pressure, and altitude, along with the corresponding control surface deflections $\left(\delta_{\mathrm{e}}, \delta_{\mathrm{a}}, \delta_{\mathrm{r}}\right)$. Typical average maneuver ranges for the roll rate were $\pm 60 \mathrm{deg} / \mathrm{sec}$, yaw rates of $\pm 40 \mathrm{deg} / \mathrm{sec}$, and lateral accelerations were found within $\pm 0.5 \mathrm{~g}$ 's. In Figures 6.4.1-6.4.3 the first 80 plus seconds show the model aircraft sitting on the runway with the engines running. For each engine start, some time was taken to make sure they were tuned properly before each launch. The descent-landing phase can be seen around at 470 seconds into the flight history file. All angular rates were displayed in degrees/second, accelerations in g's, airspeed in feet/second, altitude in feet, along with measured flow angles in degrees. Figure 6.4.3 shows the time history of each of the three major control surfaces for Flight C. Instances of data signal loss were not a common occurrence, but for Flight $\mathrm{C}$ in particular, the rudder channel (see Figure 6.4.3) specifically shows data loss between maneuvers 8 and 11 and was regained for the last maneuver performed.

Figures 6.4.4-6.4.17 show maneuver comparisons of measured and computed time histories collected from each of the lateral-directional flights, specifically:

- Flight A, Maneuver 8

- Flight B, Maneuver 8

- Flight C, Maneuver 7

- Flight D, Maneuver 6
(Figures 6.4.4-6.4.6)

(Figures 6.4.7-6.4.8)

(Figures 6.4.9-6.4.11)

(Figures 6.4.12-6.4.14) 
- Flight E, Maneuver 10

(Figures 6.4.15-6.4.17)

The outputs from the estimation process show the $\beta$, roll rate, yaw rate, and lateral acceleration. The associated control inputs $\delta_{\mathrm{a}}$ and $\delta_{\mathrm{r}}$; along with $\alpha$, velocity, dynamic pressure, and altitude are shown in secondary sets of figures. Maneuver responses shown for the lateral-directional inputs were again selected randomly, one maneuver from each of the five flights completed. Overall, the computed responses for sideslip angle; roll rate, yaw rate, and lateral acceleration gave reasonable matches for each of the maneuvers. During Flights $\mathrm{A}, \mathrm{B}$, and $\mathrm{C}$, combined $\delta_{\mathrm{a}} / \delta_{\mathrm{r}}$ maneuvers were typically executed while Flights D and E featured a mixture of individual aileron and rudder pulses, as well as combination maneuvers.

An example maneuver from Flight B, Figure 6.4.7 shows the measured and computed responses, along with the corresponding control inputs in Figure 6.4.8. Nose cone sensor information, including angle of attack, sideslip and airspeed were lost during the Flight $\mathrm{B}$ testing due to a connection malfunction. Aircraft data was limited to accelerations, aircraft rates, and control surface information only. For this analysis purposes, Flight B information was not discarded; but average values for the missing flight parameters were used during the iterative estimation process. The roll and yaw rates, along with lateral acceleration were used as output responses during the estimation process. It was the intension to gain a mean value for the overall set of Flight B maneuvers. Then overlay this single date value when compared with the rest of lateraldirectional maneuvers. Typically indispensable signals for PID analysis are control surface deflections, angular rates and linear accelerations which made it possible to use this existing flight information. Flow angles and air data time histories information provides a more accurate modeling of the lateral aircraft dynamics.

Figures 6.4.18-6.4.25 show the lateral estimates obtained plotted against a corresponding maneuver number for Flight B. Overall the rolling moment and sideforce coefficients provided the most consistent results between Flight B maneuvers. Within this particular flight, all maneuvers were comprised of the combination aileron and rudder inputs. A mean value estimate, indicated by the dotted line, for each set of figures was plotted. This value was then transferred onto the final set of estimate figures and compared with all other lateral maneuvers collected. 
Figures 6.4.26-6.4.59 show the off-line estimation results plotted versus the average velocity and average sideslip angle for all the lateral-directional estimates. Overall, two sets of plots were generated (4 total for each estimate); set one includes the estimates plotted against the average velocity and sideslip for all lateral maneuvers. A second set was then created which excluded certain $\delta_{r}$ maneuvers performed in Flight E. Since the response matches for these particular rudder maneuvers faired very well, it was felt that they should be initially included within the results. However, these particular estimates usually overshadowed the remaining estimates, making it difficult visually for evaluating the final results. As an example, Figure 6.4.30 shows values of $\mathrm{C}_{\mathrm{n} \beta}$, and in this case clearly three of the maneuvers overshadow the other estimates. A note was placed within the figure titles when these three estimates were excluded for visual comparison.

Figures 6.4.26-6.4.29 show the lateral aerodynamic biases $\mathrm{C}_{\mathrm{l}_{\mathrm{O}}}$ and $\mathrm{C}_{\mathrm{n}_{\mathrm{O}}}$ estimates hovering above and below the zero axis, these non-zero values could be possibly attributed to the left and right geometric dihedral angle $\left(\Gamma_{1} \neq \Gamma_{\mathrm{r}}\right)$ not being exactly the same. Figures 6.4.30-6.4.33 represent the rolling and yawing moment effects due to sideslip $\left(\mathrm{C}_{\mathrm{l}_{\beta}}\right.$ and $\left.\mathrm{C}_{\mathrm{n} \beta}\right)$. However, the tight grouping of estimates were found between the 105 to $115 \mathrm{ft} / \mathrm{sec}$ range showing that the dihedral effect did not seem to indicate the same variations as found with the aerodynamic bias estimates. Values for sideslip did show that the vehicle was found to be directionally stable throughout the airspeed range, in Figures 6.4.32-6.4.33. Figures 6.4.34-6.4.37 represent the rolling damping and yawing moment estimates due to the roll rate $\left(\mathrm{C}_{\mathrm{l}_{\mathrm{p}}}\right.$ and $\left.\mathrm{C}_{\mathrm{n}_{\mathrm{p}}}\right)$. Overall the roll-damping coefficient remained constant over the airspeed and sideslip ranges in Figures 6.4.366.4.37. However, the Flight B mean value obtained did not fall within the other flight maneuvers. Figures 6.4.38-6.4.41 represent the rolling and yawing damping estimates due to the yaw rate $\left(\mathrm{C}_{\mathrm{r}_{\mathrm{r}}}\right.$ and $\left.\mathrm{C}_{\mathrm{n}_{\mathrm{r}}}\right)$. These estimates show signs of scattering towards the lower aircraft airspeeds. Plotted against the sideslip angle, $\mathrm{C}_{\mathrm{n}_{\mathrm{r}}}$ shows a tight cluster near or about a zero sideslip value in Figure 6.4.41. 
Figures 6.4.42-6.4.43 represent the aileron rolling and yawing coefficient derivative $\left(\mathrm{C}_{1_{\mathrm{a}_{\mathrm{a}}}}\right.$ and $\left.\mathrm{C}_{\mathrm{n}_{\delta_{\mathrm{a}}}}\right)$, which did not exhibit a loss of effectiveness due to the rolling moment. The adverse yaw effect derivative is normally found to be a negative value, but ideally it would be zero or perhaps slightly positive. The B777 values exhibit both behaviors for various airspeeds in Figure 6.4.42. Figures 6.4.44-6.4.47 represent the rolling and yawing moment estimates due to rudder effectiveness $\left(\mathrm{C}_{\delta_{\mathrm{r}}}\right.$ and $\left.\mathrm{C}_{\mathrm{n}_{\mathrm{r}}}\right)$. Typically the rolling moment due to rudder deflection is positive, however B777 estimates for Flights $\mathrm{A}$ and $\mathrm{C}$ that contain combination maneuvers tended to counteract the rolling moment caused from the rudder, in Figures 6.4.46-6.4.47. Figures 6.4.466.4.47 clearly do show a consistent grouping of the directional control derivative, $\mathrm{C}_{\mathrm{n}_{\delta_{\mathrm{r}}}}$, over the velocity and sideslip ranges.

Figures 6.4.48-6.4.51 represent the sideforce aerodynamic bias and sideslip $\left(\mathrm{C}_{\mathrm{y}_{\mathrm{o}}}\right.$ and $\mathrm{C}_{\mathrm{y}_{\beta}}$ ) estimates. For symmetrical airplanes, the $\mathrm{C}_{\mathrm{y}_{\mathrm{o}}}$ term tends to be equal to zero, this not affected as the airspeed or sideslip increased. The $\mathrm{C}_{\mathrm{y} \beta}$ term represents the derivative describing the dutch-roll dynamics for the aircraft. Typically a negative value, $\mathrm{C}_{\mathrm{y} \beta}$ was found to be opposite in sign but with very large CRB values at high airspeed and positive average sideslip. This indicated a less then favorable representation of the contribution of wing and vertical tail. Figures 6.4.52-6.4.55 represent the sideforce for rolling and yawing moments $\left(\mathrm{C}_{\mathrm{y}_{\mathrm{p}}}\right.$ and $\left.\mathrm{C}_{\mathrm{y}_{\mathrm{r}}}\right)$. Figures 6.4 .56 to 6.4 .59 represent the sideforce estimates due to aileron and rudder effectiveness $\left(\mathrm{C}_{\mathrm{y} \delta_{\mathrm{a}}}\right.$ and $\left.\mathrm{C}_{\mathrm{y} \delta_{\mathrm{r}}}\right)$. The $\mathrm{C}_{\mathrm{y} \delta_{\mathrm{a}}}$ value can normally be considered negligible, but plots indicate large CRB, or unclear impression of the estimates at higher airspeeds. This could be cause due to a close proximity of the aircraft's fuselage, elevator and vertical tail. The $\mathrm{C}_{\mathrm{y}} \delta_{\mathrm{r}}$, seen in Figure 6.4.59estimates showed excellent consistency, with a tendency to slighty decrease as sideslip increased. 
Flight C, Full Flight Time History
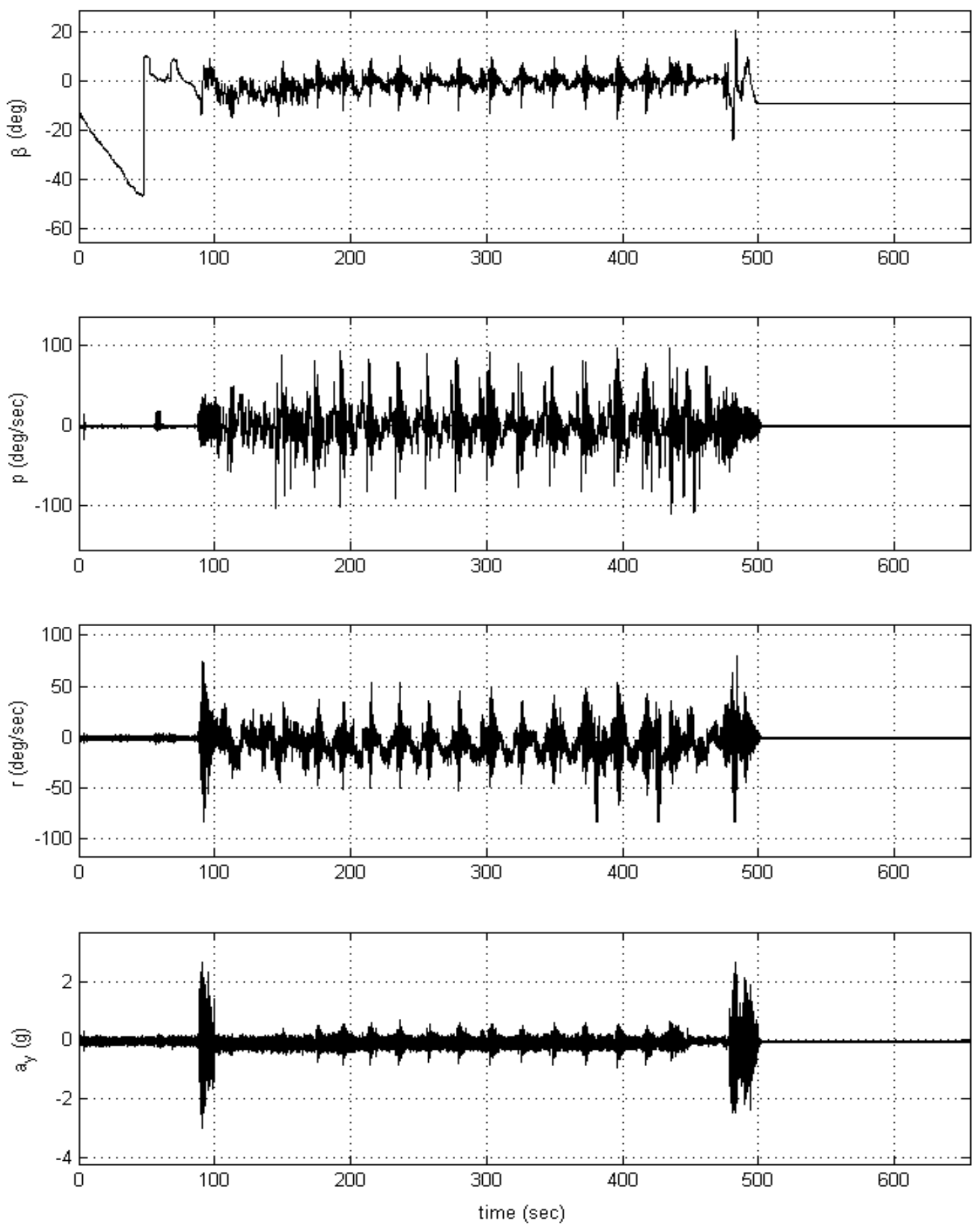

Figure 6.4.1 Sample time history of aircraft parameters for lateral-directional Flight $\mathrm{C}$ ( $\beta$, roll rate, yaw rate, lateral acceleration) 
Flight C, Full Flight Time History
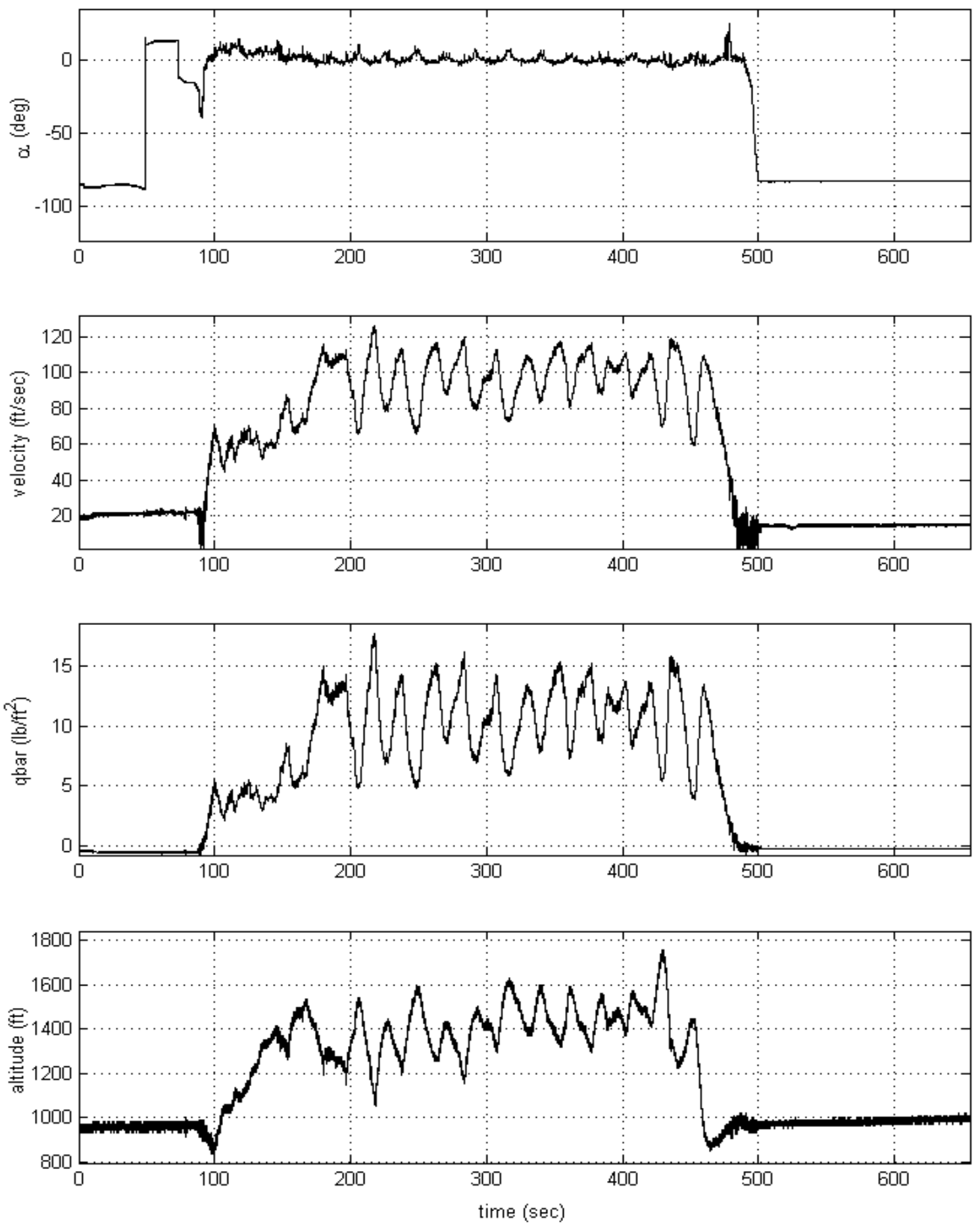

Figure 6.4.2 Sample time history of aircraft parameters for lateral-directional Flight C $(\alpha$, velocity, dynamic pressure, altitude) 
Flight C, Full Flight Time History
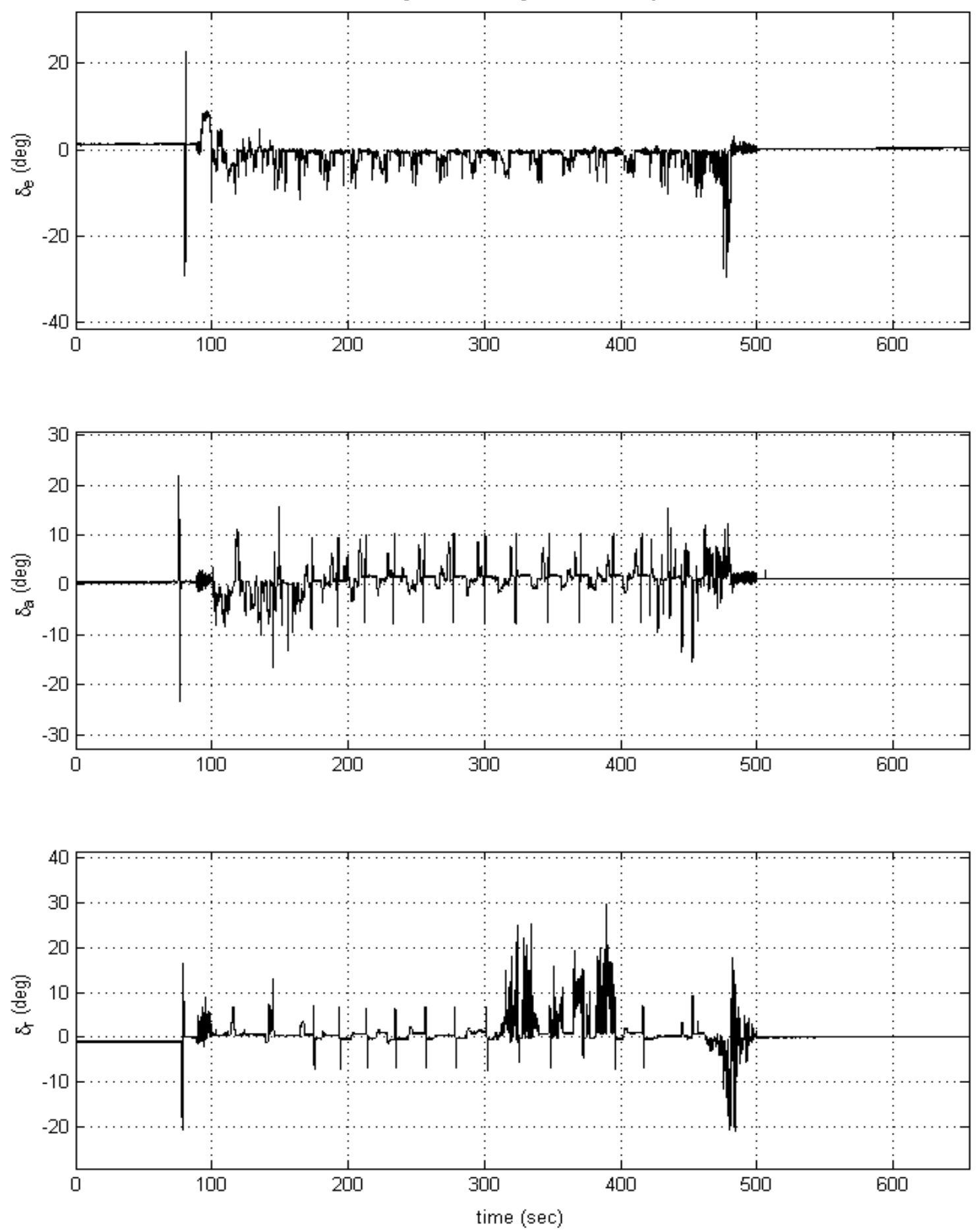

Figure 6.4.3 Sample time history of aircraft parameters for lateral-directional Flight C $\left(\delta_{\mathrm{e}}, \delta_{\mathrm{a}}, \delta_{\mathrm{r}}\right)$ 
Flight A, Maneuver 8 (lateral-directional input)
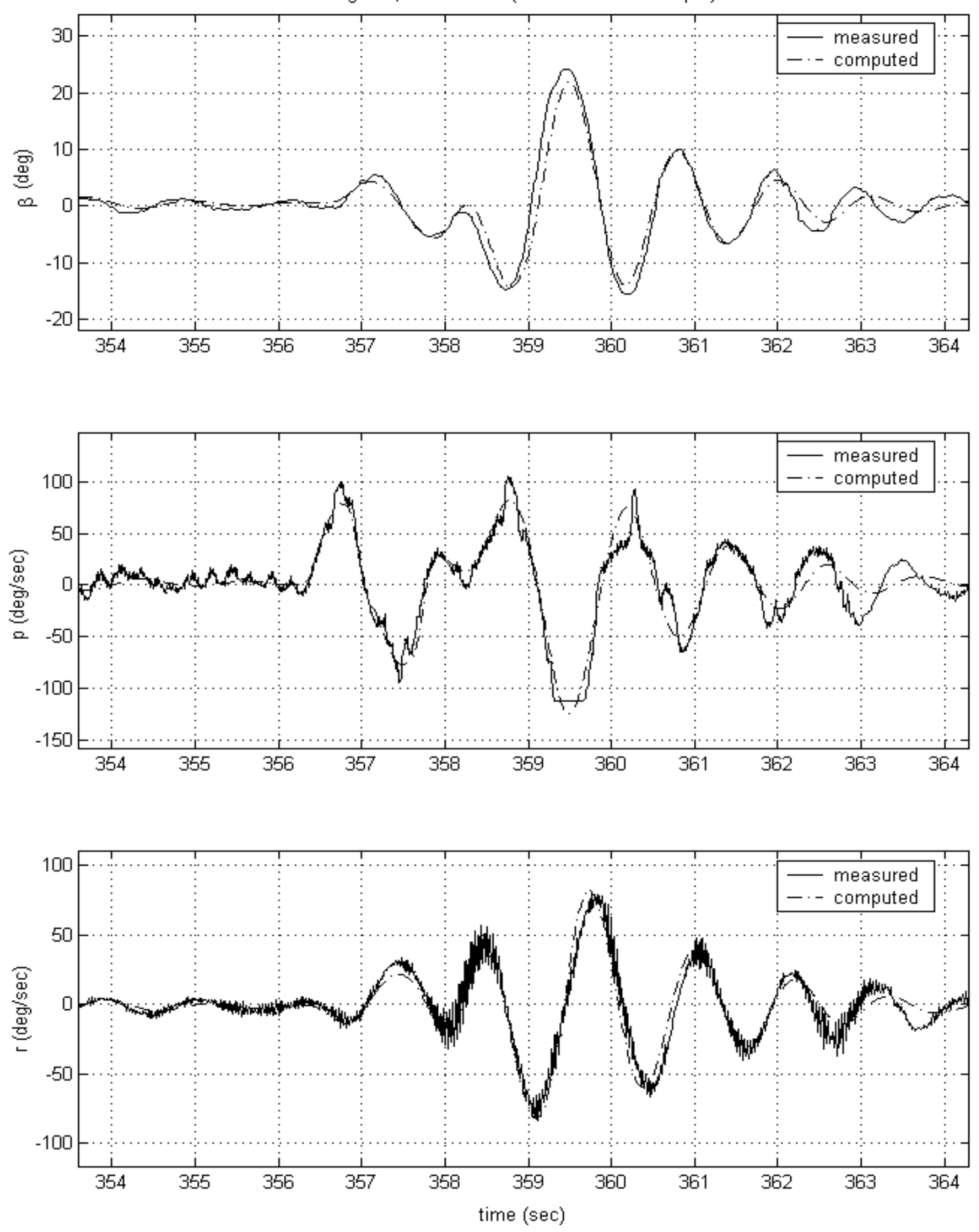

Figure 6.4.4 Comparison of measured and computed time histories from Flight A, Maneuver 8, lateral-directional input ( $\beta$, roll rate, yaw rate) 
Flight A, Maneuver 8 (lateral-directional input)
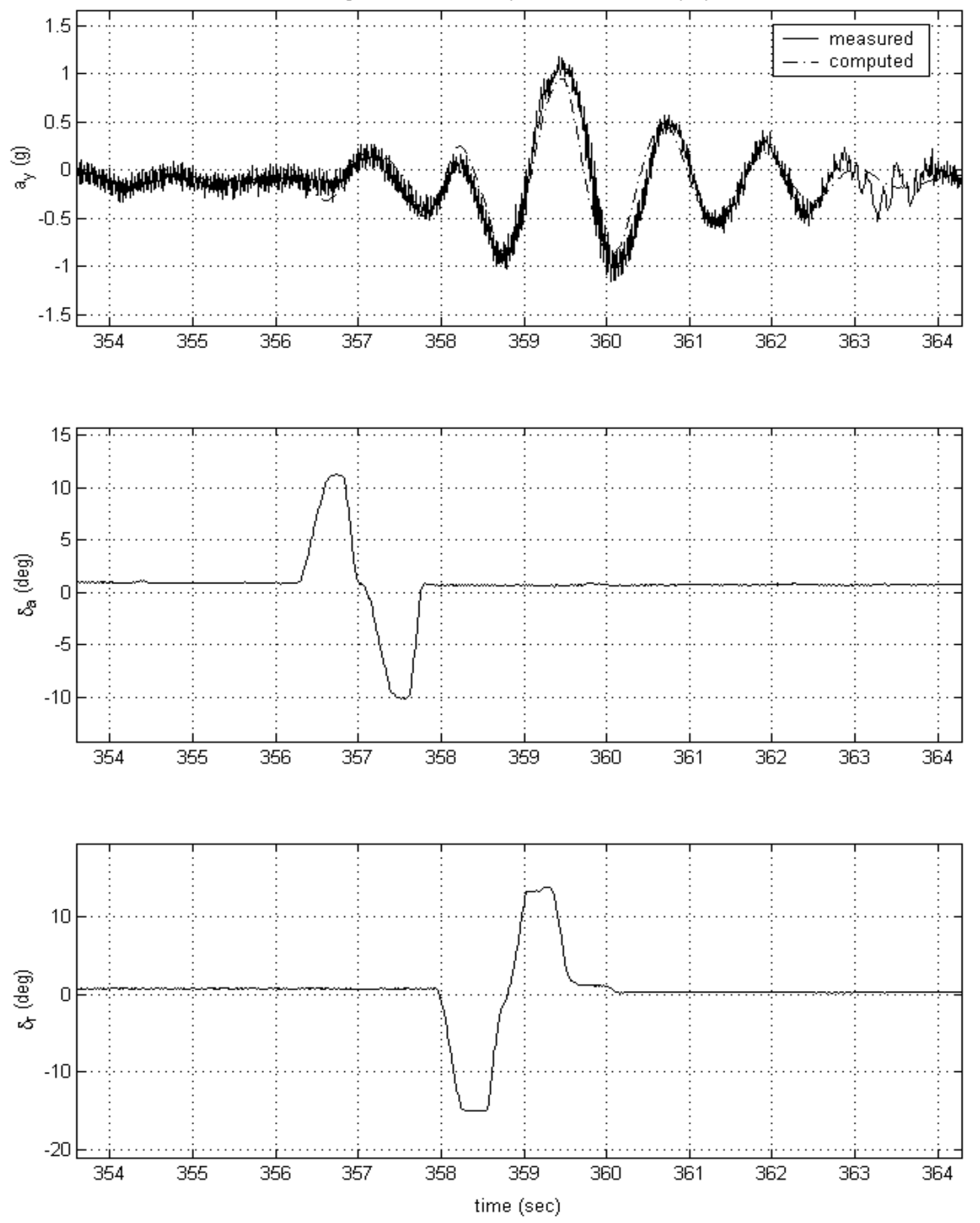

Figure 6.4.5 Comparison of measured and computed time histories from Flight A, Maneuver 8, lateral-directional input (lateral acceleration, $\delta_{\mathrm{a}}, \delta_{\mathrm{r}}$ ) 
Flight A, Maneuver 8 (lateral-directional input)
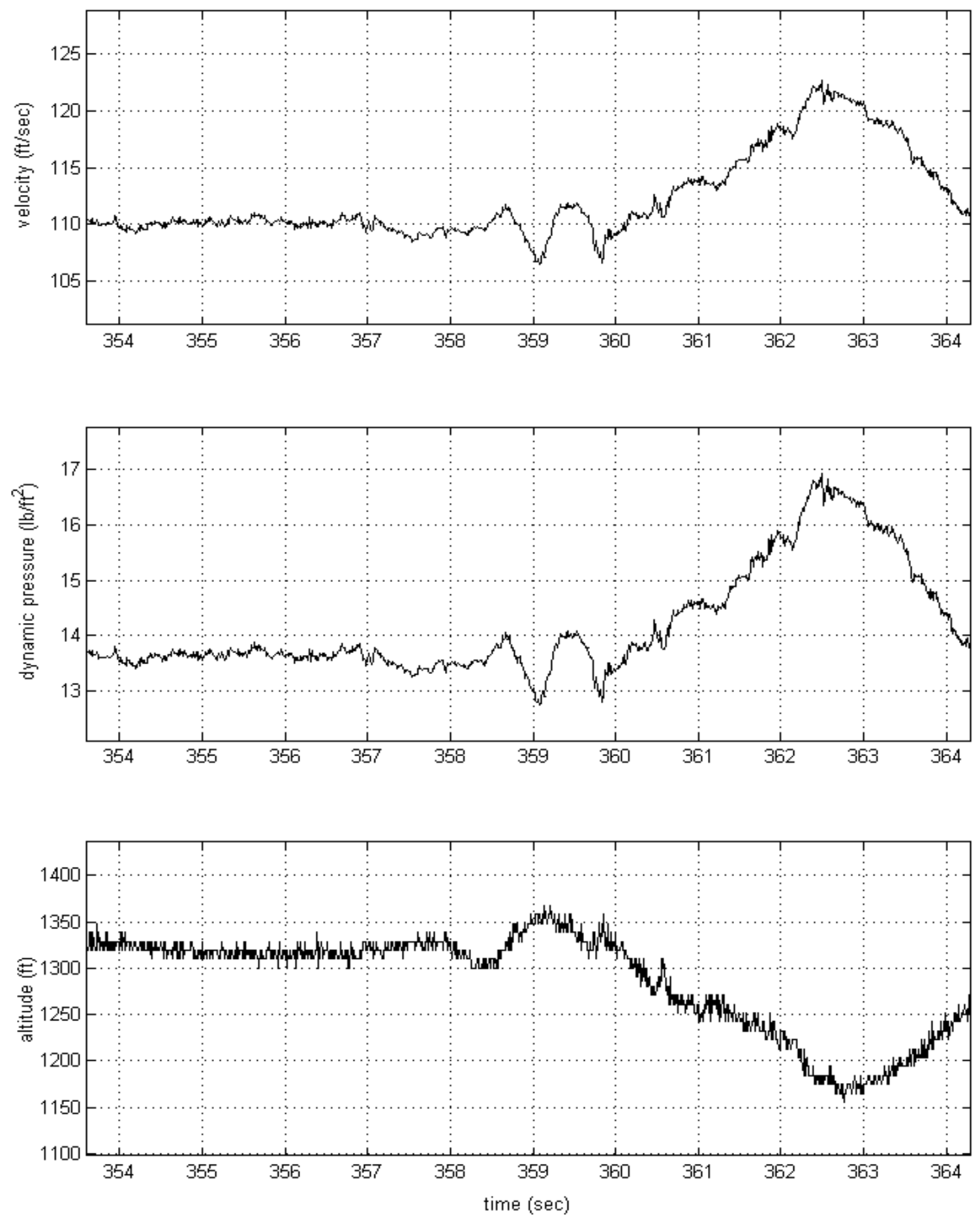

Figure 6.4.6 Comparison of measured and computed time histories from Flight A, Maneuver 8, lateral-directional input (velocity, dynamic pressure, altitude) 
Flight B, Maneuver 8 (lateral-directional input)
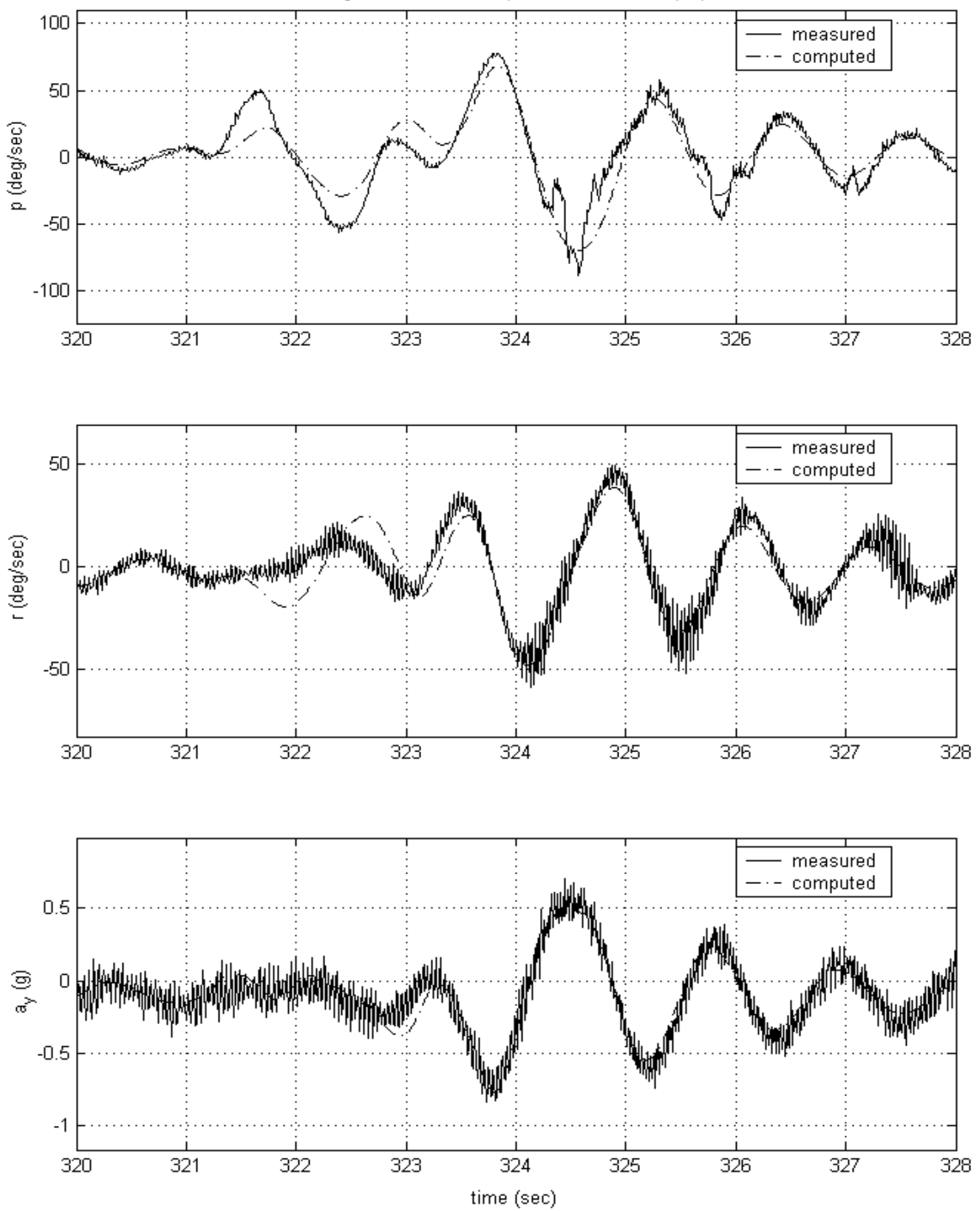

Figure 6.4.7 Comparison of measured and computed time histories from Flight B, Maneuver 8, lateral-directional input (roll rate, yaw rate, lateral acceleration) 
Flight B, Maneuver 8 (lateral-directional input)
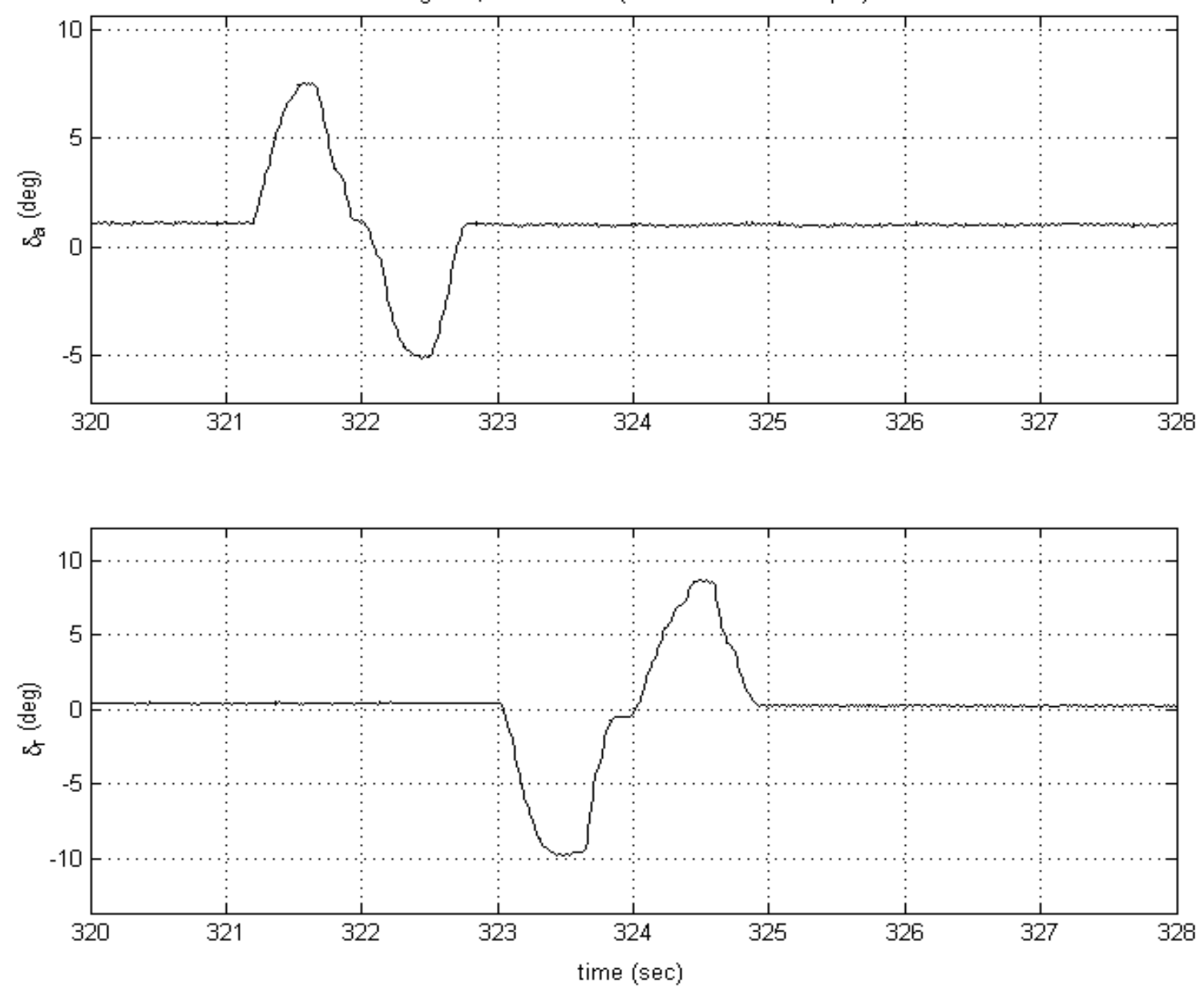

Figure 6.4.8 Comparison of measured and computed time histories from Flight B, Maneuver 8, lateral-directional input $\left(\delta_{\mathrm{a}}, \delta_{\mathrm{r}}\right)$ 
Flight $\mathrm{C}$, Maneuver 7 (lateral-directional input)
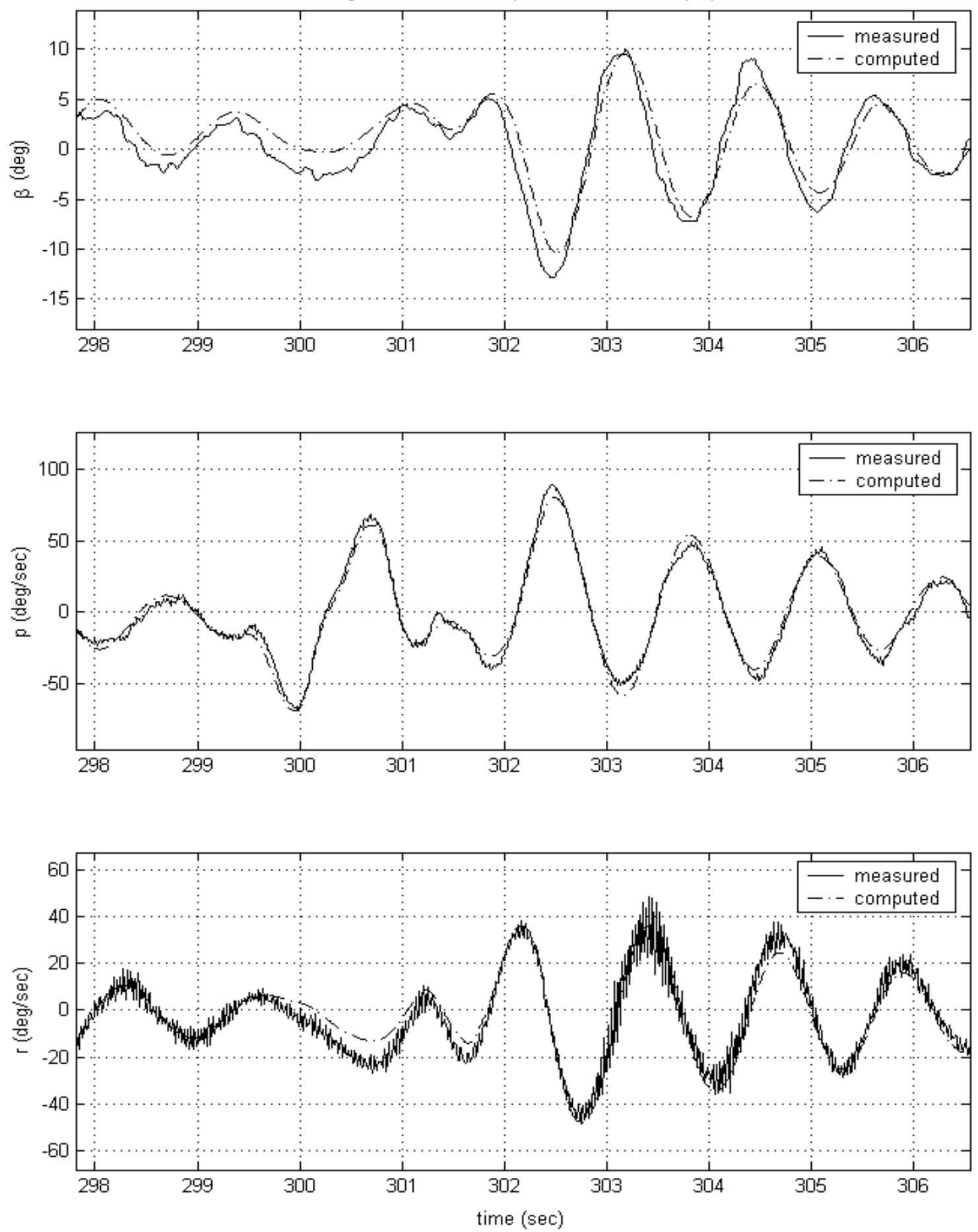

Figure 6.4.9 Comparison of measured and computed time histories from Flight $C$, Maneuver 7, lateral-directional input ( $\beta$, roll rate, yaw rate) 
Flight $\mathrm{C}$, Maneuver 7 (lateral-directional input)
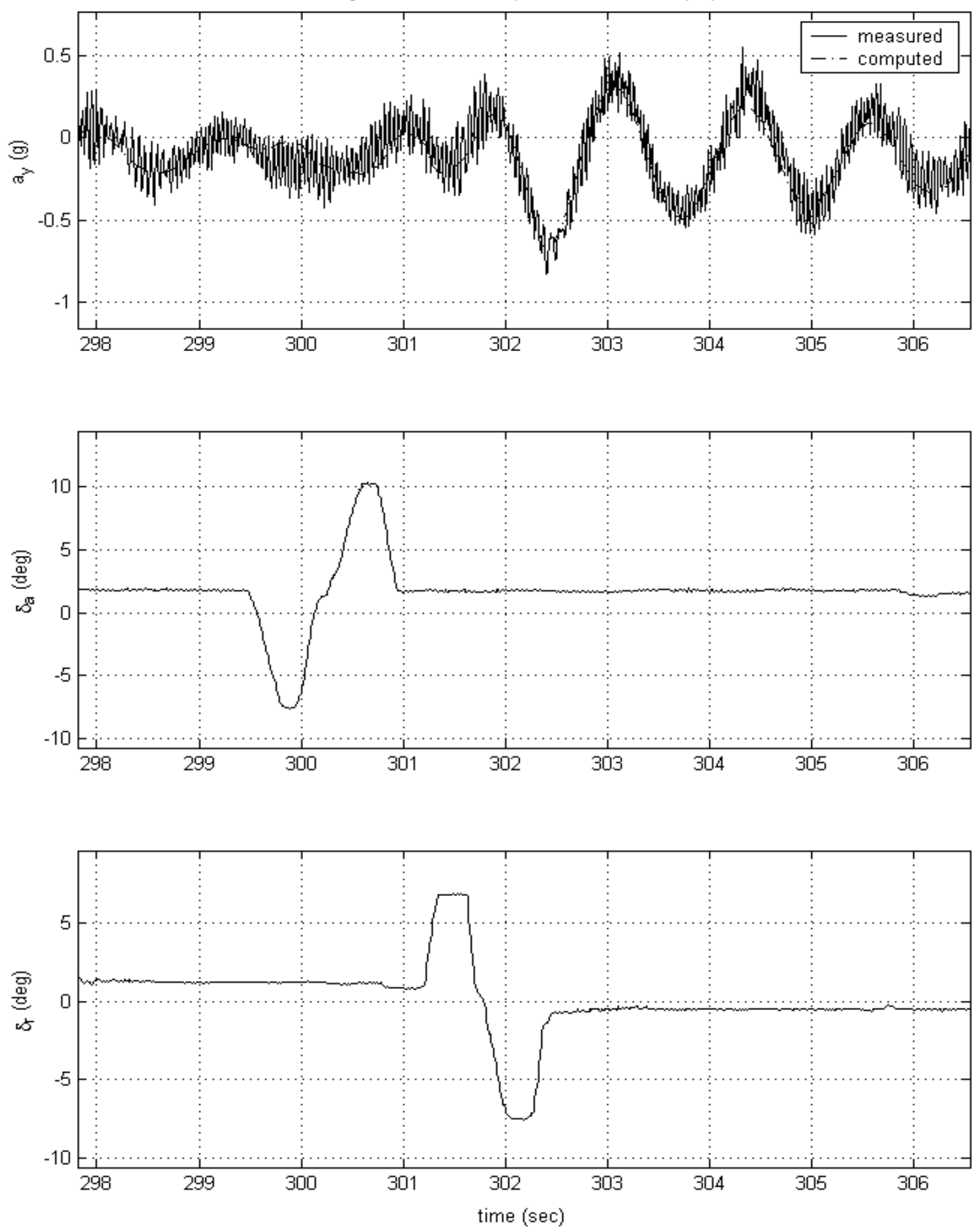

Figure 6.4.10 Comparison of measured and computed time histories from Flight $C$, Maneuver 7, lateral-directional input (lateral acceleration, $\delta_{\mathrm{a}}, \delta_{\mathrm{r}}$ ) 
Flight $\mathrm{C}$, Maneuver 7 (lateral-directional input)
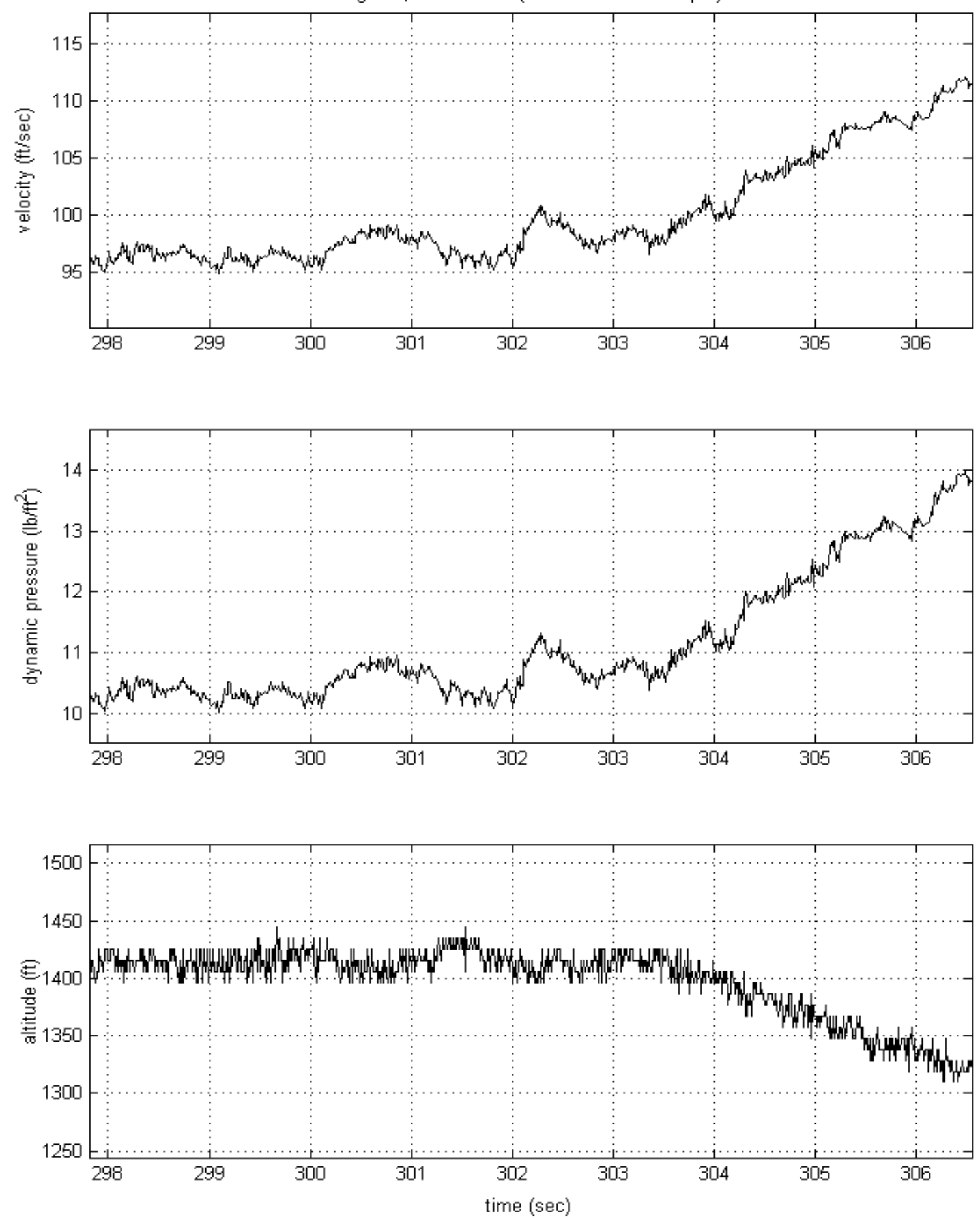

Figure 6.4.11 Comparison of measured and computed time histories from Flight $\mathrm{C}$, Maneuver 7, lateral-directional input (velocity, dynamic pressure, altitude) 
Flight D, Maneuver 6 (lateral-directional input)
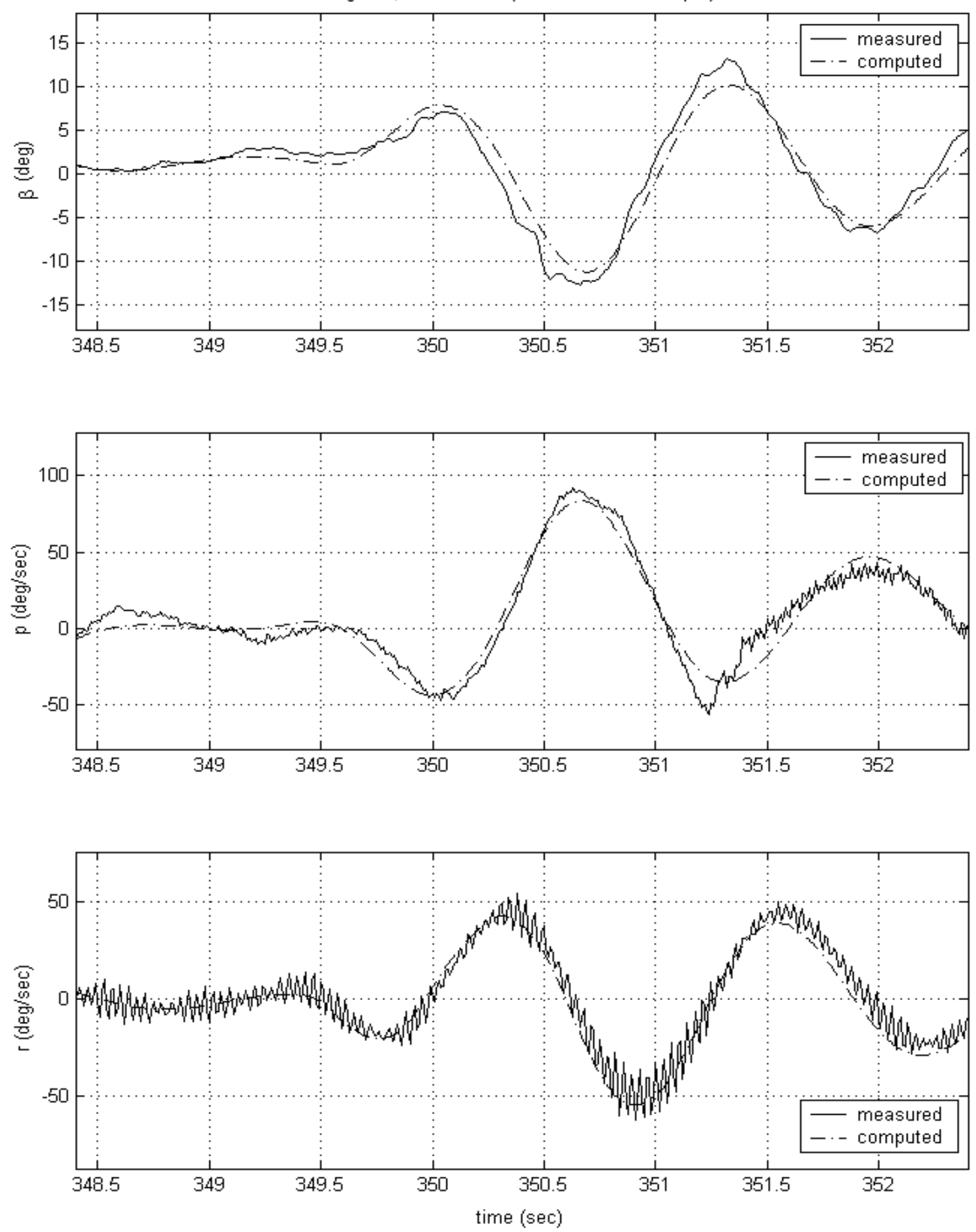

Figure 6.4.12 Comparison of measured and computed time histories from Flight D, Maneuver 6, lateral-directional input ( $\beta$, roll rate, yaw rate) 
Flight D, Maneuver 6 (lateral-directional input)
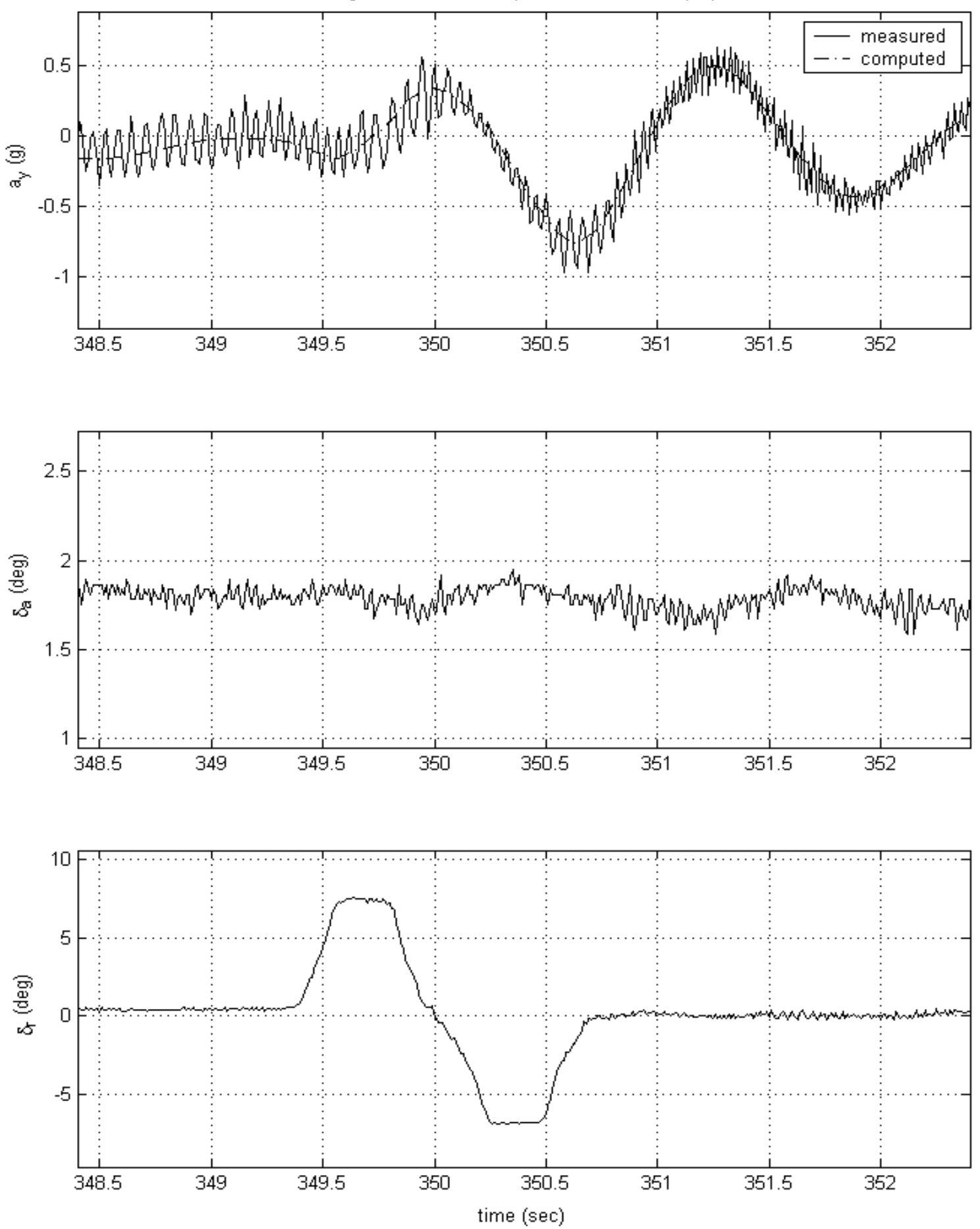

Figure 6.4.13 Comparison of measured and computed time histories from Flight $D$, Maneuver 6, lateral-directional input (lateral acceleration, $\delta_{\mathrm{a}}, \delta_{\mathrm{r}}$ ) 
Flight D, Maneuver 6 (lateral-directional input)
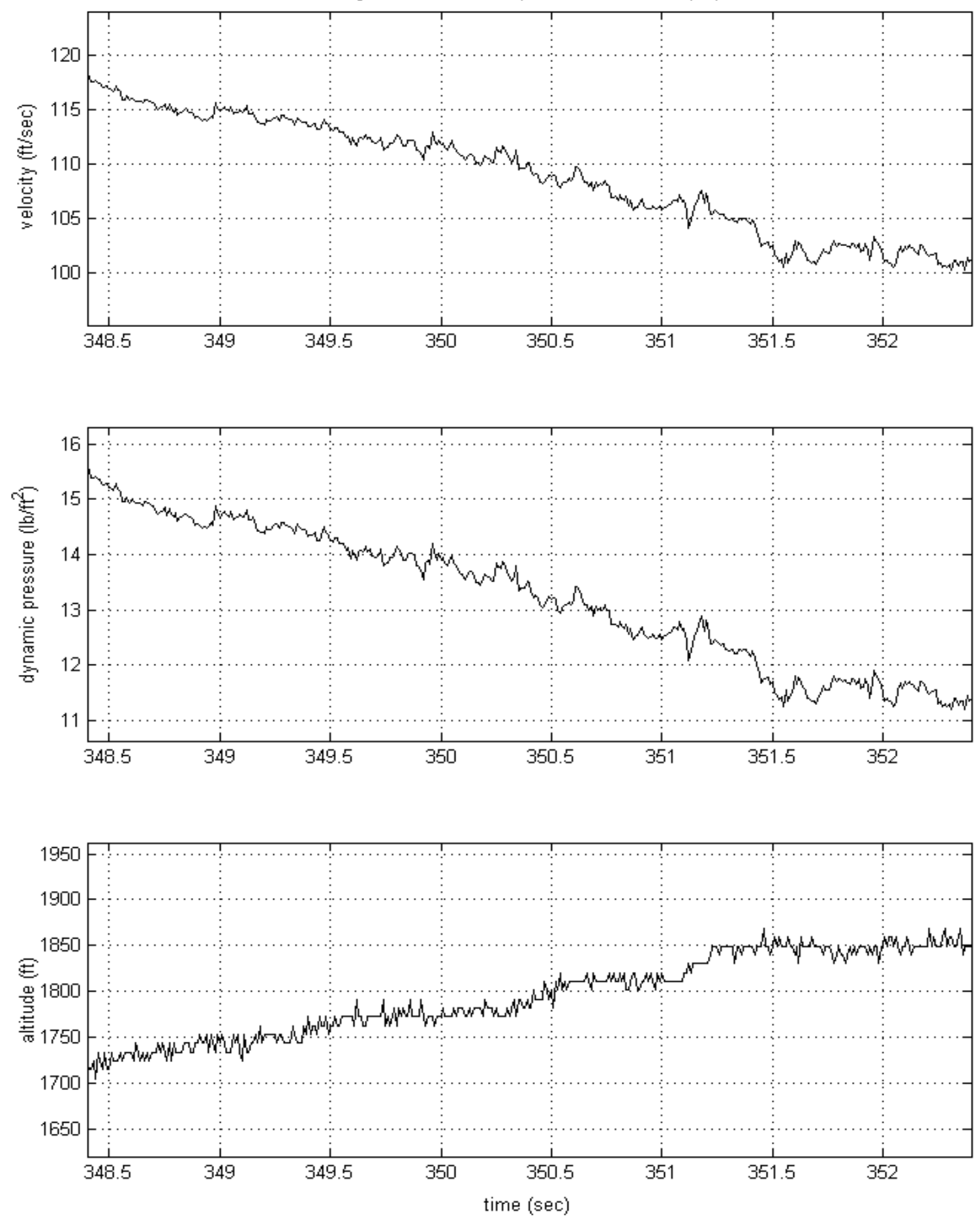

Figure 6.4.14 Comparison of measured and computed time histories from Flight D, Maneuver 6, lateral-directional input (velocity, dynamic pressure altitude) 
Flight E, Maneuver 10 (lateral-directional input)
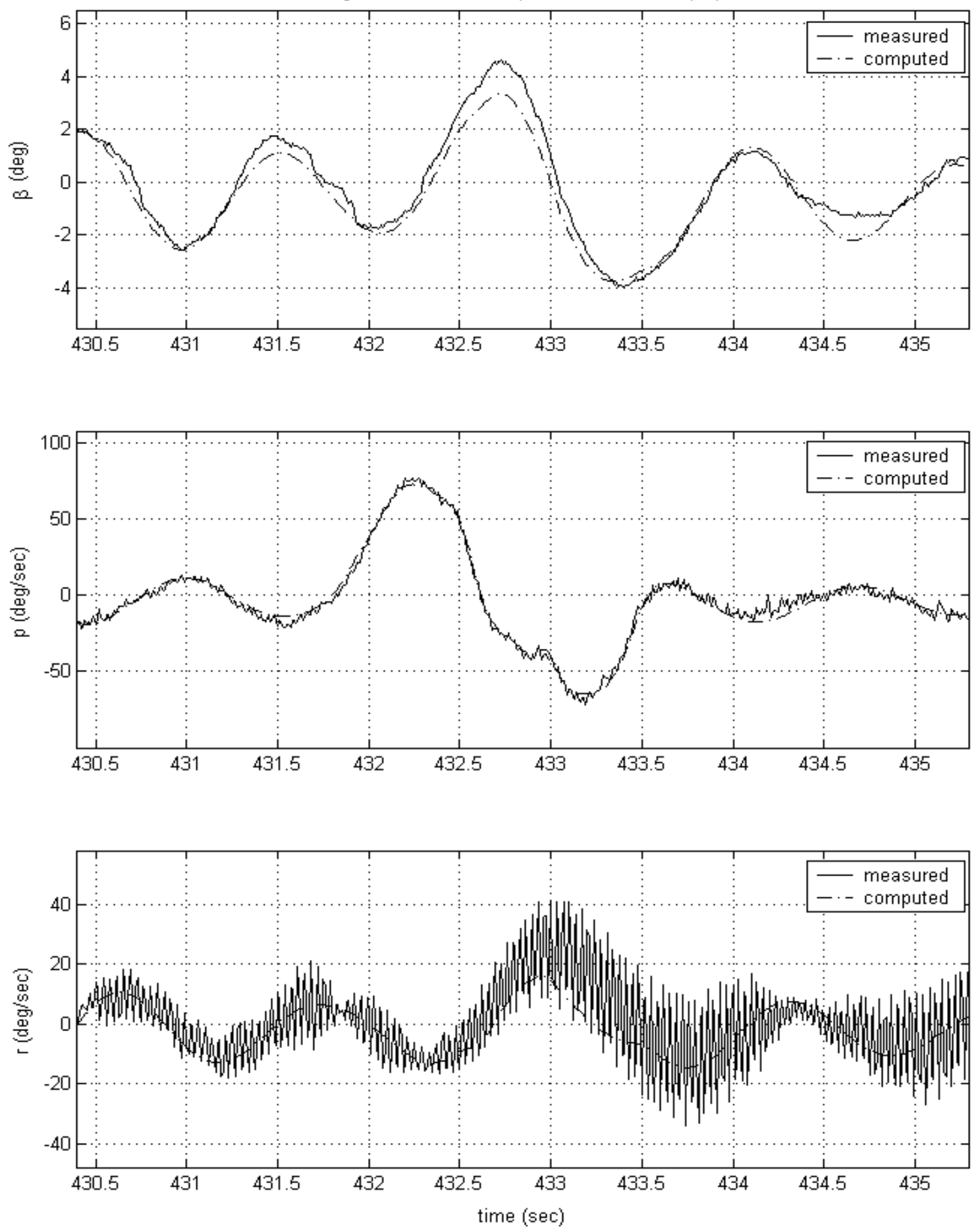

Figure 6.4.15 Comparison of measured and computed time histories from Flight E, Maneuver 10, lateral-directional input ( $\beta$, roll rate, yaw rate) 
Flight E, Maneuver 10 (lateral-directional input)
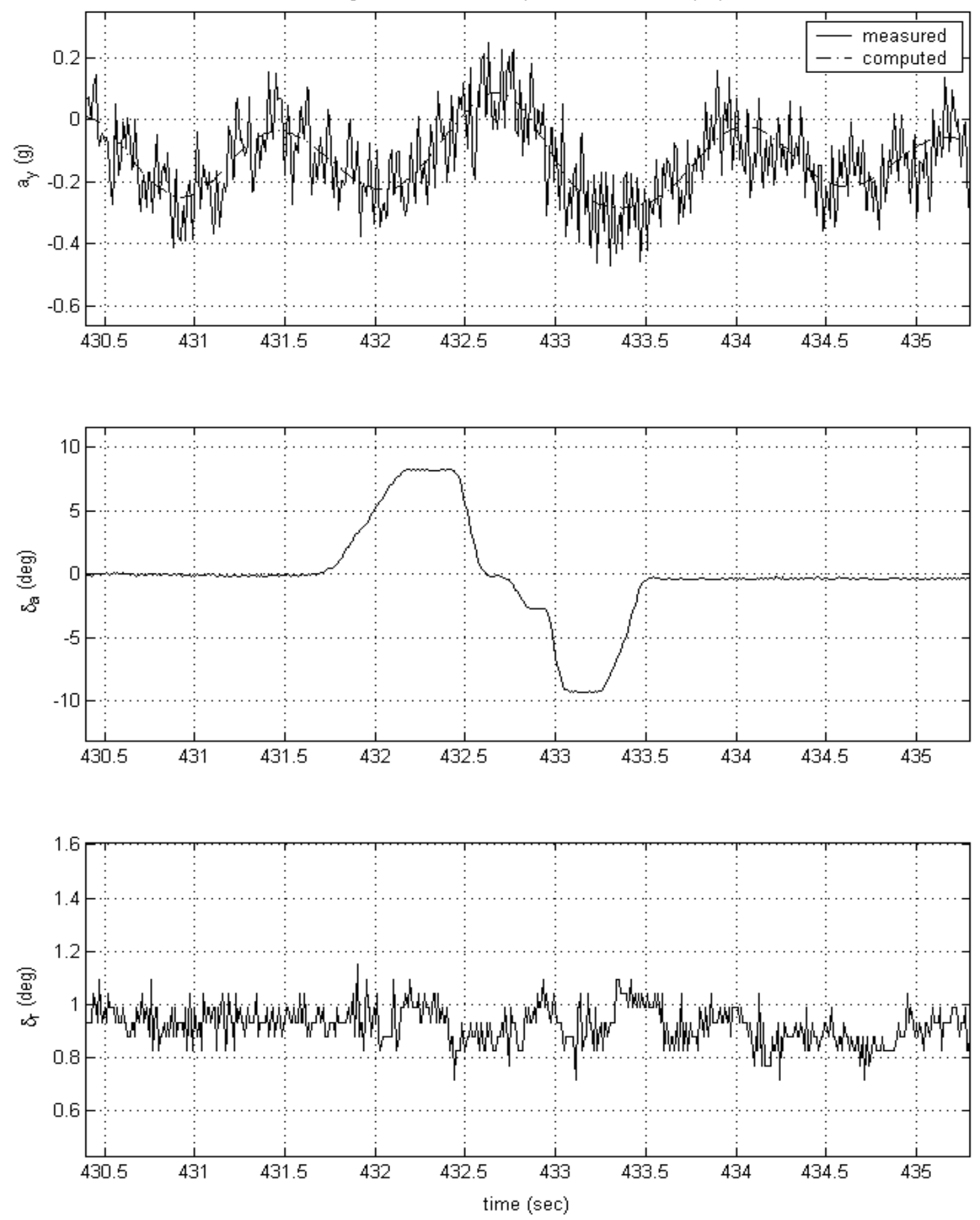

Figure 6.4.16 Comparison of measured and computed time histories from Flight E, Maneuver 10, lateral-directional input (lateral acceleration, $\delta_{\mathrm{a}}, \delta_{\mathrm{r}}$ ) 
Flight E, Maneuver 10 (lateral-directional input)
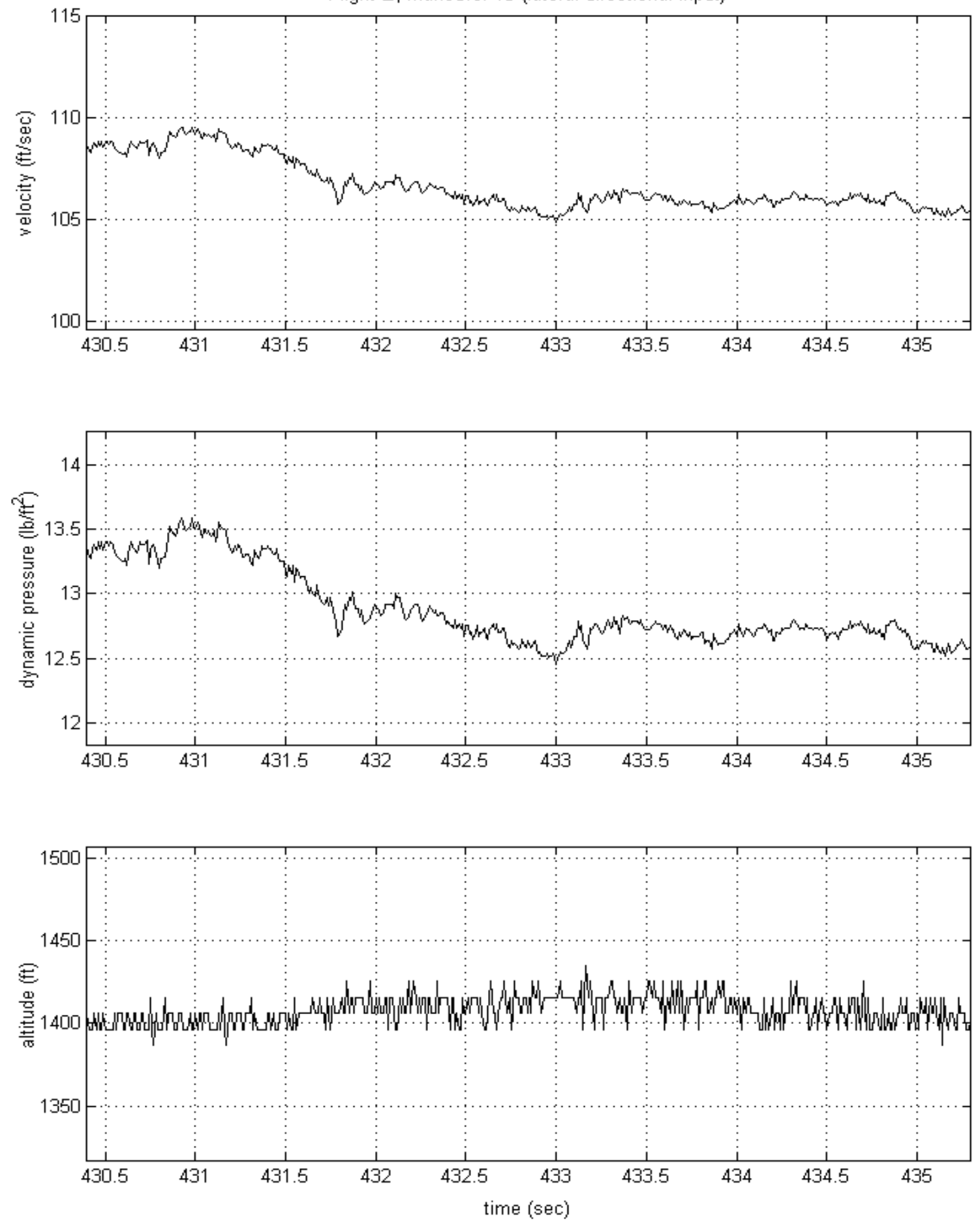

Figure 6.4.17 Comparison of measured and computed time histories from Flight E, Maneuver 10, lateral-directional input (velocity, dynamic pressure, altitude) 

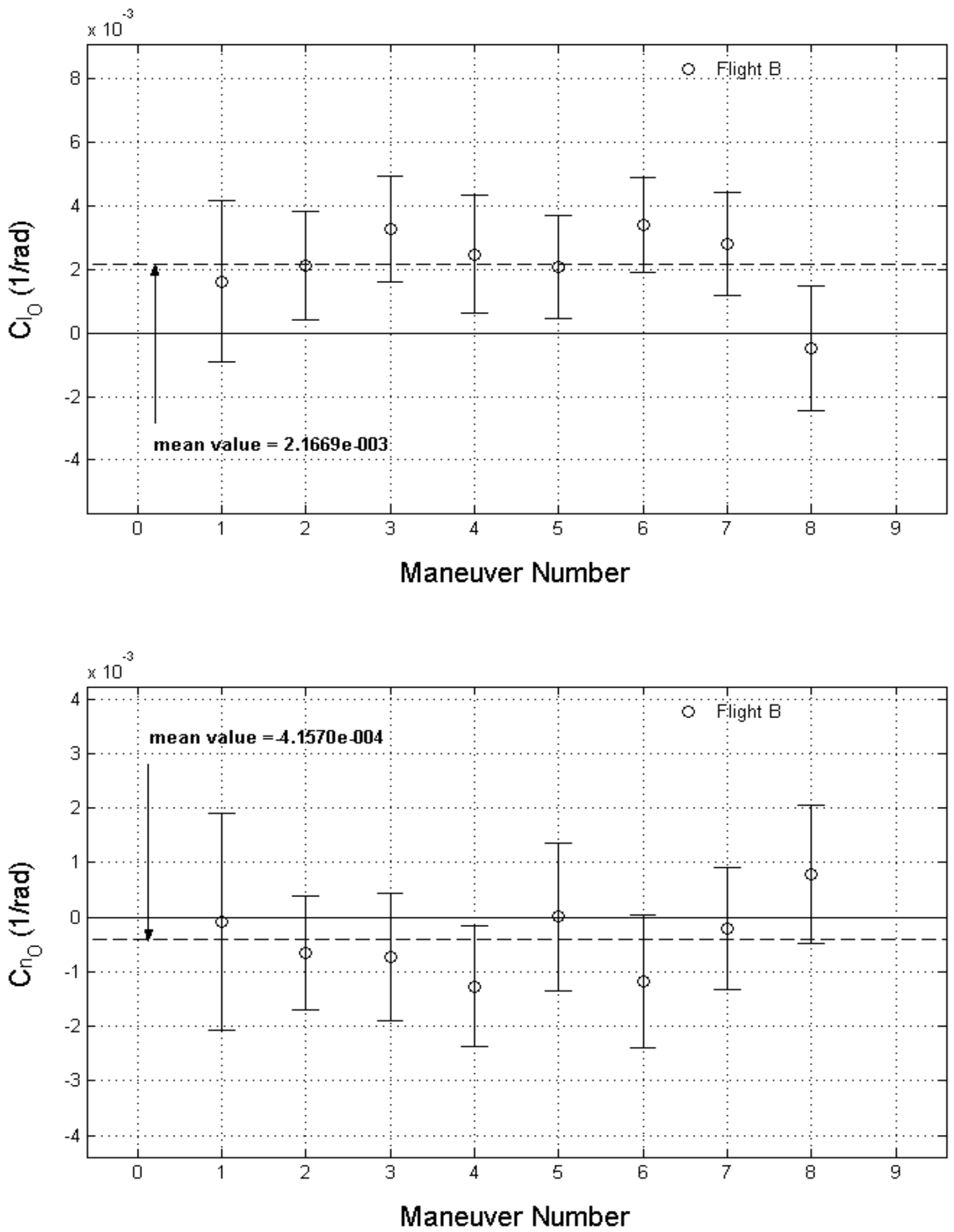

Figure 6.4.18 Flight $B$ maneuvers vs. $C_{l_{O}}$ and $C_{n_{o}}$ off-line estimation results 

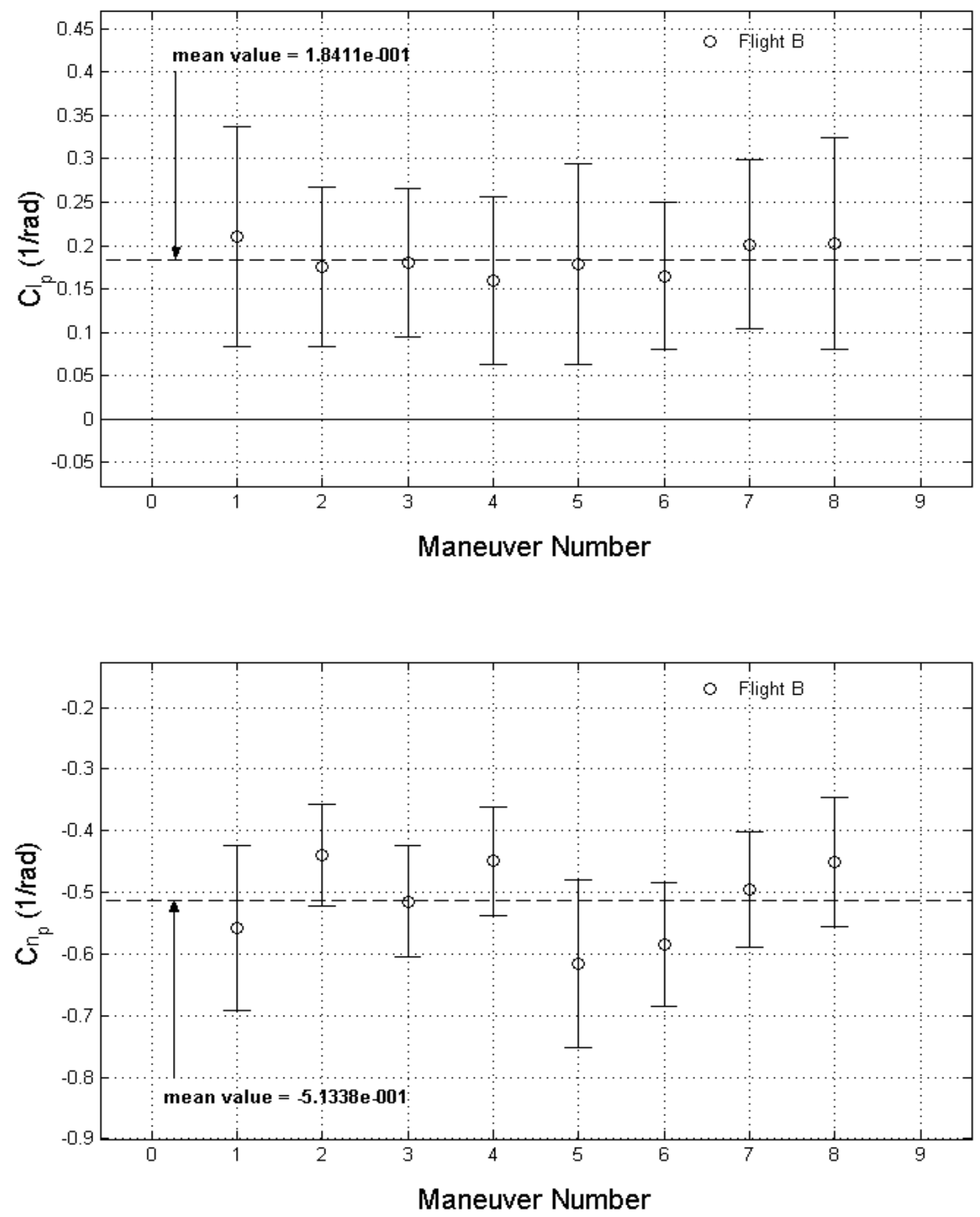

Figure 6.4.19 Flight $B$ maneuvers vs. $C_{l_{p}}$ and $C_{n_{p}}$ off-line estimation results 

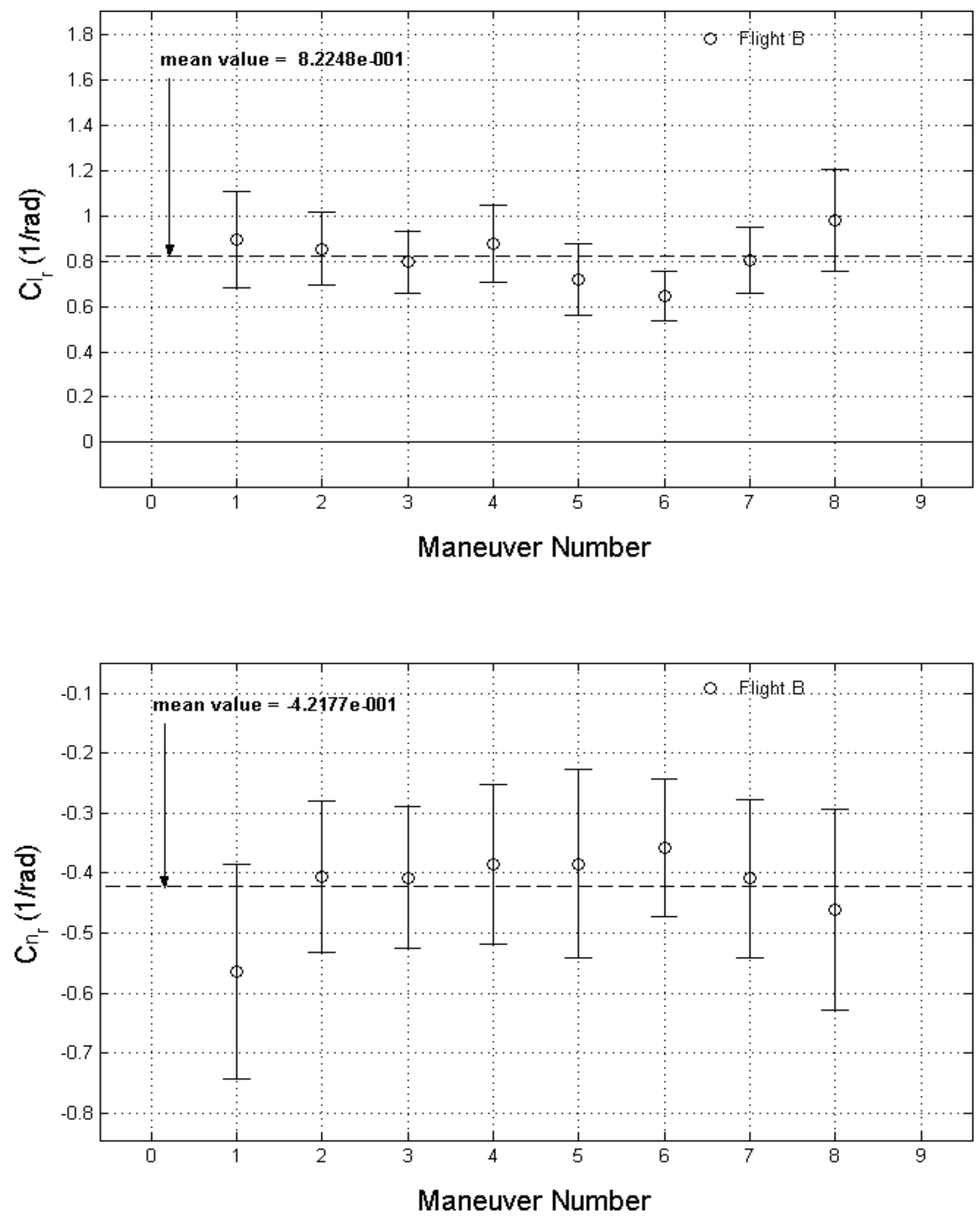

Figure 6.4.20 Flight $B$ maneuvers vs. $\mathrm{C}_{\mathrm{r}_{\mathrm{r}}}$ and $\mathrm{C}_{\mathrm{n}_{\mathrm{r}}}$ off-line estimation results 

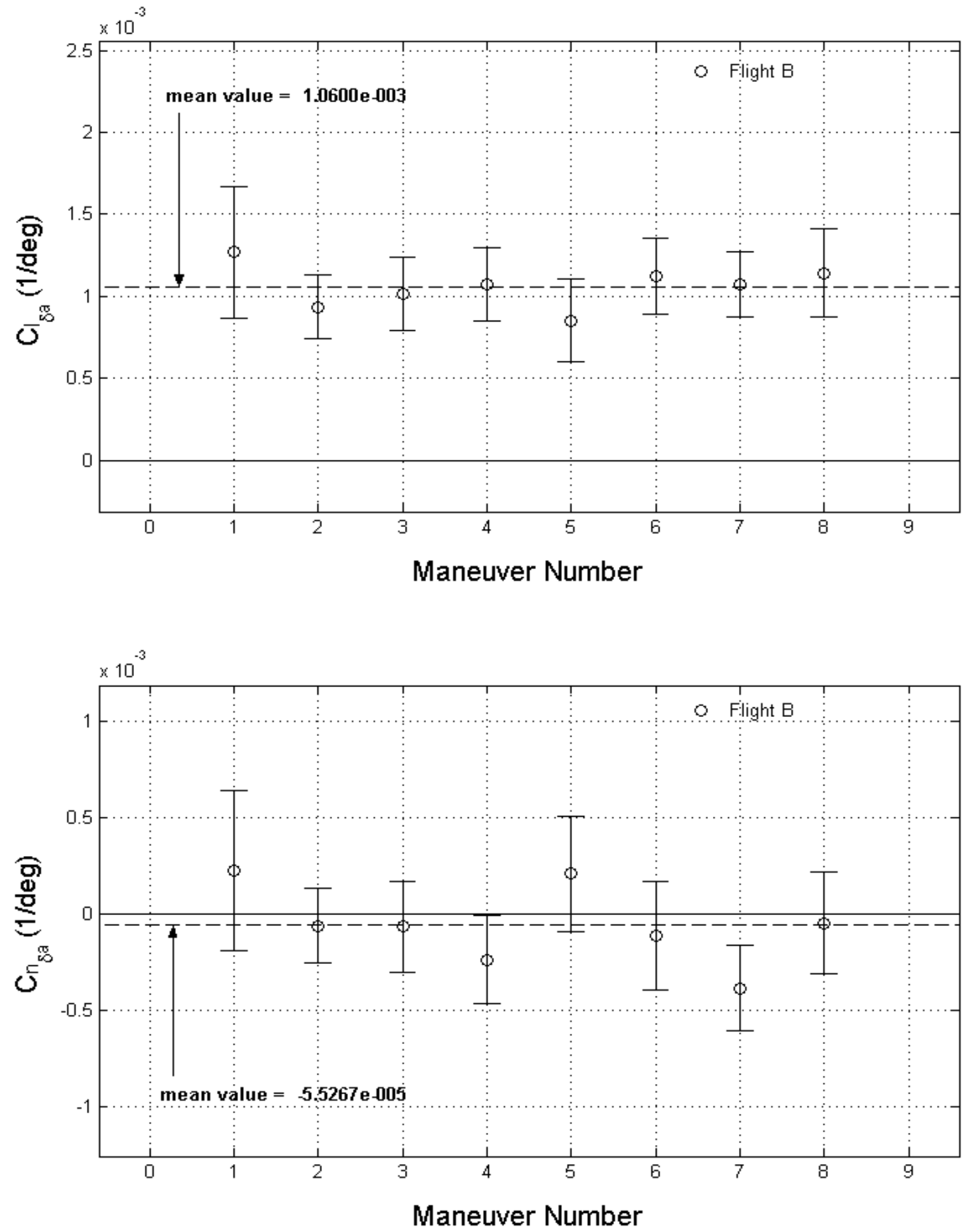

Figure 6.4.21 Flight B maneuvers vs. $\mathrm{C}_{\delta_{\mathrm{a}}}$ and $\mathrm{C}_{\mathrm{n}_{\mathrm{a}}}$ off-line estimation results 

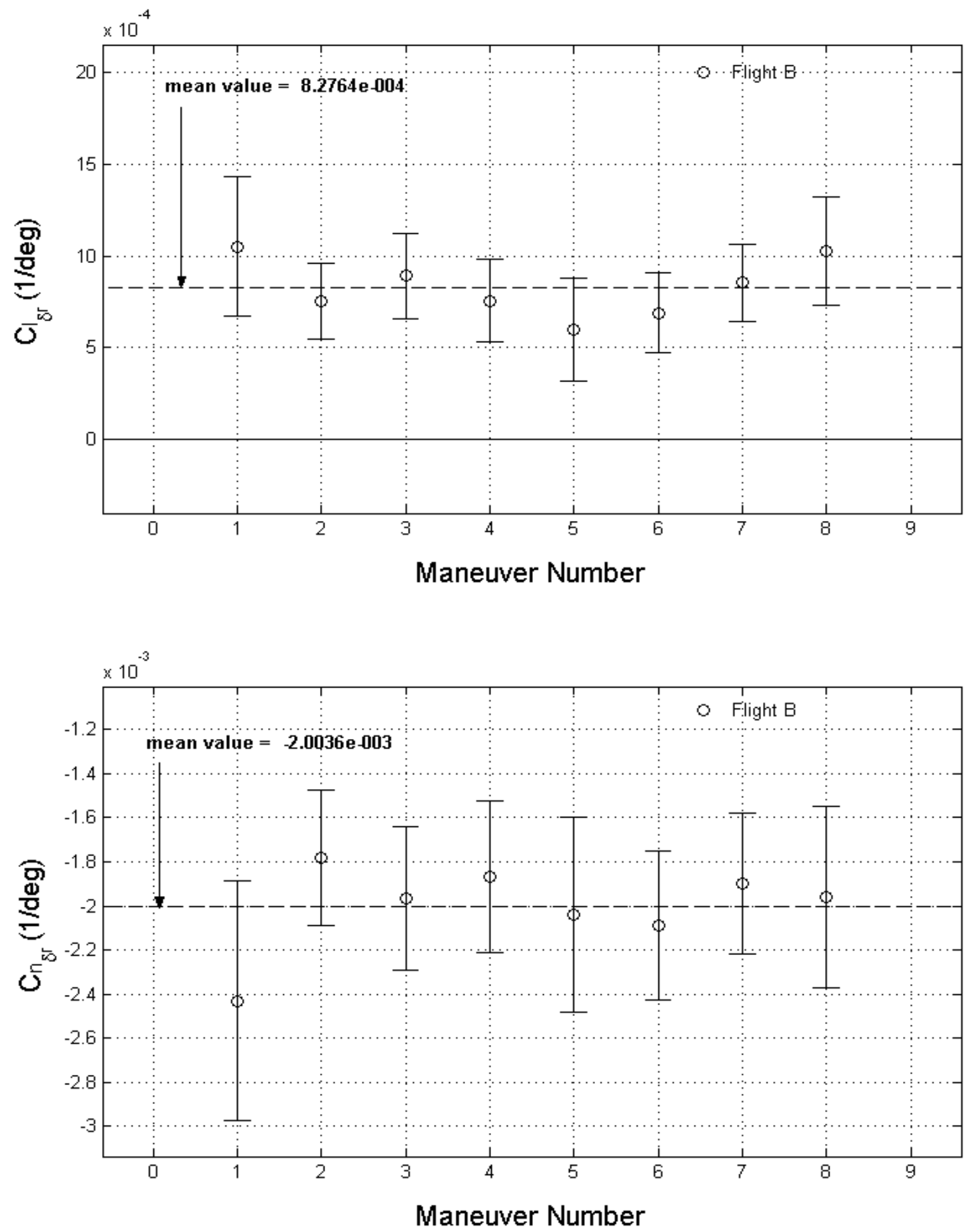

Figure 6.4.22 Flight B maneuvers vs. $\mathrm{C}_{1_{\mathrm{r}_{\mathrm{r}}}}$ and $\mathrm{C}_{\mathrm{n}_{\delta_{\mathrm{r}}}}$ off-line estimation results 

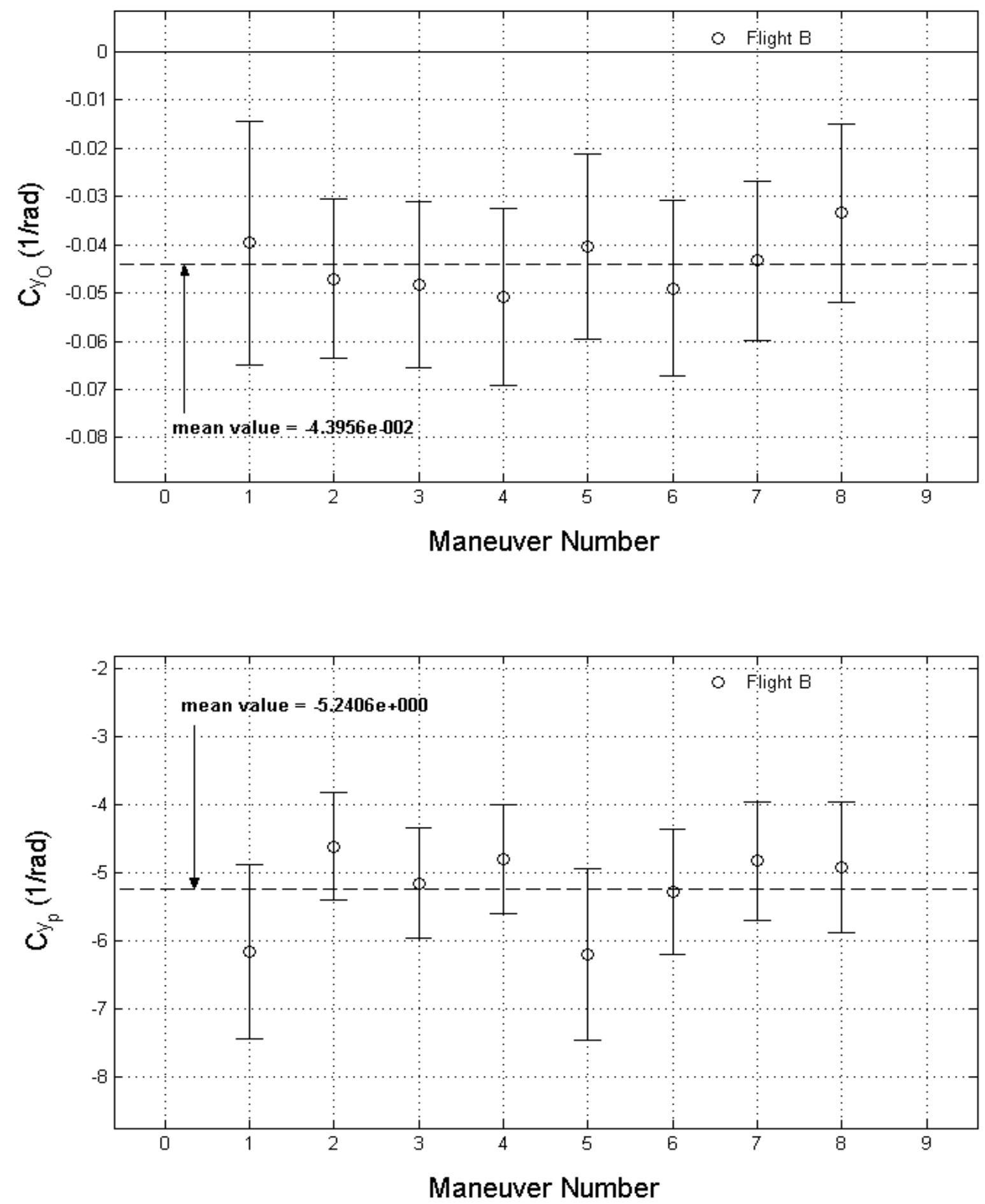

Figure 6.4.23 Flight $B$ maneuvers vs. $C_{y_{o}}$ and $C_{y_{p}}$ off-line estimation results 

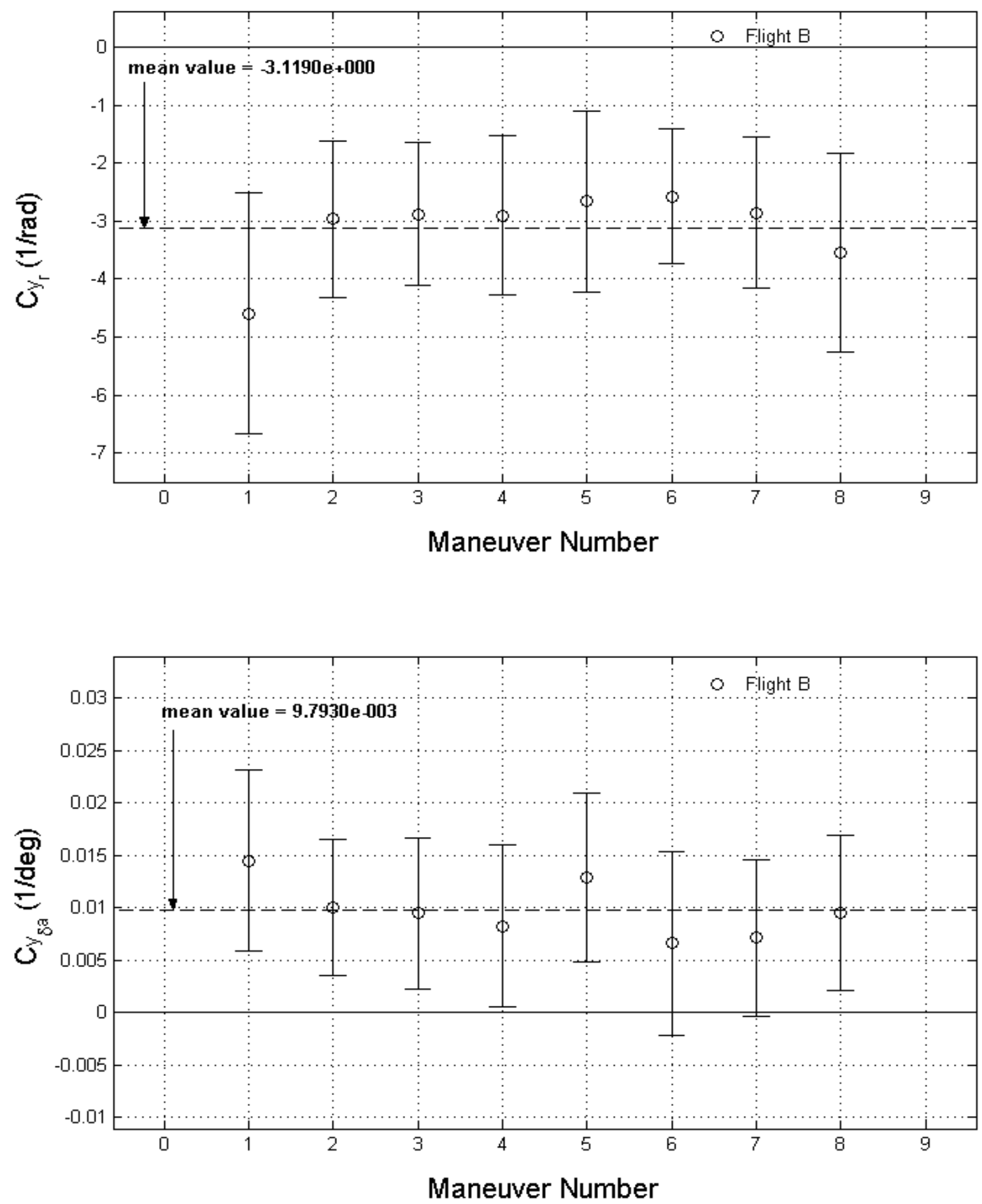

Figure 6.4.24 Flight $\mathrm{B}$ maneuvers vs. $\mathrm{C}_{\mathrm{y}_{\mathrm{r}}}$ and $\mathrm{C}_{\mathrm{y}_{\delta_{\mathrm{a}}}}$ off-line estimation results 


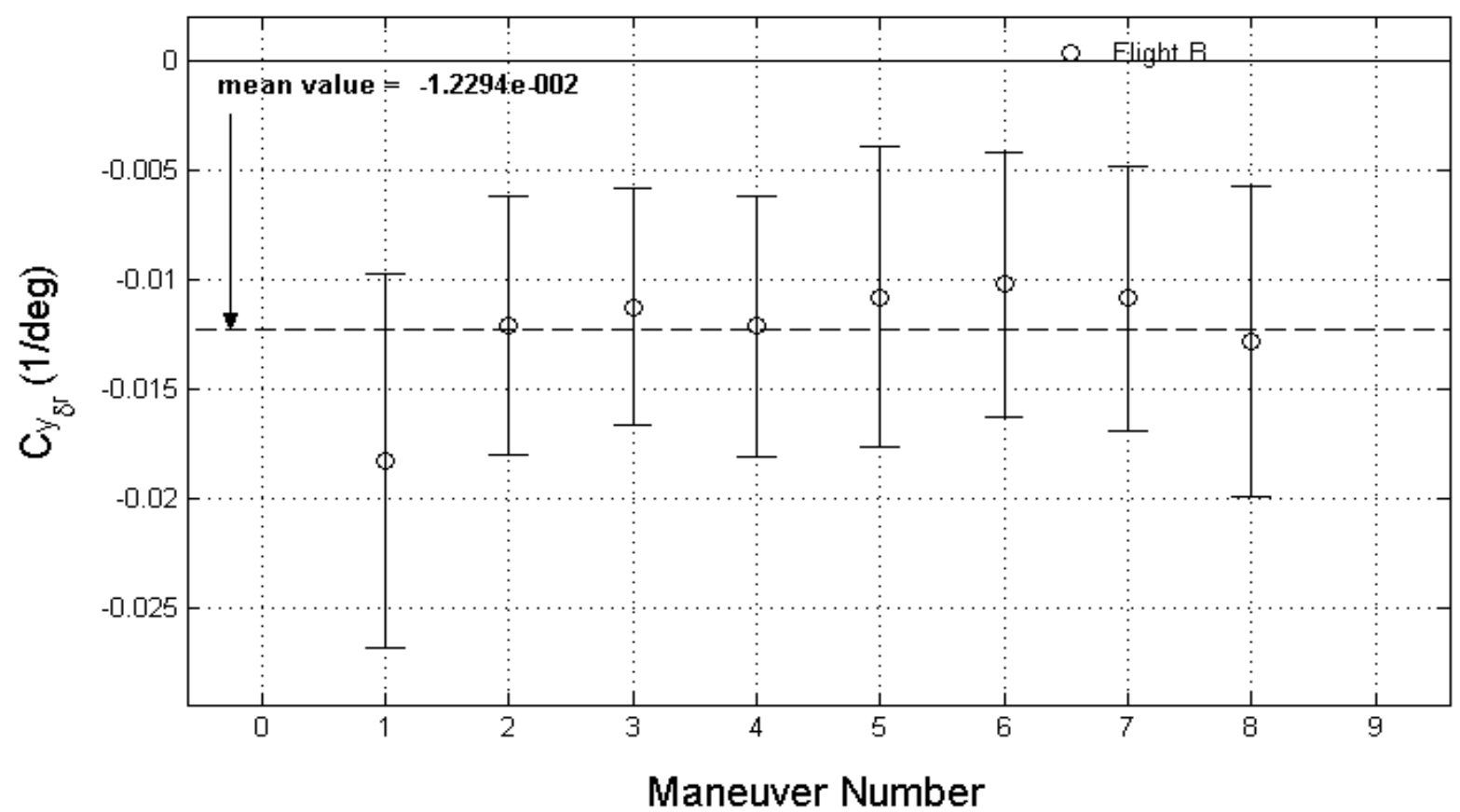

Figure 6.4.25 Flight $\mathrm{B}$ maneuvers vs. $\mathrm{C}_{\mathrm{y}_{\delta_{\mathrm{r}}}}$ off-line estimation results 

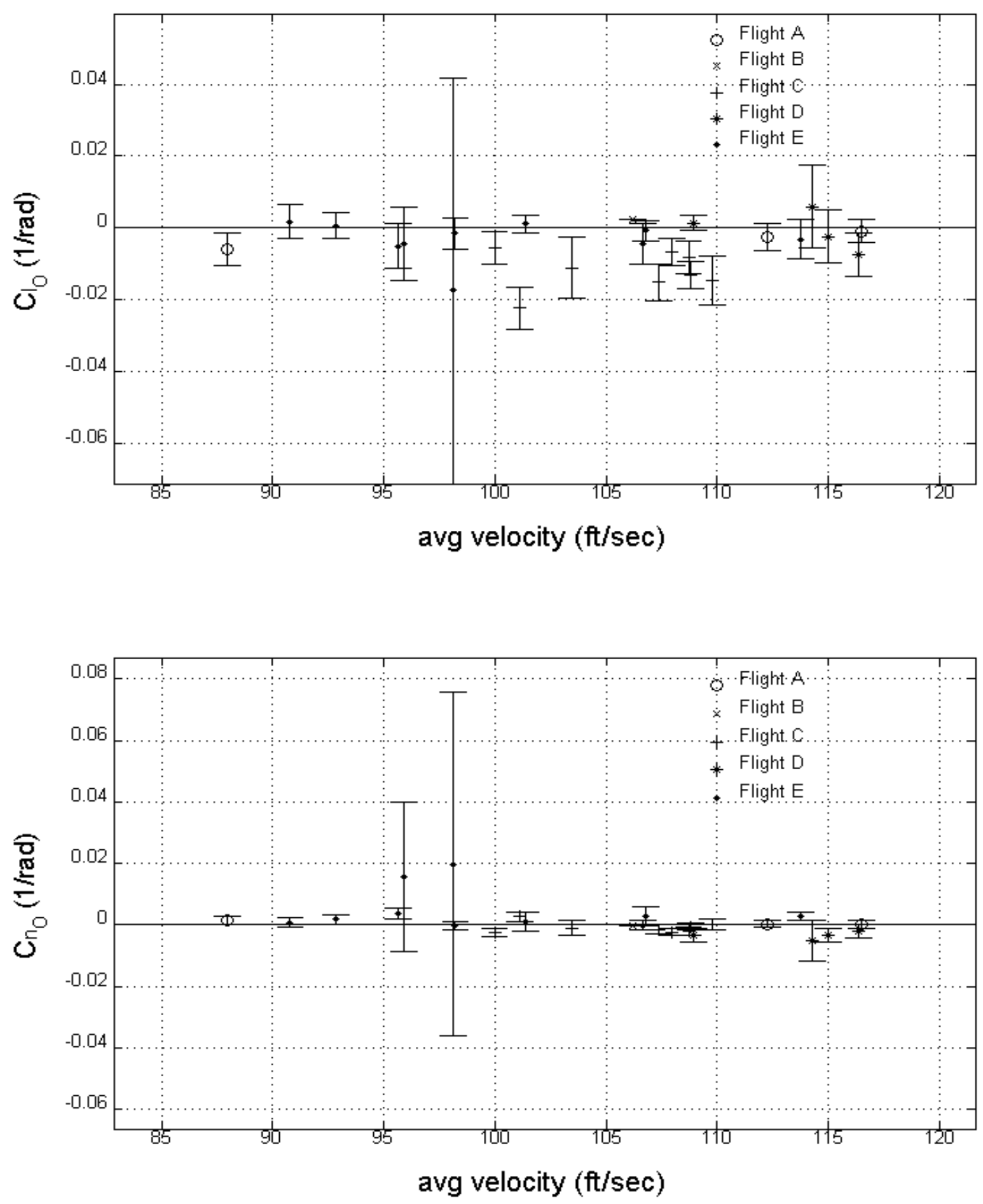

Figure 6.4.26 Off-line estimation results of $\mathrm{C}_{\mathrm{l}_{\mathrm{O}}}$ and $\mathrm{C}_{\mathrm{n}_{\mathrm{O}}}$ for lateral-directional Flights A, B, C, D, and E versus velocity 

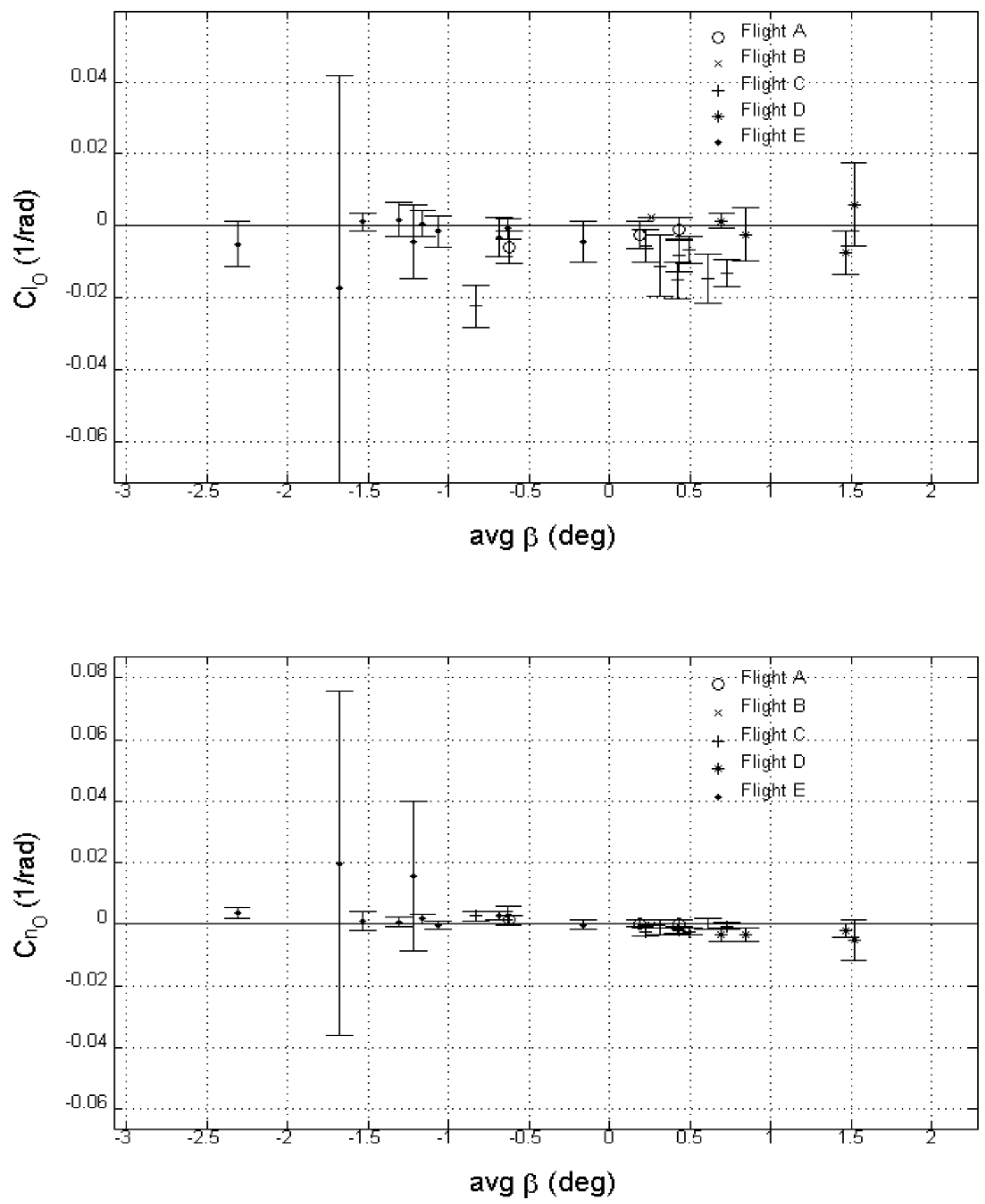

Figure 6.4.27 Off-line estimation results of $\mathrm{C}_{\mathrm{l}_{\mathrm{O}}}$ and $\mathrm{C}_{\mathrm{n}_{\mathrm{O}}}$ for lateral-directional Flights A, B, C, D, and E versus sideslip angle 

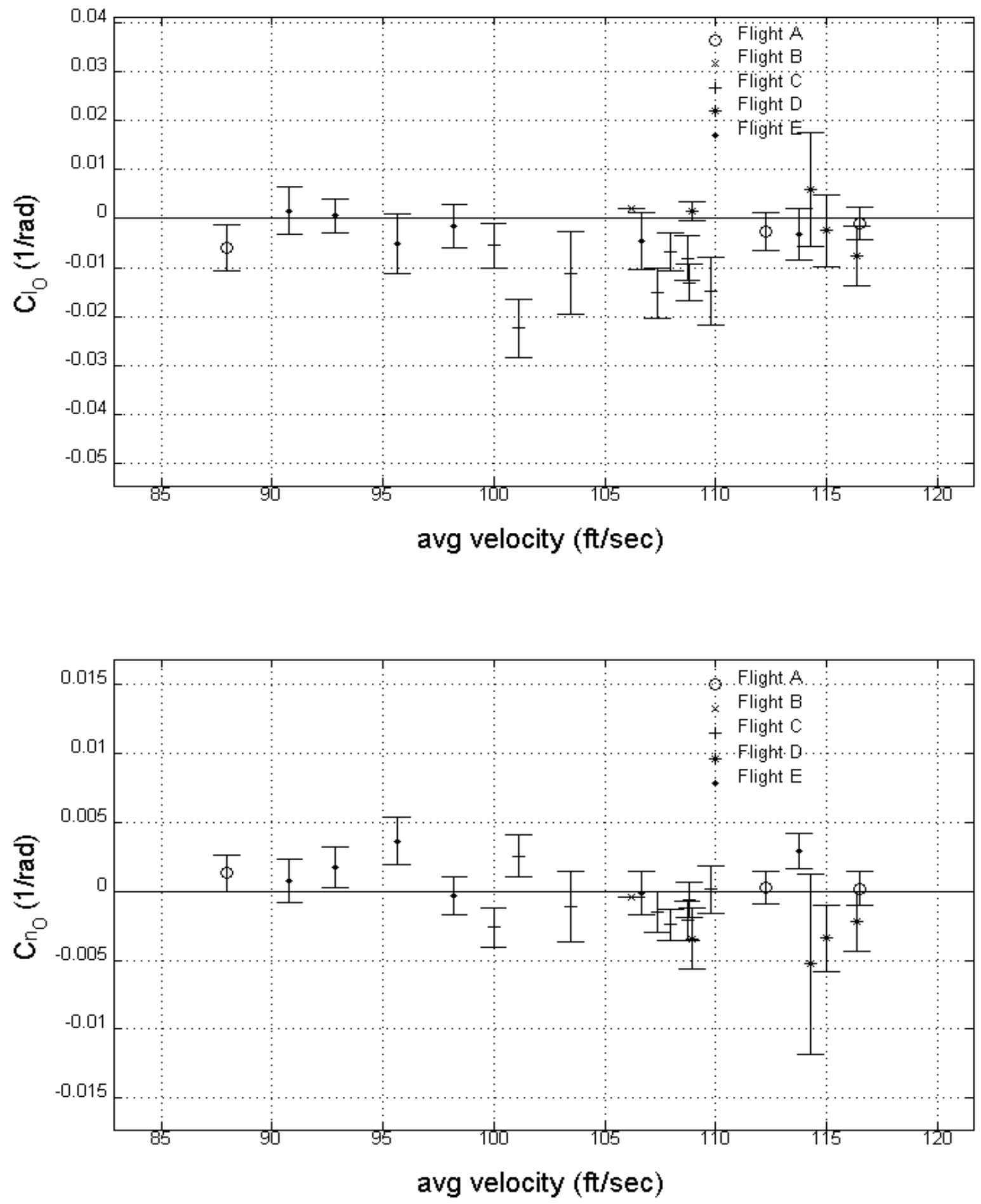

Figure 6.4.28 Off-line estimation results of $\mathrm{C}_{\mathrm{l}_{\mathrm{O}}}$ and $\mathrm{C}_{\mathrm{n}_{\mathrm{O}}}$ for lateral-directional Flights $\mathrm{A}, \mathrm{B}, \mathrm{C}, \mathrm{D}$, and $\mathrm{E}$ versus velocity (minus selected $\delta_{\mathrm{r}}$ inputs) 

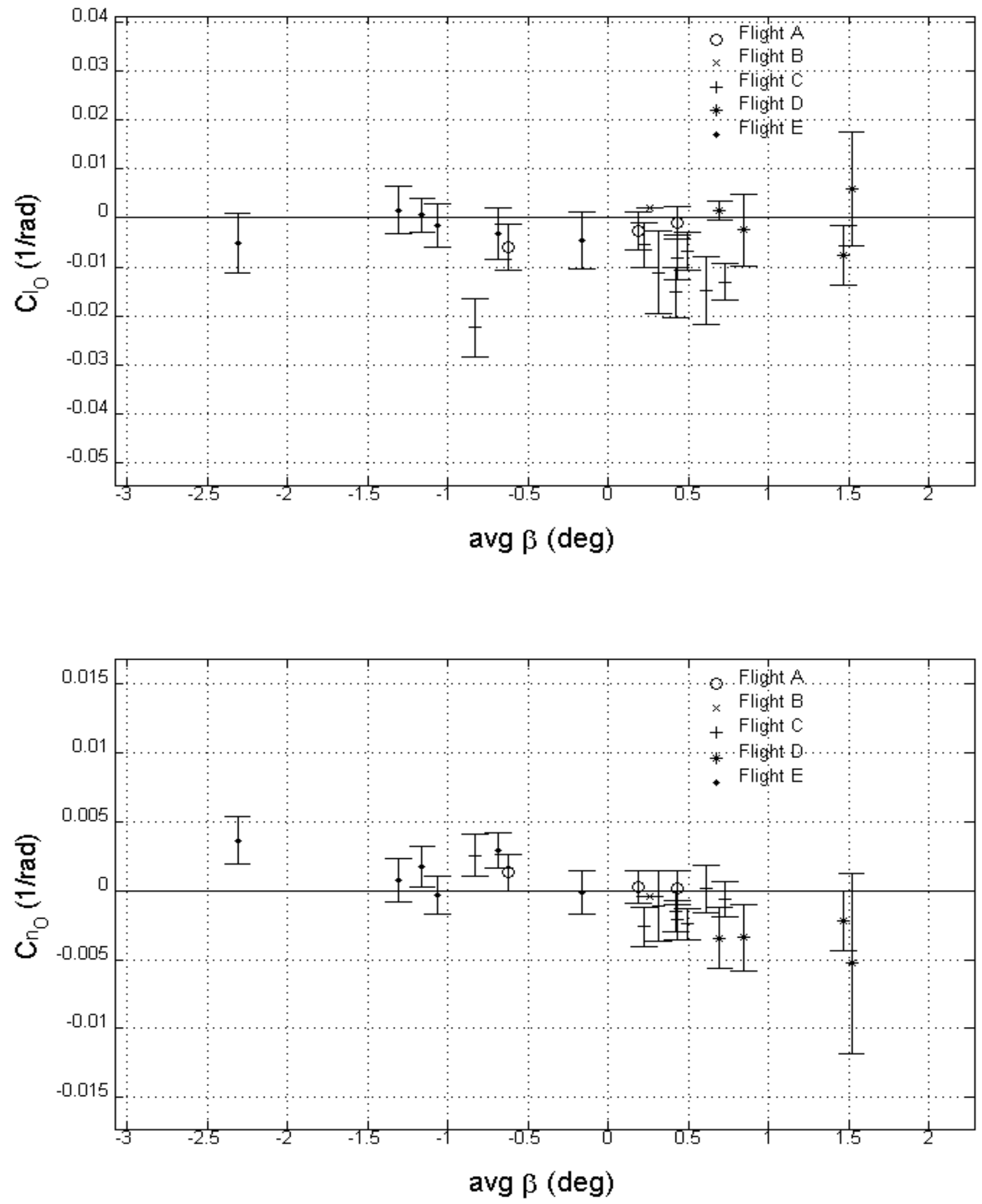

Figure 6.4.29 Off-line estimation results of $\mathrm{C}_{\mathrm{l}_{\mathrm{O}}}$ and $\mathrm{C}_{\mathrm{n}_{\mathrm{O}}}$ for lateral-directional Flights $\mathrm{A}, \mathrm{B}, \mathrm{C}, \mathrm{D}$, and $\mathrm{E}$ versus sideslip angle (minus selected $\delta_{\mathrm{r}}$ inputs) 

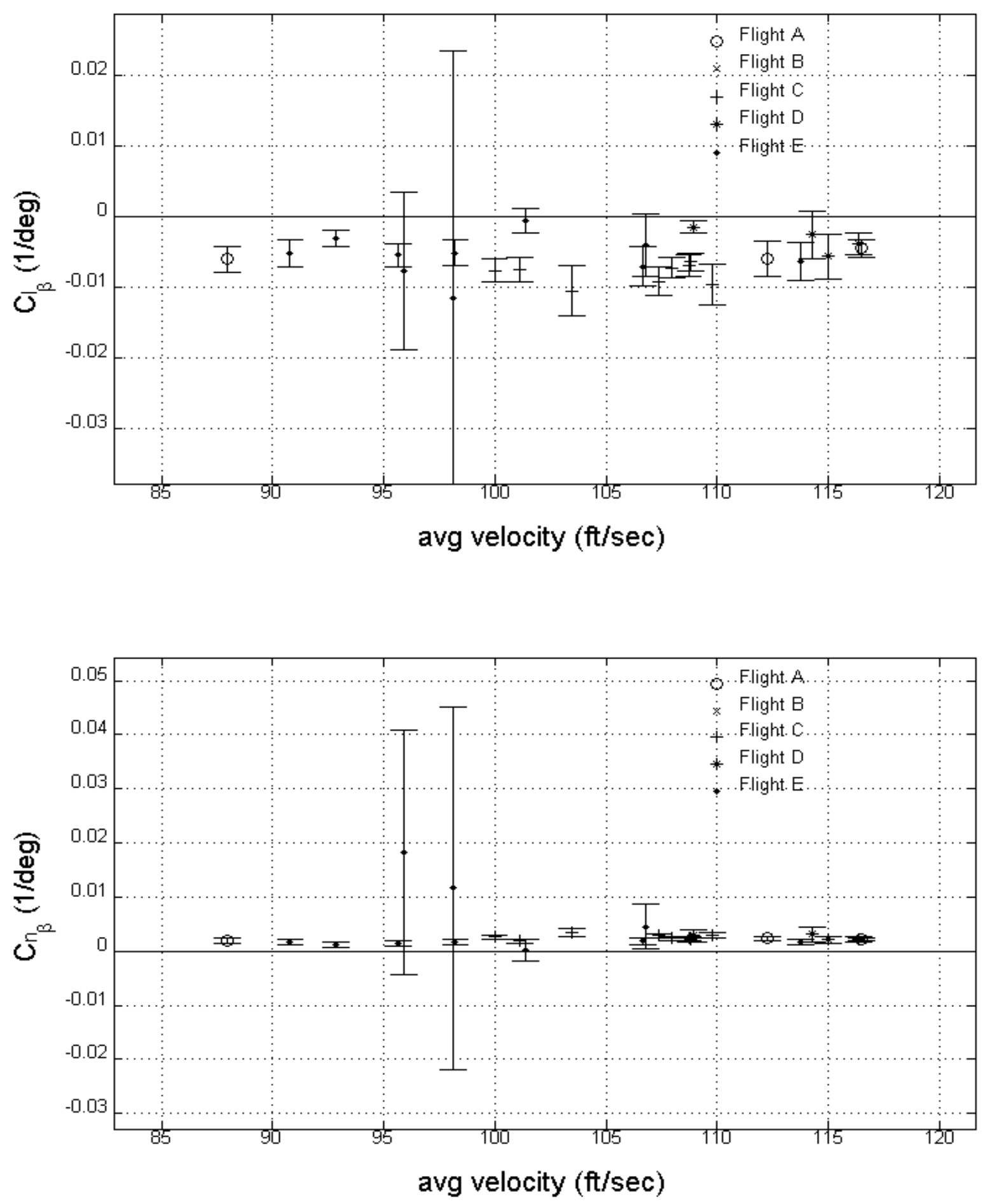

Figure 6.4.30 Off-line estimation results of $\mathrm{C}_{\mathrm{l}_{\beta}}$ and $\mathrm{C}_{\mathrm{n} \beta}$ for lateral-directional Flights A, B, C, D, and E versus velocity 

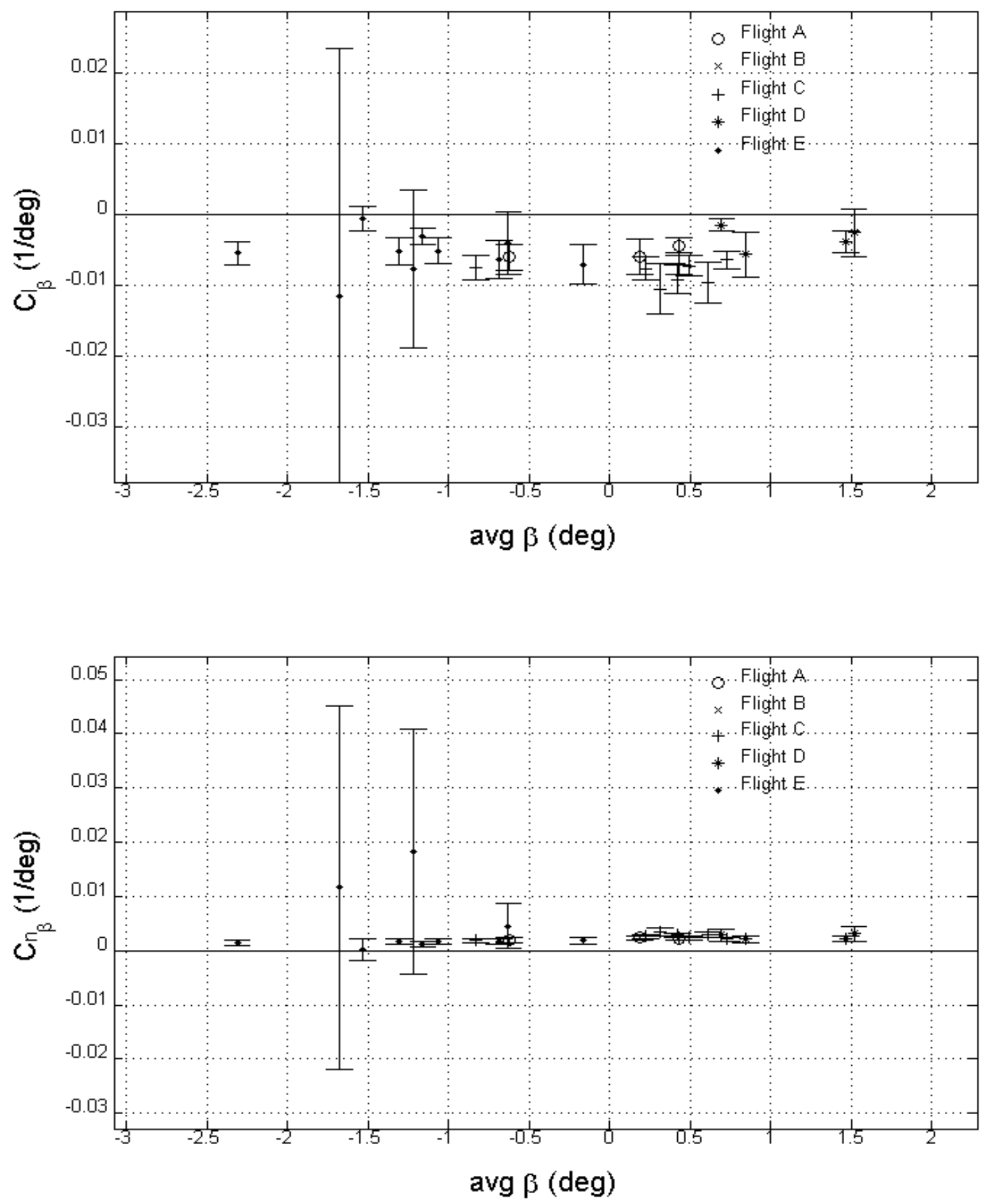

Figure 6.4.31 Off-line estimation results of $\mathrm{C}_{\mathrm{l}_{\beta}}$ and $\mathrm{C}_{\mathrm{n} \beta}$ for lateral-directional Flights A, B, C, D, and E versus sideslip angle 

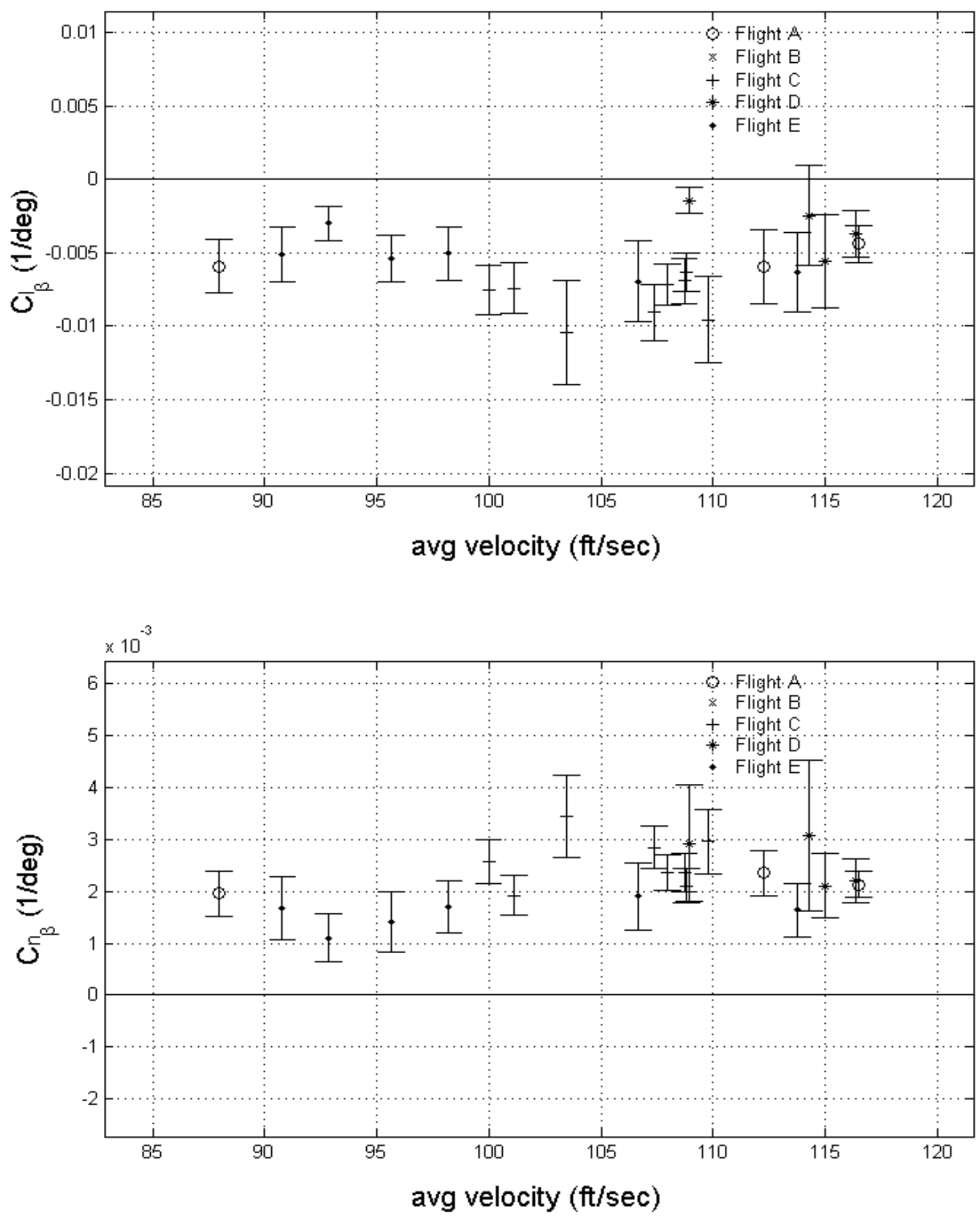

Figure 6.4.32 Off-line estimation results of $\mathrm{C}_{\mathrm{l}_{\beta}}$ and $\mathrm{C}_{\mathrm{n} \beta}$ for lateral-directional Flights $\mathrm{A}, \mathrm{B}, \mathrm{C}, \mathrm{D}$, and $\mathrm{E}$ versus velocity (minus selected $\delta_{\mathrm{r}}$ inputs) 

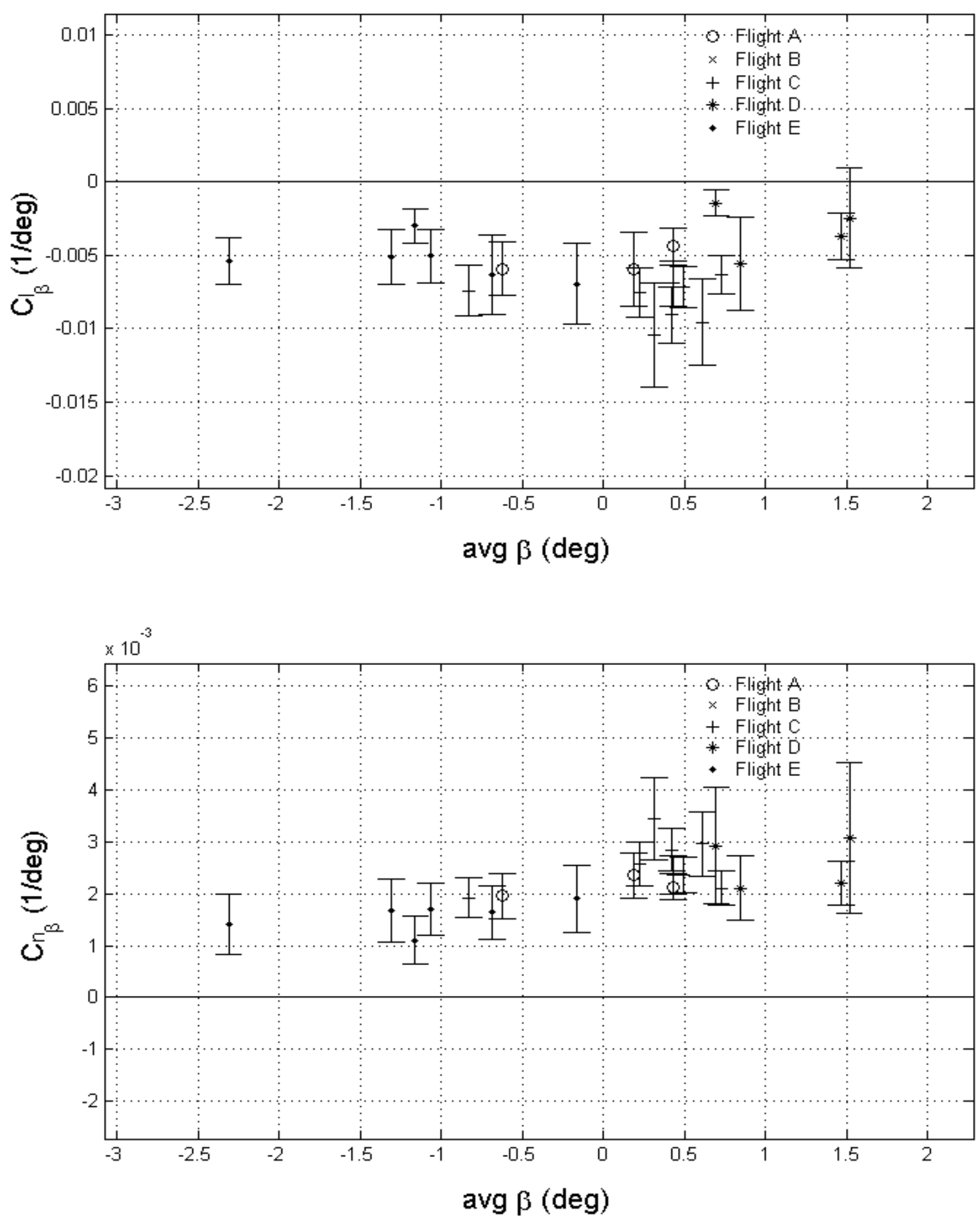

Figure 6.4.33 Off-line estimation results of $\mathrm{C}_{\mathrm{l}_{\beta}}$ and $\mathrm{C}_{\mathrm{n} \beta}$ for lateral-directional Flights A, B, C, D, and E versus sideslip angle (minus selected $\delta_{\mathrm{r}}$ inputs) 

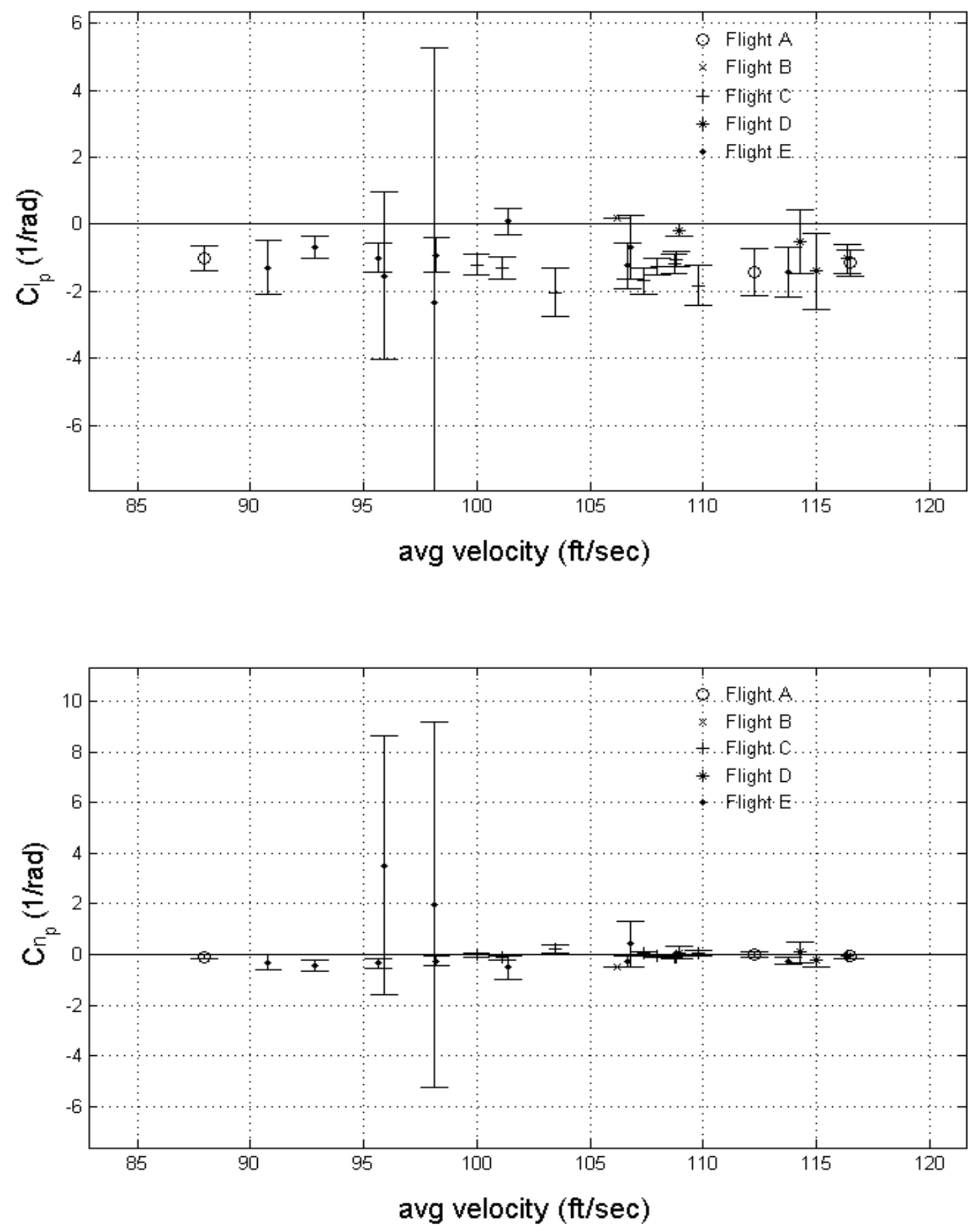

Figure 6.4.34 Off-line estimation results of $\mathrm{C}_{\mathrm{l}_{\mathrm{p}}}$ and $\mathrm{C}_{\mathrm{n}_{\mathrm{p}}}$ for lateral-directional Flights A, B, C, D, and E versus velocity 

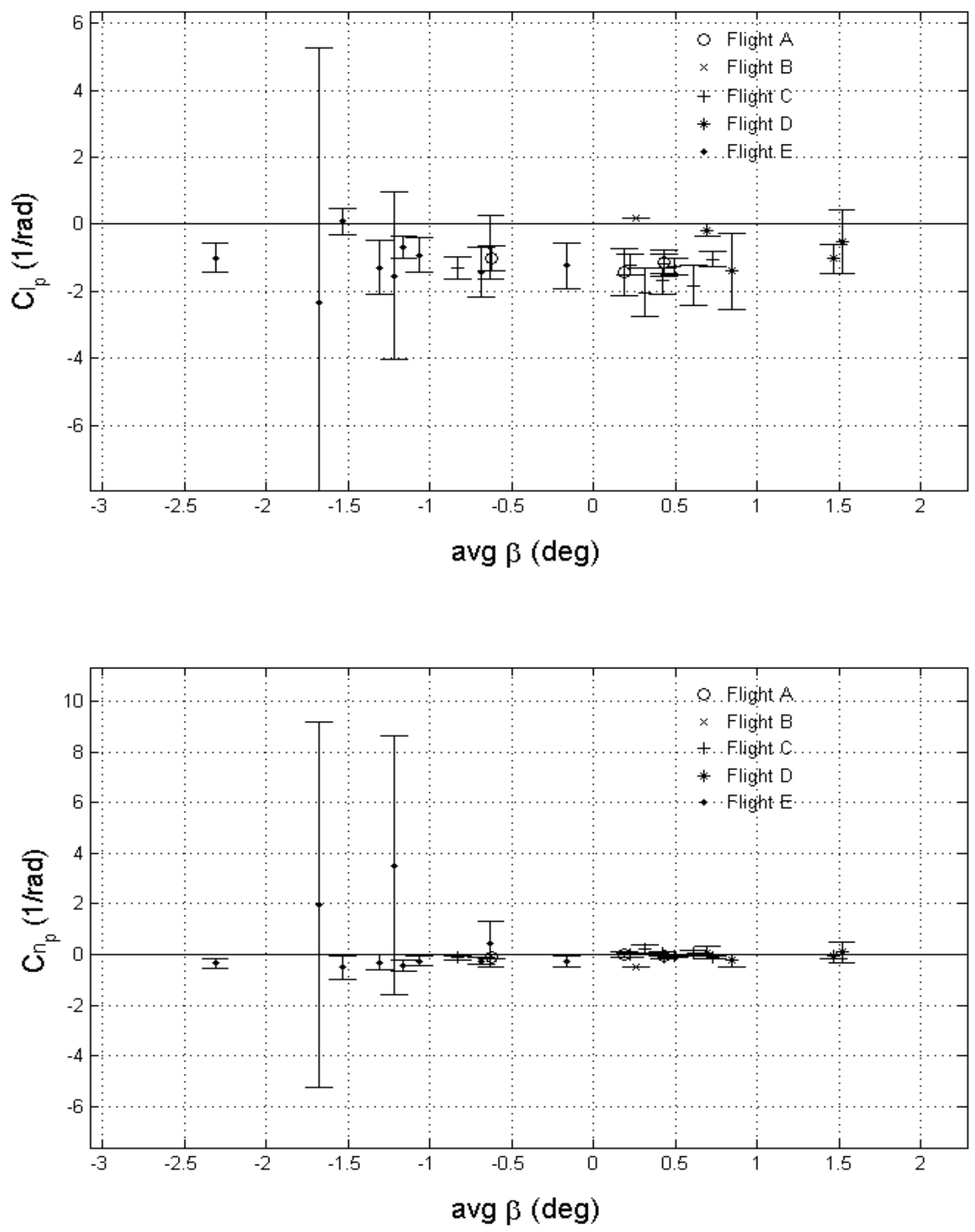

Figure 6.4.35 Off-line estimation results of $\mathrm{C}_{\mathrm{l}_{\mathrm{p}}}$ and $\mathrm{C}_{\mathrm{n}_{\mathrm{p}}}$ for lateral-directional Flights A, B, C, D, and E versus sideslip angle 

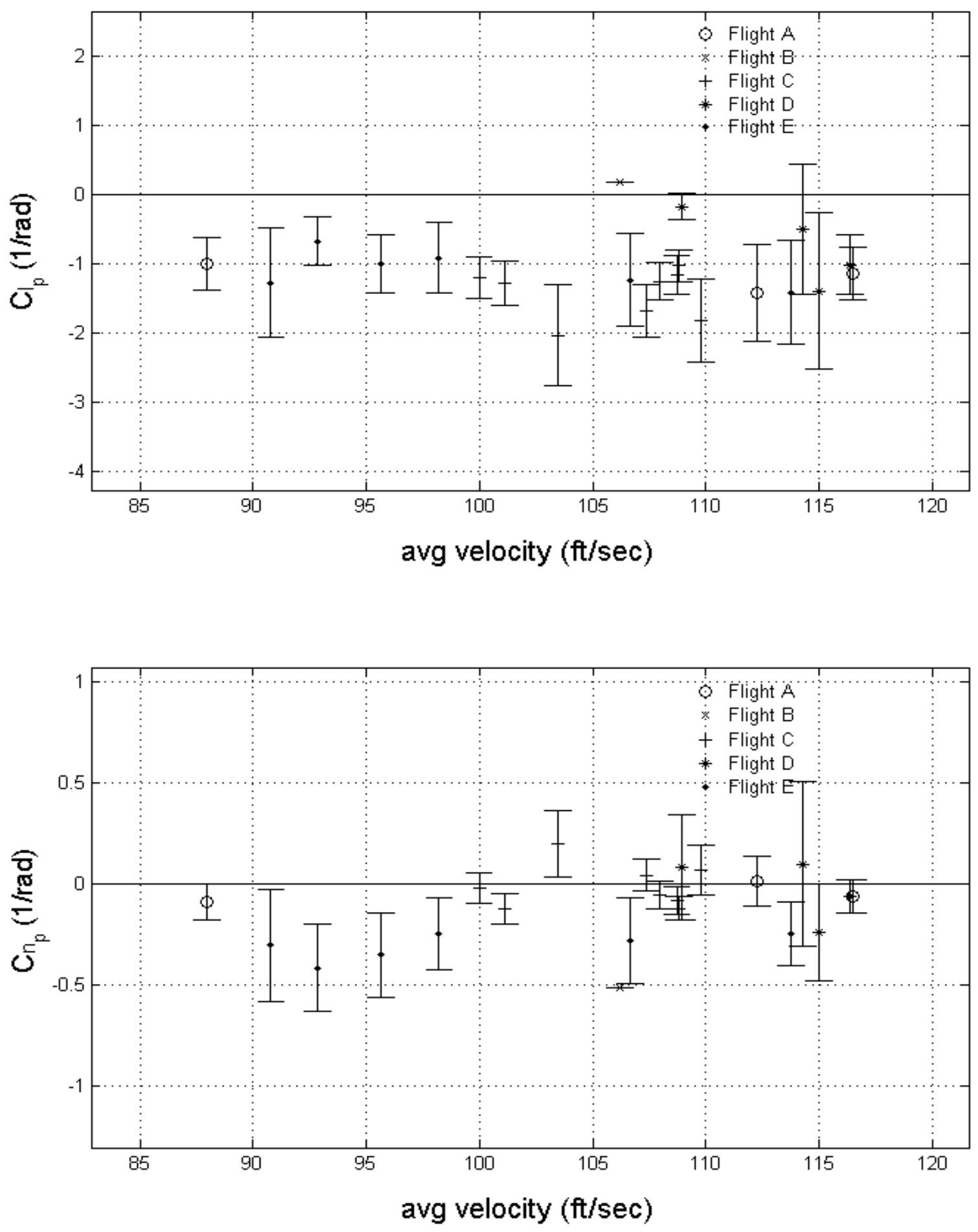

Figure 6.4.36 Off-line estimation results of $\mathrm{C}_{\mathrm{l}_{\mathrm{p}}}$ and $\mathrm{C}_{\mathrm{n}_{\mathrm{p}}}$ for lateral-directional Flights A, B, C, D, and E versus velocity (minus selected $\delta_{\mathrm{r}}$ inputs) 

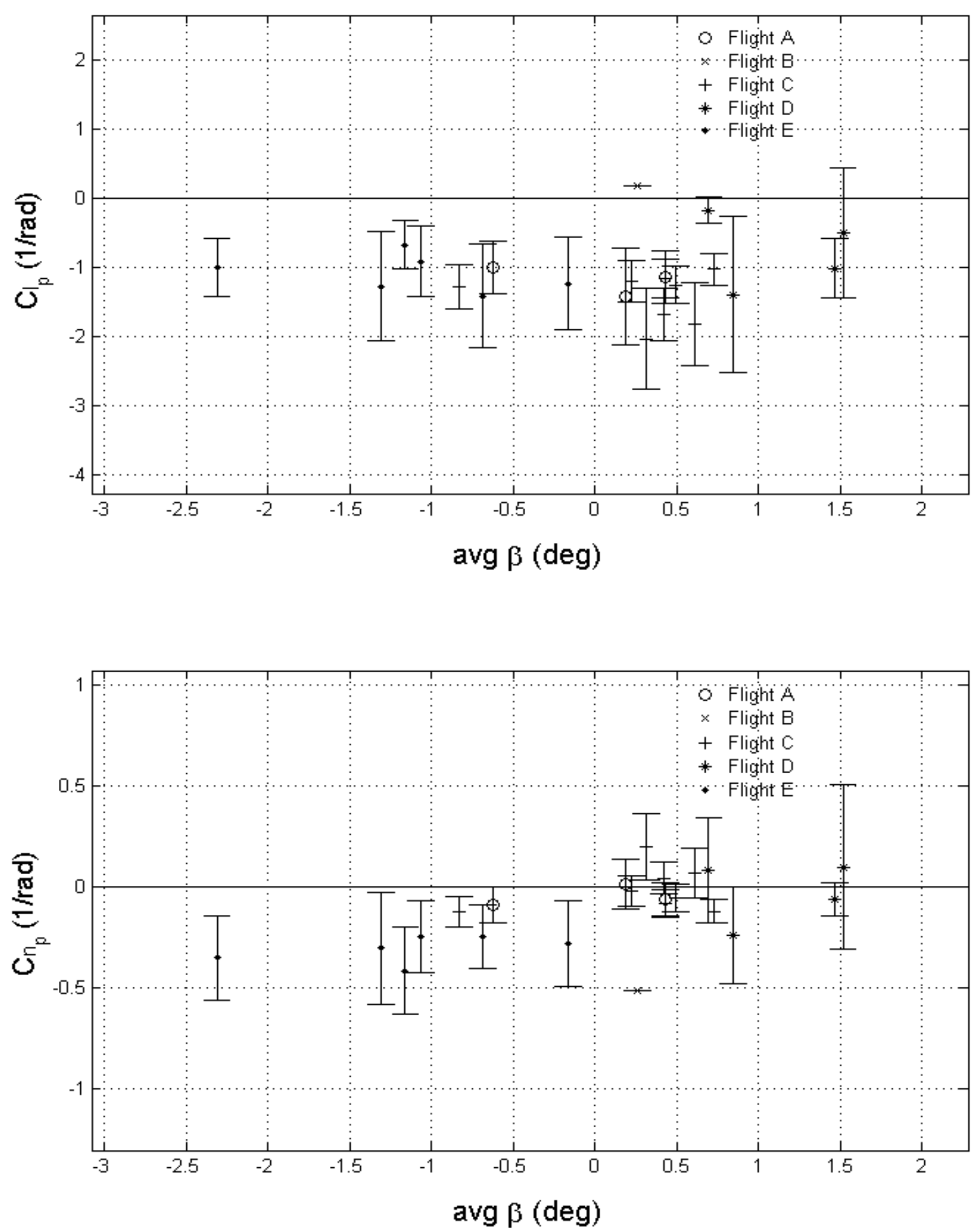

Figure 6.4.37 Off-line estimation results of $\mathrm{C}_{\mathrm{l}_{\mathrm{p}}}$ and $\mathrm{C}_{\mathrm{n}_{\mathrm{p}}}$ for lateral-directional Flights A, B, C, D, and E versus sideslip angle (minus selected $\delta_{\mathrm{r}}$ inputs) 

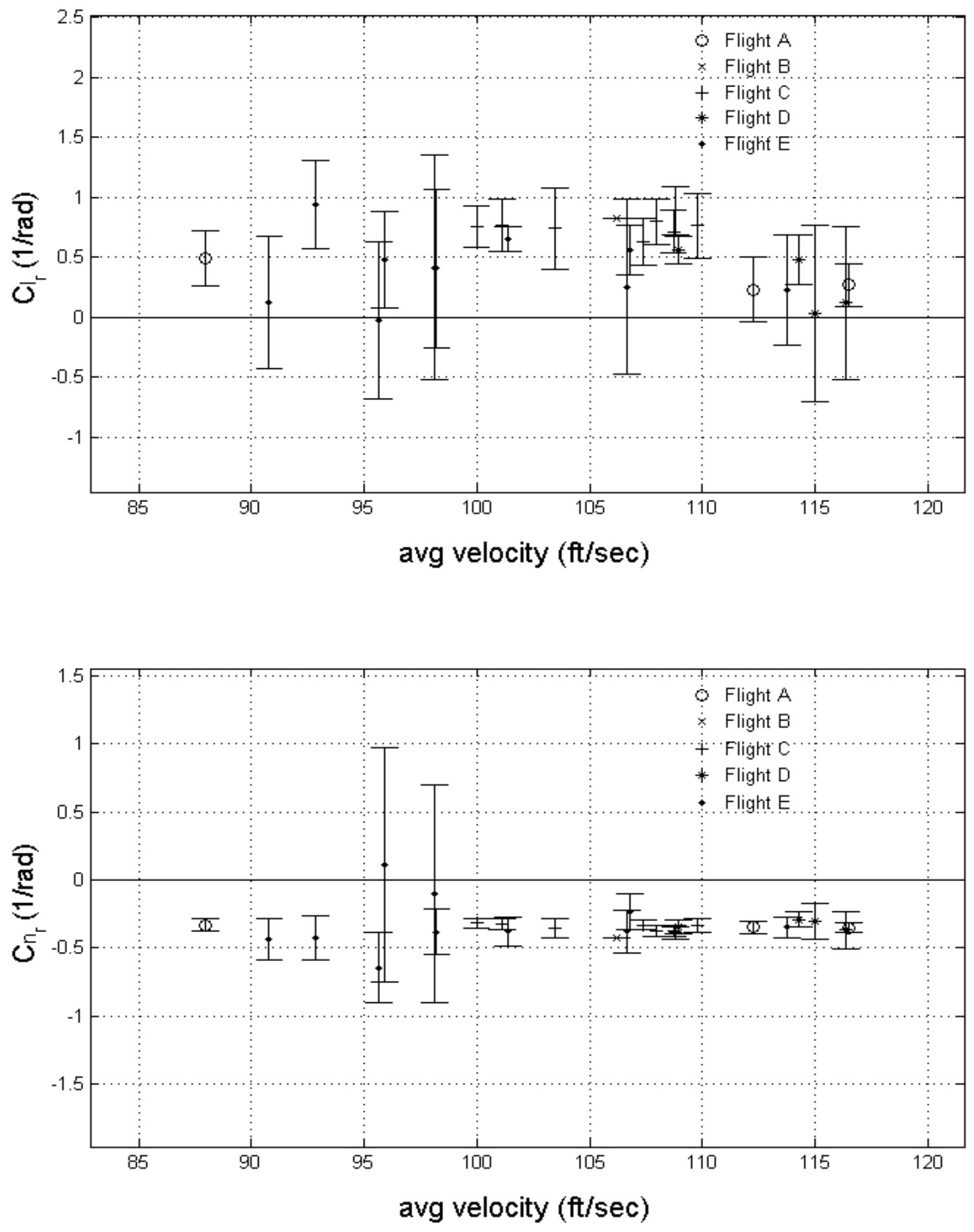

Figure 6.4.38 Off-line estimation results of $\mathrm{C}_{\mathrm{l}_{\mathrm{r}}}$ and $\mathrm{C}_{\mathrm{n}_{\mathrm{r}}}$ for lateral-directional Flights A, B, C, D, and $\mathrm{E}$ versus velocity 

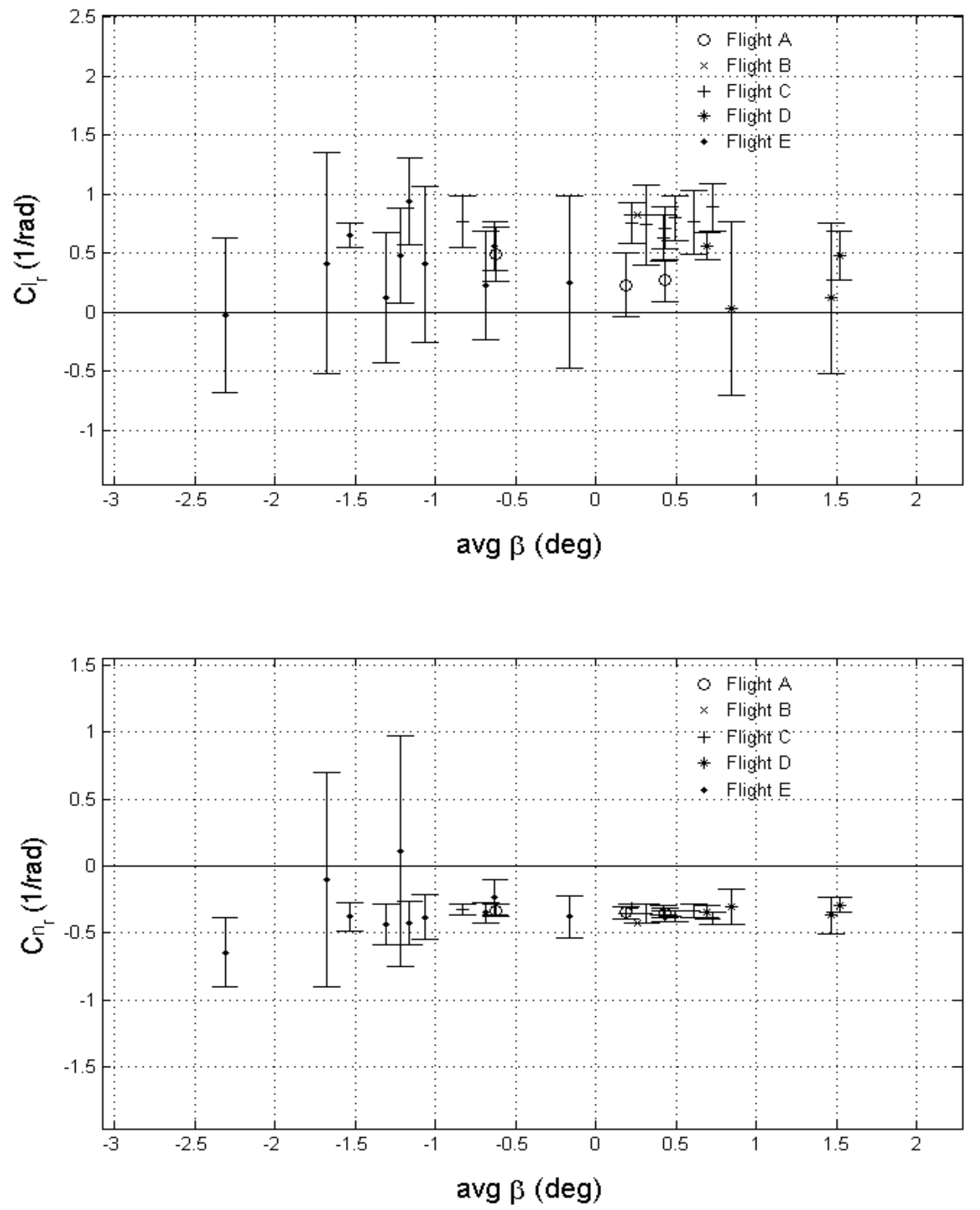

Figure 6.4.39 Off-line estimation results of $\mathrm{C}_{\mathrm{l}_{\mathrm{r}}}$ and $\mathrm{C}_{\mathrm{n}_{\mathrm{r}}}$ for lateral-directional Flights A, B, C, D, and E versus sideslip angle 

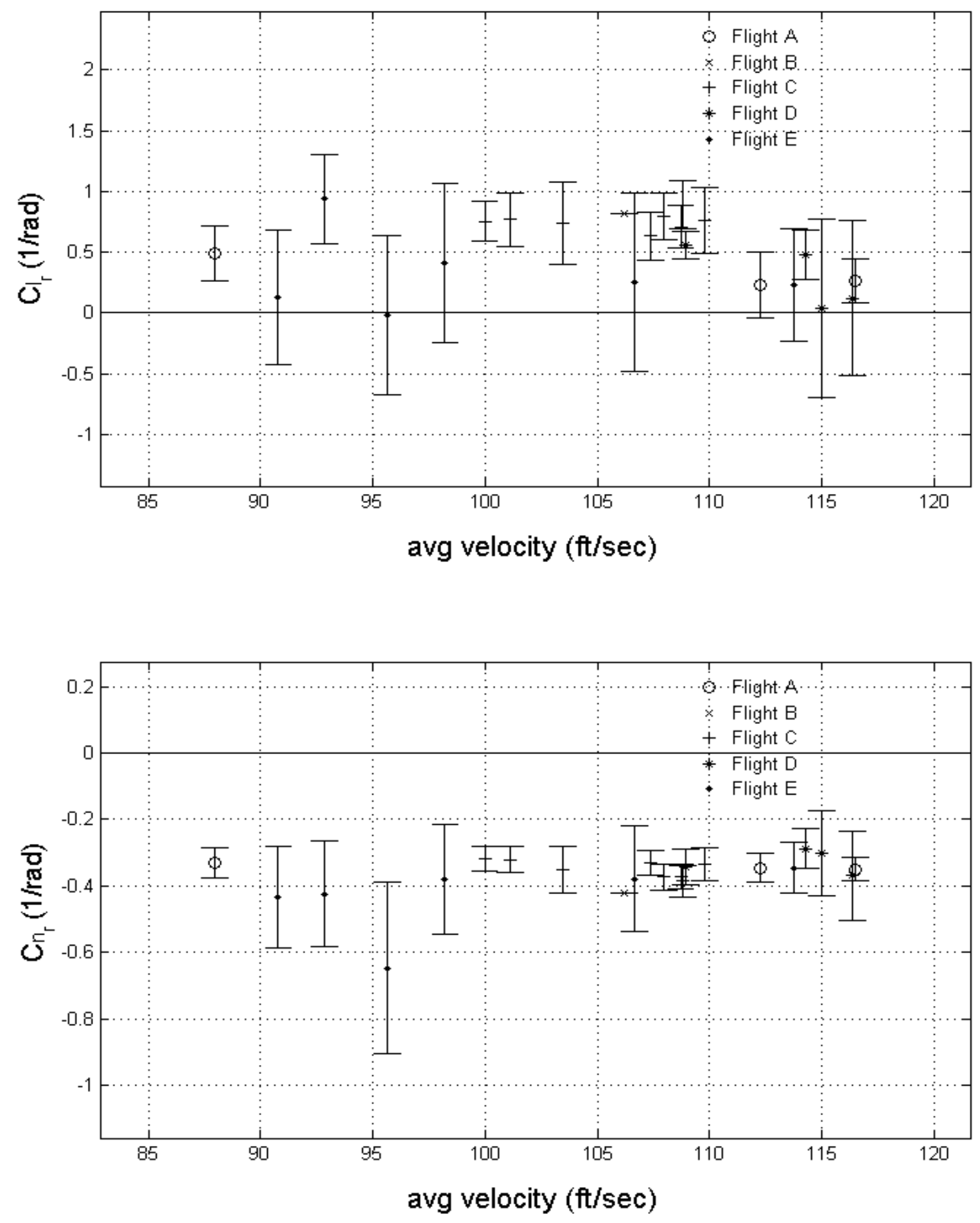

Figure 6.4.40 Off-line estimation results of $\mathrm{C}_{\mathrm{l}_{\mathrm{r}}}$ and $\mathrm{C}_{\mathrm{n}_{\mathrm{r}}}$ for lateral-directional Flights $\mathrm{A}, \mathrm{B}, \mathrm{C}, \mathrm{D}$, and $\mathrm{E}$ versus velocity (minus selected $\delta_{\mathrm{r}}$ inputs) 

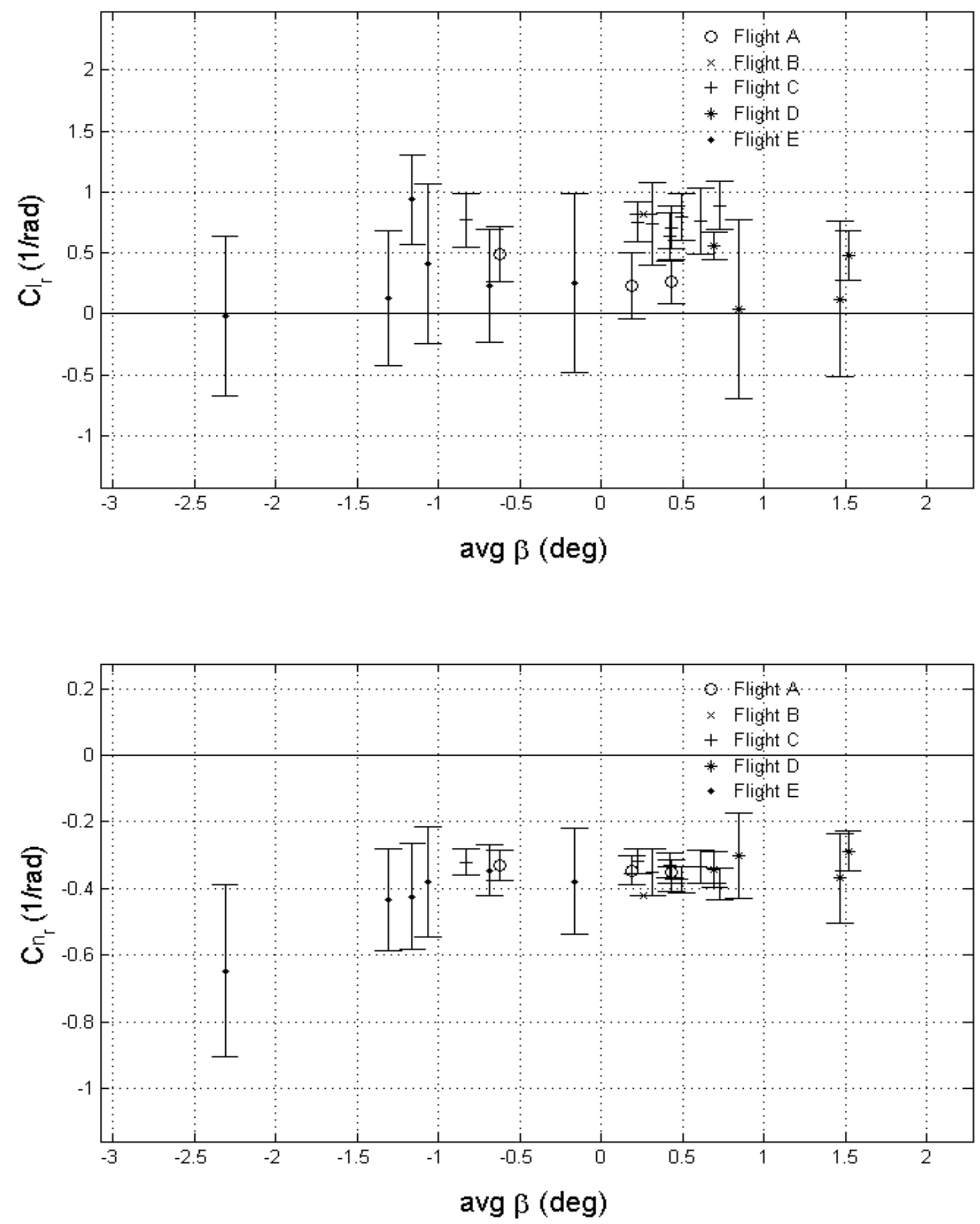

Figure 6.4.41 Off-line estimation results of $\mathrm{C}_{\mathrm{l}_{\mathrm{r}}}$ and $\mathrm{C}_{\mathrm{n}_{\mathrm{r}}}$ for lateral-directional Flights A, B, C, D, and E versus sideslip angle (minus selected $\delta_{\mathrm{r}}$ inputs) 

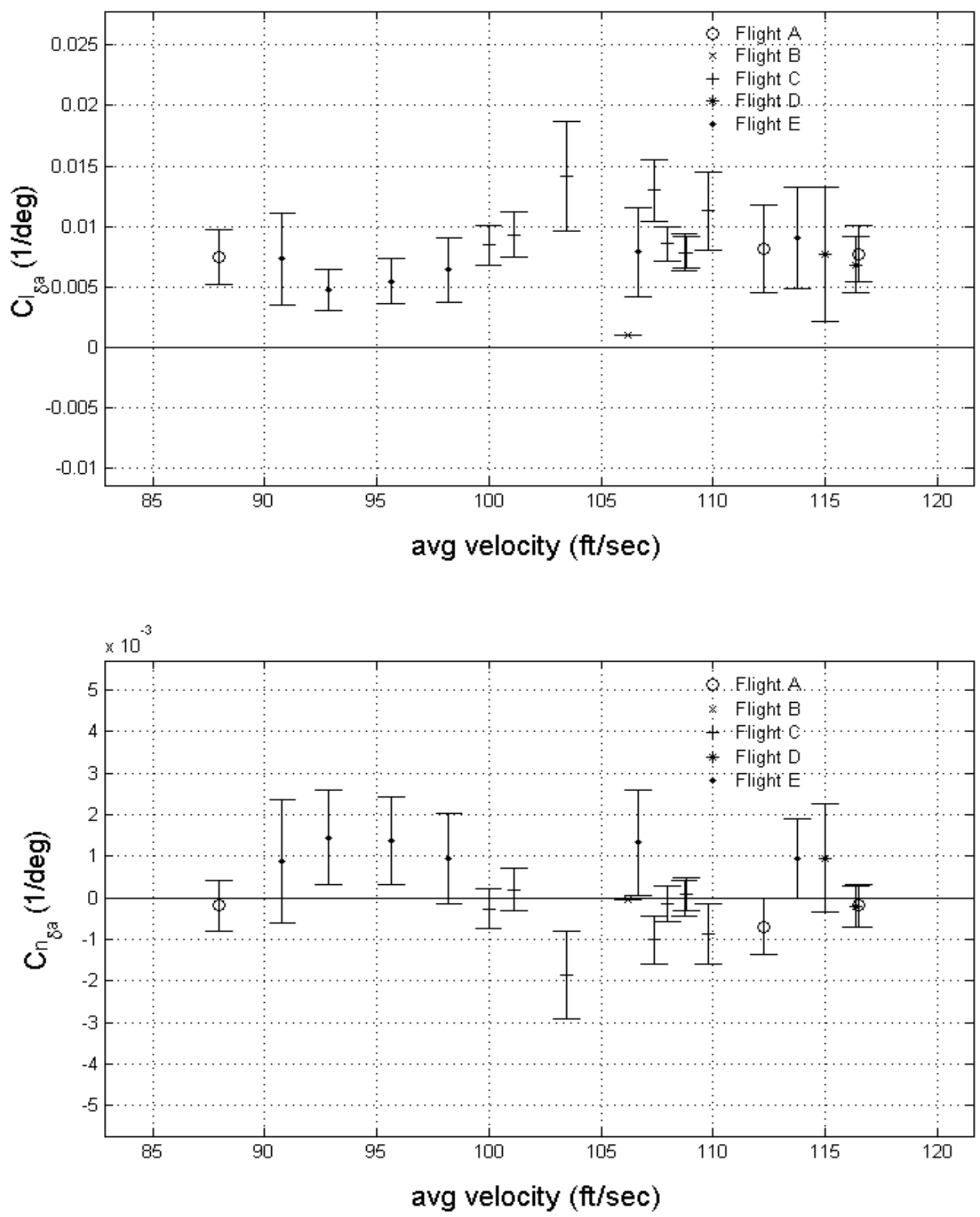

Figure 6.4.42 Off-line estimation results of $\mathrm{C}_{\delta_{\delta_{a}}}$ and $\mathrm{C}_{\mathrm{n}_{\mathrm{a}}}$ for lateral-directional Flights A, B, C, D, and E versus velocity 

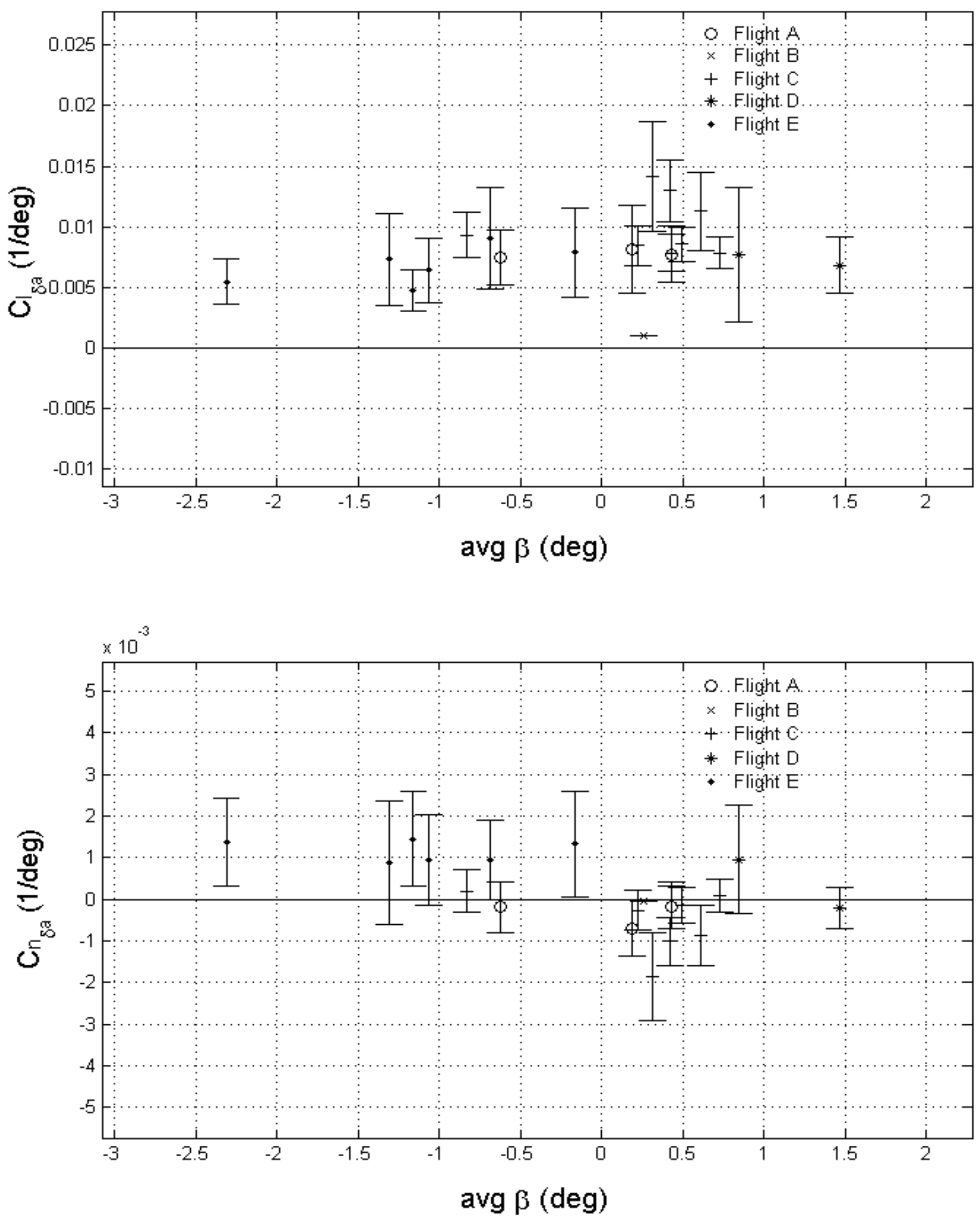

Figure 6.4.43 Off-line estimation results of $\mathrm{C}_{\mathrm{l}_{\mathrm{a}}}$ and $\mathrm{C}_{\mathrm{n}_{\delta_{\mathrm{a}}}}$ for lateral-directional Flights A, B, C, D, and E versus sideslip angle 

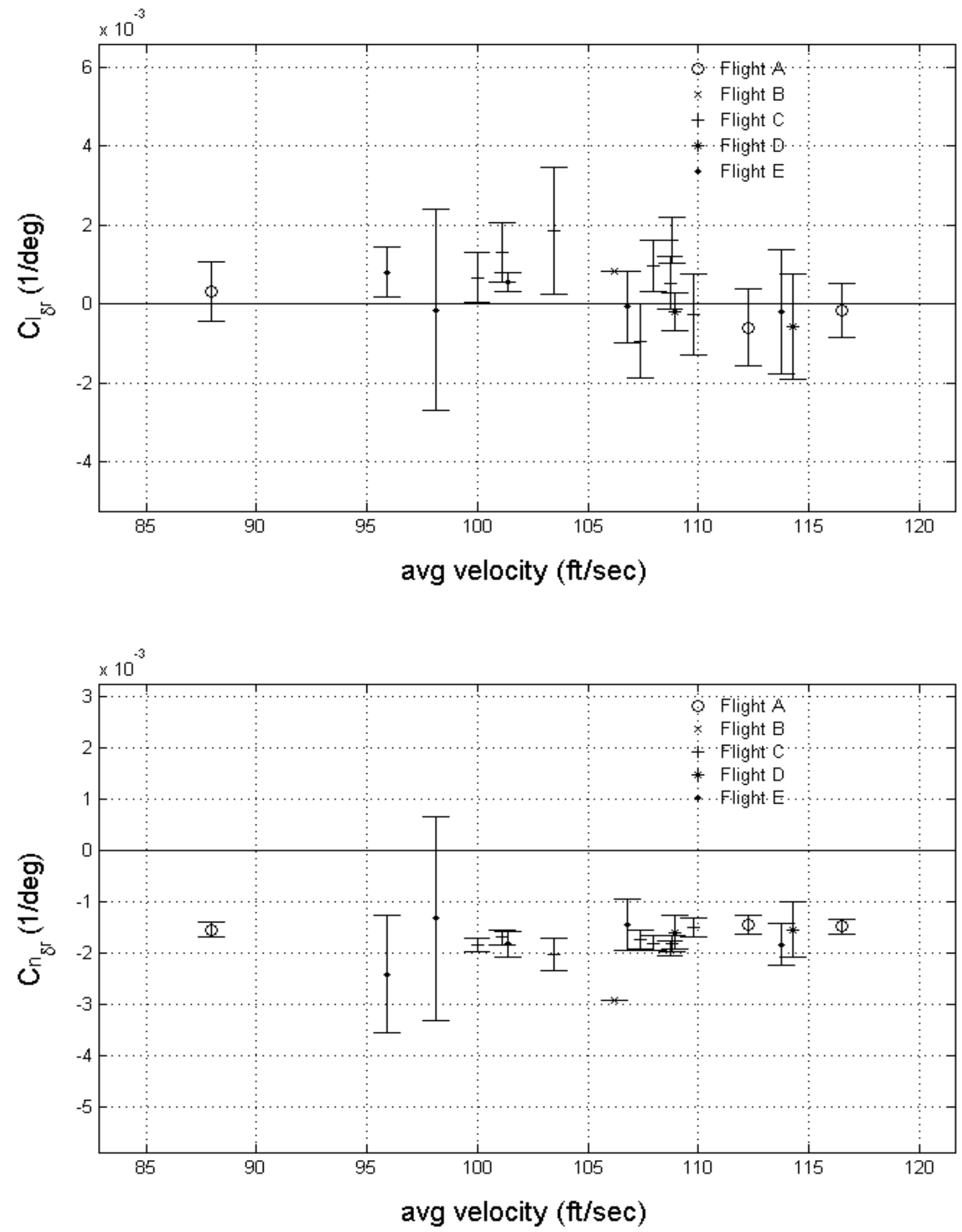

Figure 6.4.44 Off-line estimation results of $\mathrm{C}_{\mathrm{l}_{\mathrm{r}}}$ and $\mathrm{C}_{\mathrm{n}_{\delta_{\mathrm{r}}}}$ for lateral-directional Flights A, B, C, D, and E versus velocity 

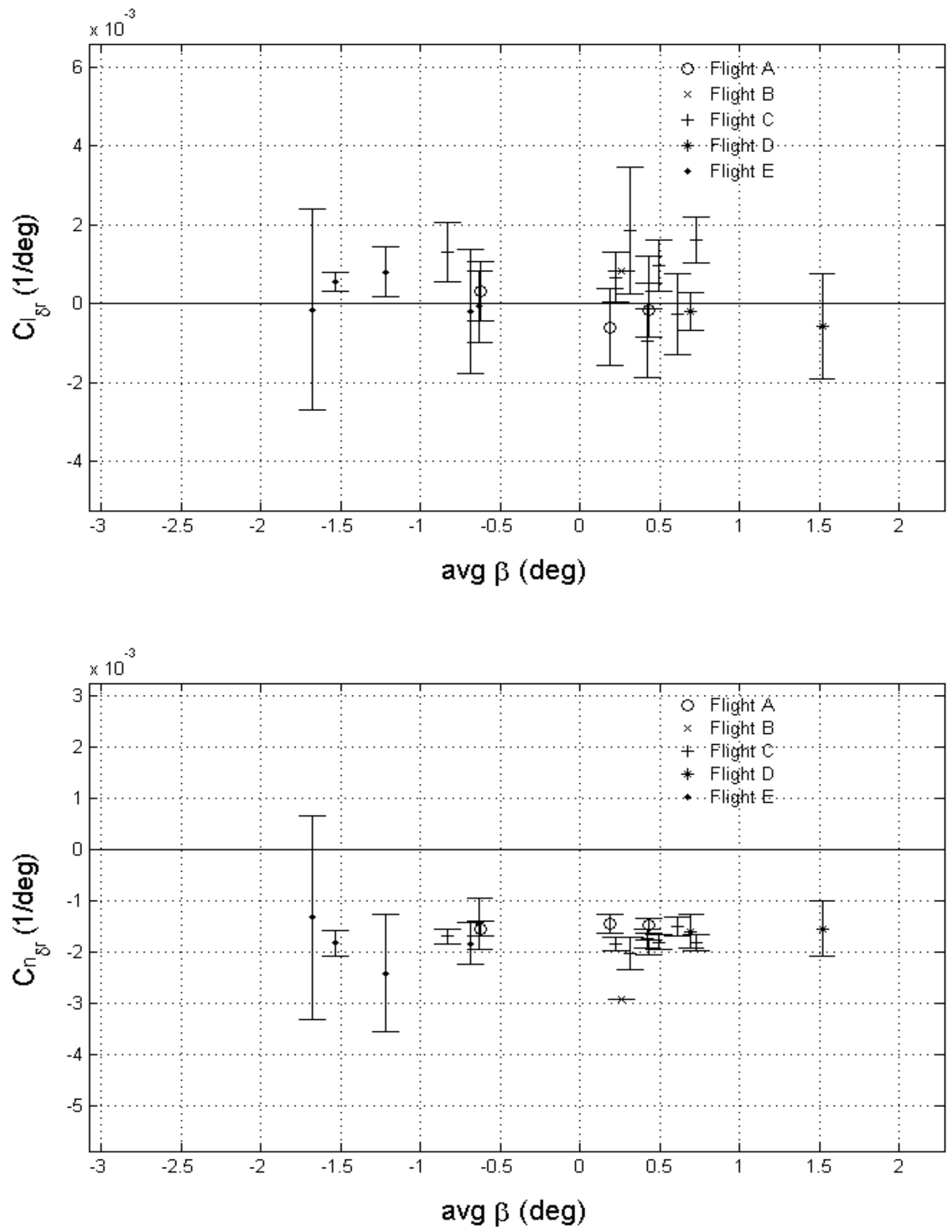

Figure 6.4.45 Off-line estimation results of $\mathrm{C}_{\delta_{\mathrm{r}}}$ and $\mathrm{C}_{\mathrm{n}_{\delta_{\mathrm{r}}}}$ for lateral-directional Flights A, B, C, D, and E versus sideslip angle 

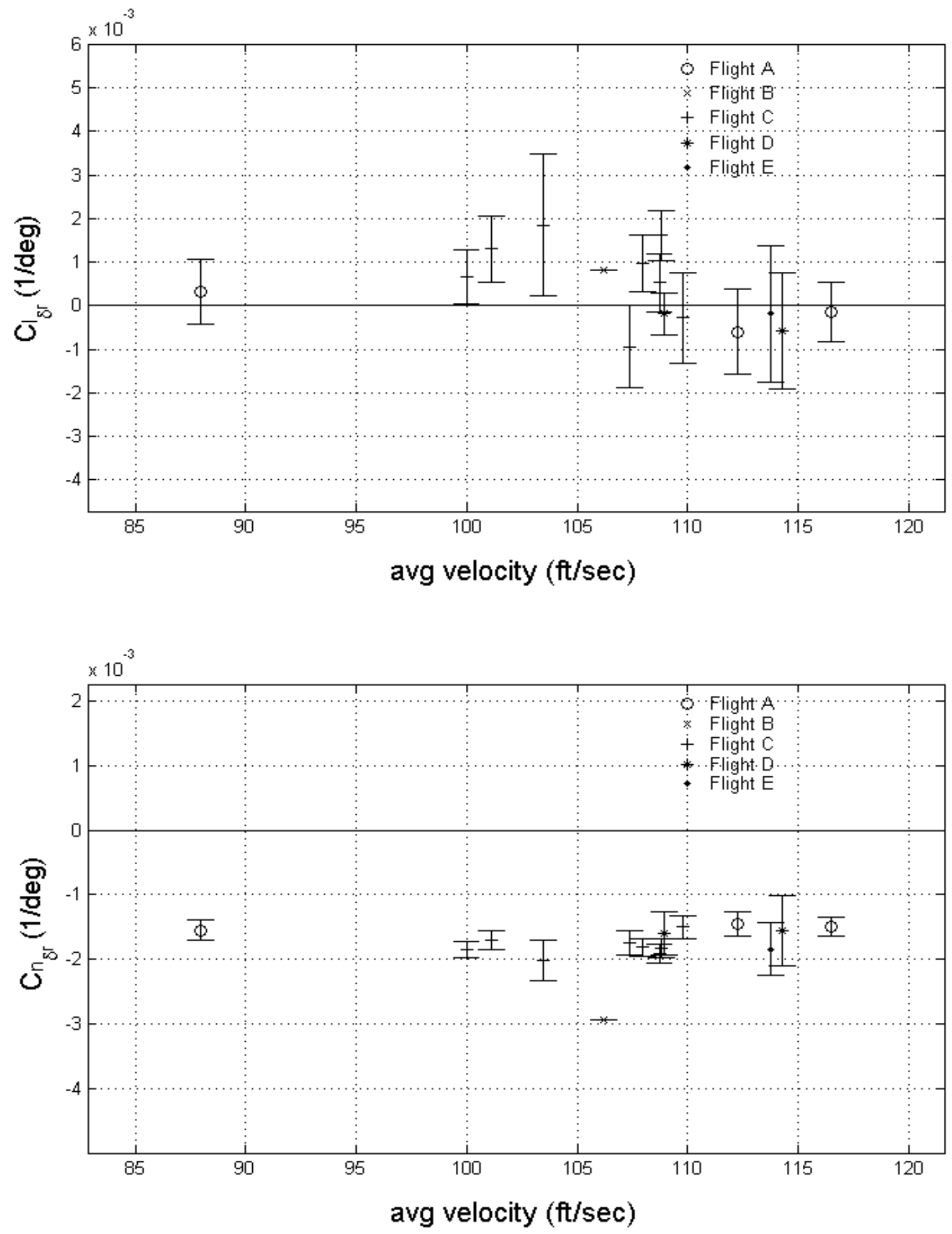

Figure 6.4.46 Off-line estimation results of $\mathrm{C}_{\mathrm{l}_{\mathrm{r}}}$ and $\mathrm{C}_{\mathrm{n}_{\delta_{\mathrm{r}}}}$ for lateral-directional Flights A, B, C, D, and E versus velocity (minus selected $\delta_{\mathrm{r}}$ inputs) 

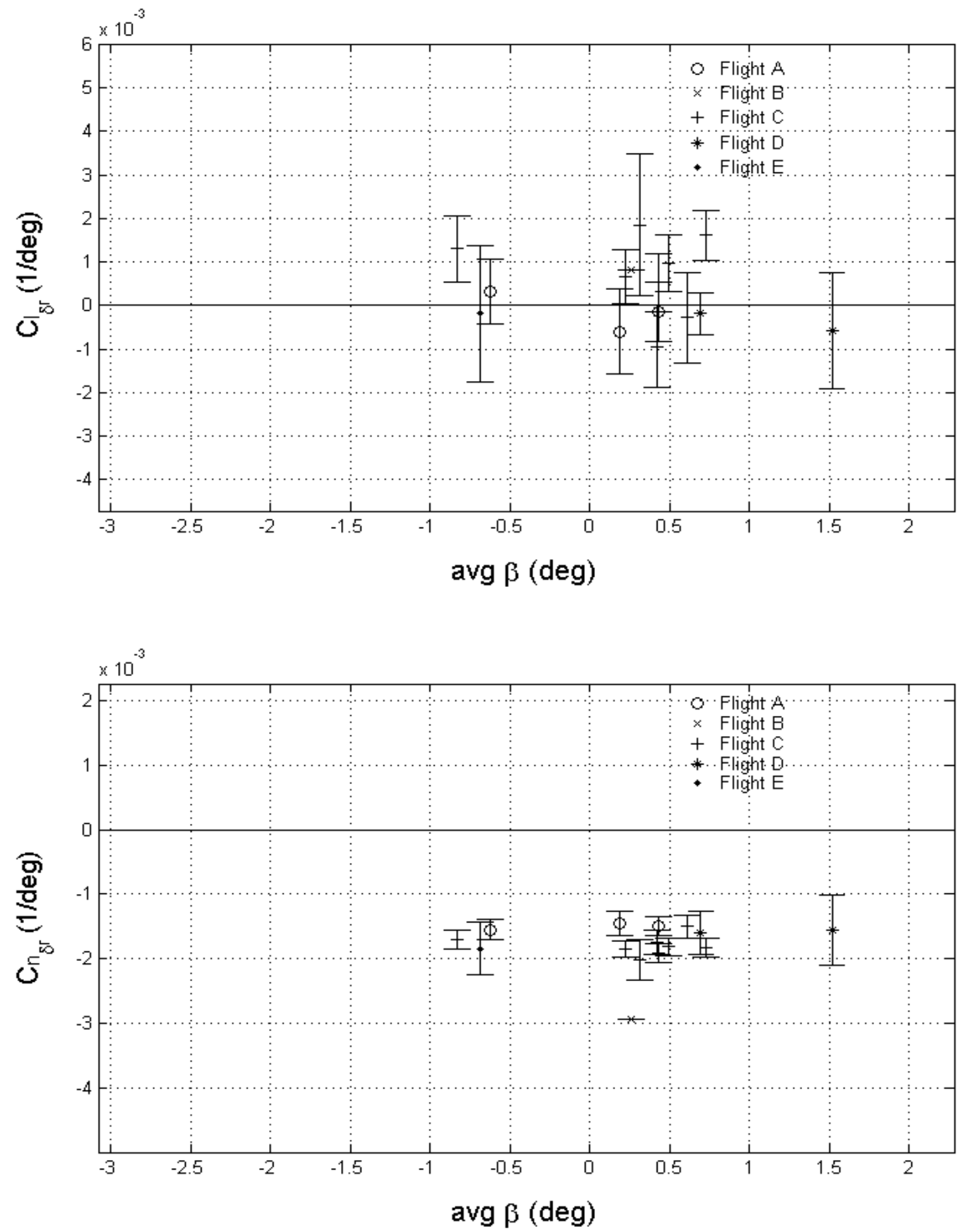

Figure 6.4.47 Off-line estimation results of $\mathrm{C}_{\delta_{\delta_{\mathrm{r}}}}$ and $\mathrm{C}_{\mathrm{n}_{\delta_{\mathrm{r}}}}$ for lateral-directional Flights A, B, C, D, and E versus sideslip angle (minus selected $\delta_{\mathrm{r}}$ inputs) 

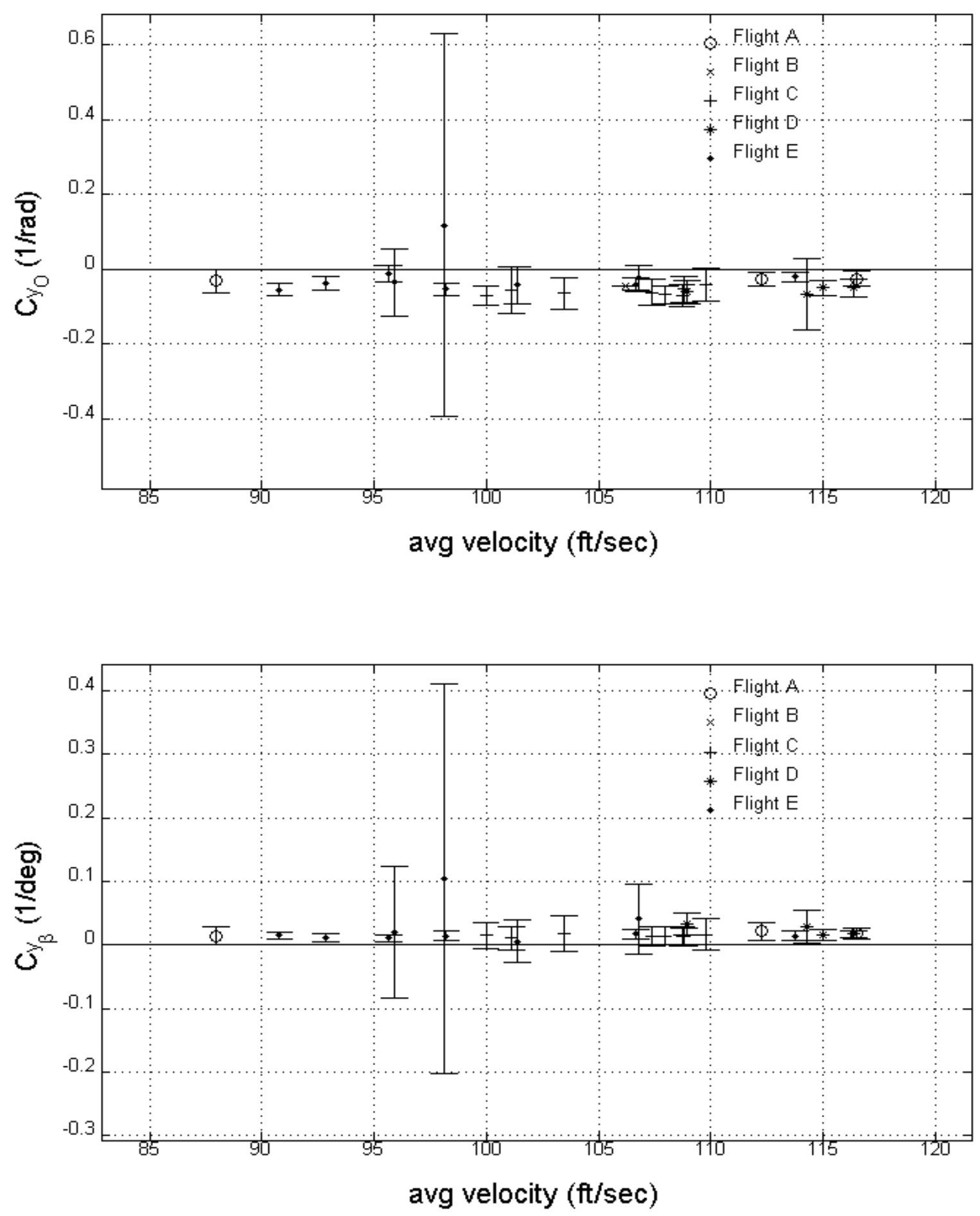

Figure 6.4.48 Off-line estimation results of $\mathrm{C}_{\mathrm{y}_{\mathrm{o}}}$ and $\mathrm{C}_{\mathrm{y} \beta}$ for lateral-directional Flights A, B, C, D, and E versus velocity 

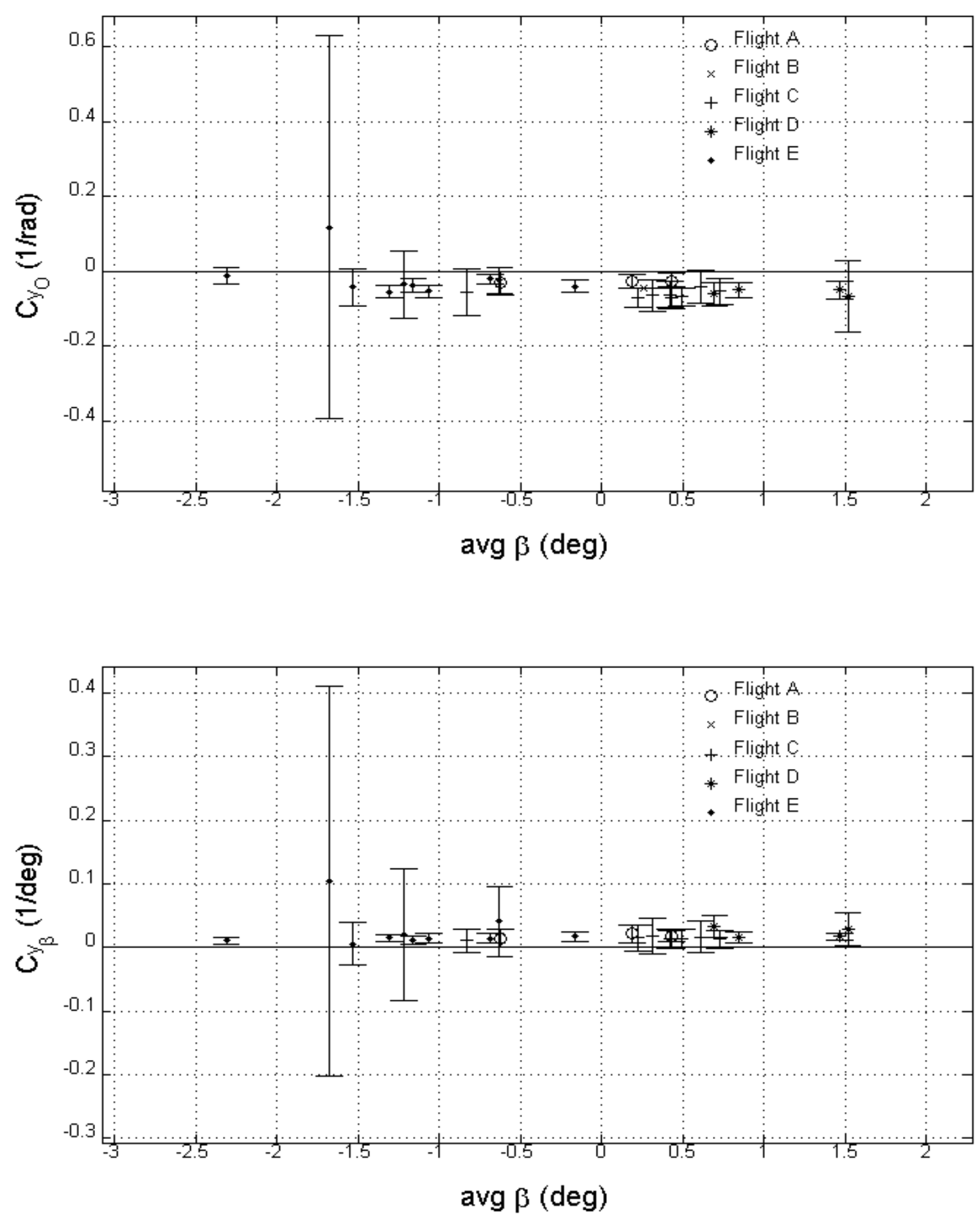

Figure 6.4.49 Off-line estimation results of $\mathrm{C}_{\mathrm{y}_{\mathrm{o}}}$ and $\mathrm{C}_{\mathrm{y} \beta}$ for lateral-directional Flights A, B, C, D, and E versus sideslip angle 

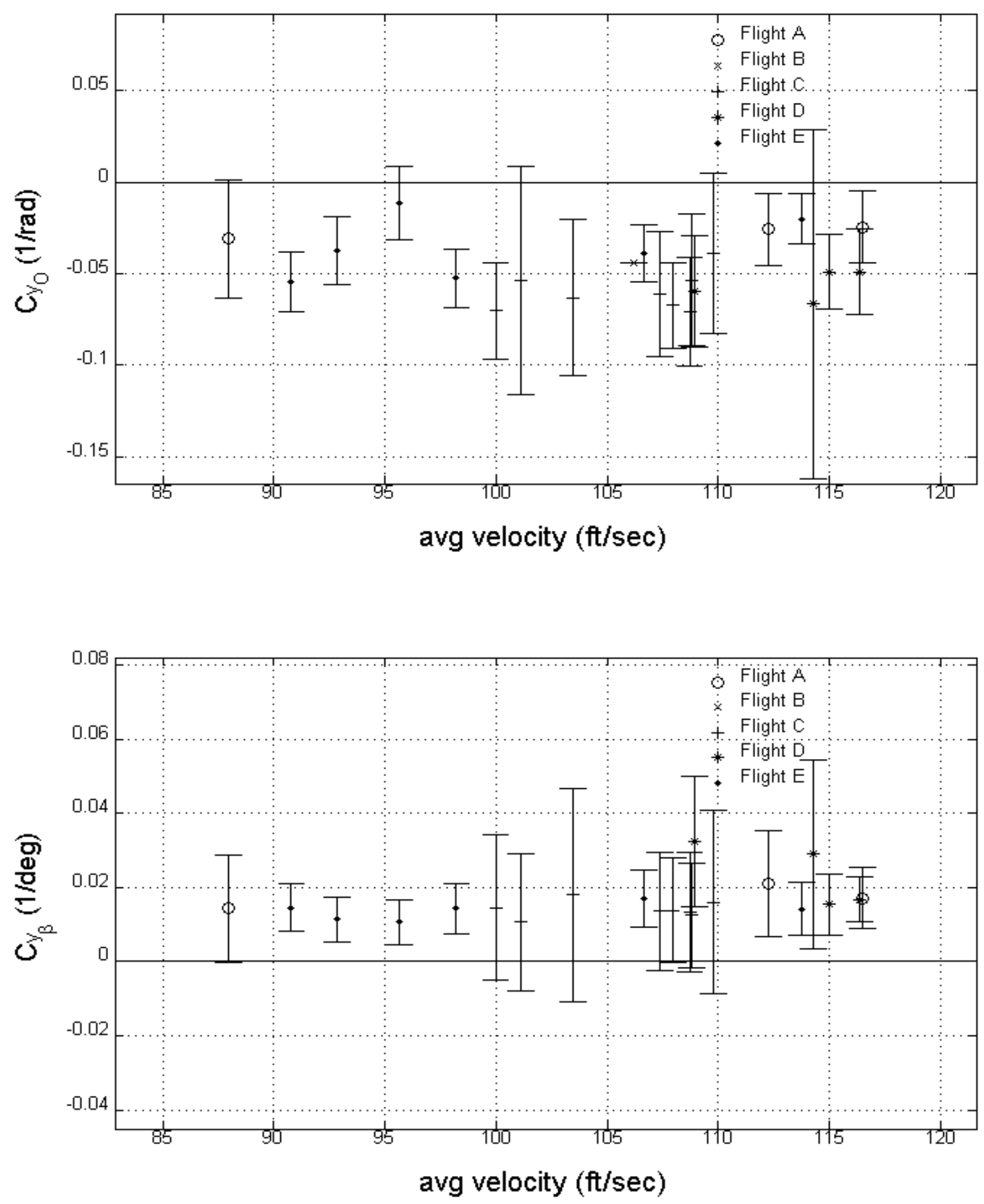

Figure 6.4.50 Off-line estimation results of $\mathrm{C}_{\mathrm{y}_{\mathrm{o}}}$ and $\mathrm{C}_{\mathrm{y}_{\beta}}$ for lateral-directional Flights $\mathrm{A}, \mathrm{B}, \mathrm{C}, \mathrm{D}$, and $\mathrm{E}$ versus velocity (minus selected $\delta_{\mathrm{r}}$ inputs) 

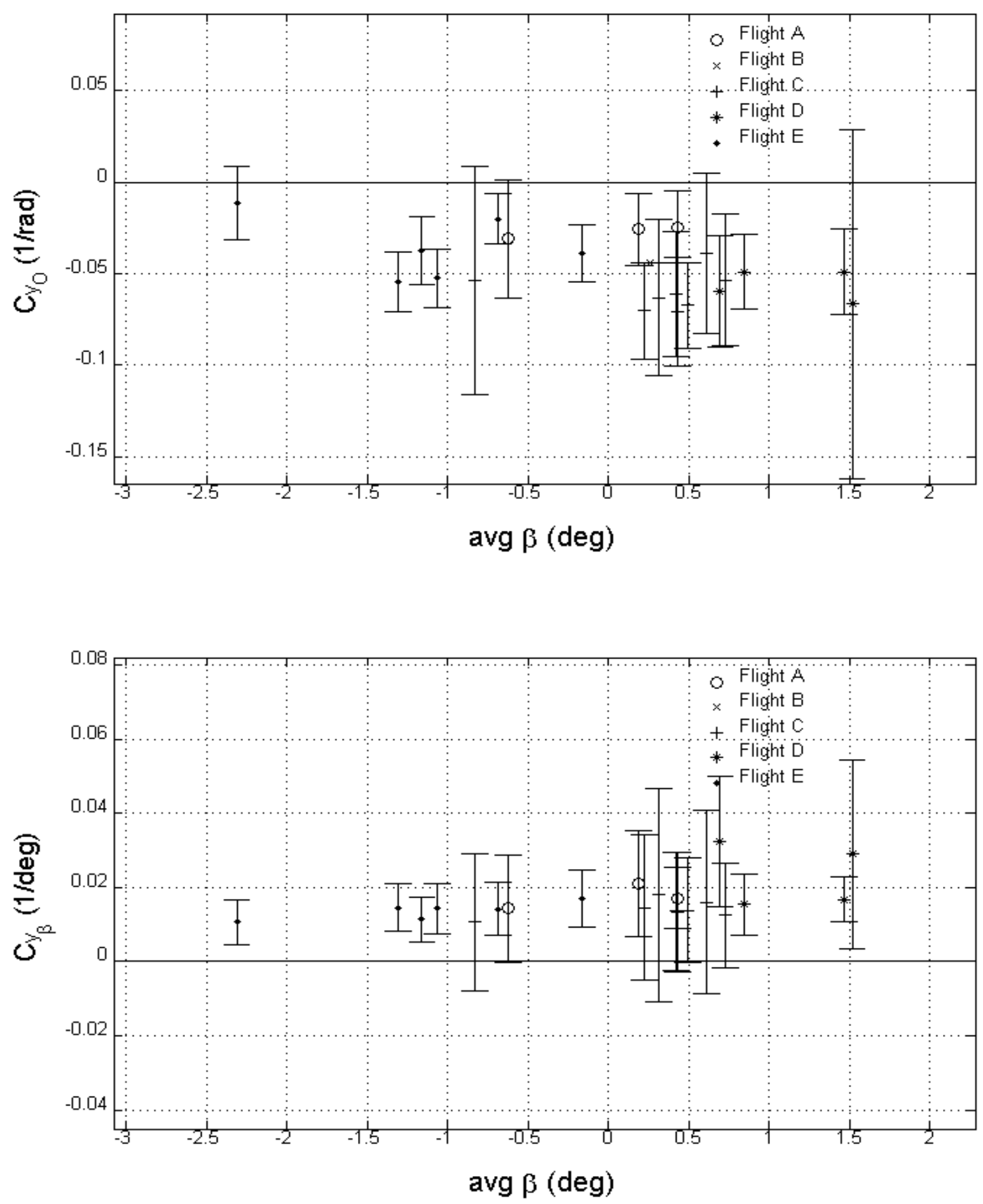

Figure 6.4.51 Off-line estimation results of $\mathrm{C}_{\mathrm{y}_{\mathrm{o}}}$ and $\mathrm{C}_{\mathrm{y} \beta}$ for lateral-directional Flights $\mathrm{A}, \mathrm{B}, \mathrm{C}, \mathrm{D}$, and $\mathrm{E}$ versus sideslip angle (minus selected $\delta_{\mathrm{r}}$ inputs) 

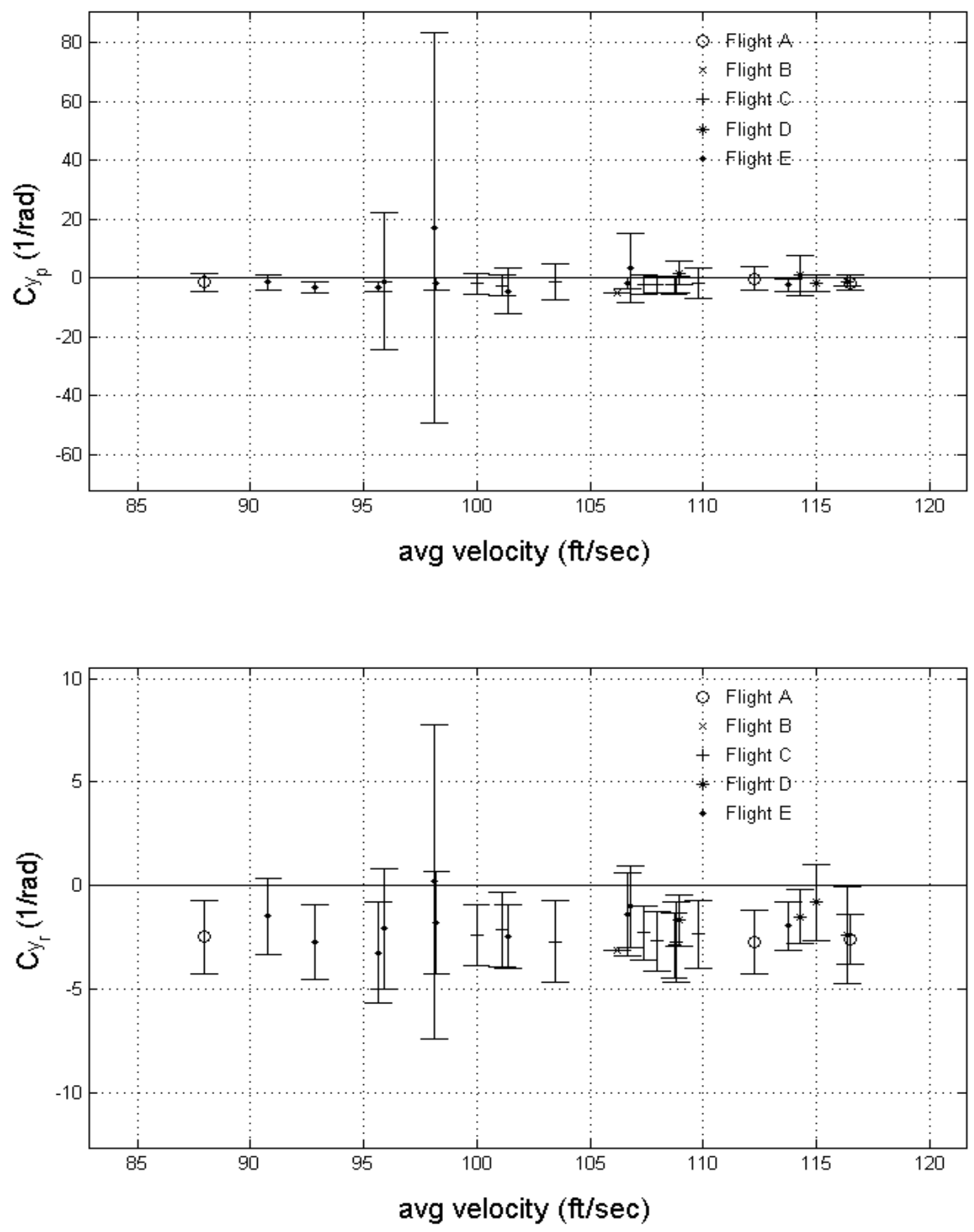

Figure 6.4.52 Off-line estimation results of $\mathrm{C}_{\mathrm{y}_{\mathrm{p}}}$ and $\mathrm{C}_{\mathrm{y}_{\mathrm{r}}}$ for lateral-directional Flights A, B, C, D, and E versus velocity 

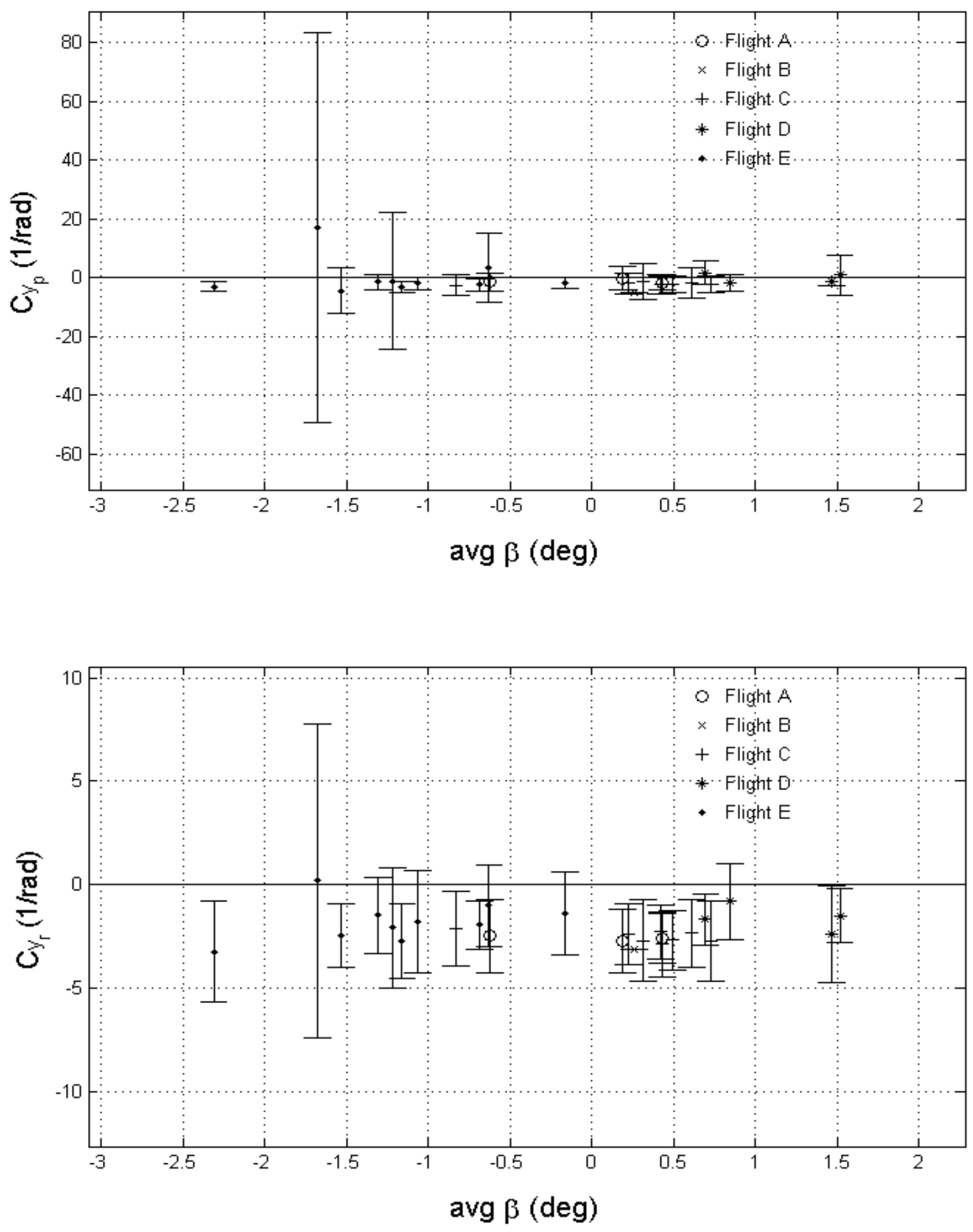

Figure 6.4.53 Off-line estimation results of $\mathrm{C}_{\mathrm{y}_{\mathrm{p}}}$ and $\mathrm{C}_{\mathrm{y}_{\mathrm{r}}}$ for lateral-directional Flights A, B, C, D, and E versus sideslip angle 

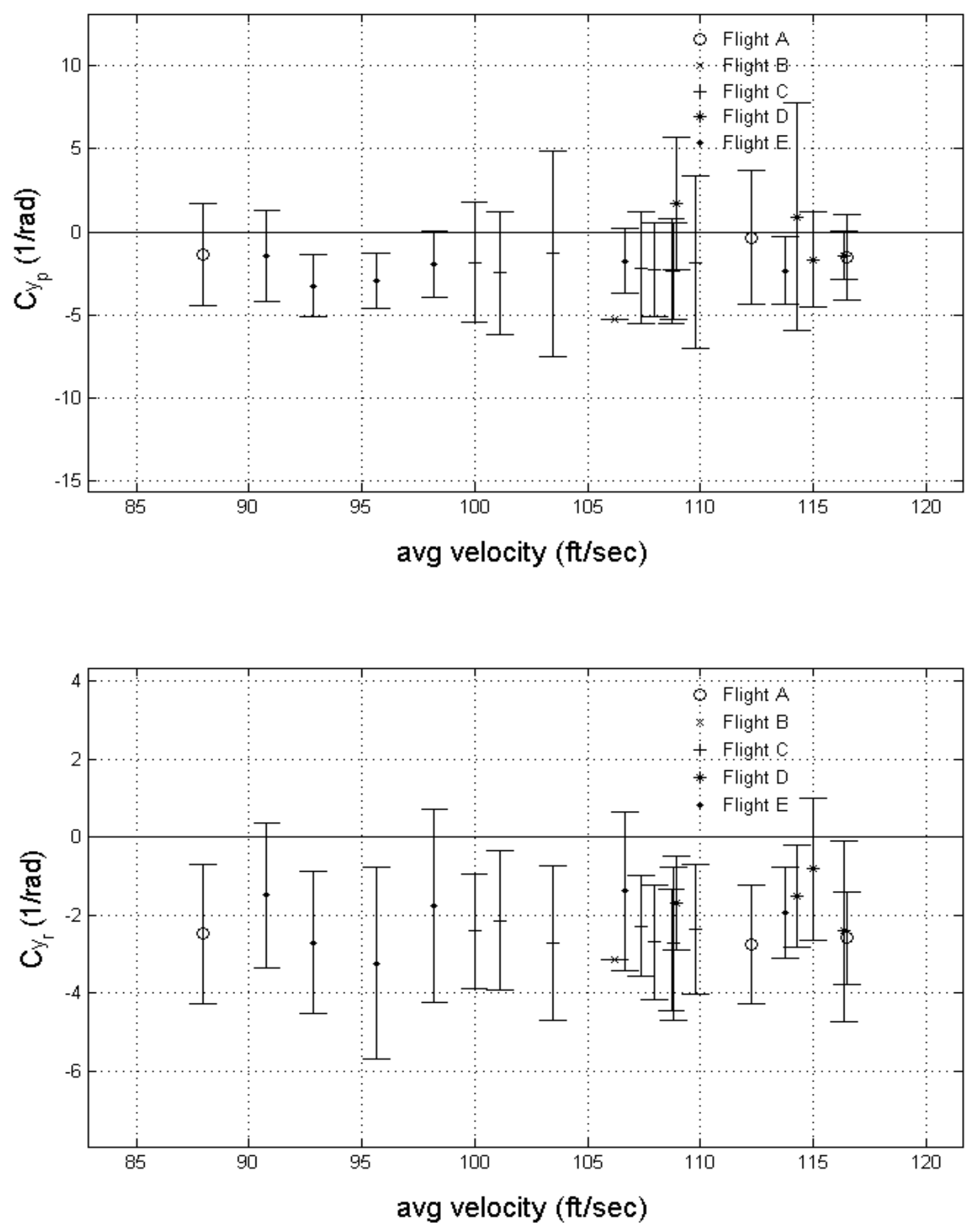

Figure 6.4.54 Off-line estimation results of $\mathrm{C}_{\mathrm{y}_{\mathrm{p}}}$ and $\mathrm{C}_{\mathrm{y}_{\mathrm{r}}}$ for lateral-directional Flights A, B, C, D, and E versus velocity (minus selected $\delta_{\mathrm{r}}$ inputs) 

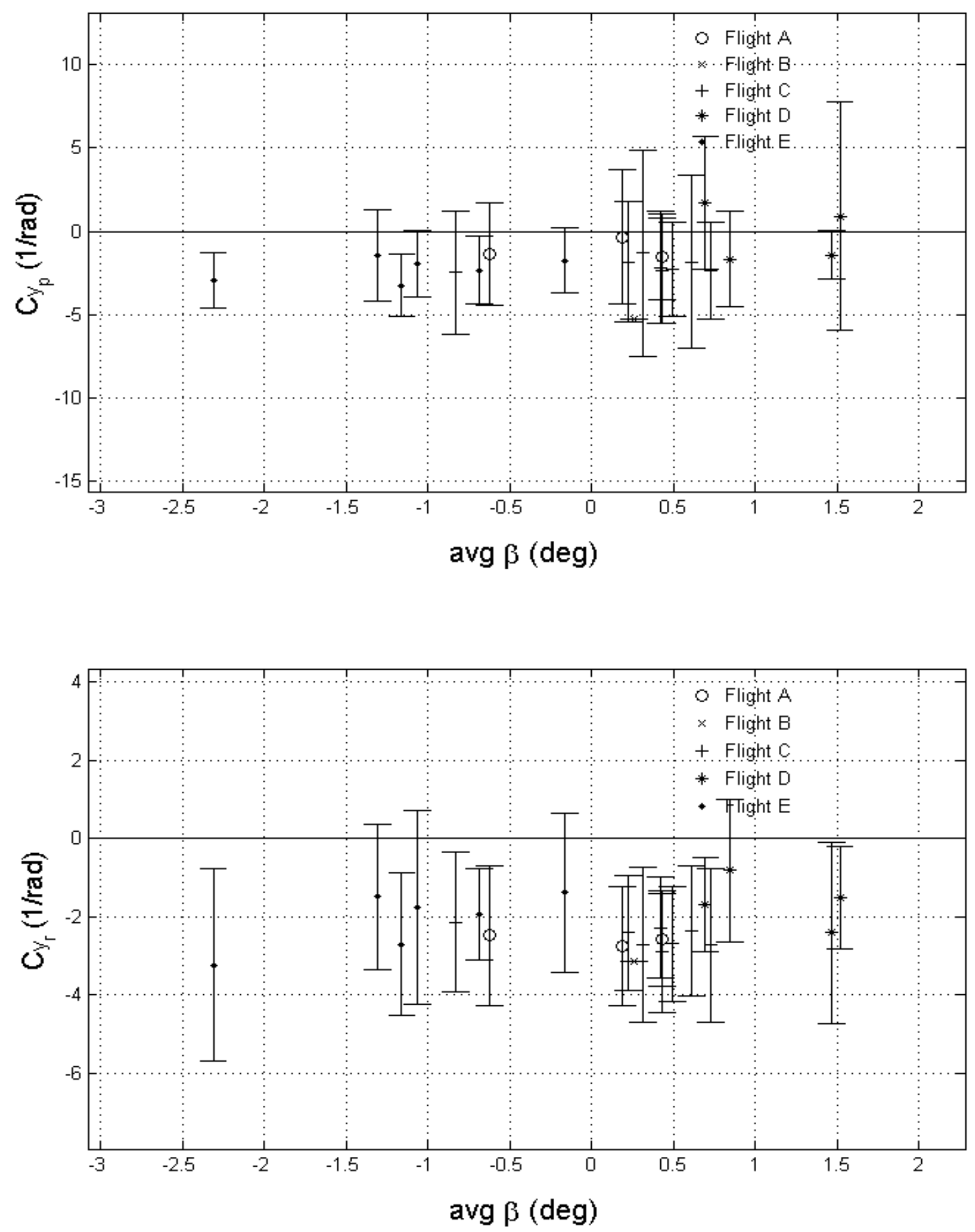

Figure 6.4.55 Off-line estimation results of $\mathrm{C}_{y_{p}}$ and $C_{y_{r}}$ for lateral-directional Flights A, B, C, D, and E versus sideslip angle (minus selected $\delta_{\mathrm{r}}$ inputs) 

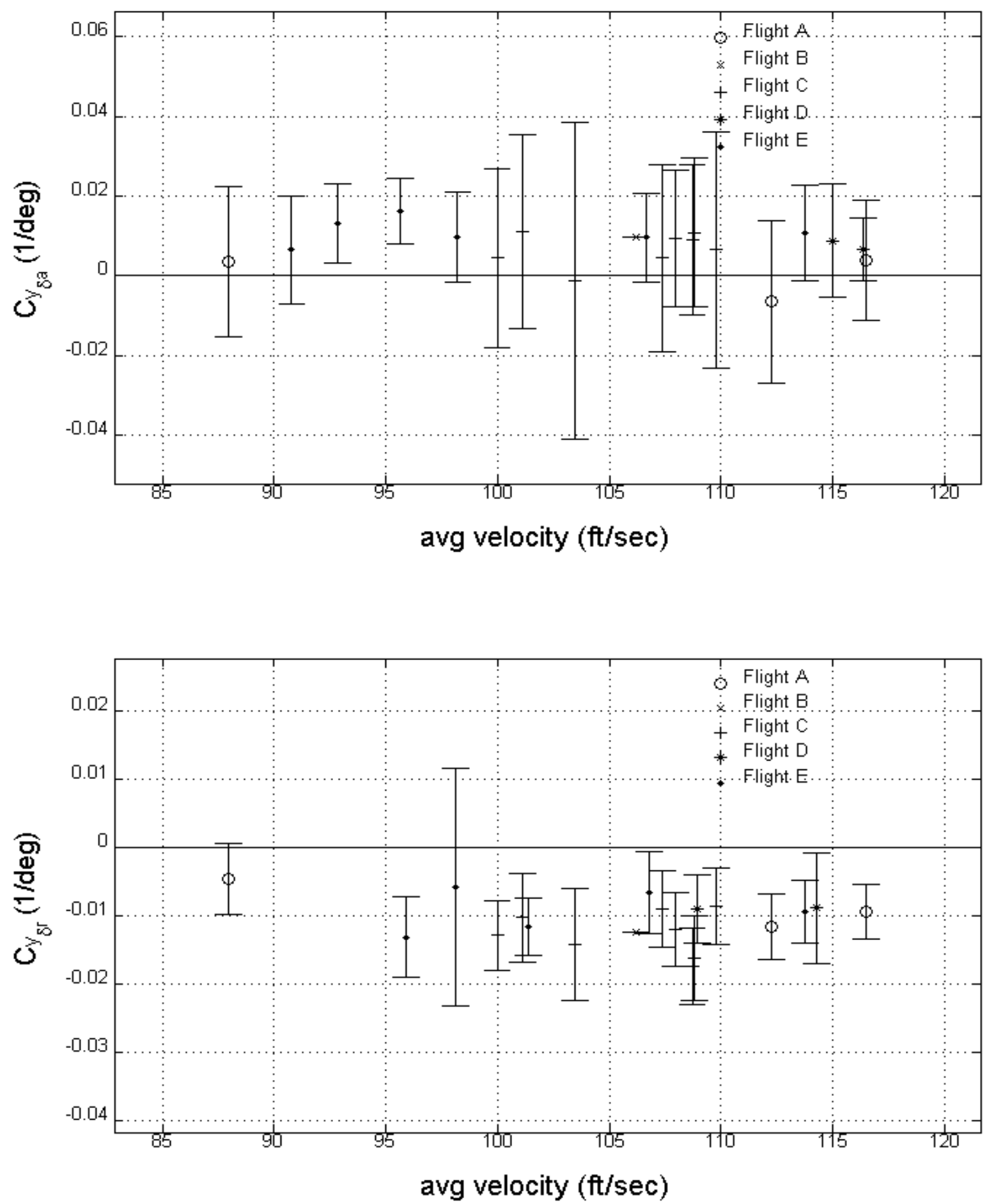

Figure 6.4.56 Off-line estimation results of $\mathrm{C}_{\mathrm{y} \delta_{\mathrm{a}}}$ and $\mathrm{C}_{\mathrm{y} \delta_{\mathrm{r}}}$ for lateral-directional Flights A, B, C, D, and E versus velocity 

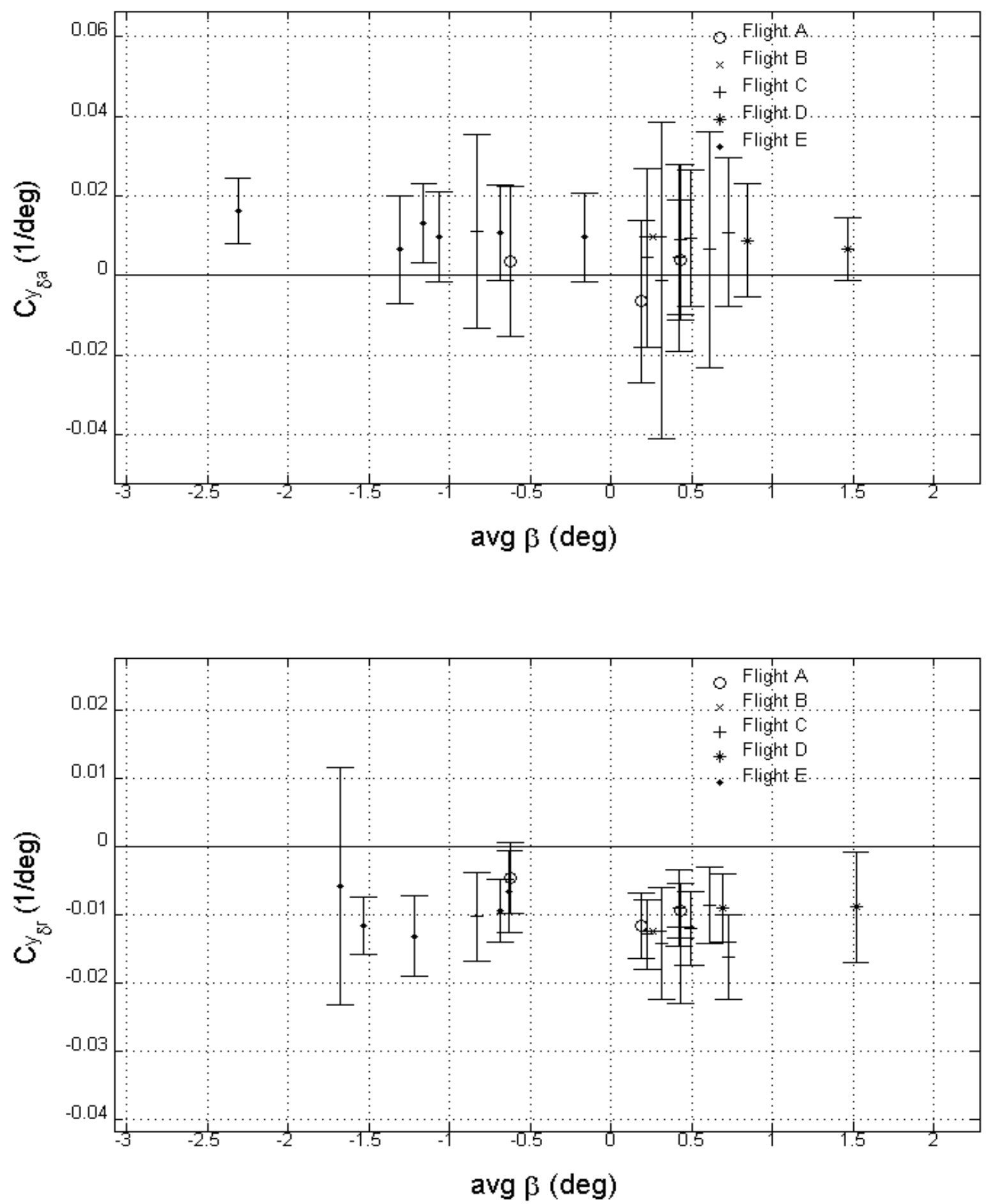

Figure 6.4.57 Off-line estimation results of $\mathrm{C}_{\mathrm{y}_{\mathrm{a}}}$ and $\mathrm{C}_{\mathrm{y} \delta_{\mathrm{r}}}$ for lateral-directional Flights A, B, C, D, and E versus sideslip angle 

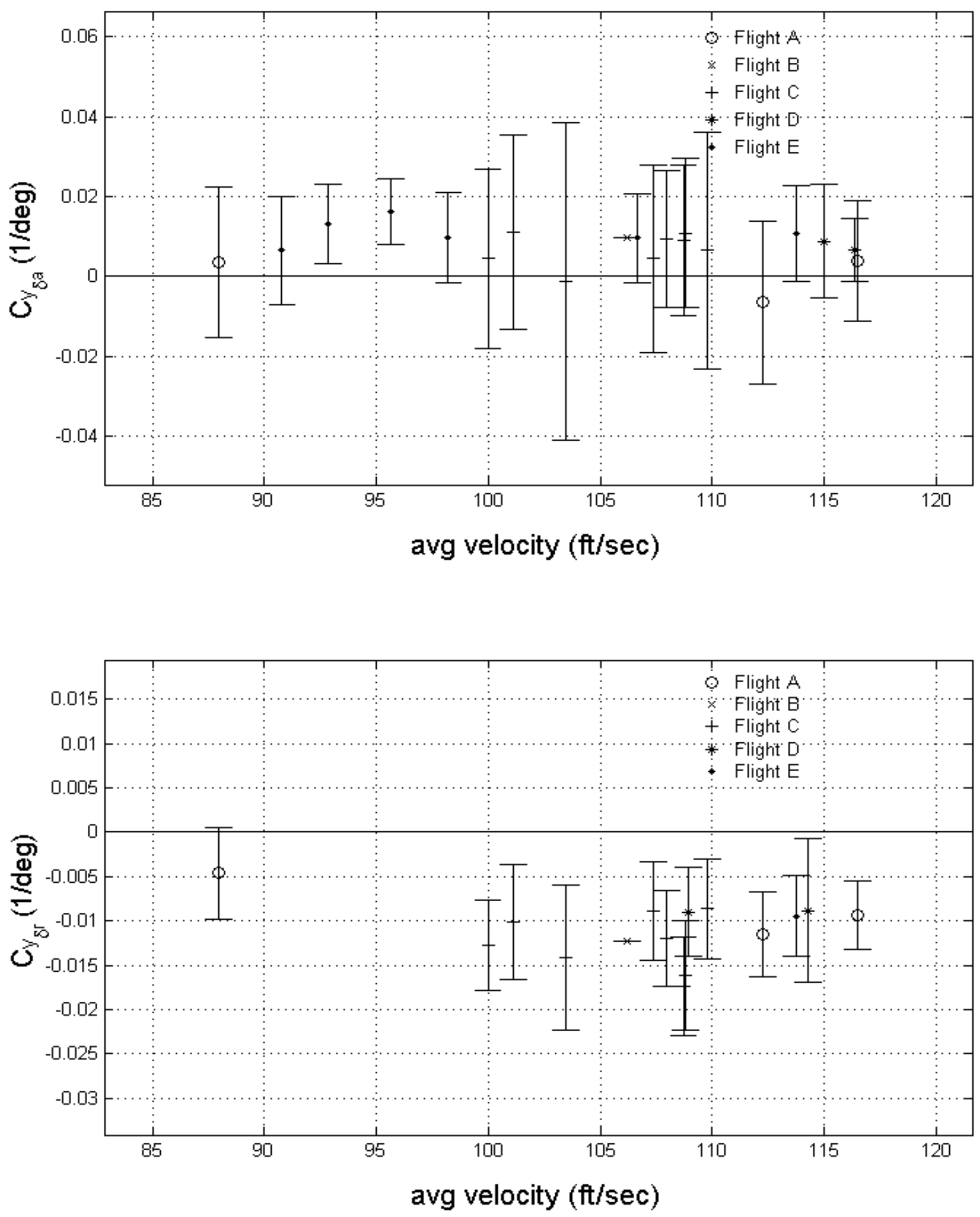

Figure 6.4.58 Off-line estimation results of $\mathrm{C}_{\mathrm{y}_{\mathrm{a}}}$ and $\mathrm{C}_{\mathrm{y} \delta_{\mathrm{r}}}$ for lateral-directional Flights $\mathrm{A}, \mathrm{B}, \mathrm{C}, \mathrm{D}$, and $\mathrm{E}$ versus velocity (minus selected $\delta_{\mathrm{r}}$ inputs) 

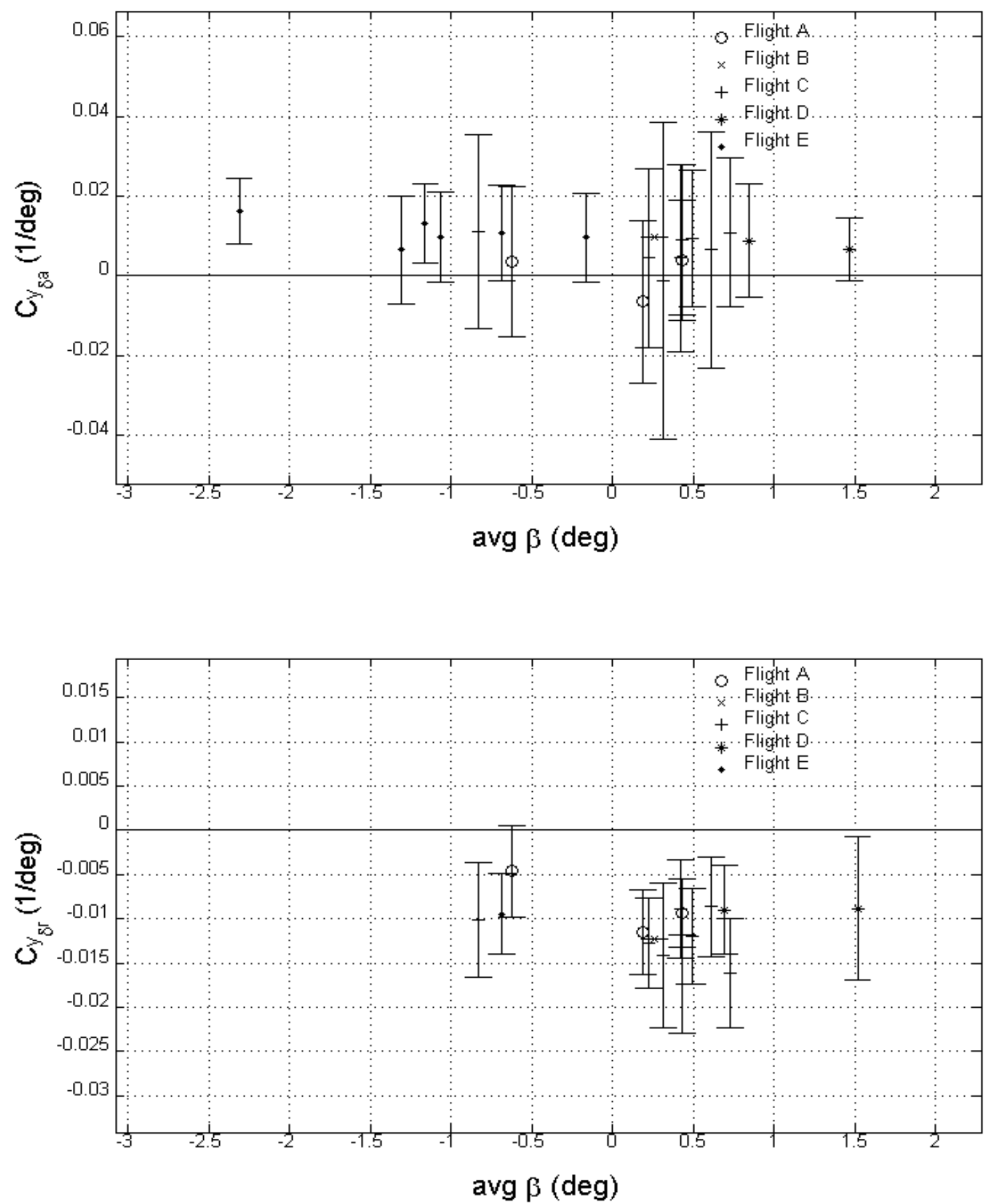

Figure 6.4.59 Off-line estimation results of $\mathrm{C}_{\mathrm{y}_{\mathrm{a}}}$ and $\mathrm{C}_{\mathrm{y} \delta_{\mathrm{r}}}$ for lateral-directional Flights A, B, C, D, and E versus sideslip angle (minus selected $\delta_{\mathrm{r}}$ inputs) 


\section{Chapter 7}

\section{State-Variable Modeling of the Aircraft Dynamics}

\subsection{Theoretical Buildup}

The aircraft dynamics can be approximated by a set of linear small-perturbation equations of motion within a state-matrix format;

$$
\begin{aligned}
& \dot{x}=A x+B u \\
& y=C x+D u
\end{aligned}
$$

where " $x$ " is considered the aircraft state vector, " $u$ " is the aircraft control vector and " $y$ " is the aircraft output vector. The average pEst coefficients resulting from the ML method were inserted into a set of standard decoupled linear equations of an aircraft's longitudinal and lateral-directional dynamics to create a linear model. This model was then compared to two other linear models. The first model was obtained with a Batch Least Squares (BLS) technique, and the second model was obtained by using a subspacebased technique found in the system identification toolbox from Matlab. The following sections will review and compare the various techniques for all three of the linear models created. For PID analysis the longitudinal simulation case used Flight 17 and the lateral case used Flight $\mathrm{C}$ respectively. For validation purposes Flight 18 and Flight $\mathrm{E}$ were then used respectively. 


\subsection{BLS and System Identification Toolbox Linear Models}

\section{Model \#1: Background}

The BLS technique is a widely used approach for estimation of vector parameters from related input-output data and essentially involves solving an over-determined linear system in the least squares sense. Based on linear algebra, this approach leads to a simple formulation and a straightforward analysis, allowing the use of powerful and well-known algorithms. The general linear regression model is given by:

$$
Y=X \beta+\varepsilon
$$

where $Y$ is a $(n \times 1)$ vector of known responses of the system, $X$ is a $(n \times p)$ matrix of known inputs to the system (note that the last column of this matrix is usually a column of ones allowing for a "bias" - namely a constant input to the system - to be introduced), $\beta$ in the $(\mathrm{p} \times 1)$ vector of parameters to be estimated, and $\varepsilon$ is a $(\mathrm{n} \times 1)$ vector of independent normal random variables, with zero mean $(E\{\varepsilon\}=0)$ and unknown diagonal variancecovariance matrix. This matrix is generally assumed to be a multiple of the $(n \times n)$ identity matrix: $\left(\sigma^{2}\{\varepsilon\}=\sigma^{2} I\right)$. Therefore we have that $E\{Y\}=X \beta$ and $\sigma^{2}\{Y\}=\sigma^{2}$ I. The problem is to find the vector $\beta$ such that $\mathrm{X} \beta$ (which is the expected value of $\mathrm{Y}$ ) is as close as possible (in the least squares sense) to $\mathrm{Y}$, so that $\sigma^{2}$ is minimized. Particularly, the objective is to find the value of $\beta$ that minimizes the following quadratic index:

$$
Q=\varepsilon^{T} \varepsilon=(Y-X \beta)^{T}(Y-X \beta)
$$

The solution to this problem is given by:

$$
b=\left(X^{\mathrm{T}} \mathrm{X}\right)^{-1} \mathrm{X}^{\mathrm{T}} \mathrm{Y}
$$

It can be shown - using the Gauss-Markov theorem - that this solution is such that the error vector:

$$
e=Y-X b
$$

has zero mean - meaning unbiased estimation - and minimum variance among all the possible linear unbiased solutions. Using terms from statistics the relative estimation is known as BLUE (Best Linear Unbiased Estimation). Furthermore, it can be shown that the resulting estimation for $\sigma^{2}$ is the Mean Square Error (MSE):

$$
M S E=\frac{e^{T} e}{n-p}
$$


The covariance of the solution is:

$$
\begin{aligned}
\sigma^{2}\{b\} & =E\left\{(b-E\{b\})(b-E\{b\})^{T}\right\}=\left(X^{T} X\right)^{-1} X^{T} \sigma^{2}\{Y\} X\left(X^{T} X\right)^{-1} \\
& =\sigma^{2}\left(X^{T} X\right)^{-1}
\end{aligned}
$$

Substituting the MSE in lieu of $\sigma^{2}$ in equation (1.6) we obtain:

$$
\sigma^{2}\{b\}=\frac{e^{T} e}{n-p}\left(X^{T} X\right)^{-1}
$$

Since the specific problem is the identification of a linear system of the form:

$$
\left[\begin{array}{l}
\dot{x}(t) \\
y(t)
\end{array}\right]=\left[\begin{array}{ll}
A & B \\
C & D
\end{array}\right]\left[\begin{array}{l}
x(t) \\
u(t)
\end{array}\right]
$$

then the variables in (7.1.3):

$$
\begin{gathered}
Y=\left[\begin{array}{ll}
\dot{x}^{T}(t) & y^{T}(t)
\end{array}\right] \\
X=\left[\begin{array}{ll}
x^{T}(t) & u^{T}(t)
\end{array}\right] \\
\beta=\left[\begin{array}{ll}
A & B \\
C & D
\end{array}\right]^{T}
\end{gathered}
$$

\section{Model \#2: Background}

This linear model was obtained using the Matlab functions "n4sid", which provided an initial guess using a subspace based technique and "pem" that provided the first predicated estimation for the general linear model. The "n4sid" Matlab function is a subspace-based method, which does not use an iterative search approach. The "pem" function is a standard prediction error, or also known as a version of the maximum likelihood method, based on an iterative minimization.

\section{Identification Process:}

The actual identification for models one and two were performed in a two step process. For step one, the selected longitudinal or lateral-directional flight data time histories were introduced to a set of Simulink schemes, Figures 7.2.1-7.2.2, which essentially provided the rearrangement of the aircraft signals. 


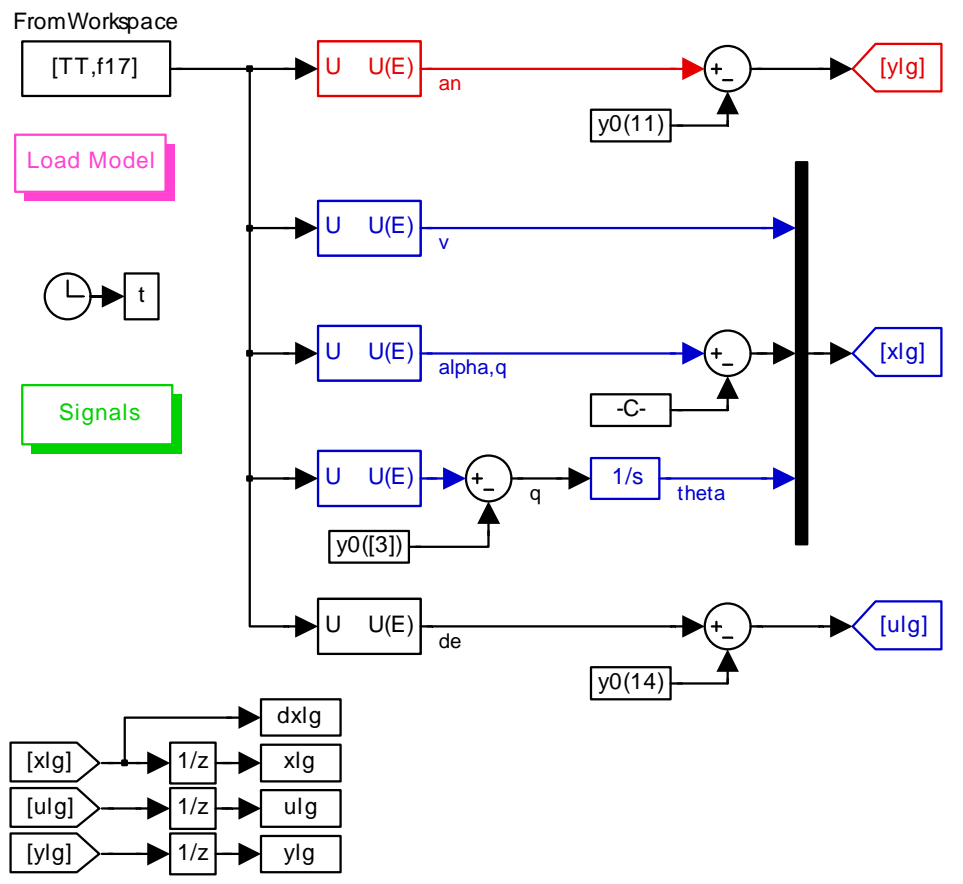

Figure 7.2.1 Setup scheme of longitudinal state-space matrices

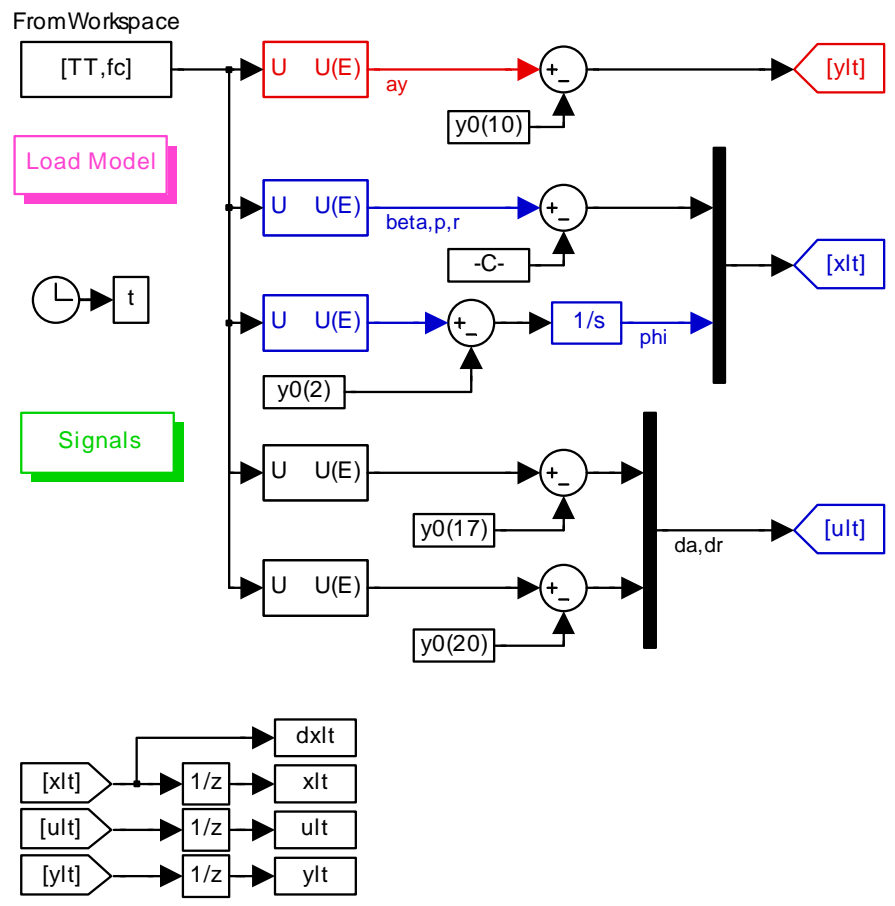

Figure 7.2.2 Setup scheme of lateral-directional state space matrices 
For step two, a batch Matlab file performed the BLS method (Model \#1) and identification toolbox method (Model \#2) on the data rearranged from the previously shown Simulink schemes. Model one, resulting from the BLS algorithm, a decoupled linear model that has the following shape:

For the longitudinal dynamics;

Model \#1 (BLS):

$$
\begin{gathered}
{\left[\begin{array}{c}
\dot{\alpha} \\
\dot{q}
\end{array}\right]=\left[\begin{array}{cc}
-0.9895 & 0.1755 \\
-2.7477 & -9.1748
\end{array}\right]\left[\begin{array}{l}
\alpha \\
q
\end{array}\right]+\left[\begin{array}{l}
-1.0206 \\
-40.8586
\end{array}\right] \delta_{e}} \\
{\left[\begin{array}{c}
A z \\
\alpha \\
q
\end{array}\right]=\left[\begin{array}{cc}
0.0545 & 0.0476 \\
1.0000 & 0 \\
0 & 1.0000
\end{array}\right]\left[\begin{array}{l}
\alpha \\
q
\end{array}\right]+\left[\begin{array}{c}
0.0806 \\
0 \\
0
\end{array}\right] \delta_{e}}
\end{gathered}
$$

and for the lateral-directional dynamics;

Model \#1 (BLS):

$$
\begin{aligned}
& {\left[\begin{array}{l}
\dot{\beta} \\
\dot{p} \\
\dot{r}
\end{array}\right]=\left[\begin{array}{lll}
3.0547 & 0.3796 & -0.9095 \\
-59.7582 & -8.7077 & 5.5280 \\
11.6000 & -3.6886 & -9.0277
\end{array}\right]\left[\begin{array}{l}
\beta \\
p \\
r
\end{array}\right]+\left[\begin{array}{cc}
-3.1822 & -1.1393 \\
79.7854 & 7.0154 \\
16.8485 & -40.3067
\end{array}\right]\left[\begin{array}{l}
\delta_{a} \\
\delta_{r}
\end{array}\right]} \\
& {\left[\begin{array}{l}
A_{y} \\
\beta \\
p \\
r
\end{array}\right]=\left[\begin{array}{ccc}
0.0333 & -0.0016 & -0.0024 \\
1.0000 & 0 & 0 \\
0 & 1.0000 & 0 \\
0 & 0 & 1.0000
\end{array}\right]\left[\begin{array}{l}
\beta \\
p \\
r
\end{array}\right]+\left[\begin{array}{cc}
0.0071 & -0.0075 \\
0 & 0 \\
0 & 0 \\
0 & 0
\end{array}\right]\left[\begin{array}{l}
\delta_{a} \\
\delta_{r}
\end{array}\right]}
\end{aligned}
$$

Originally a "full" case version included velocity and $\theta$ for the longitudinal dynamics and $\phi$ for the lateral dynamics respectively, but no improvements were observed from this version.

Model two provided from the Matlab identification toolbox was decoupled into longitudinal and lateral-directional dynamics as shown in model one. The longitudinal case was made up of 18 internal states, one input $\left(\delta_{e}\right)$, and three outputs $A_{n}, \alpha$, and q. The lateral case was made up of 11 internal states, two inputs $\left(\delta_{\mathrm{a}}, \delta_{\mathrm{r}}\right)$, and four outputs $A_{y}, \beta, p$ and $r$. Matrices obtained from this technique were not shown due to their size and complexity. 


\subsection{Linear Model}

\section{$\underline{\text { Model \#3 }}$}

For creating the third model, the procedure involved the state variable modeling of the aircraft dynamics using the ML estimates obtained from flight data. An average value for each longitudinal and lateral-directional coefficient, obtained from available flight data, was used in computing the final set of model four matrices. Again a general description of the state variable modeling of the aircraft dynamics can be described by a set of linear small-perturbation equations of motion within a state-matrix format equations (7.1.1) and (7.1.2) with the following basic assumptions in mind;

\section{Rigid body aircraft}

2. Earth is an inertial-enough reference frame

3. Aircraft mass and mass distributions are constant

4. $\mathrm{XZ}$ is a plane of symmetry

5. Negligible gyroscopic effects for the engine

6. Equations are derived with respect to the stability axes

7. Small perturbations

8. Only 3 primary control surfaces: elevator(s), aileron(s), and rudder

Separation between longitudinal and lateral directional dynamics, leads to:

$$
\begin{aligned}
& \dot{x}_{\text {Long }}=A_{\text {Long }} x_{\text {Long }}+B_{\text {Long }} u_{\text {Long }} \\
& y_{\text {Long }}=C_{\text {Long }} x_{\text {Long }}+D_{\text {Long }} u_{\text {Long }}
\end{aligned}
$$

where the longitudinal state, control inputs and outputs are represented as

$$
x_{\text {Long }}=\{\alpha, u, q, \theta\}^{T}, u_{\text {Long }}=\left\{\delta_{E}\right\}, y_{\text {Long }}=\left\{a_{Z}, \alpha, u, q, \theta\right\}^{T}
$$

and for the lateral-direction:

$$
\begin{aligned}
& \dot{x}_{\text {LatDir }}=A_{\text {LatDir }} x_{\text {LatDir }}+B_{\text {LatDir }} u_{\text {LatDir }} \\
& y_{\text {LatDir }}=C_{\text {LatDir }} x_{\text {LatDir }}+D_{\text {LatDir }} u_{\text {LatDir }}
\end{aligned}
$$

where the longitudinal state, control inputs and outputs are represented as

$$
x_{\text {LatDir }}=\{\beta, p, r, \phi\}^{T}, u_{\text {LatDir }}=\left\{\delta_{A}, \delta_{R}\right\}, y_{\text {LatDir }}=\left\{a_{y}, \beta, p, r, \phi\right\}^{T}
$$


The longitudinal state equations can therefore be written in the following format, equation (7.3.7),

$$
\left\{\begin{array}{c}
\dot{\alpha} \\
\dot{u} \\
\dot{q} \\
\dot{\theta}
\end{array}\right\}=\left[\begin{array}{cccc}
Z_{\alpha}^{\prime} & Z_{u}^{\prime} & Z_{q}^{\prime} & Z_{\theta}^{\prime} \\
X_{\alpha}^{\prime} & X_{u}^{\prime} & X_{q}^{\prime} & X_{\theta}^{\prime} \\
M_{\alpha}^{\prime} & M_{u}^{\prime} & M_{q}^{\prime} & M_{\theta}^{\prime} \\
0 & 0 & 1 & 0
\end{array}\right]\left\{\begin{array}{l}
\alpha \\
u \\
q \\
\theta
\end{array}\right\}+\left[\begin{array}{c}
Z_{\delta E}^{\prime} \\
X_{\delta E}^{\prime} \\
M_{\delta E}^{\prime} \\
0
\end{array}\right]\left\{\delta_{E}\right\}
$$

where the elements of the $\mathrm{A}$ and $\mathrm{B}$ matrices are related to the standard aircraft longitudinal dimensional derivatives found in Appendix A. The lateral directional state equations are written in the following format, equation (7.3.8),

$$
\left\{\begin{array}{c}
\dot{\beta} \\
\dot{p} \\
\dot{r} \\
\dot{\phi}
\end{array}\right\}=\left[\begin{array}{cccc}
Y_{\beta}^{\prime} & Y_{p}^{\prime} & Y_{r}^{\prime} & Y_{\phi}^{\prime} \\
L_{\beta}^{\prime} & L_{p}^{\prime} & L_{r}^{\prime} & L_{\phi}^{\prime} \\
N_{\beta}^{\prime} & N_{p}^{\prime} & N_{r}^{\prime} & N_{\phi}^{\prime} \\
0 & 1 & \tan \Theta_{1} & 0
\end{array}\right]\left\{\begin{array}{l}
\beta \\
p \\
r \\
\phi
\end{array}\right\}+\left[\begin{array}{cc}
Y_{\delta A}^{\prime} & Y_{\delta R}^{\prime} \\
L_{\delta A}^{\prime} & L_{\delta R}^{\prime} \\
N_{\delta A}^{\prime} & N_{\delta R}^{\prime} \\
0 & 0
\end{array}\right]\left\{\begin{array}{l}
\delta_{A} \\
\delta_{R}
\end{array}\right\}
$$

where the elements of the A and B matrices are related to the standard aircraft lateraldirectional dimensional derivatives, also found in Appendix A. The general longitudinal output equations are defined as:

$$
\left\{\begin{array}{c}
a_{z} \\
\alpha \\
u \\
q \\
\theta
\end{array}\right\}=\left[\begin{array}{cccc}
Z_{\alpha}^{\prime \prime} & Z_{u}^{\prime \prime} & Z_{q}^{\prime \prime} & Z_{\theta}^{\prime \prime} \\
1 & 0 & 0 & 0 \\
0 & 1 & 0 & 0 \\
0 & 0 & 1 & 0 \\
0 & 0 & 0 & 1
\end{array}\right]\left\{\begin{array}{l}
\alpha \\
u \\
q \\
\theta
\end{array}\right\}+\left[\begin{array}{c}
Z_{\delta E}^{\prime \prime} \\
0 \\
0 \\
0 \\
0
\end{array}\right]\left\{\delta_{E}\right\}
$$

and the general lateral-directional outputs are defined as:

$$
\left\{\begin{array}{l}
a_{y} \\
\beta \\
p \\
r \\
\phi
\end{array}\right\}=\left[\begin{array}{cccc}
Y_{\beta}^{\prime \prime} & Y_{p}^{\prime \prime} & Y_{r}^{\prime \prime} & Y_{\phi}^{\prime \prime} \\
1 & 0 & 0 & 0 \\
0 & 1 & 0 & 0 \\
0 & 0 & 1 & 0 \\
0 & 0 & 0 & 1
\end{array}\right]\left\{\begin{array}{l}
\beta \\
p \\
r \\
\phi
\end{array}\right\}+\left[\begin{array}{cc}
Y_{\delta A}^{\prime \prime} & Y_{\delta R}^{\prime \prime} \\
0 & 0 \\
0 & 0 \\
0 & 0 \\
0 & 0
\end{array}\right]\left\{\begin{array}{l}
\delta_{A} \\
\delta_{R}
\end{array}\right\}
$$

A full description of the state variable modeling equations used to create model three are provided in Appendix A. Using the formulas in Appendix A, to compute all terms in 
equations 7.3.7 thru 7.3.10, the average pEst coefficients (obtained from sections 6.3 and 6.4 results) were then used to produce the following models:

For the longitudinal dynamics;

Model \#3 (pEst):

$$
\begin{aligned}
& {\left[\begin{array}{c}
\dot{\alpha} \\
\dot{V} \\
\dot{q} \\
\dot{\theta}
\end{array}\right]=\left[\begin{array}{cccc}
-3.8349 & 0 & 1.0333 & 0 \\
-6.3432 & 0 & 0 & -32.2000 \\
-8.8331 & 0 & -4.5214 & 0 \\
0 & 0 & 1.0000 & 0
\end{array}\right]\left[\begin{array}{l}
\alpha \\
V \\
q \\
\theta
\end{array}\right]+\left[\begin{array}{c}
-0.3279 \\
52.3719 \\
-28.7463 \\
0
\end{array}\right] \delta_{e}} \\
& {\left[\begin{array}{c}
A_{n} \\
\alpha \\
q
\end{array}\right]=\left[\begin{array}{cccc}
-357.5706 & 0 & 3.1023 & 0 \\
1.0000 & 0 & 0 & 0 \\
0 & 0 & 1.0000 & 0
\end{array}\right]\left[\begin{array}{l}
\alpha \\
V \\
q \\
\theta
\end{array}\right]+\left[\begin{array}{c}
-30.5766 \\
0 \\
0
\end{array}\right] \delta_{e}}
\end{aligned}
$$

For the lateral-directional dynamics;

Model \#3 (pEst):

$$
\begin{gathered}
{\left[\begin{array}{c}
\dot{\beta} \\
\dot{p} \\
\dot{r} \\
\dot{\varphi}
\end{array}\right]=\left[\begin{array}{cccc}
0.8157 & -0.0701 & -1.0893 & 0.3050 \\
-82.3277 & -11.5602 & 5.0121 & 0 \\
19.9477 & -1.4447 & -2.6382 & 0 \\
0 & 1.0000 & -0.0069 & 0
\end{array}\right]\left[\begin{array}{l}
\beta \\
p \\
r \\
\varphi
\end{array}\right]+\left[\begin{array}{cc}
0.3558 & -0.3996 \\
102.0631 & 2.5918 \\
5.5099 & -12.6008 \\
0 & 0
\end{array}\right]\left[\begin{array}{l}
\delta_{a} \\
\delta_{r}
\end{array}\right]} \\
{\left[\begin{array}{c}
A y \\
\beta \\
p \\
r
\end{array}\right]=\left[\begin{array}{cccc}
86.1199 & -7.3964 & -9.4236 & 0 \\
1.0000 & 0 & 0 & 0 \\
0 & 1.0000 & 0 & 0 \\
0 & 0 & 1.0000 & 0
\end{array}\right]\left[\begin{array}{l}
\beta \\
p \\
r \\
\varphi
\end{array}\right]+\left[\begin{array}{cc}
37.5647 & -42.1875 \\
0 & 0 \\
0 & 0 \\
0 & 0
\end{array}\right]\left[\begin{array}{l}
\delta_{a} \\
\delta_{r}
\end{array}\right]}
\end{gathered}
$$

For all three case models, both the longitudinal and lateral-directional dynamics were then compared with actual measured flight data. 


\subsection{Comparison of Longitudinal and Lateral-Directional Linear Models}

To evaluate the performance of these estimations, a Simulink scheme was designed for both the longitudinal and lateral-directional case. A different set of flight data was then used during the validation purposes. For longitudinal case, Flight 18, and Flight $\mathrm{E}$ for the lateral flight information. These validation schemes are shown in Figures 7.4.1 and 7.4.2. For the validation scheme shown, flight data was inserted from the Matlab workspace to the various plant models.

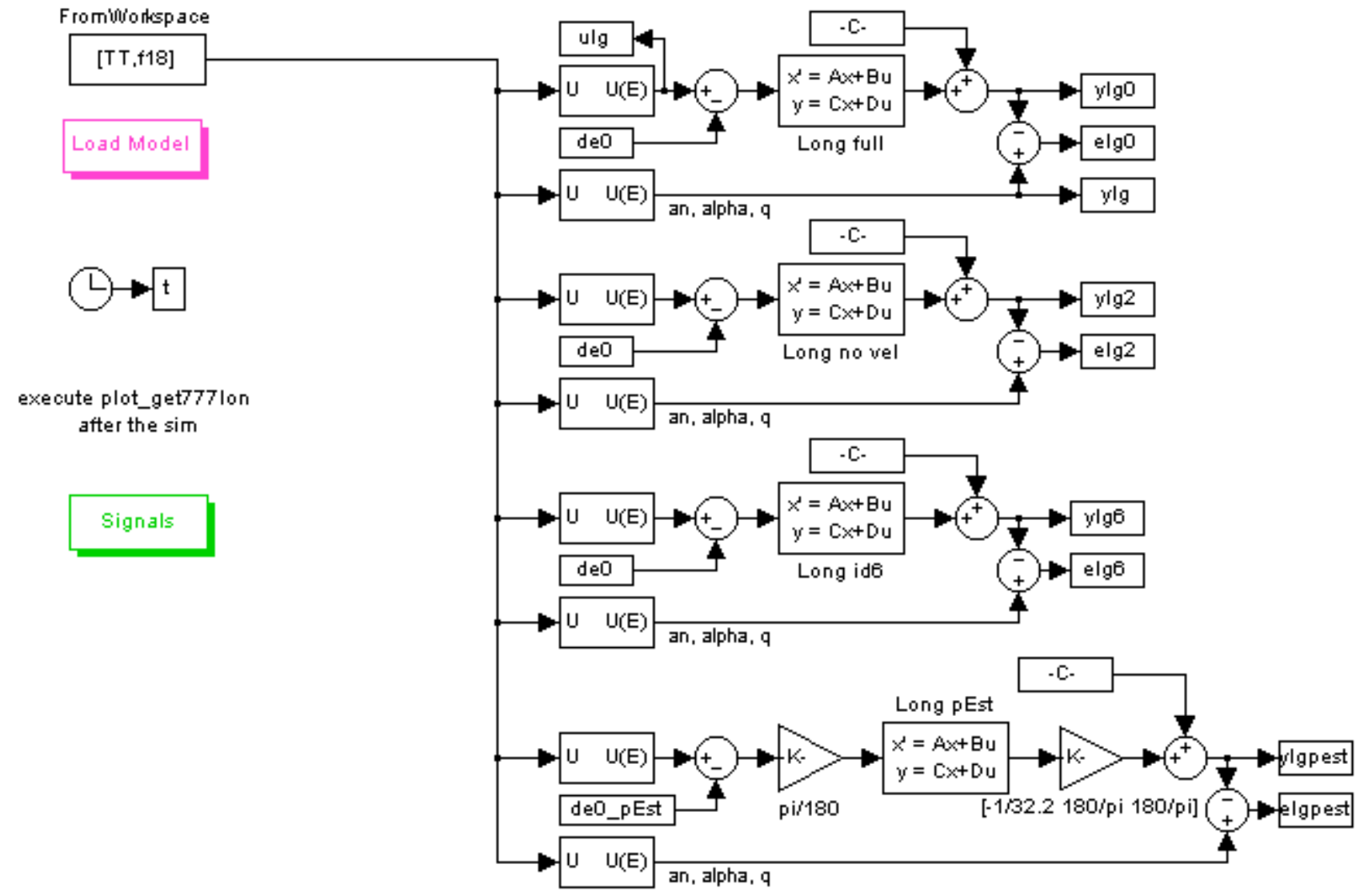

Figure 7.4.1 Simulink validation scheme for longitudinal state-space models

As a note, Model \#1 (BLS method: longitudinal case) is denoted as "no vel" included state and input variables included $\mathrm{a}_{\mathrm{n}}, \alpha, \mathrm{q}$, and $\delta_{\mathrm{e}}$. Model \#2 (identification toolbox) is denoted as "id6". Figure 7.4.2 shows the lateral validation scheme. 


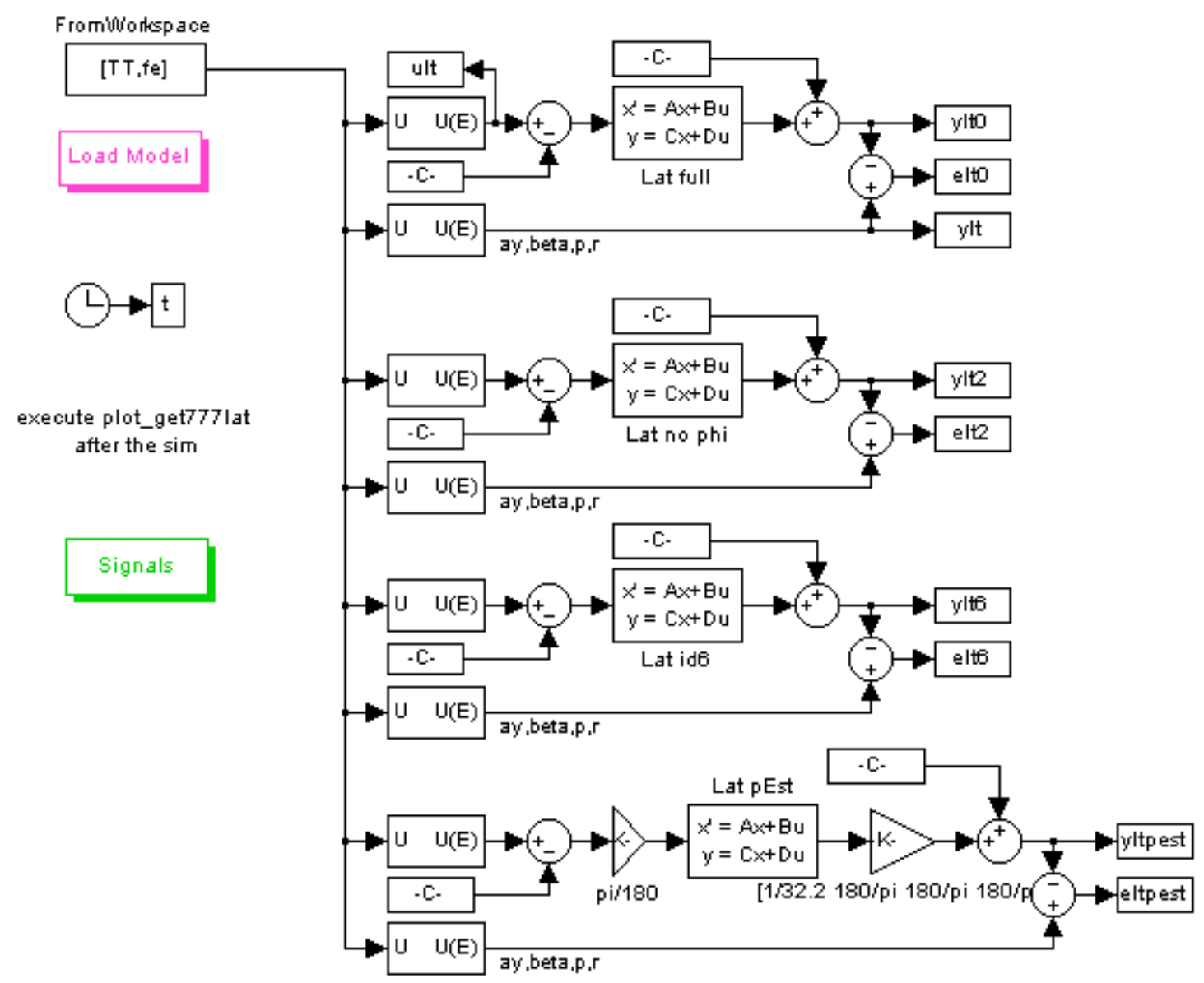

Figure 7.4.2 Simulink validation scheme for lateral-directional state space models

As with the previous scheme, Model \#1 (BLS method: lateral-directional case) is denoted as "no phi" included state and input variables included $\mathrm{a}_{\mathrm{y}}, \beta, \mathrm{p}, \mathrm{r}, \delta_{\mathrm{a}}$, and $\delta_{\mathrm{r}}$. Model \#2 (identification toolbox) is denoted as "id6".

\section{Longitudinal and Lateral-Directional Results}

Figures 7.4.3-7.4.4 show a comparison of the longitudinal state-space results for all three aircraft linear models compared with measured flight data. Overall the matches for $\mathrm{a}_{\mathrm{n}}, \alpha$, and $\mathrm{q}$ were very good throughout the maneuver frame time shown. The best matches of the aircraft responses were obtained from the "id6" and "pEst" models. Figures 7.4.5-7.4.6 show a comparison of the lateral-directional state-space results for all three aircraft linear models compared with measured flight data. For the lateral dynamics, the overall the matches did not fair as well as the results from the longitudinal 
case. The lateral acceleration, $\mathrm{a}_{\mathrm{y}}$, and roll rate, $\mathrm{p}$, were found to have the best lateral matches from all three models.

All linear models used the same inputs from the B777 flight data and the corresponding outputs were compared with the measured flight data. Figures 7.4.3-7.4.6 show the evolution of the longitudinal and lateral-directional states and output variables for the true system and the six identified models. The analysis was performed using the entire flight time history for the validation process. For Figures 7.4.3-7.4.6, maneuvers were randomly selected to show a representation for all of the linear models. Table's 7.4.1 and 7.4.2 below display in percentages the Root Mean Square (RMS) error divided by the range of variation of each variable.

Table 7.4.1 Error percentage for longitudinal results

\begin{tabular}{|c|c|c|c|}
\hline Variable & no vel & id6 & pEst \\
\hline $\mathrm{a}_{\mathrm{n}}$ & 4.72 & 3.98 & 4.34 \\
\hline$\alpha$ & 13.51 & 11.80 & 11.66 \\
\hline $\mathrm{q}$ & 3.19 & 3.12 & 4.09 \\
\hline
\end{tabular}

Table 7.4.2 Error percentage for lateral-directional results

\begin{tabular}{|c|c|c|c|}
\hline Variable & no phi & id6 & pEst \\
\hline $\mathrm{a}_{\mathrm{y}}$ & 9.24 & 7.93 & 8.73 \\
\hline$\beta$ & 9.41 & 11.38 & 13.50 \\
\hline $\mathrm{p}$ & 11.08 & 7.55 & 7.48 \\
\hline $\mathrm{r}$ & 9.54 & 14.11 & 35.84 \\
\hline
\end{tabular}

Using the results from the longitudinal case (Table 7.4.1), the "id6" and "pEst" model faired to be the best representation when comparing the percentage error for each of the longitudinal variables. For the lateral case (Table 7.4.2), the best result with minimum RMS values came from the "id6" models, showing a clear problem from the yaw rate information, which was expected from the high noise content in yaw channel for Flight E. 

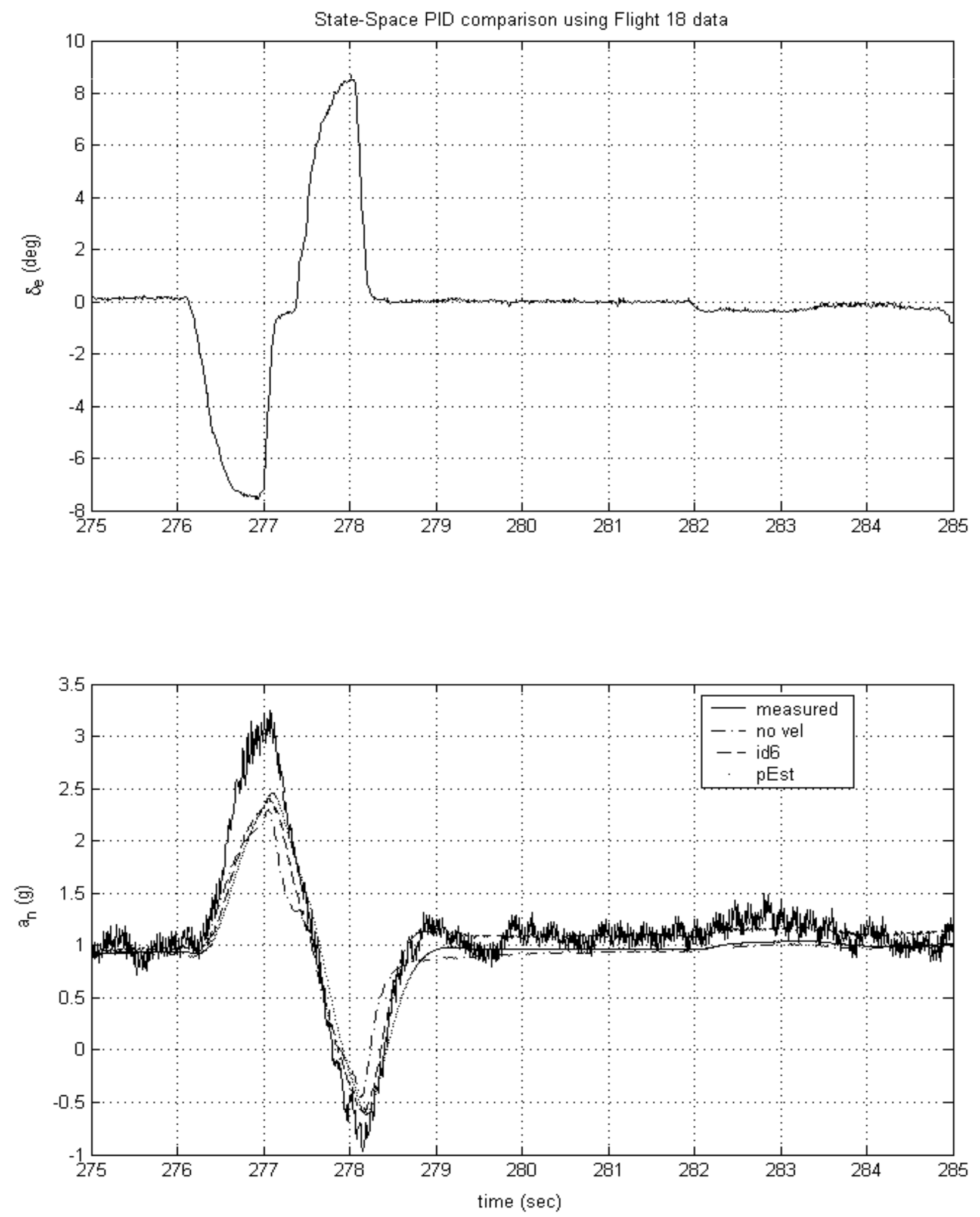

Figure 7.4.3 Comparison of state-space results for the longitudinal case for the BLS (no vel), identification toolbox (id6), and pEst with measured flight data (Flight 18) for normal acceleration (along with $\delta_{\mathrm{e}}$ input shown) 

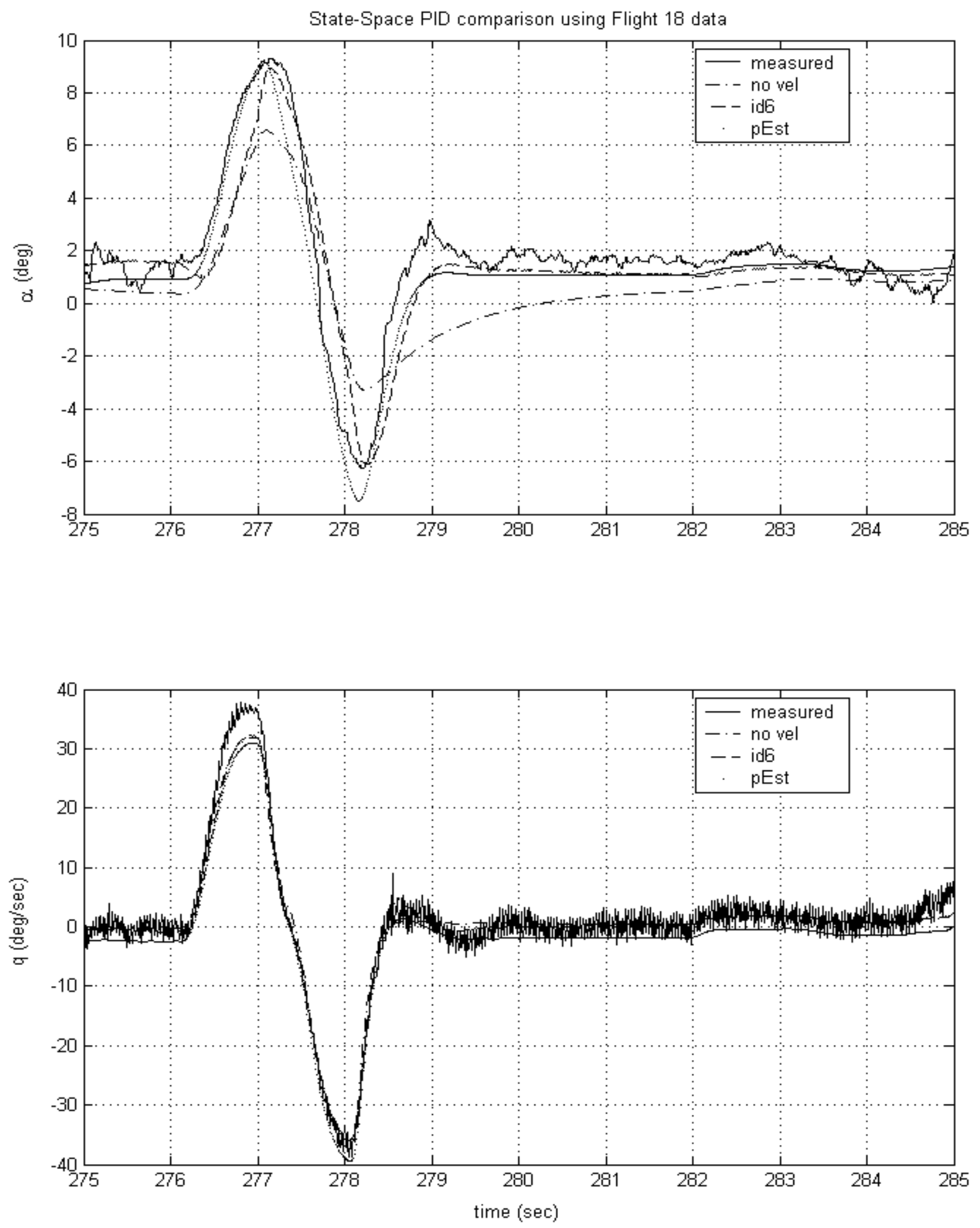

Figure 7.4.4 Comparison of state-space results for the longitudinal case for the BLS (no vel), identification toolbox (id6), and pEst with measured flight data (Flight 18) for $\alpha$ and pitch rate 

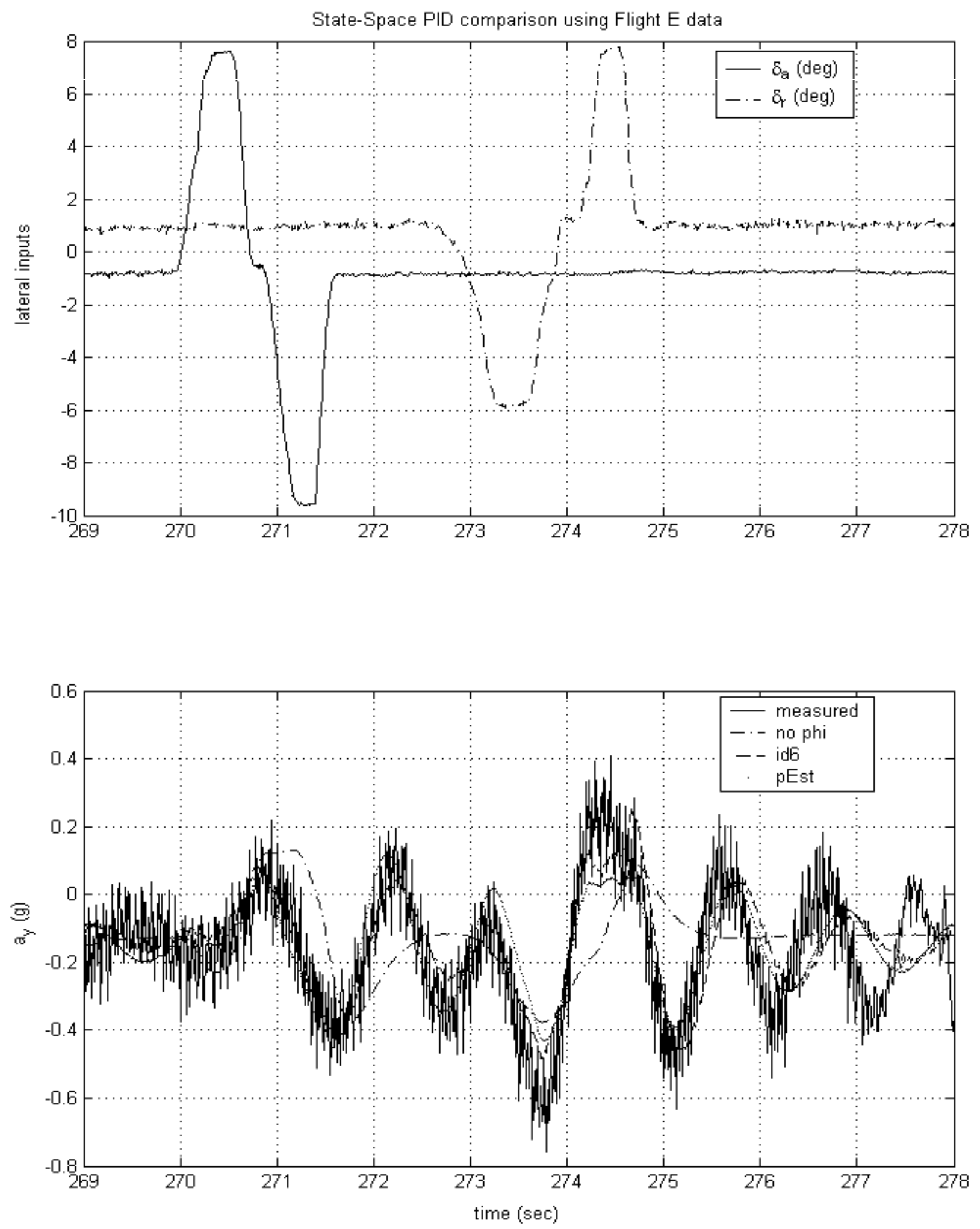

Figure 7.4.5 Comparison of state-space results for the longitudinal case for the BLS (no phil), identification toolbox (id6), and pEst with measured flight data (Flight E) for lateral acceleration (along with $\delta_{\mathrm{a}} \& \delta_{\mathrm{r}}$ inputs shown) 

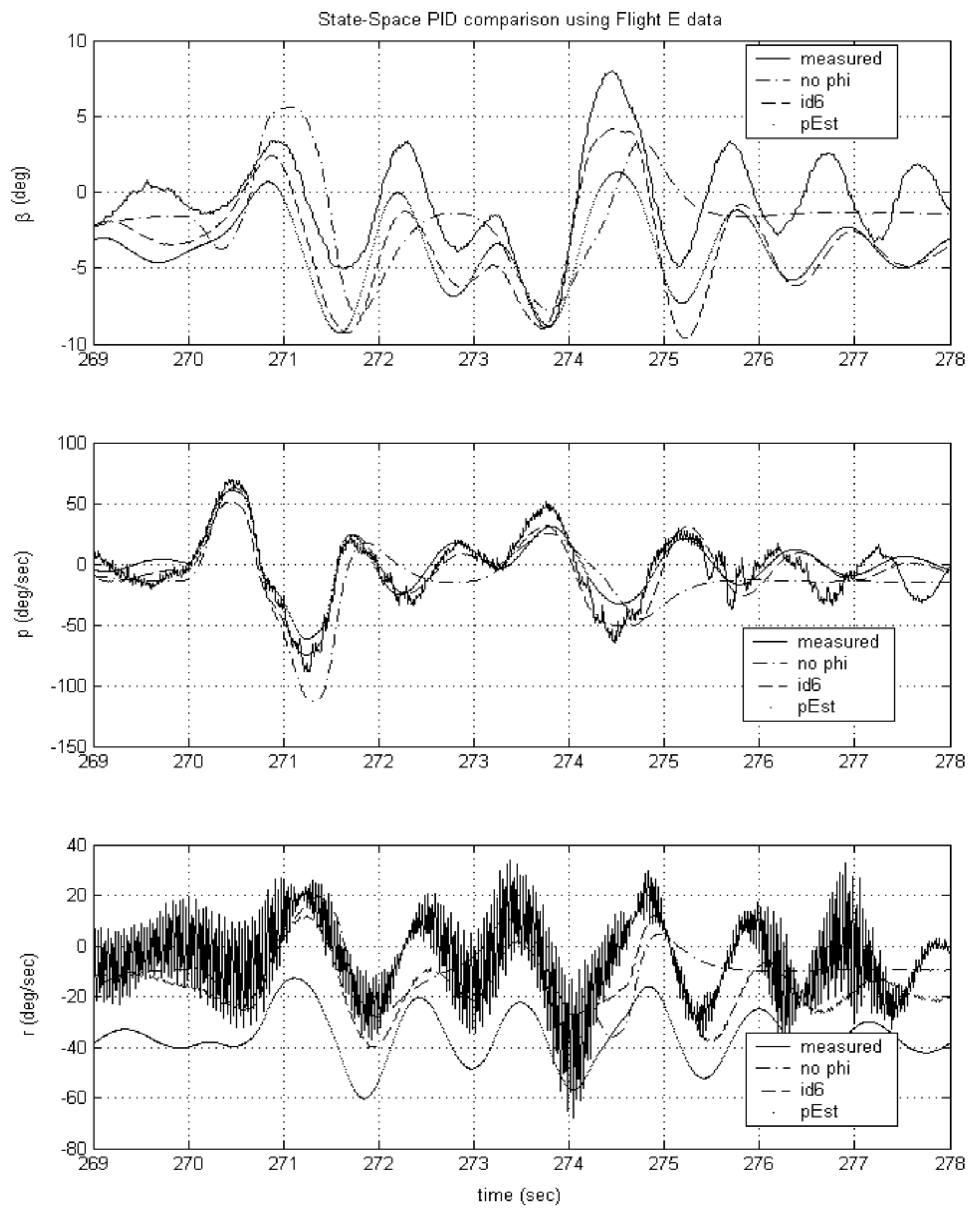

Figure 7.4.6 Comparison of state-space results for the longitudinal case for the BLS (no phi), identification toolbox (id6), and pEst with measured flight data (Flight $\mathrm{E}$ ) for $\beta$, roll, and yaw rate 


\section{Chapter 8}

\section{On-line (FTR) PID Comparison}

\subsection{Theoretical Buildup}

Aircraft PID maneuvers are typically performed off-line using previously recorded flight data. Input test maneuvers are planned out in advance and implemented within a flight test program. In recent years, several PID techniques have been proposed for on-line real-time applications to be implemented on-board aircraft during actual flight tests $^{14,15}$ which has potential in time varying control schemes. The objective of this phase was to use the ML PID results and compare results with the Fourier Transform Regression (FTR) method. Use of this method has potential for use with a real time online PID application. A brief review of the theoretic buildup will now be discussed.

The on-line PID method featured in this study was based in the frequency domain and featured a single-step technique based on Discrete Time Fourier Transform ${ }^{14,15}$ (DTFT). This PID technique was introduced in work completed by Morelli ${ }^{14,15,16}$ and Klein $^{35}$. The method has been generalized to use the output equations, in lieu of state equations to provide direct estimates of the dimensionless stability derivatives within the body axes. In fact, in its original formulation, the method performed estimates of the coefficients of the matrices within a state variable model. Following these modifications the method has been named Fourier Transform Regression (FTR), which was then tested upon existing F/A-18 HARV flight data for a previous WVU research effort ${ }^{36}$. For general description of the aircraft dynamics ${ }^{28}$ the linearized equations are given by:

$$
\begin{aligned}
& \Delta \mathrm{T}-\mathrm{m} \Delta \mathrm{a}_{\mathrm{x}}=\overline{\mathrm{q}} \mathrm{S}\left\{\mathrm{C}_{\mathrm{X}_{\alpha}} \Delta \alpha+\mathrm{C}_{\mathrm{X}_{\mathrm{q}}}\left(\frac{\overline{\mathrm{c}}}{2 \mathrm{~V}}\right) \Delta \mathrm{q}+\mathrm{C}_{\mathrm{X}_{\mathrm{u}}} \frac{\Delta \mathrm{u}}{\mathrm{V}}+\mathrm{C}_{\mathrm{X}_{\bar{\delta}}} \Delta \delta_{\mathrm{e}}\right\} \\
& \mathrm{m} \Delta \mathrm{a}_{\mathrm{z}}=\overline{\mathrm{q}} \mathrm{S}\left\{\mathrm{C}_{\mathrm{Z}_{\alpha}} \Delta \alpha+\mathrm{C}_{\mathrm{Z}_{\mathrm{q}}}\left(\frac{\overline{\mathrm{c}}}{2 \mathrm{~V}}\right) \Delta \mathrm{q}+\mathrm{C}_{\mathrm{Z}_{\mathrm{u}}} \frac{\Delta \mathrm{u}}{\mathrm{V}}+\mathrm{C}_{\mathrm{Z}_{\delta \mathrm{c}}} \Delta \delta_{\mathrm{e}}\right\} \\
& \mathrm{m} \Delta \mathrm{a}_{\mathrm{y}}=\overline{\mathrm{q}} \mathrm{S}\left\{\mathrm{C}_{\mathrm{Y}_{\beta}} \Delta \beta+\mathrm{C}_{\mathrm{Y}_{\mathrm{p}}}\left(\frac{\mathrm{b}}{2 \mathrm{~V}}\right) \Delta \mathrm{p}+\mathrm{C}_{\mathrm{Y}_{\mathrm{r}}}\left(\frac{\mathrm{r}}{2 \mathrm{~V}}\right) \Delta \mathrm{r}+\mathrm{C}_{\mathrm{Y}_{\delta \mathrm{a}}} \Delta \delta_{\mathrm{a}}+\mathrm{C}_{\mathrm{Y}_{\delta \mathrm{r}}} \Delta \delta_{\mathrm{r}}\right\} \\
& \mathrm{I}_{\mathrm{x}} \Delta \dot{\mathrm{p}}-\mathrm{I}_{\mathrm{xz}} \Delta \dot{\mathrm{r}}=\overline{\mathrm{q}} \mathrm{Sb}\left\{\mathrm{C}_{\mathrm{l}_{\beta}} \Delta \beta+\mathrm{C}_{\mathrm{l}_{\mathrm{p}}}\left(\frac{\mathrm{b}}{2 \mathrm{~V}}\right) \Delta \mathrm{p}+\mathrm{C}_{\mathrm{l}_{\mathrm{r}}}\left(\frac{\mathrm{b}}{2 \mathrm{~V}}\right) \Delta \mathrm{r}+\mathrm{C}_{\mathrm{l}_{\delta a}} \Delta \delta_{\mathrm{a}}+\mathrm{C}_{\mathrm{l}_{\delta \mathrm{r}}} \Delta \delta_{\mathrm{r}}\right\} \\
& \mathrm{I}_{\mathrm{z}} \Delta \dot{\mathrm{r}}-\mathrm{I}_{\mathrm{xz}} \Delta \dot{\mathrm{p}}=\overline{\mathrm{q}} \mathrm{Sb}\left\{\mathrm{C}_{\mathrm{n}_{\beta}} \Delta \beta+\mathrm{C}_{\mathrm{n}_{\mathrm{p}}}\left(\frac{\mathrm{b}}{2 \mathrm{~V}}\right) \Delta \mathrm{p}+\mathrm{C}_{\mathrm{n}_{\mathrm{r}}}\left(\frac{\mathrm{b}}{2 \mathrm{~V}}\right) \Delta \mathrm{r}+\mathrm{C}_{\mathrm{n}_{\delta_{a}}} \Delta \delta_{\mathrm{a}}+\mathrm{C}_{\mathrm{n}_{\delta_{\mathrm{rr}}}} \Delta \delta_{\mathrm{r}}\right\} \\
& \mathrm{I}_{\mathrm{y}} \Delta \dot{\mathrm{q}}=\overline{\mathrm{q}} \mathrm{S} \overline{\mathrm{c}}\left\{\mathrm{C}_{\mathrm{m}_{\alpha}} \Delta \alpha+\mathrm{C}_{\mathrm{m}_{\mathrm{q}}}\left(\frac{\overline{\mathrm{c}}}{2 \mathrm{~V}}\right) \Delta \mathrm{q}+\mathrm{C}_{\mathrm{m}_{\mathrm{u}}} \frac{\Delta \mathrm{u}}{\mathrm{V}}+\mathrm{C}_{\mathrm{m}_{\delta e}} \Delta \delta_{\mathrm{e}}\right\}
\end{aligned}
$$


A general form for each of the equations above can be given by:

$$
E \dot{z}(t)+F z(t)=x(t)^{T} \Theta
$$

where E,F are known constant vectors and $\Theta$ is an unknown constant vector to be estimated. For example, for the pitching moment equation we would have:

$$
\begin{gathered}
\mathrm{x}=\left[\Delta \alpha, \Delta \mathrm{q}, \Delta \delta_{\mathrm{e}}\right]^{\mathrm{T}} \\
E z=I_{y} \Delta q \\
\mathrm{Fz}=0
\end{gathered}
$$

Sampling and applying DTFT to the input and motion variables at time $t=i \Delta t$ we have:

$$
j \omega E \tilde{z}(\omega)+F \tilde{z}(\omega)=\tilde{x}(\omega)^{T} \Theta
$$

where

$$
\tilde{x}(\omega)=\sum_{0}^{N-1} x(i \Delta t) e^{-j \omega \Delta t}, \quad \tilde{z}(\omega)=\sum_{0}^{N-1} z(i \Delta t) e^{-j \omega i \Delta t}
$$

As in the general LS regression method, the measurements of the vectors $x$ and $z$ can be used to set up a cost function having the coefficients of $\Theta$ as an argument. In particular, one can set $m$ algebraic equations over a set of frequency points $\left[\omega_{1}, \omega_{2}, \cdots, \omega_{m}\right]$ :

$$
\left[\begin{array}{c}
j \omega_{1} E \tilde{y}\left(\omega_{1}\right)+F \tilde{z}\left(\omega_{1}\right) \\
j \omega_{2} E \tilde{y}\left(\omega_{2}\right)+F \tilde{z}\left(\omega_{2}\right) \\
\cdots \\
\cdots \\
j \omega_{m} E \tilde{y}\left(\omega_{m}\right)+F \tilde{z}\left(\omega_{m}\right)
\end{array}\right]=\left[\begin{array}{c}
\tilde{x}^{T}\left(\omega_{1}\right) \\
\tilde{x}^{T}\left(\omega_{2}\right) \\
\cdots \\
\cdots \\
\tilde{x}^{T}\left(\omega_{m}\right)
\end{array}\right] \Theta
$$

Introducing a complex error vector $\varepsilon$, which accounts for noise and non-linearities, the above equations can be rewritten into the general form $Y=X \Theta+\mathcal{E}$ with conventional definitions for $Y, X$, and $\Theta$. Thus the problem can be formulated as a LS regression problem with the following complex cost function:

$$
J=\frac{1}{2}(Y-X \Theta)^{*}(Y-X \Theta)
$$

The solution is given by:

$$
\hat{\Theta}=\left[\operatorname{Re}\left(X^{*} X\right)\right]^{-1} \operatorname{Re}\left(X^{*} Y\right)
$$


where ${ }^{*}$ indicates a complex conjugate transpose. The cost function is made of a summation over $m$ frequencies of interest. In addition, the covariance matrix of the estimates of $\hat{\Theta}$ is computed as

$$
\operatorname{cov}(\hat{\Theta})=E\left\{(\hat{\Theta}-\Theta)(\hat{\Theta}-\Theta)^{*}\right\}=\sigma^{2}(\hat{\Theta}) \cdot\left[\operatorname{Re}\left(X^{*} X\right)\right]^{-1}
$$

where $\sigma^{2}(\hat{\Theta})$ is the equation error variance and can be estimated on-line using

$$
\hat{\sigma}^{2}(\hat{\Theta})=\frac{1}{(m-p)}\left[(Y-X \hat{\Theta})^{*}(Y-X \hat{\Theta})\right]
$$

where $p$ is the number of parameters to be estimated and $m$ is the number of frequency points. The standard deviation of the estimation error for the $l$-th unknown of the $p$ parameters in $\hat{\Theta}$ can be evaluated as the square root of the $(l, l)$ coefficient (maindiagonal coefficient) of the covariance matrix. This standard deviation allows for an online assessment of the accuracy of the parameter estimates. For a given frequency, $\omega_{n}$, the DTFT at the $i$-th time step was related to the DTFT at the (i-1)-th time step as follows:

$$
\tilde{x}_{i}\left(\omega_{n}\right)=\tilde{x}_{i-1}\left(\omega_{n}\right)+x_{i} e^{-j \omega_{n} i \Delta t}
$$

Showing that on-line computation of $\tilde{x}_{i}\left(\omega_{n}\right)$ requires low computational effort. In addition, the scheme requires only a fixed memory space for $\tilde{x}_{i}(\omega)$ even if it is updated at every step. An important characteristic of the DTFT approach was that it allows for the retaining of PID results from previous time steps and, at the same time, can provide the necessary flexibility to follow changes in the system dynamics. 


\section{$\underline{\text { FTR Simulink simulation }}$}

Inputs for the longitudinal FTR included $\alpha$, pitch rate and elevator deflection. For lateral-directional FTR, parameters included $\beta$, roll rate, yaw rate, and deflections of the aileron and rudder control surfaces. Figure 8.1.1 shows the Simulink FTR scheme used:

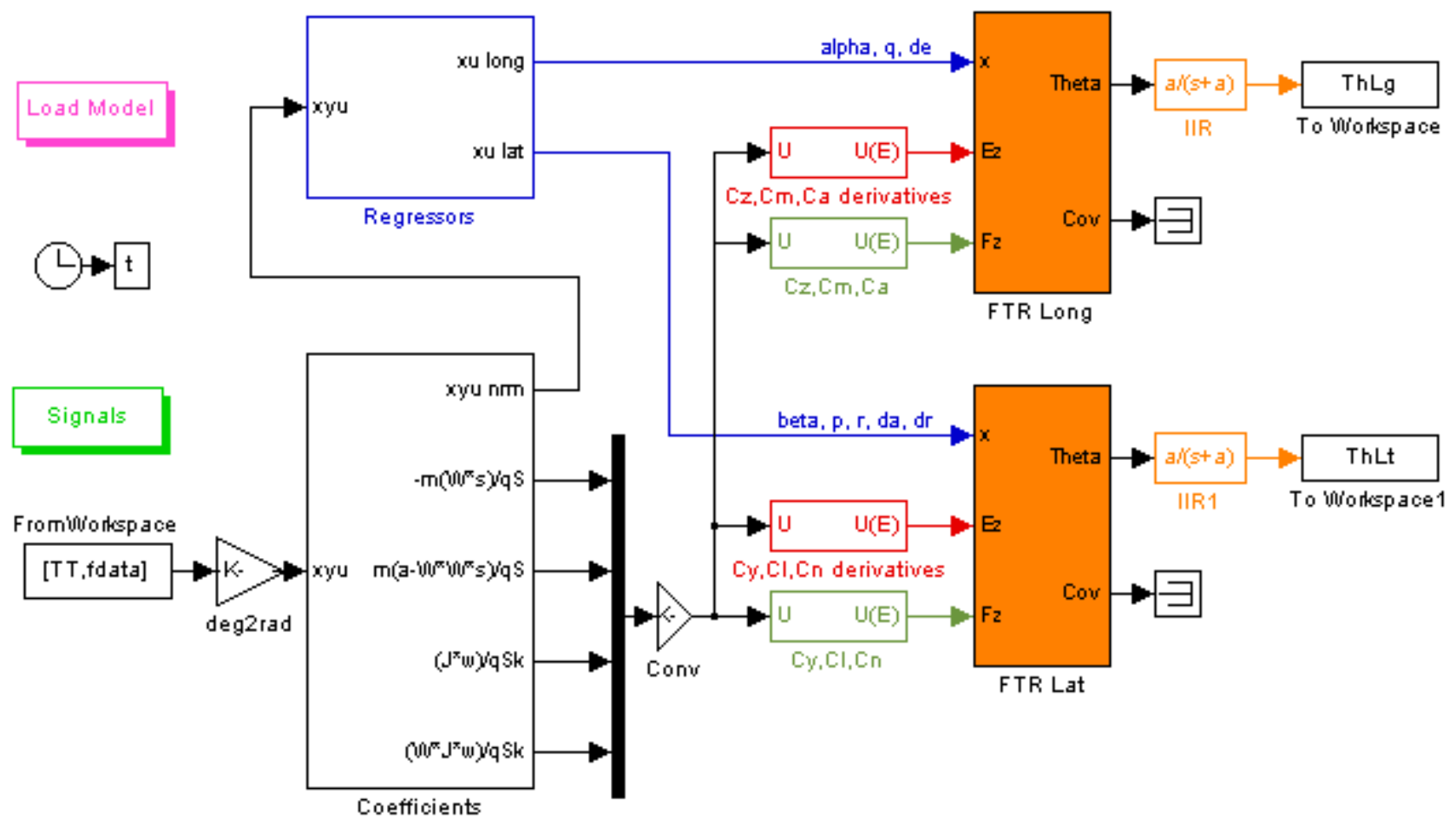

Figure 8.1.1 Simulink FTR scheme for longitudinal and lateral-directional dynamics

As shown in the scheme above, the entire set of flight data was provided from the Matlab workspace. The flight information was then passed to the "coefficients" box that compute the aircraft forces and moments using angular rates, linear accelerations and mass/geometric aircraft information. The aircraft flight information such as flow angles, angular rates and control surfaces were then fed into the FTR blocks, along with computed forces and moments. Output information from the FTR blocks was then stored in the Matlab workspace to be used for comparison purposes. After completion of the simulation, a complete time history of the aircraft estimates was obtained. Estimates for a specific maneuver could then be selected and plotted against the coefficient values obtained from phase two. 


\subsection{Longitudinal and Lateral-Directional Results}

Figures 8.2.1-8.1.20 show results from the FTR scheme plotted with the associated phase two values for a particular flight maneuver.. For this analysis, complete time histories with the Simulink scheme were evaluated for Flights C, D, and E. The breakdown of maneuvers shown are representative of:

- one elevator input;

- two aileron inputs;

- two rudder inputs;

- two aileron and rudder combination inputs.

As a note, Flight D had included a useable elevator input maneuver for use with this phase because all of the angular rate information was available. For each maneuver shown, a corresponding measured time history was provided.

For the longitudinal maneuver, Figure 8.2.1 shows the selected measured time histories for the single elevator input. Figure 8.2.2 shows a comparison of the longitudinal coefficients $\mathrm{C}_{\mathrm{Z}}, \mathrm{C}_{\mathrm{m}}$, and $\mathrm{C}_{\mathrm{A}}$ with the pEst results. The dash line represents the FTR value and the dotted line represents the pEst constant obtained for that specific test maneuver. Overall the pitching moment coefficients were in good agreement with the pEst value computed for the specific maneuver.

For the lateral cases, three sets of input maneuvers were shown for comparison; two ailerons, two rudders, and two combination aileron and rudder doublets. Again, each FTR estimate was plotted with the associated pEst constant value. Figures 8.2.3-8.2.8 show the two $\delta_{\mathrm{a}}$ input maneuvers. In Flight D, maneuver 5, the input deflection was enough excitation to accurately obtain matches for $\mathrm{C}_{\mathrm{l}_{\beta}}$ and $\mathrm{C}_{\mathrm{n}}$, with close approximations for $\mathrm{C}_{\delta_{\mathrm{a}}}, \mathrm{C}_{\mathrm{n}_{\mathrm{r}}}$, and $\mathrm{C}_{\mathrm{n}_{\mathrm{p}}}$. For Flight $\mathrm{E}$, maneuver 10, the magnitude of the aileron deflection was smaller and apparently was able to achieve the same trends as compared to the first aileron doublet (Flight D, maneuver 5). However, the excitation was not great enough to provide exact matches for the sideslip derivatives.

Figures 8.2.9-8.2.14 describe the two individual $\delta_{\mathrm{r}}$ inputs. For Flight D, maneuver 3, and Flight E, maneuver 9 shown, the FTR provided a poor match when compared with the pEst estimates. The assumption is the aircraft excitation was not 
enough for the FTR to evaluate the aircraft dynamics. As seen within the original $\mathrm{pEst}$ estimation results, selected $\delta_{\mathrm{r}}$ inputs in Flight $\mathrm{E}$ were found to have very large CRB values, which tended to suggest a poor modeling of the aircraft dynamics. However, as with the phoase two response matches for the selected $\delta_{\mathrm{r}}$ maneuvers, a problem was not indicated until plotted against the rest of the lateral estimates.

Figures 8.2.15-8.2.20 show examples of the combination $\delta_{\mathrm{a}}$ and $\delta_{\mathrm{r}}$ lateral input maneuvers, specifically, from Flight $\mathrm{C}$ maneuver numbers 3 and 6 shown. The combination input maneuvers provided the best matches between the FTR output and the pEst values, which seems to be due to the increased excitation of lateral information. Reasonable matches were found for most of the rolling and yawing moment derivatives, specifically for $\mathrm{C}_{\mathrm{l}_{\beta}}, \mathrm{C}_{\mathrm{l}_{\mathrm{r}}}, \mathrm{C}_{\mathrm{n}_{\beta}}, \mathrm{C}_{\mathrm{l}_{\mathrm{a}}}$, and $\mathrm{C}_{\mathrm{n}_{\delta_{\mathrm{r}}}}$ had close matches with the off-line results. 
$\delta_{\mathrm{e}}$ input (Flight D - Man 4)
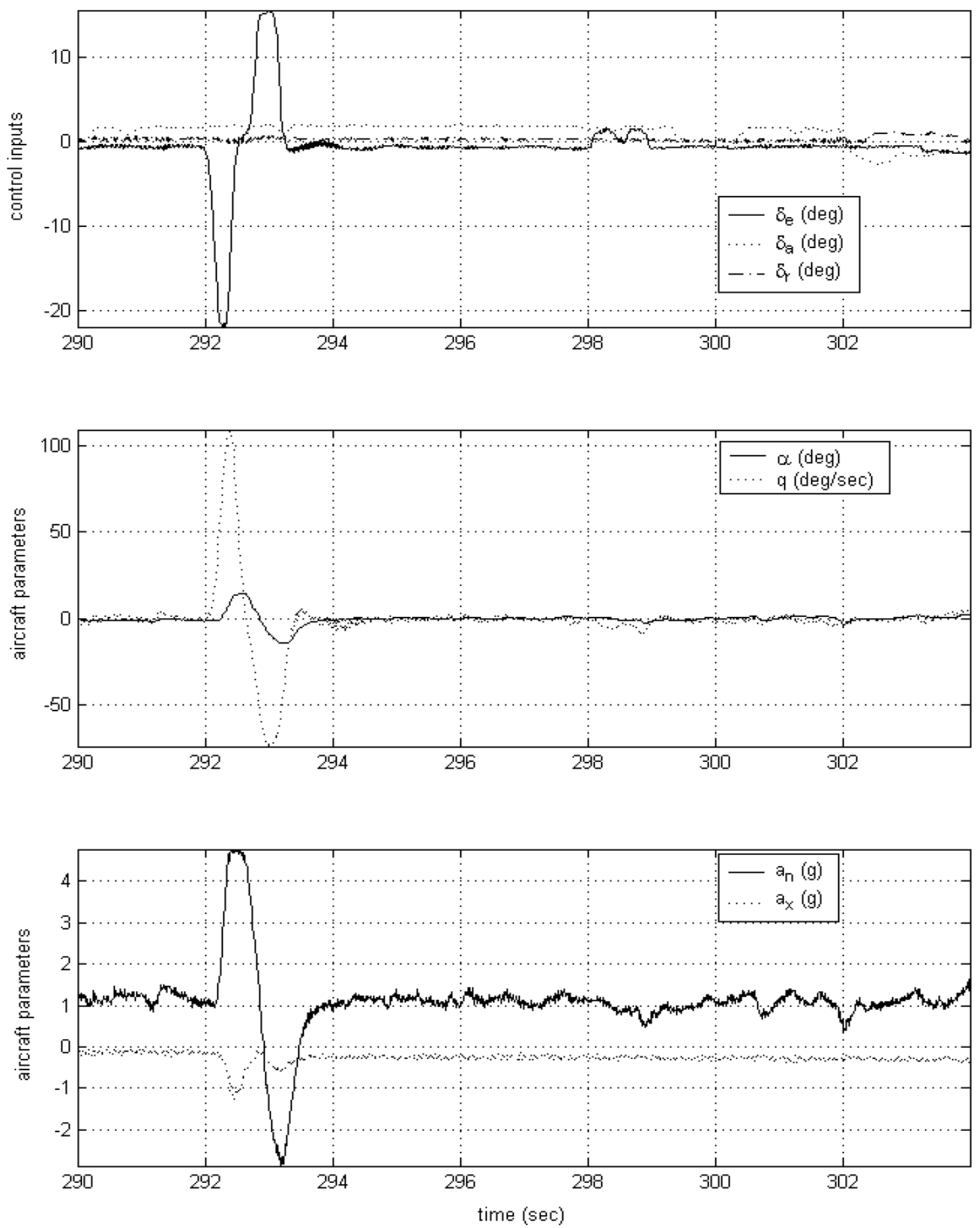

Figure 8.2.1 Measured time histories used for FTR / $p$ Est comparison for Flight D, Maneuver $4\left(\delta_{\mathrm{e}}\right.$ input $)$ 
$\delta_{\mathrm{e}}$ (Flight D - Man 4) - FTR(dash), pEst(dotted)
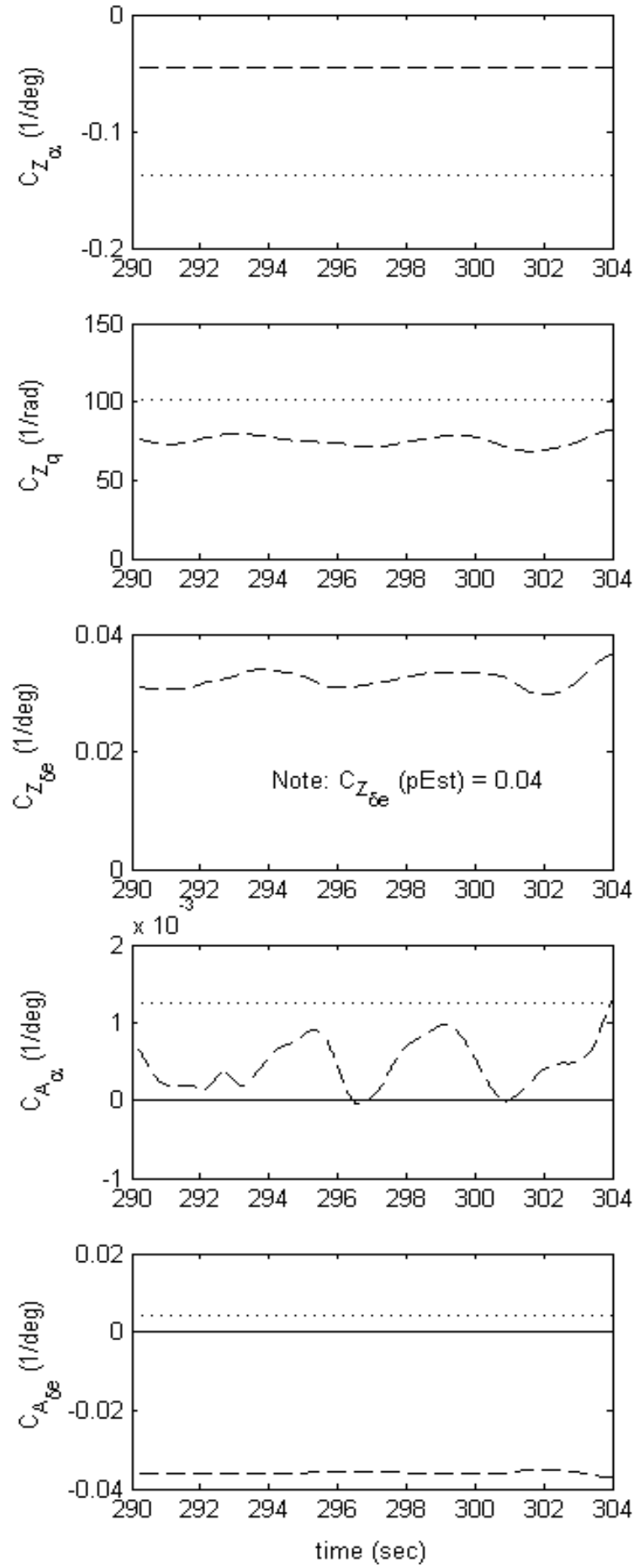

$\delta_{\mathrm{e}}$ (Flight D - Man 4) - FTR(dash), pEst(dotted)
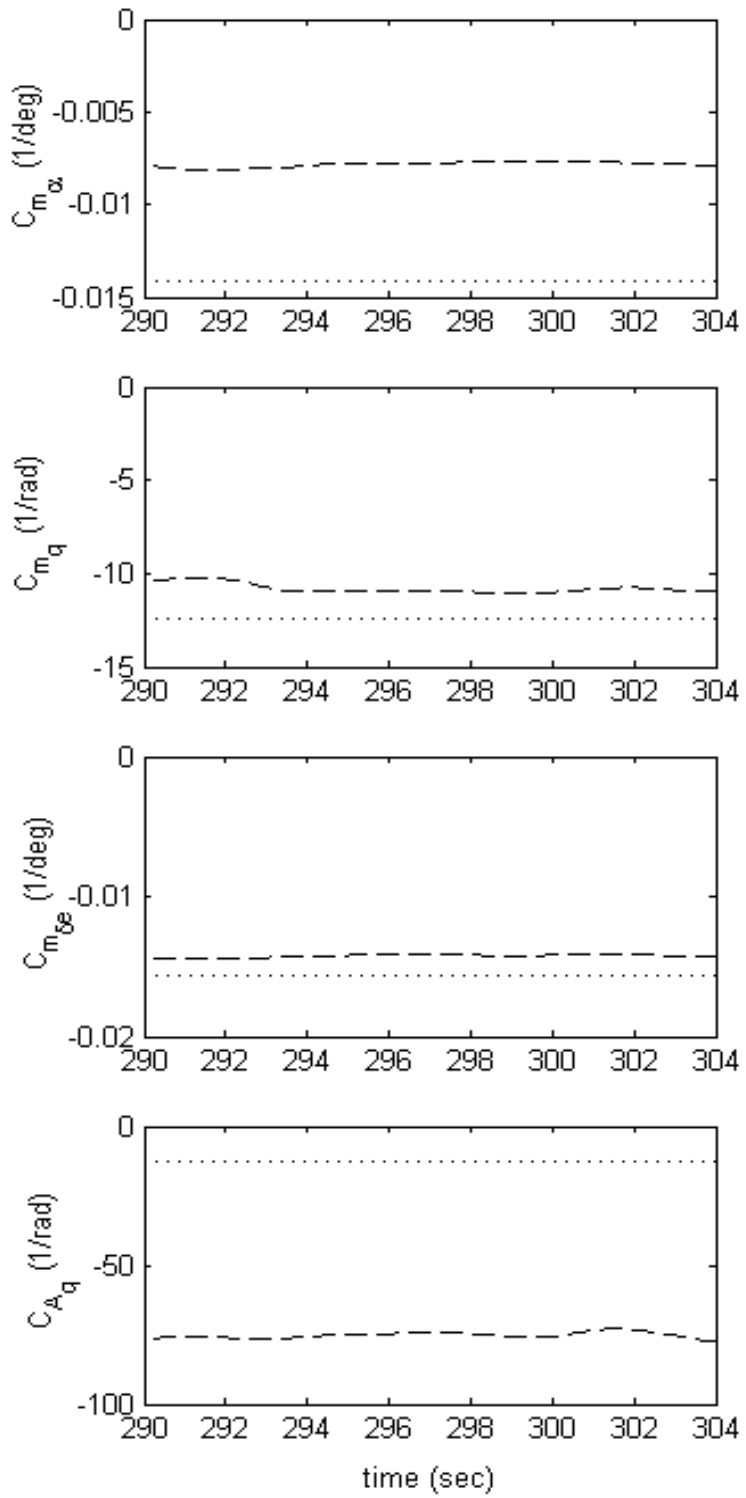

Figure 8.2.2 Comparison of FTR and pEst longitudinal coefficients $C_{Z}, C_{m}$, and $\mathrm{C}_{\mathrm{A}}$ for Flight D, Maneuver 4 ( $\delta_{\mathrm{e}}$ input) 
$\delta_{\mathrm{a}}$ input (Flight D - Man 5)
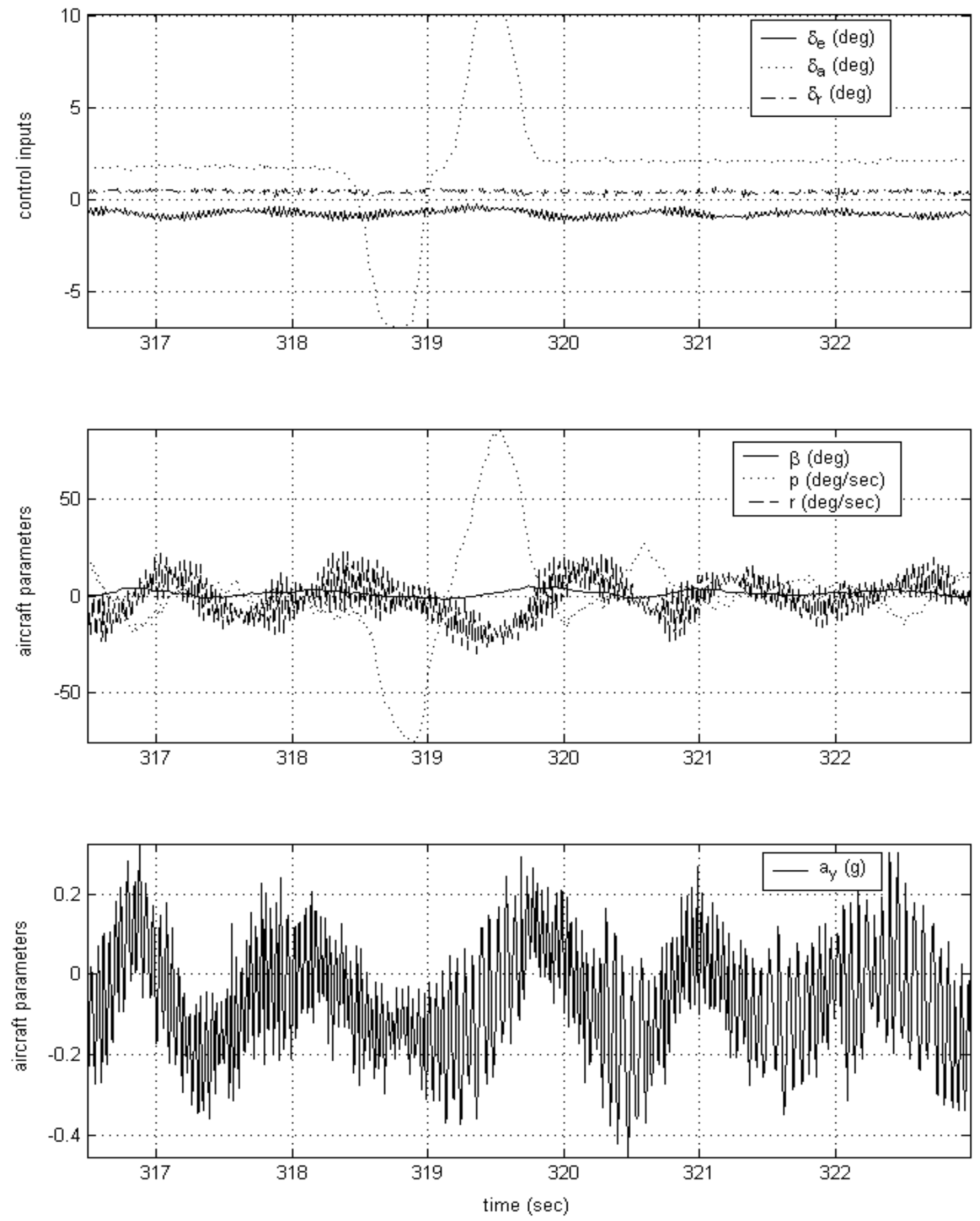

Figure 8.2.3 Measured time histories used for FTR / pEst comparison for Flight D, Maneuver 5 ( $\delta_{\mathrm{a}}$ input $)$ 
$\delta_{\mathrm{a}}$ (Flight D - Man 5) - FTR(dash), pEst(dotted)
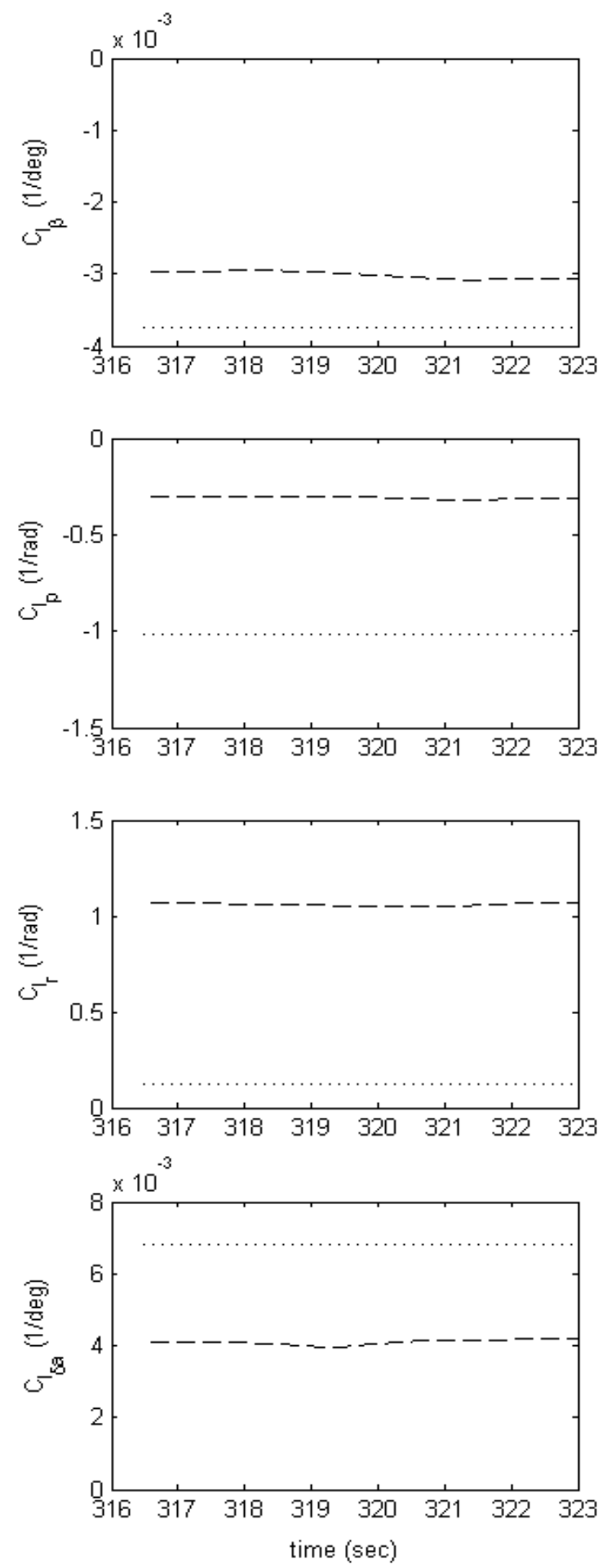

$\delta_{\mathrm{a}}$ (Flight D - Man 5) - FTR(idash), pEst(dotted)
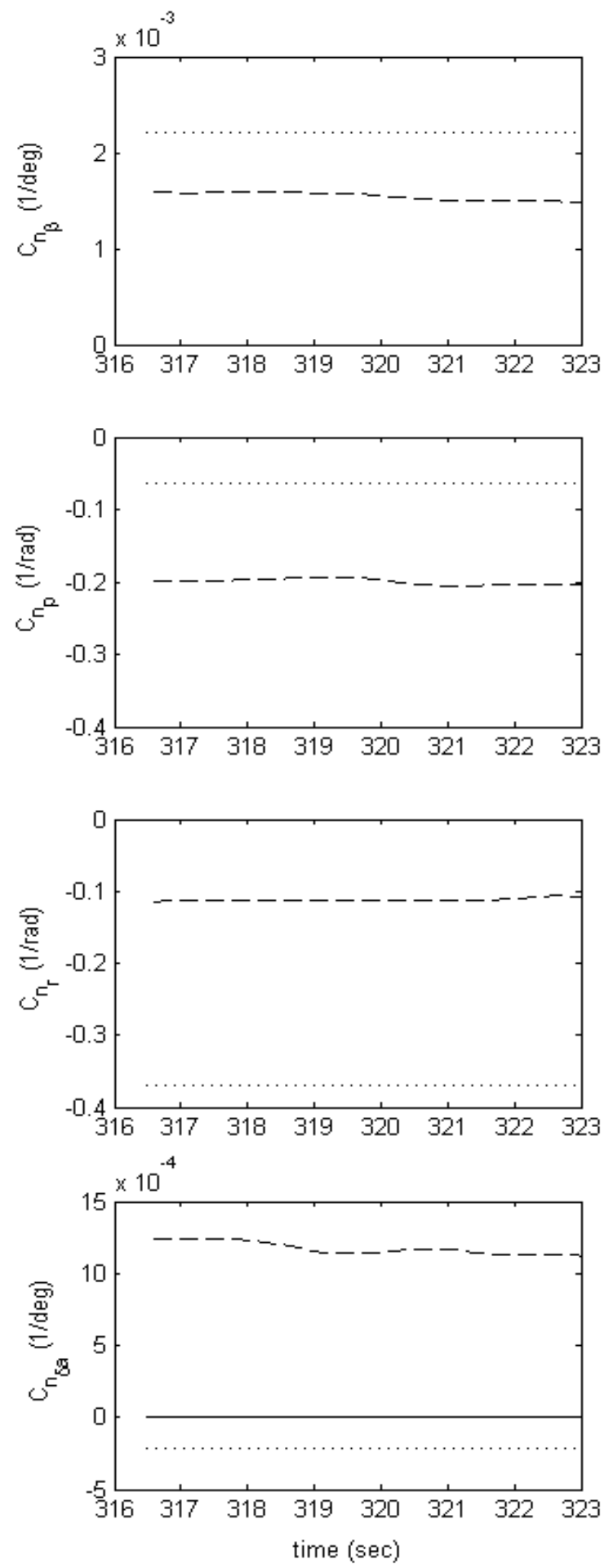

Figure 8.2.4 Comparison of lateral-directional coefficients $C_{1}$ and $C_{n}$ for Flight $\mathrm{D}$, Maneuver 5 ( $\delta_{\mathrm{a}}$ input $)$ 
$\delta_{a}$ (Flight D - Man 5) - FTR(dash), pEst(dotted)
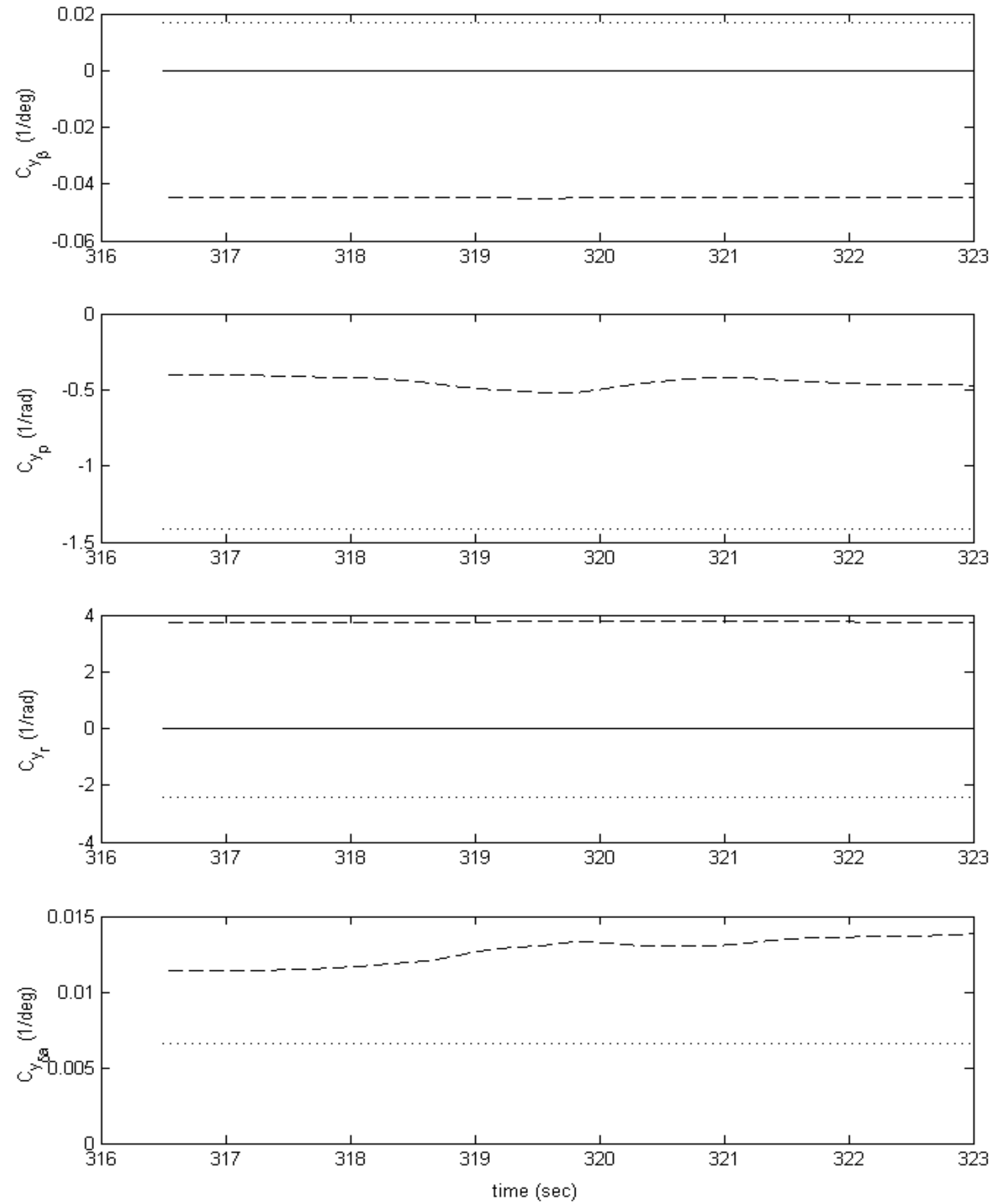

Figure 8.2.5 Comparison of lateral-directional coefficients $C_{y}$ for Flight $D$, Maneuver 5 ( $\delta_{\mathrm{a}}$ input $)$ 
$\delta_{a}$ input (Flight E - Man 10)
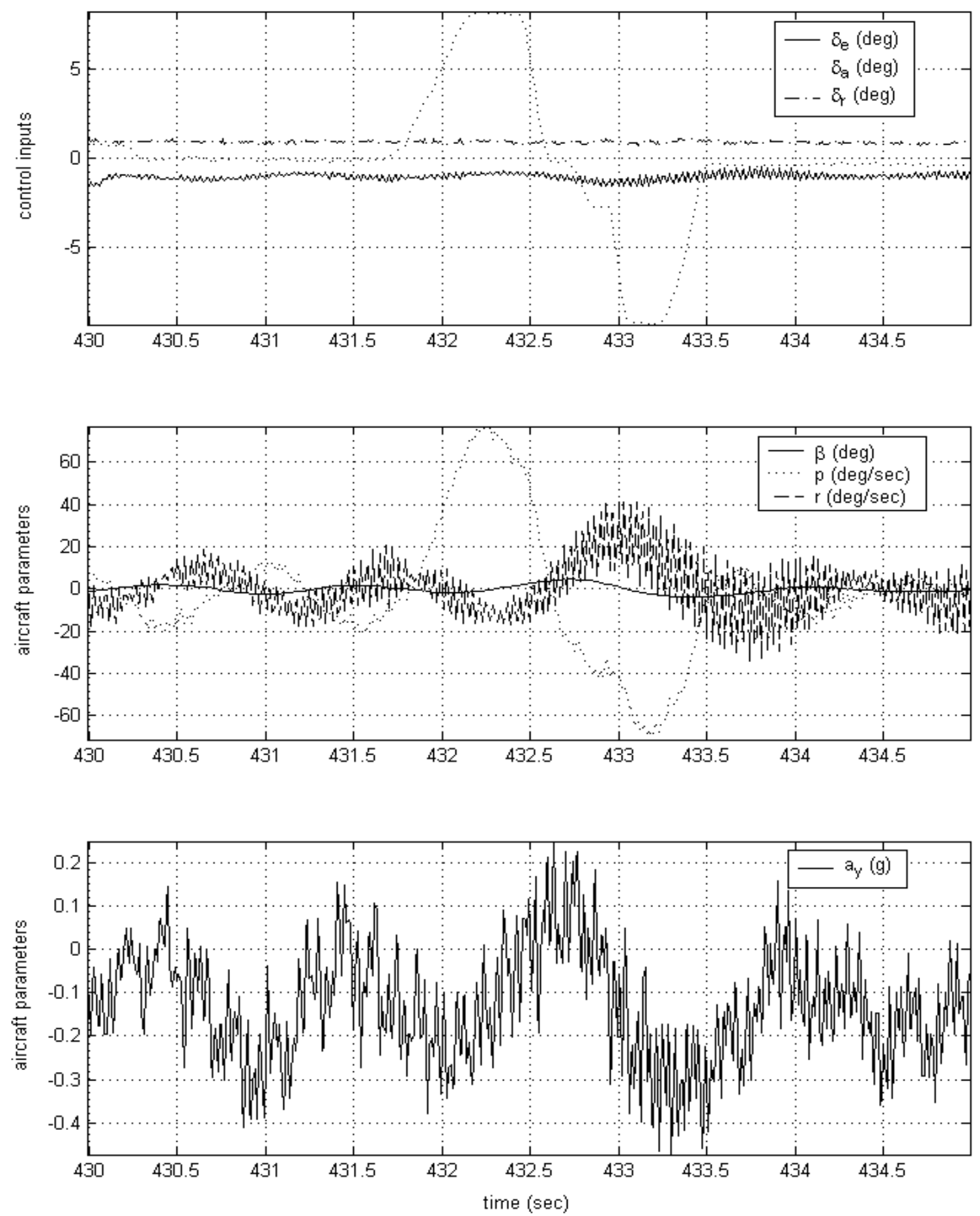

Figure 8.2.6 Measured time histories used for FTR / pEst comparison for Flight E, Maneuver $10\left(\delta_{\mathrm{a}}\right.$ input $)$ 

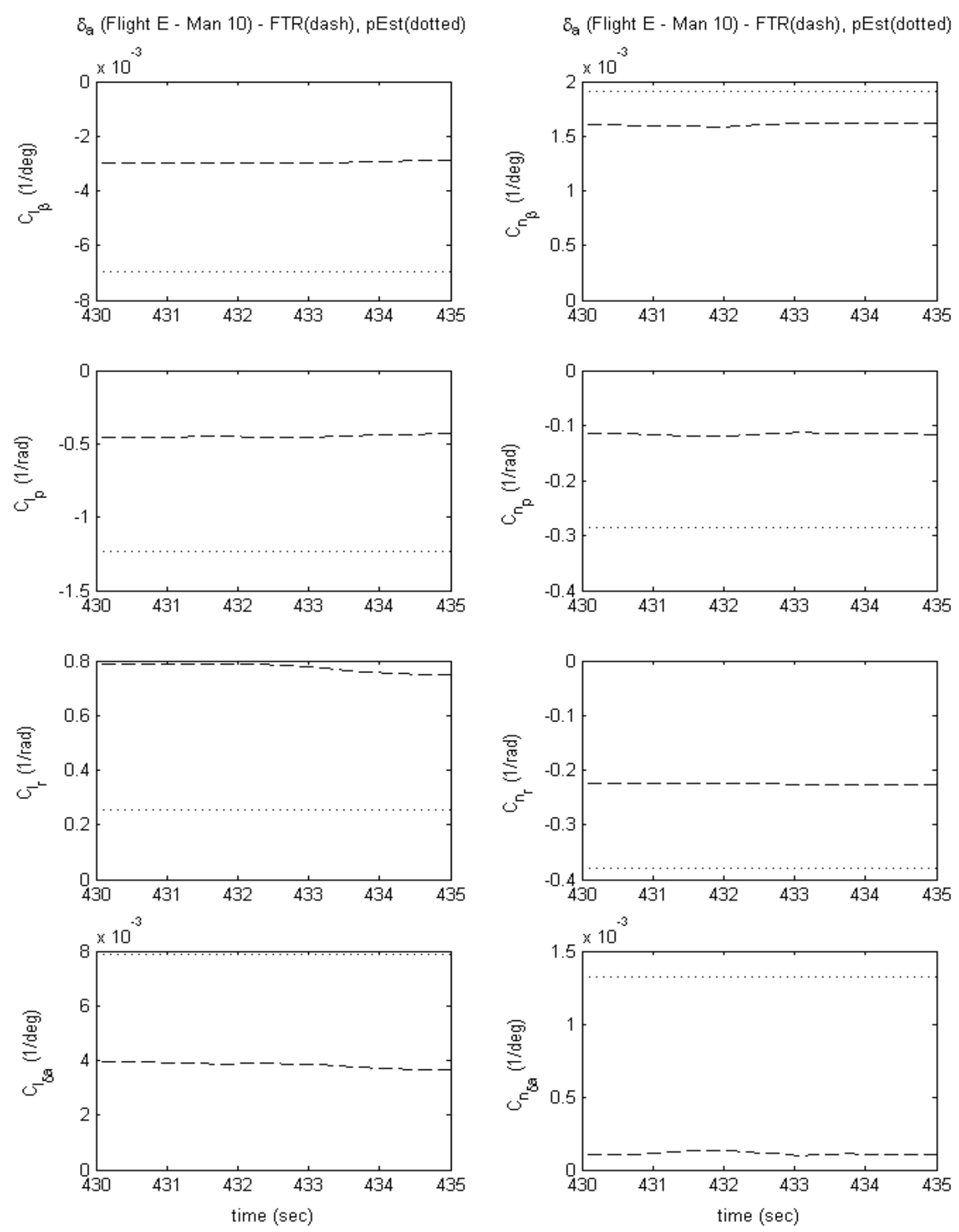

Figure 8.2.7 Comparison of lateral-directional coefficients $C_{1}$ and $C_{n}$ for Flight E, Maneuver 10 ( $\delta_{\mathrm{a}}$ input $)$ 
$\delta_{\mathrm{a}}$ (Flight E - Man 10) - FTR(dash), pEst(dotted)
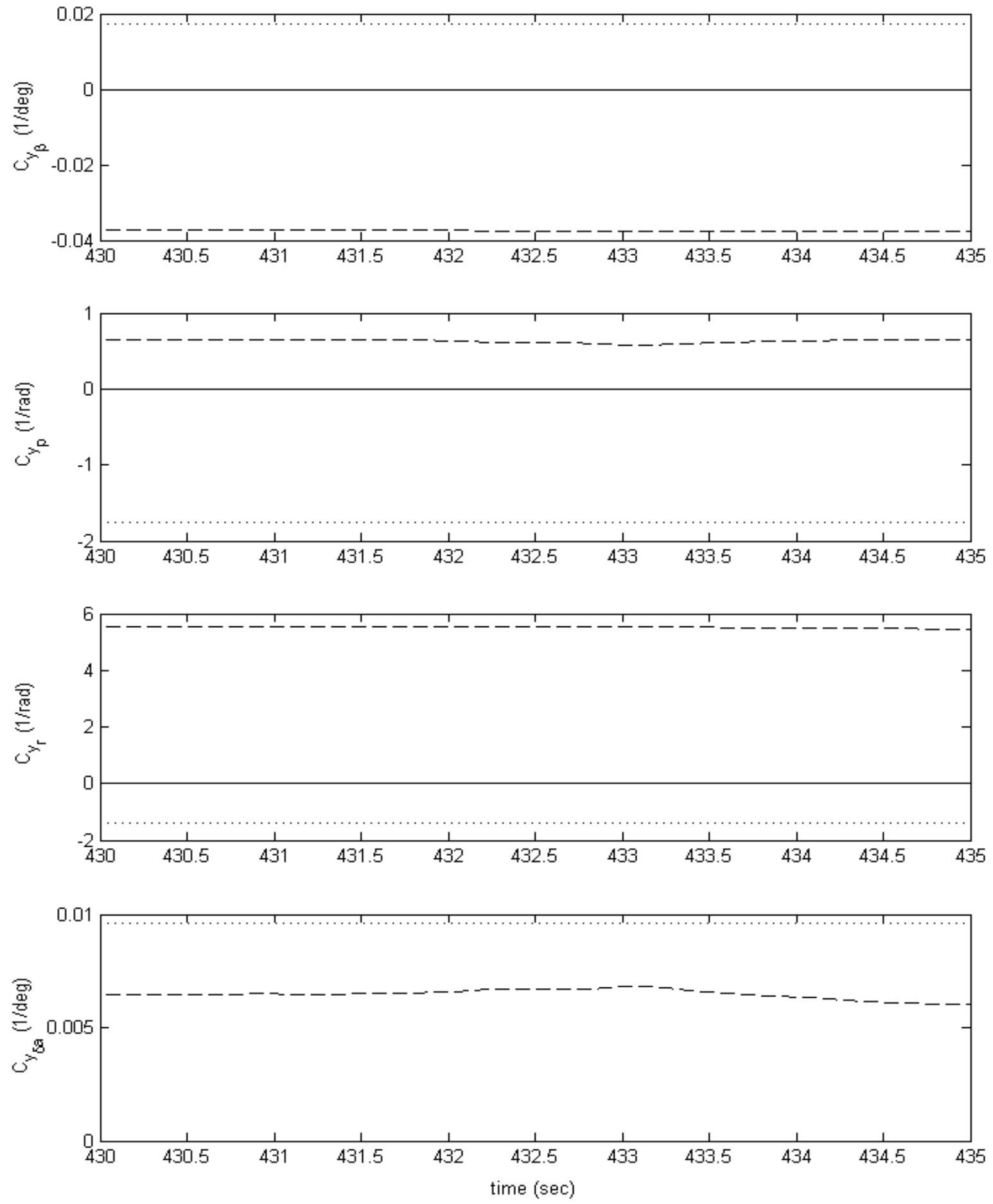

Figure 8.2.8 Comparison of lateral-directional coefficients $C_{y}$ for Flight $E$, Maneuver $10\left(\delta_{\mathrm{a}}\right.$ input $)$ 
§, input (Flight D - Man 3)
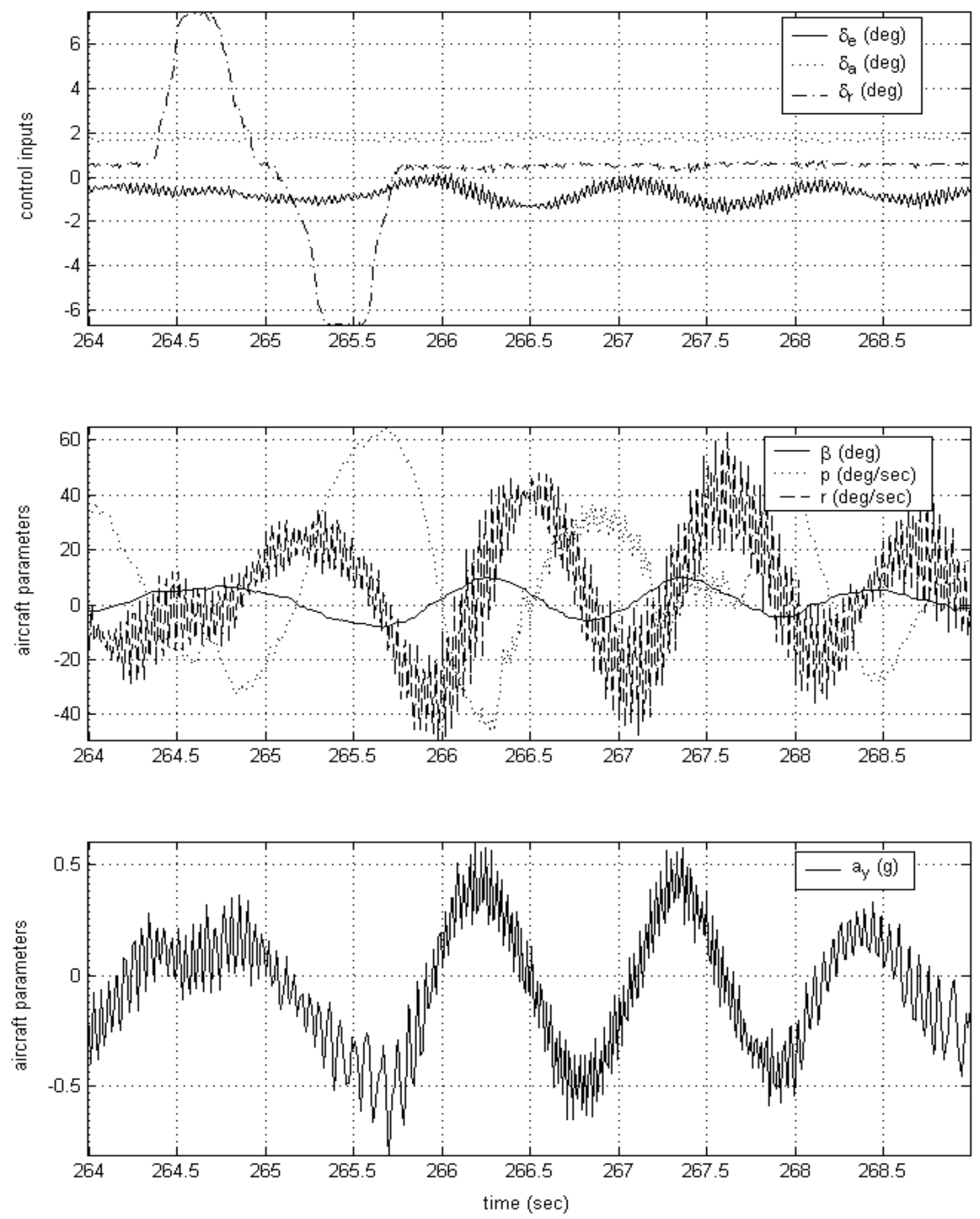

Figure 8.2.9 Measured time histories used for FTR / pEst comparison for Flight D, Maneuver $3\left(\delta_{\mathrm{r}}\right.$ input $)$ 


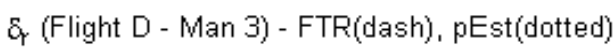
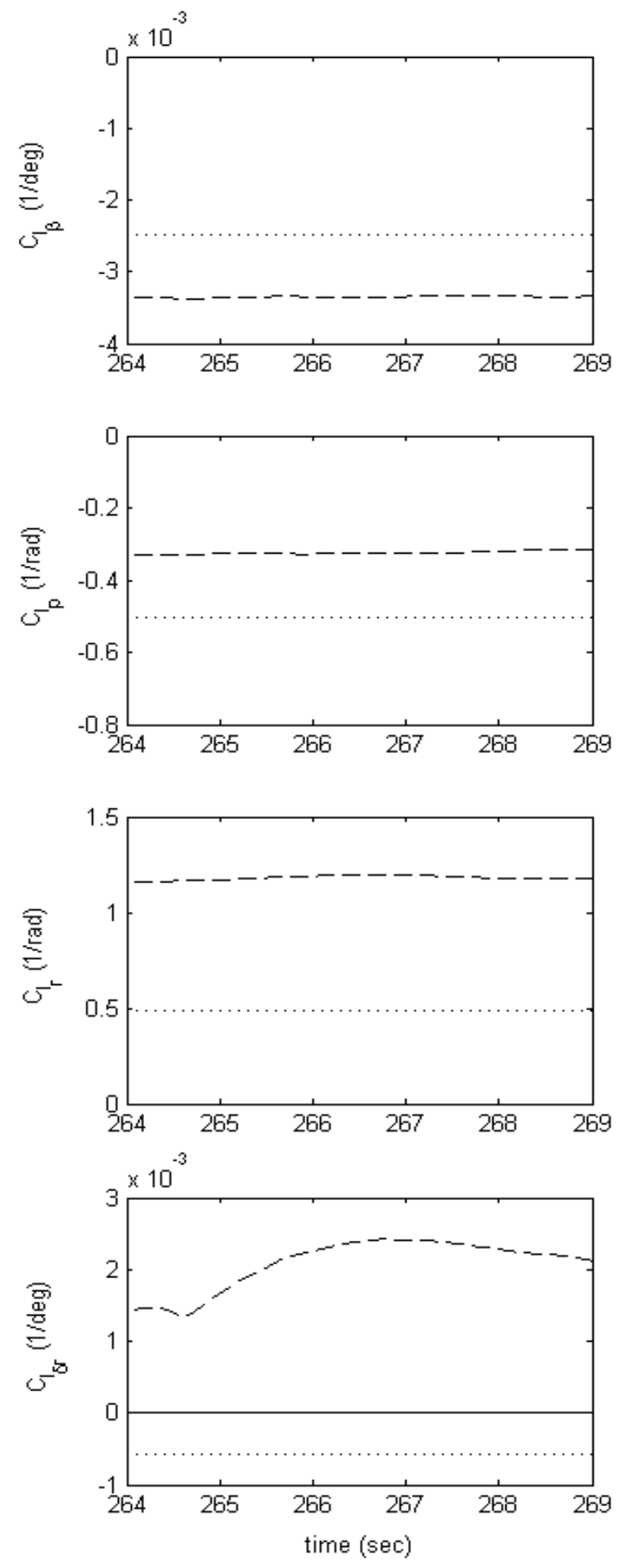

$\delta$ (Flight D - Man 3) - FTR(dash), pEst(dotted)
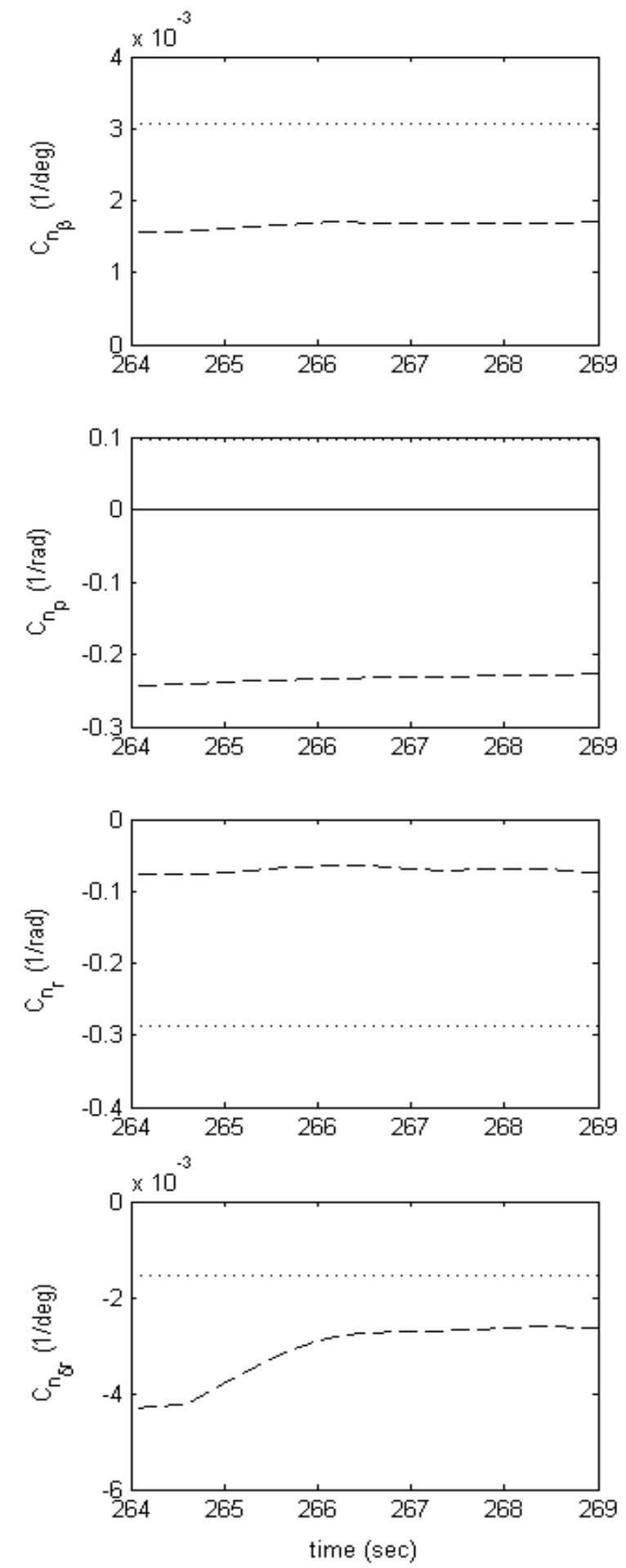

Figure 8.2.10 Comparison of lateral-directional coefficients $C_{1}$ and $C_{n}$ for Flight D, Maneuver 3 ( $\delta_{\mathrm{r}}$ input $)$ 
¿. (Flight D - Man 3) - FTR(dash), pEst(dotted)
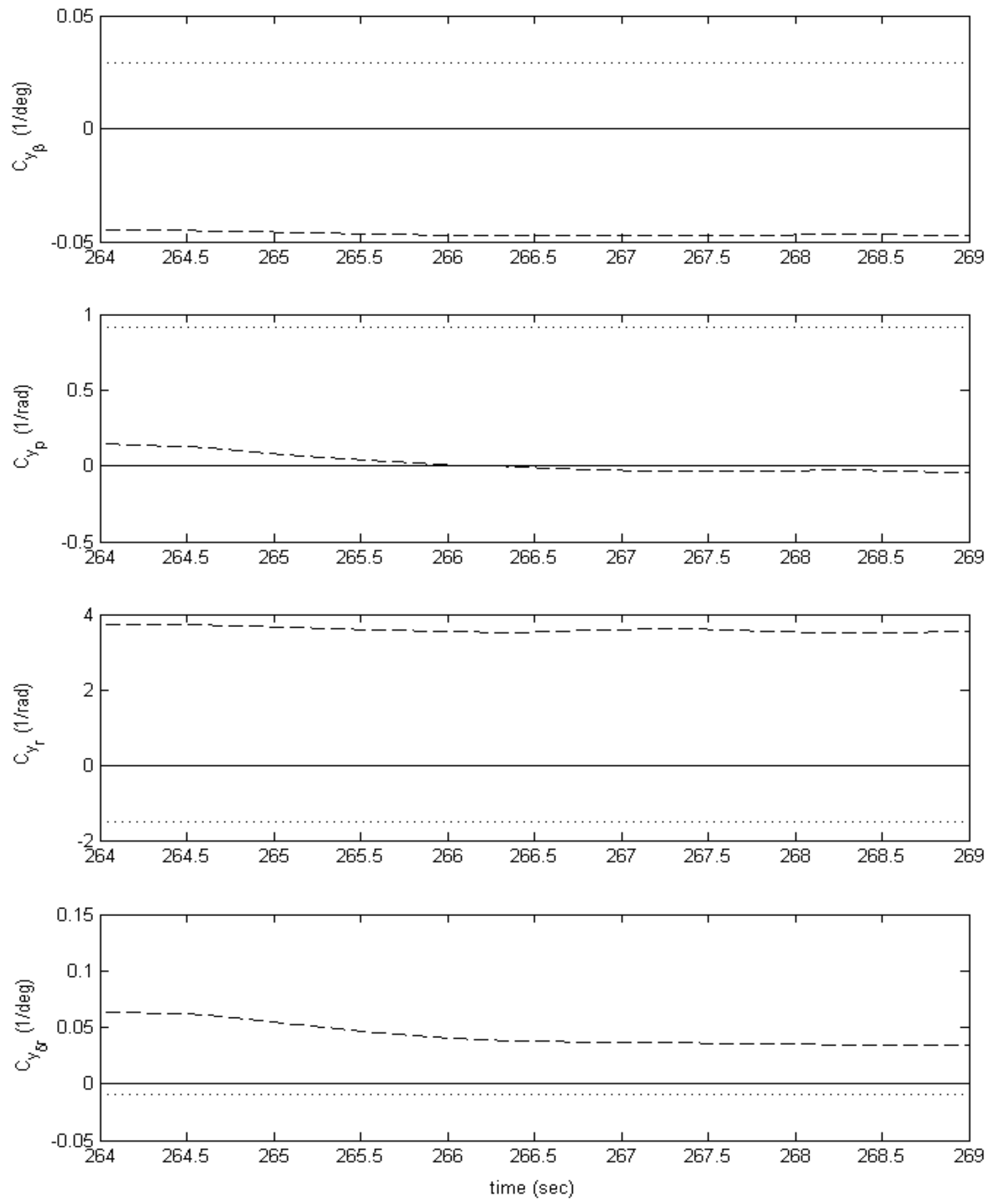

Figure 8.2.11 Comparison of lateral-directional coefficients $C_{y}$ for Flight $D$, Maneuver 3 ( $\delta_{\mathrm{r}}$ input) 
\&, only (Flight E - Man 9)
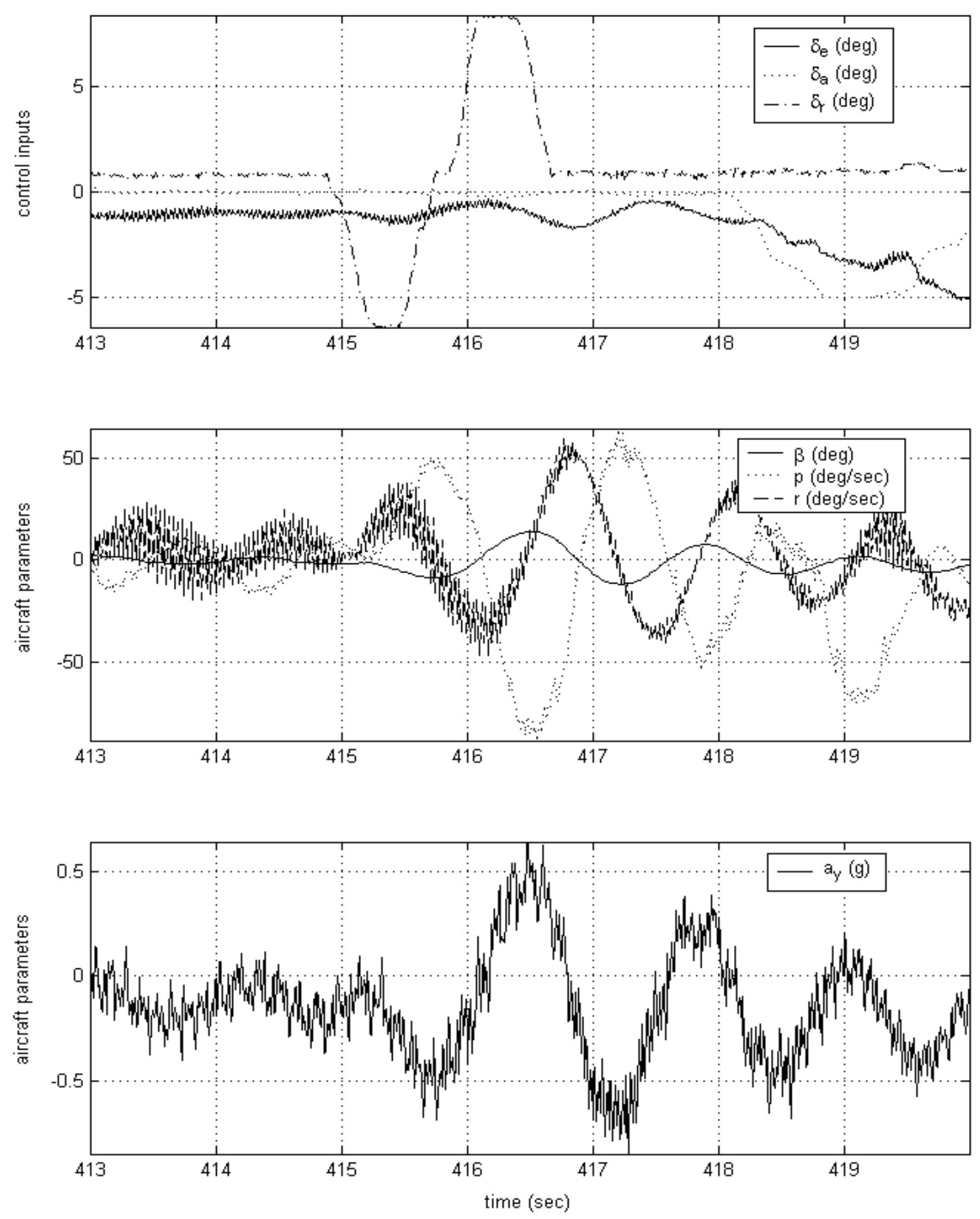

Figure 8.2.12 Measured time histories used for FTR / pEst comparison for Flight E, Maneuver $9\left(\delta_{\mathrm{r}}\right.$ input $)$ 
\& (Flight E - Man 9) - FTR(dash), pEst(dotted)
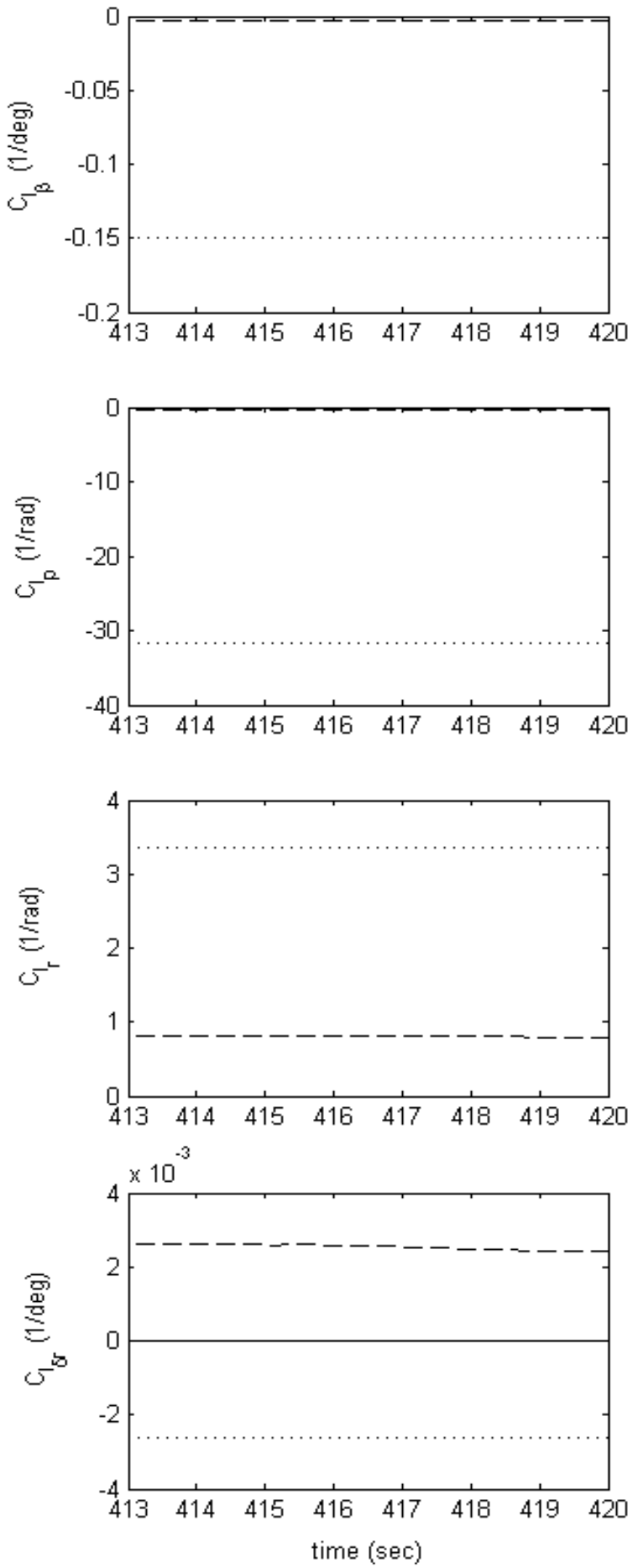

\&) (Flight E - Man 9) - FTR(dash), pEst(dotted)
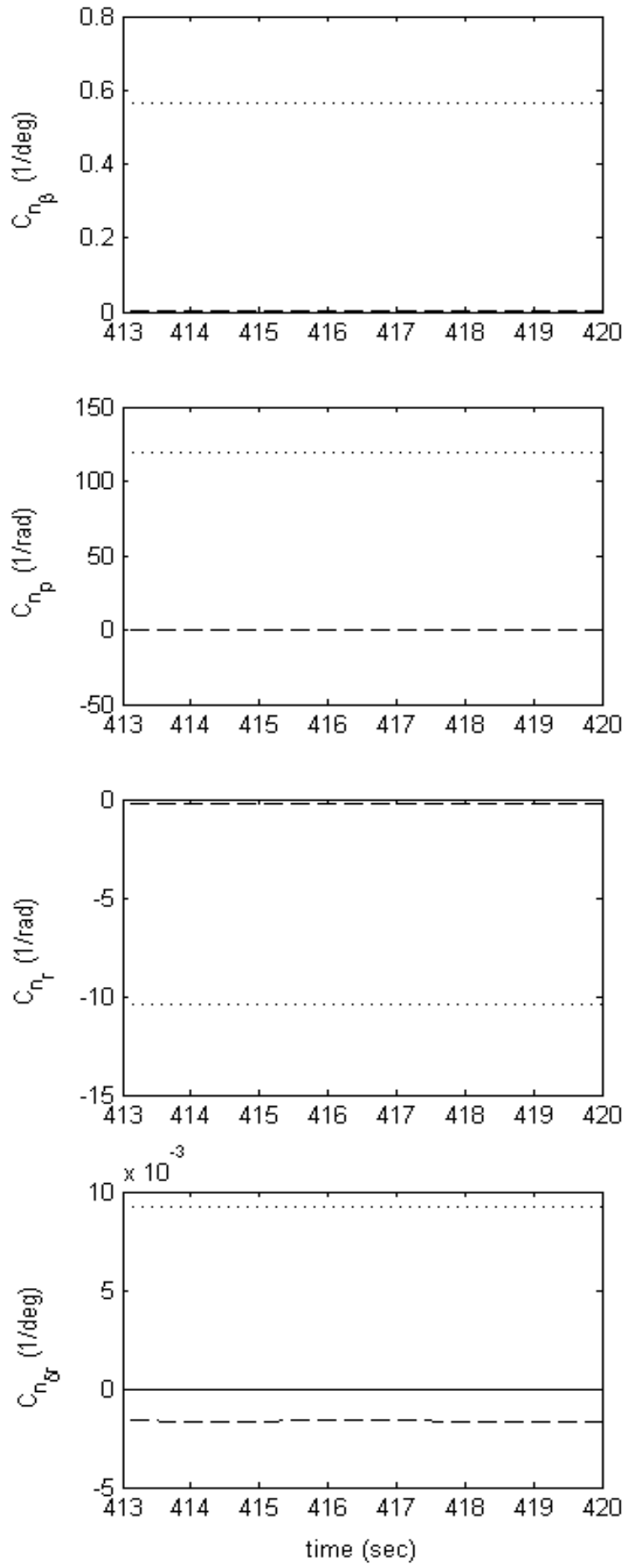

Figure 8.2.13 Comparison of lateral-directional coefficients $C_{1}$ and $C_{n}$ for Flight E, Maneuver 9 ( $\delta_{\mathrm{r}}$ input $)$ 
\&. (Flight E - Man 9) - FTR(dash), pEst(dotted)
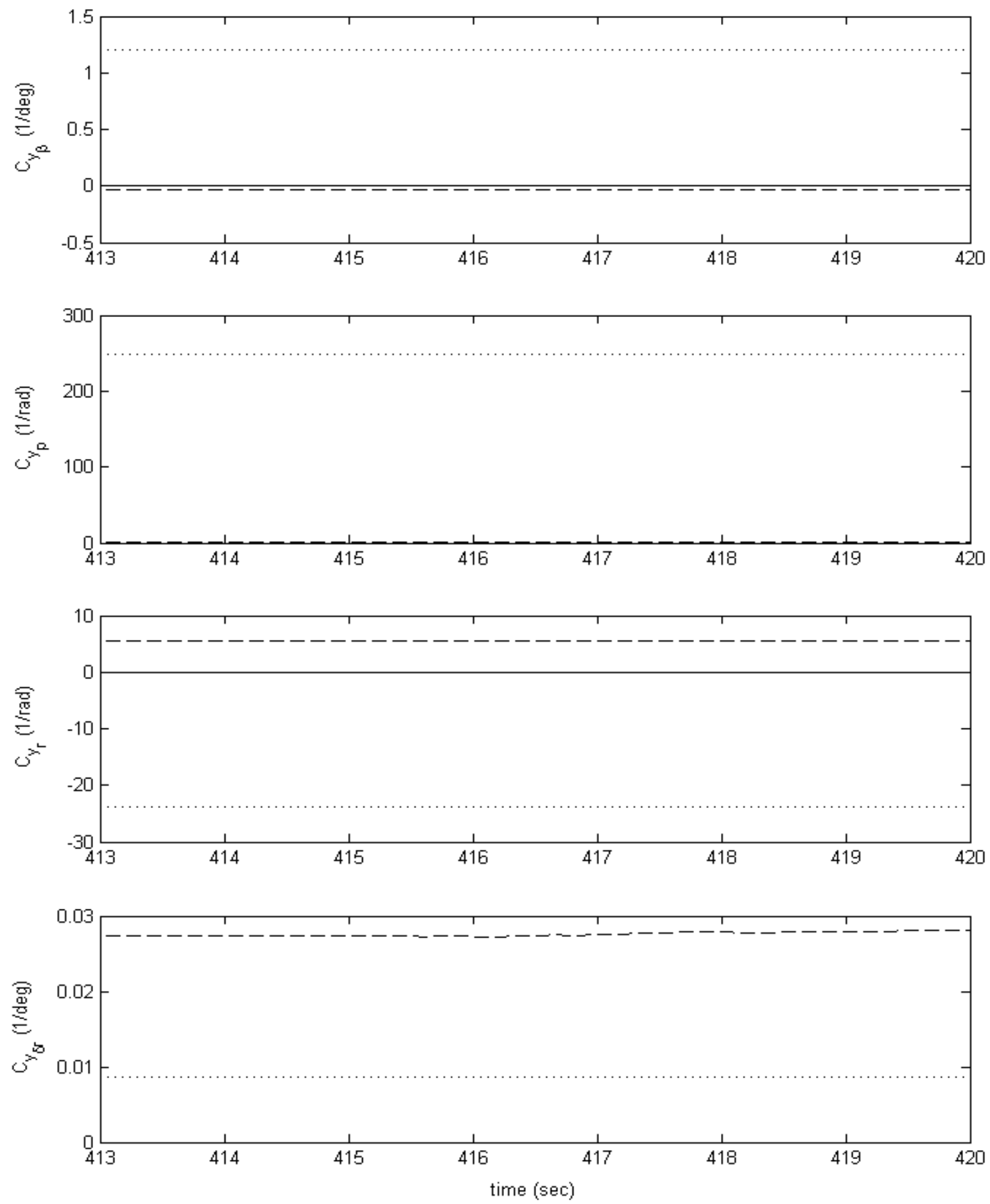

Figure 8.2.14 Comparison of lateral-directional coefficients $C_{y}$ for Flight $E$, Maneuver $9\left(\delta_{\mathrm{r}}\right.$ input $)$ 
$\delta_{\mathrm{a}} \& \delta_{\mathrm{p}}$ inputs (Flight $\mathrm{C}-$ Man 3 )
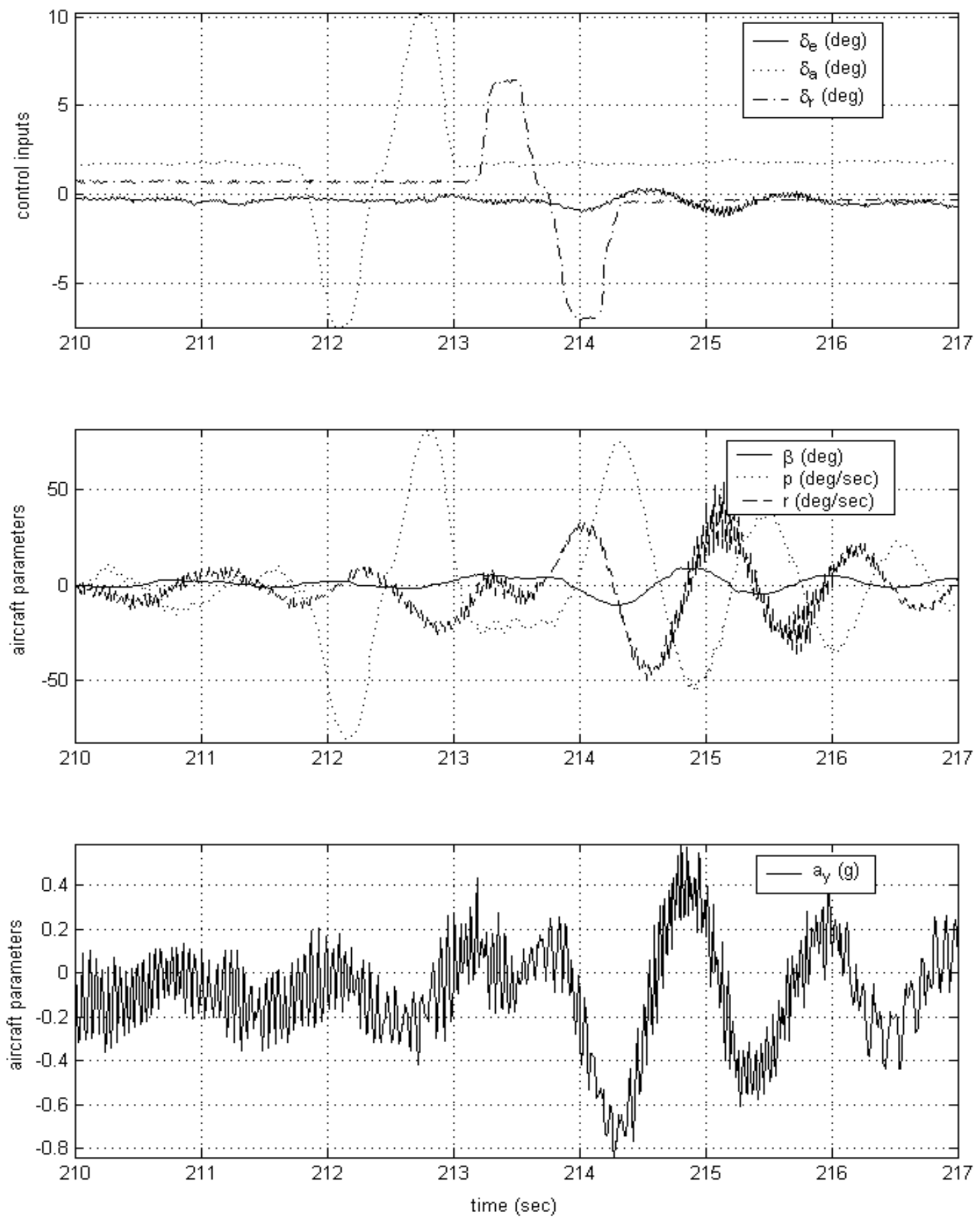

Figure 8.2.15 Measured flight data time histories used for FTR / pEst comparison for Flight $\mathrm{C}$, Maneuver $3\left(\delta_{\mathrm{a}} \& \delta_{\mathrm{r}}\right.$ input combo $)$ 
$\delta_{\mathrm{a}} \& \delta_{\mathrm{r}}$ (Flight $\mathrm{C}-$ Man 3) - FTR(dash), pEst(dotted)
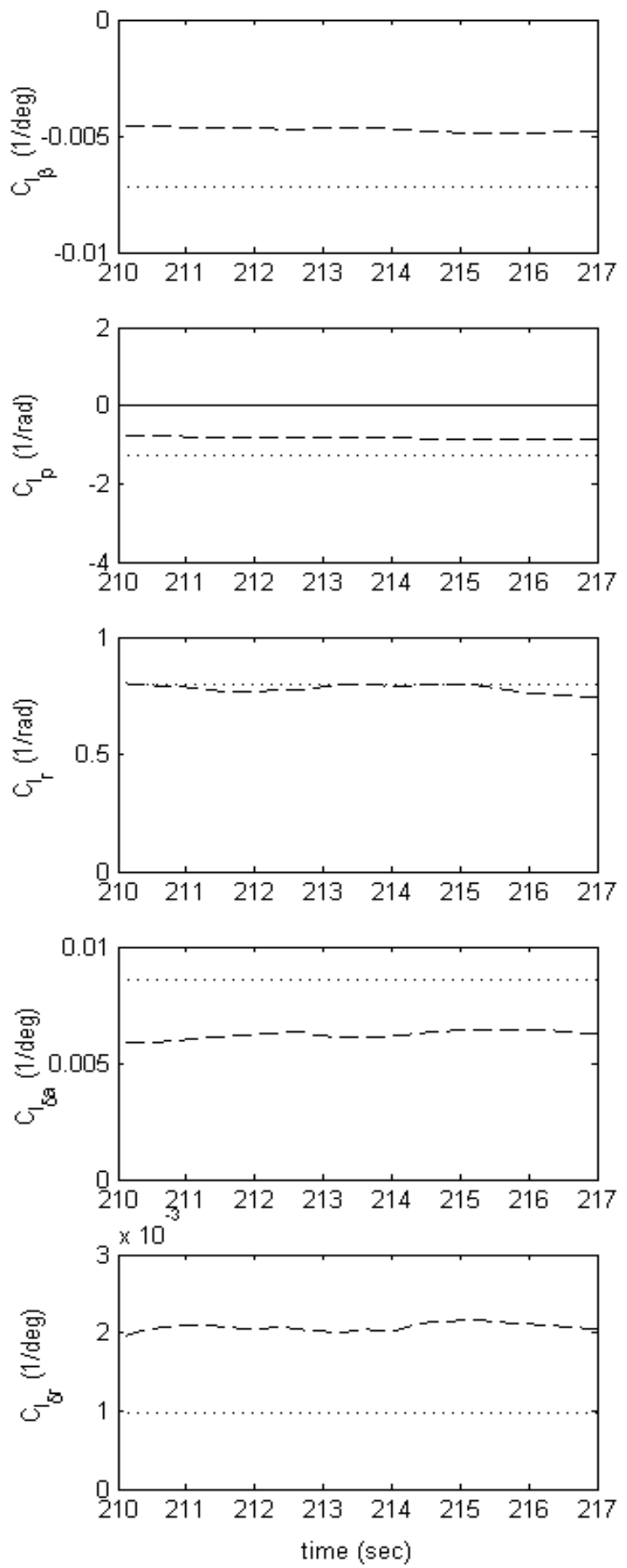

$\delta_{a} \& \delta_{\gamma}$ (Flight C - Man 3) - FTR(dash), pEst(dotted)
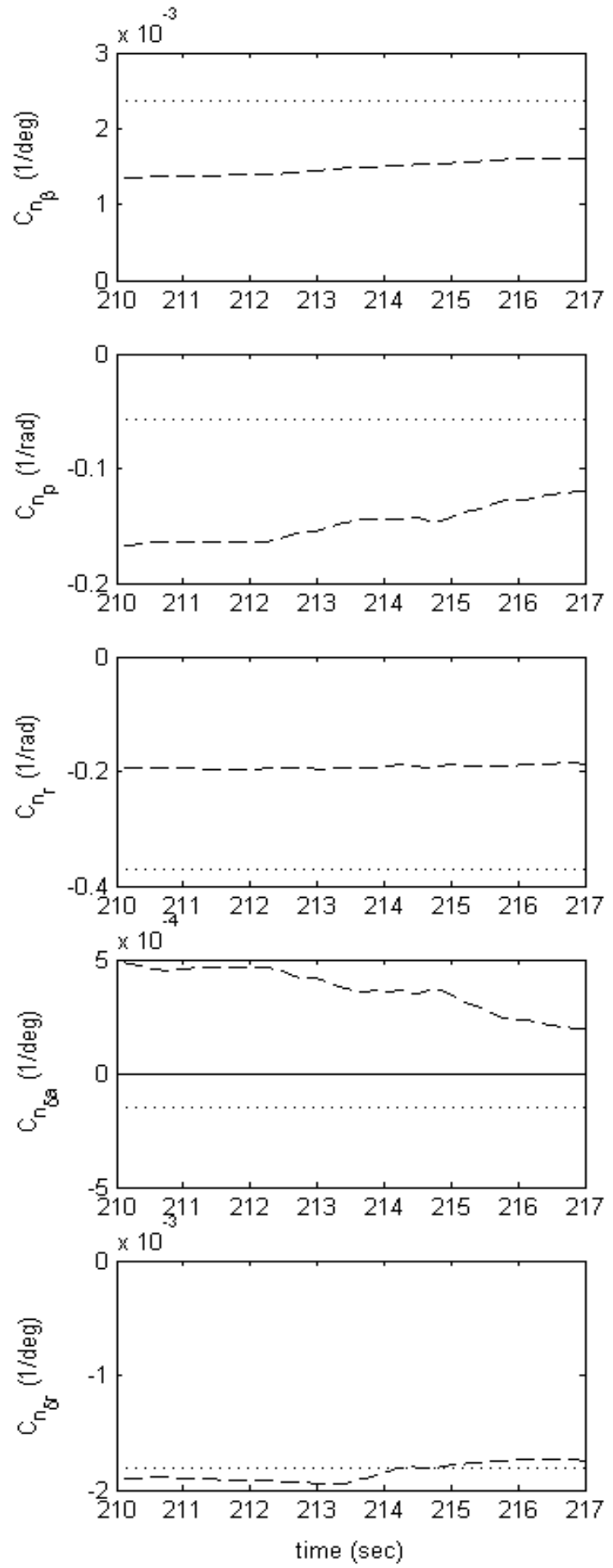

Figure 8.2.16 Comparison of lateral-directional coefficients $C_{1}$ and $C_{n}$ for Flight $\mathrm{C}$, Maneuver $3\left(\delta_{\mathrm{a}} \& \delta_{\mathrm{r}}\right.$ input combo) 
$\delta_{a} \& \delta_{r}$ (Flight C - Man 3) - FTR(dash), pEst(dotted)
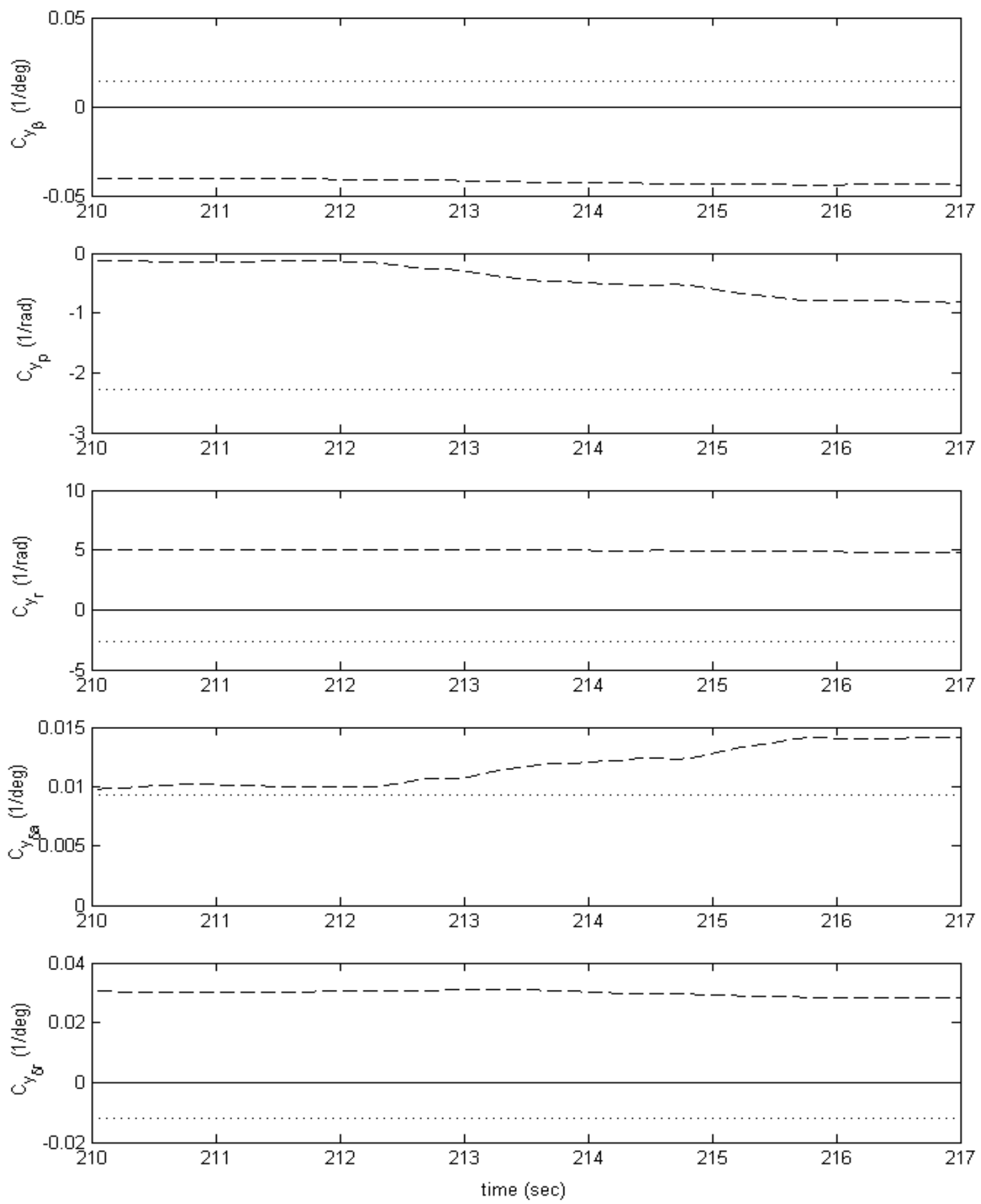

Figure 8.2.17 Comparison of lateral-directional coefficients $\mathrm{C}_{\mathrm{y}}$ for Flight $\mathrm{E}$, Maneuver $3\left(\delta_{\mathrm{a}} \& \delta_{\mathrm{r}}\right.$ input combo $)$ 
$\delta_{\mathrm{a}} \& \delta_{\gamma}$ inputs (Flight $\mathrm{C}-$ Man 6 )
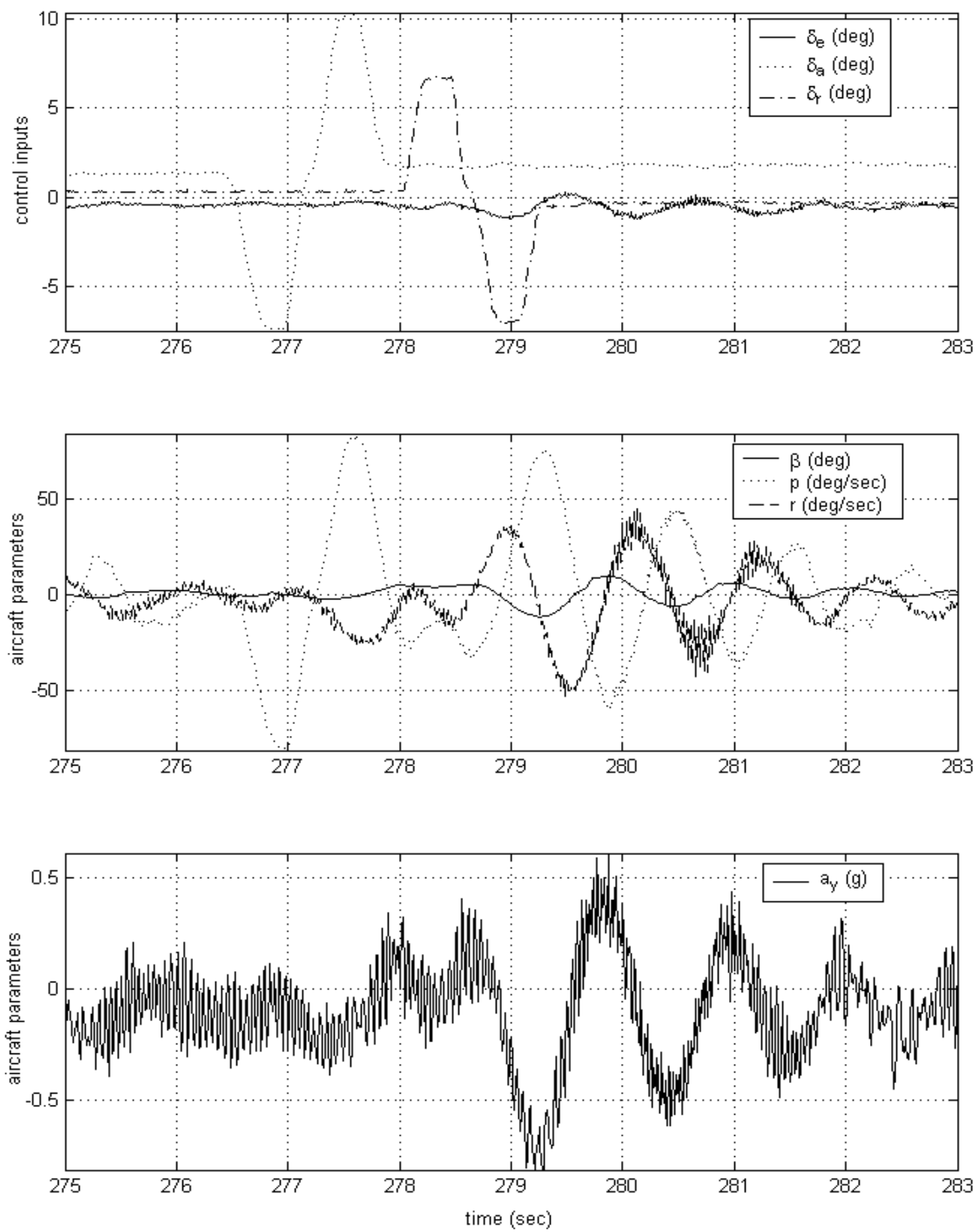

Figure 8.2.18 Measured flight data time histories used for FTR / pEst comparison for Flight $\mathrm{C}$, Maneuver $6\left(\delta_{\mathrm{a}} \& \delta_{\mathrm{r}}\right.$ input combo $)$ 
$\delta_{a} \& \delta_{\text {( }}$ (Flight C - Man 6) - FTR(dash), pEst(dotted)
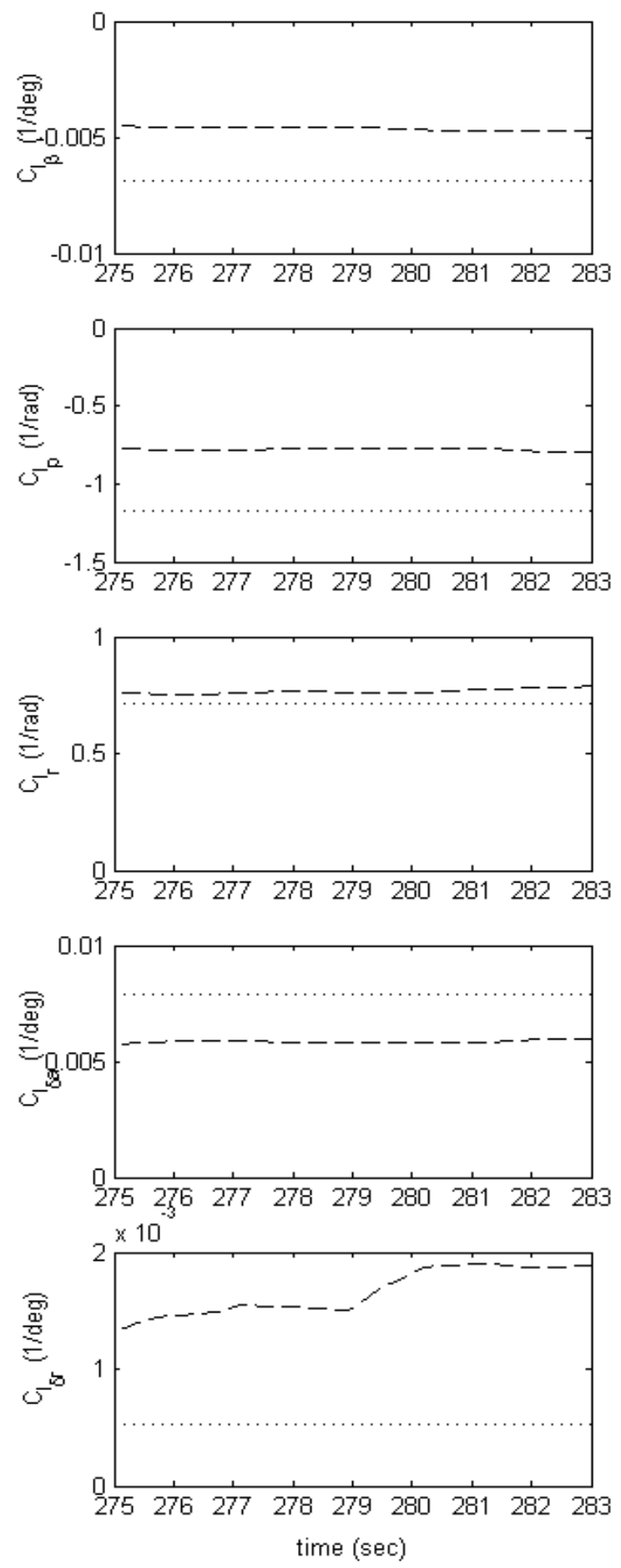

$\delta_{\mathrm{a}} \& \delta_{\mathrm{r}}$ (Flight C - Man 6) - FTR(dash), pEst(dotted)
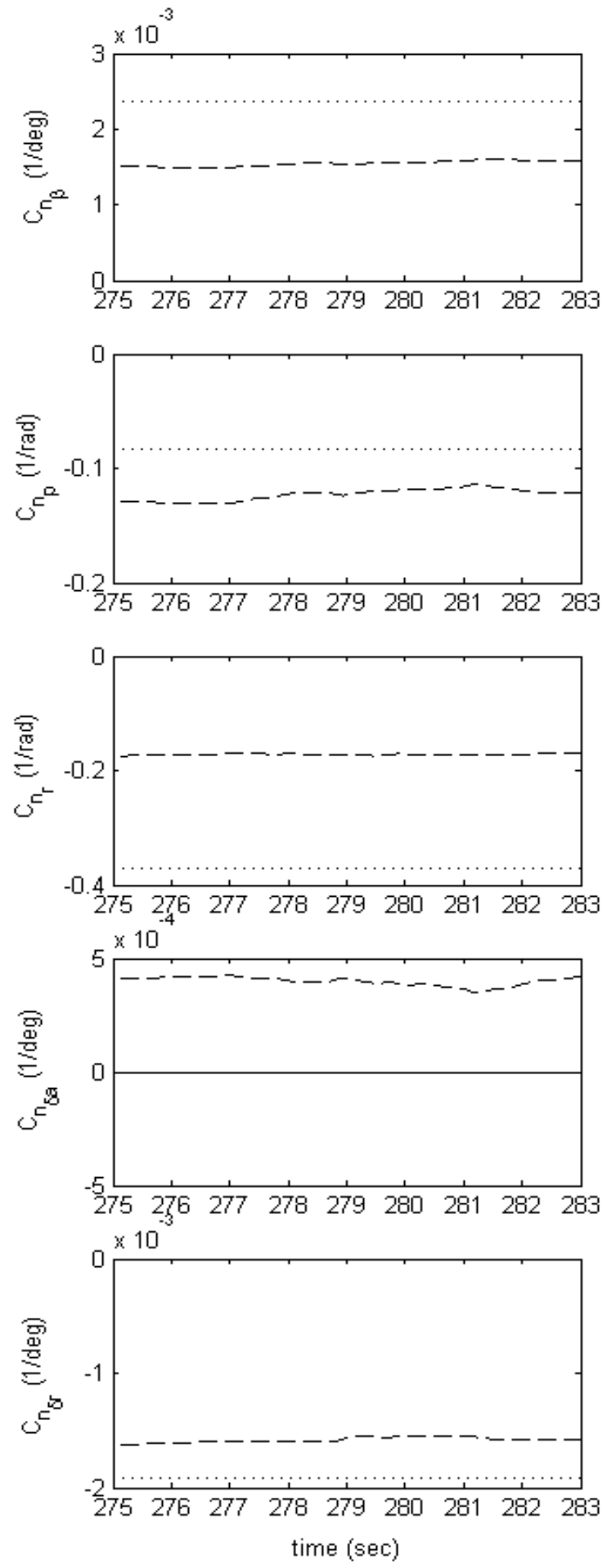

Figure 8.2.19 Comparison of lateral-directional coefficients $C_{1}$ and $C_{n}$ for Flight $\mathrm{C}$, Maneuver $6\left(\delta_{\mathrm{a}} \& \delta_{\mathrm{r}}\right.$ input combo $)$ 
$\delta_{\mathrm{a}} \& \delta_{\mathrm{r}}$ (Flight C - Man 6) - FTR(dash), pEst(dotted)
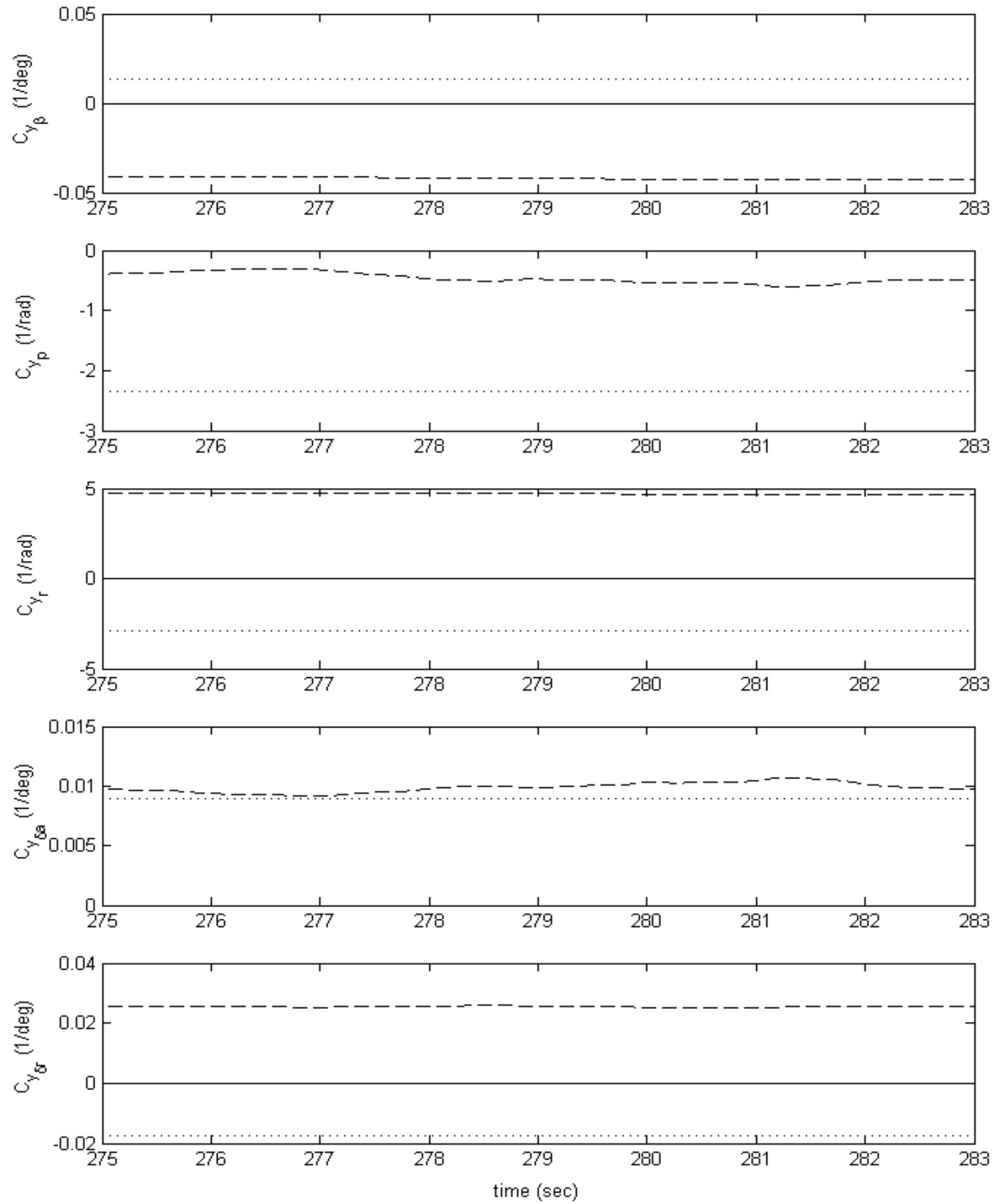

Figure 8.2.20 Comparison of lateral-directional coefficients $\mathrm{C}_{\mathrm{y}}$ for Flight $\mathrm{E}$, Maneuver $6\left(\delta_{\mathrm{a}} \& \delta_{\mathrm{r}}\right.$ input combo $)$ 


\section{Chapter 9}

\section{Conclusions and Recommendations}

\subsection{Conclusions}

The research effort was broken down into four particular phases: one and two involved the development and construction of the aircraft test bed for obtaining flight data which was then used to estimate the longitudinal and lateral-directional stability and control derivatives. From these results, phase three involved an evaluation and verification by using the average phase two estimates to create a linear model, which was then compared with other linear models using other off-line estimation techniques. Phase four compared the pEst estimates with those obtained from an on-line frequency based technique. This work has shown that a reliable RPV could be built, tested, and successfully used for flight testing and PID purposes within an academic setting. In more detail, the experimental RPV was designed and equipped with instrumentation to measure the various flight parameters necessary for a PID analysis. Overall, the aircraft performed well with respect to obtaining the necessary single surface PID inputs required for use in the estimation process. The RPV design was found to be capable of carrying the required instrumentation payload leading into phase two.

Phase two dealt with the collection of the B777 flight testing data and using a parameter estimation program known as pEst which then computed estimates of the aircraft parameters. This estimation software used a Maximum Likelihood estimation technique with a Newton-Raphson minimization scheme. This mathematical model used a traditional static and dynamic derivative buildup. Longitudinal estimates were obtained

for the aerodynamic subcomponents of the total normal force, axial force, and pitching moment coefficients. As for the lateral dynamics, the aerodynamic subcomponents for the total lateral force, rolling moment, and yawing moment coefficients were obtained. A representative CRB for each estimated derivative was shown which gave the standard deviation of the error for that parameter. These estimates were then used to simulate/compute aircraft responses using the actual control inputs. The measured and computed time histories for both longitudinal and later-directional maneuvers were shown to have a good agreement, which indicated a fair representation of the coefficients. 
Longitudinal pitching moment and normal force coefficients showed the aircraft to be statically stable. For the lateral-directional rolling and yawing moment coefficients show the aircraft to be directionally stable. The coefficients obtained were then compared to phase three and four of this work.

In phase three, the average ML estimates obtained from pEst for both the longitudinal and lateral-directional were used to derive a set of linear state variable model which was compared with two other models, specifically, one using a Batch Least Squares technique and the second from the Matlab system identification toolbox. All of the linear models were then compared against the measured flight data. The model based on the BLS approach performed reasonably well against the real flight data, while the models obtained with the subspace-based method from the system identification toolbox and the ML method showed the closest matches against the real measured flight data. A drawback of the system identification toolbox linear model was that the states have no immediate physical interpretation, which can be an important factor when designing control algorithms. The model using the average pEst values, performed very well in the match against the real longitudinal data. Difficulty was found in matching the yaw rate lateral-directional output for the aircraft maneuvers. This is probably due to the noise encountered with the yaw rate measurement in Flight E, which was used for the validation purpose.

Phase four focused on comparing the ML results against the results from another technique based in the frequency domain. Three full sets of flight data were used during the analysis for this phase, in particular, maneuvers from Flights C, D, and E. Both individual and combination control surface maneuvers were evaluated for comparison with specific maneuvers evaluated with the ML method. For the evaluation of the longitudinal maneuver performed in Flight D, the FTR was found to match the pitching moments with the ML estimates. For the lateral data, close comparisons of the rolling and yawing moment coefficients were found. When comparing the individual and combination lateral maneuvers it was found that combination aileron and rudder doublets gave the best FTR results for matching the ML estimates, possibly due to the increase in lateral excitation. 


\subsection{Recommendations}

From this research effort, further work with the B777 aircraft could be pursued in several ways. One suggestion would be to include a feedback key test point within the flight test envelope so that the pilot could try and execute maneuvers at various angles of attack. With the RPV flown visually from the ground, it was difficult to vary any set of tests except to ask the pilot to try and keep the aircraft at a straight and level flight condition for each of the test maneuvers. With respect to the amount of collected flight data, if larger amounts of data were collected there would be a need for an efficient way to catalog the flight parameters. A suggestion would be to create a database that would allow easy access to individual maneuvers. Another suggestion would be to include additional flight parameters such as roll and pitch angle, which would improve the results from the parameter estimation process. A long-term goal could be the implementation of the on-line PID onboard the aircraft in an open loop mode. 


\section{References}

1) Iliff, K.W. and Taylor, L.W., "Determination of Stability Derivatives From Flight Data Using a Newton-Raphson minimization Technique", NASA TN D-6579, 1972

2) Balderson, K.A., "Development of a Digital Matching Aircraft Stability Derivative Estimation Method", Thesis West Virginia University, MAE Dept., Morgantown, WV, 1990

3) Iliff, K.W. and Maine, R.E., "Practical Aspects of Using a Maximum Likelihood Estimation Method to Extract Stability and Control Derivatives From Flight Data", NASA TN D-8209, 1976

4) Iliff, K.W., "Estimation of Aerodynamic Characteristics From Dynamic Flight Test Data", Paper 15 of AGARD CP 235, 1978

5) Iliff, K. W., "Aircraft Parameter Estimation: AIAA Dryden Lecture in Research for 1987”, NASA TM-88281, January 1987

6) Budd, G.D. and Matheny, N.W., "Preliminary Flight Derived Aerodynamic Characteristics of the X-29A Aircraft Using Parameter Identification Techniques", NASA TM-100453, November 1988

7) Seanor, B., "Estimation of the Lateral-Directional Stability and Control Derivatives for the NASA F/A-18 HARV with the Maximum Likelihood Method using Kalviste's Dynamic Modeling", Thesis West Virginia University, MAE Dept., Morgantown, WV, 1995

8) Kalviste, J., "Use of Rotary Balance and Forced Oscillation Test Data in a Six Degrees of Freedom Simulation", AIAA 82-1364, August 1982

9) Kalviste, J., "Coupled Static and Dynamic Stability Parameters", AIAA 89-3362, August 1989

10) Paris, A.C., "Estimation of the Longitudinal and Lateral-Directional Aerodynamic parameters from Flight Data for the NASA F/A-18 HARV", Dissertation WVU, MAE Dept., Morgantown, WV, May 1997

11) Del Frate, J.H., Cosentino, G.B., "Recent Flight Test Experience with Uninhabited Aerial Vehicles at the NASA Dryden Flight Research Center", NASA/TM-1998206546

12) NC State University College of Engineering, “Aerospace Engineering Research Projects 1998-99", January 29, 2002, www.mae.ncsu.edu/programs/grad/aero_research98-99.html 
13) Sydney Univeristy - Unmanned Aricraft Research (RPVs and UAVs), "Unmanned Aerial Vehicle (UAV) Research", January 29, 2002, www.aero.usyd.edu.au/wwwuav/rpb.html

14) Morelli, E. A., "In-Flight System Identification" Proceedings of the 1998 AIAA Atmospheric Flight Mechanics Conference, AIAA Paper 98-4261, Boston, MA, August 1998

15) Morelli, E. A., "Real-Time Parameter Estimation in the Frequency Domain", Proceedings of the 1999 AIAA Atmospheric Flight Mechanics Conference, AIAA Paper 99-4043, Portland, OR, August 1999

16) Morelli, E.A., "High Accuracy Evaluation of the Finite Fourier Transform Using Sampled Data", NASA TM-110340, June 1997

17) Maine, R.E. and Iliff, K.W., "Application of Parameter Estimation to Aircraft Stability and Control; The Output-Error Approach, NASA RF-1168, June 1986

18) Mendel, J.M., "Discrete Techniques of Parameter Estimation", Marcel Dekker, New York, NY, 1973

19) Ljung, L., "System Identification: Theory for the User", Prentice Hall, Englewood Cliffs, NJ, 1987

20) Bodson, M., "An Information-Dependent Data Forgetting Adaptive Algorithm", Proceedings of the 1995 American Control Conference, Seattle, WA, June 1995

21) Monaco, J., Ward, D., Barron, R., Bird, R., "Implementation and Flight Test Assessment of an Adaptive, Reconfigurable Flight Control System", Proceedings of the 1997 AIAA Guidance Navigation and Control Conference, AIAA Paper 97-3738, New Orleans, LA, August 1997

22) Smith, L., Chandler, P.R., Patcher, M., "Regularization Techniques for Real Time Identification of Aircraft Parameters", Proceedings of the 1997 AIAA Guidance Navigation and Control Conference, AIAA Paper 97-3740, New Orleans, LA, August 1997

23) Chandler, P., Patcher, M., Mears, M., "System Identification for Adaptive and Reconfigurable Control", AIAA Journal of Guidance, Control and Dynamics, Vol. 18, No. 3, May-June 1995, pp. 516-524

24) Gelb, A. et al., “Applied Optimal Estimation”, MIT Press, Cambridge, MA, 1973

25) Kirschbaum, H.W., "Estimation of Moments of Inertia of Airplanes from Design Data", NACA TN-575, 1936 
26) Miller, M.P., "An Accurate Method of Measuring the Moments of Inertia of Airplanes:, NACA TN-351, 1930

27) Soule, H.A., Miller, M.P., "The Experimental Determination of the Moments of Inertia of Airplanes", NACA Report No. 467, 1933

28) Roskam, J., Airplane Flight Dynamics and Automatic Flight Controls: Part I, DARcorporation, Lawrence, Kansas,1995

29) Napolitano, M.R., Paris, A., Seanor, B. Bowers, A.H. "Estimation of the Longitudinal Aerodynamic Parameters from Flight Data for the NASA F/A-18 HARV", AIAA Paper 96-3419, Proceedings of the AIAA Atmospheric Flight Mechanics 96 Conference, San Diego, CA, July 1996

30) Napolitano, M.R., Paris, A., Seanor, B. Bowers, A.H. "Estimation of the LateralDirectional Aerodynamic Parameters from Flight Data for the NASA F/A-18 HARV", AIAA Paper 96-3420, Proceedings of the AIAA Atmospheric Flight Mechanics 96 Conference, San Diego, CA, July 1996

31) Maine, R.E. and Iliff, K.W., "The Theory and Practice of Estimating the Accuracy of Dynamic Flight-Determined Coefficients", NASA RP-1077, July 1981

32) Maine, R.E. and Iliff, K.W., "Identification of Dynamic Systems; Theory and Formulation", NASA RP-1138, February 1985

33) Maine, R.E. and Murray, J.E., "pEst Version 2.1 User's Manual", NASA TM-88280, September 1987

34) Maine, R.E, "Manual for GetData Version 3.1 - A FORTRAN Utility Program for Time History Data", NASA TM-88288, October 1987

35) Klein, V. "Aircraft Parameter Estimation in Frequency Domain", Proceedings of the 1978 AIAA Atmospheric Flight Mechanics Conference, AIAA paper 78-1344, Palo Alto, Ca, August 1978

36) Seanor, B., Song, Y., Napolitano, M., Campa, G., "Comparison of On-Line and Off Line Parameter Estimation Techniques Using the NASA F/A-18 HARV Flight Data", AIAA-2001-4261, Atmospheric Flight Mechanics Conference, August 6-9 2001 
Appendix A 


\section{Longitudinal Dimensional Stability Derivatives}

$$
\begin{aligned}
& X_{u}=\frac{-\bar{q}_{1} S\left(c_{D_{U}}+2 c_{D_{1}}\right)}{m U_{1}} \cdot\left(\mathrm{sec}^{-1}\right) \quad X_{T_{u}}=\frac{-\bar{q}_{1} S\left(c_{T_{x u}}+2 c_{T_{x 1}}\right)}{m U_{1}} \cdot\left(\mathrm{sec}^{-1}\right) \\
& X_{\alpha}=\frac{-\bar{q}_{1} S\left(c_{D_{\alpha}}-c_{L_{1}}\right)}{m} \cdot\left(f t \sec ^{-2}\right) \quad X_{\delta_{E}}=\frac{-\bar{q}_{1} S c_{D_{\delta E}}}{m} \cdot\left(f t \sec ^{-2}\right) \\
& Z_{u}=-\frac{\bar{q}_{1} S\left(c_{L_{U}}+2 c_{L_{1}}\right)}{m U_{1}} \cdot\left(\sec ^{-1}\right) \quad Z_{\alpha}=-\frac{\bar{q}_{1} S\left(c_{L_{\alpha}}+c_{D_{1}}\right)}{m} \cdot\left(f t \mathrm{sec}^{-2}\right) \\
& Z_{\alpha}=-\frac{\bar{q}_{1} S \bar{c} c_{L_{\dot{\alpha}}}}{2 m U_{1}} \cdot\left(f t \mathrm{sec}^{-1}\right) \quad Z_{q}=-\frac{\bar{q}_{1} S \bar{c} c_{L_{q}}}{2 m U_{1}} \cdot\left(f t \sec ^{-1}\right) \\
& Z_{\delta_{E}}=\frac{-\bar{q}_{1} S c_{L_{\delta E}}}{m} \cdot\left(f t \sec ^{-2}\right) \\
& M_{u}=\frac{\bar{q}_{1} S \bar{c}\left(c_{m_{U}}+2 c_{m_{1}}\right)}{U_{1} I_{y y}} \cdot\left(f t^{-1} \sec ^{-1}\right) \quad M_{T u}=\frac{\overline{q_{1}} S \bar{c}\left(c_{m_{T U}}+2 c_{m_{T 1}}\right)}{U_{1} I_{y y}} \cdot\left(f t^{-1} \sec ^{-1}\right) \\
& M_{\alpha}=\frac{\bar{q}_{1} S \bar{c} c_{m_{\alpha}}}{I_{y y}} \cdot\left(\sec ^{-2}\right) \quad M_{T \alpha}=\frac{\bar{q}_{1} S \bar{c} c_{m T_{\alpha}}}{I_{y y}} \cdot\left(\sec ^{-2}\right) M_{\dot{\alpha}}=\frac{\bar{q}_{1} S \bar{c} c_{m_{\dot{\alpha}}}}{I_{y y}} \cdot \frac{\bar{c}}{2 U_{1}}\left(\sec ^{-1}\right) \\
& M_{q}=\frac{\bar{q}_{1} S \bar{c} c_{m_{q}}}{I_{y y}} \cdot \frac{\bar{c}}{2 U_{1}}\left(\sec ^{-1}\right) \quad M_{\delta_{E}}=\frac{\overline{q_{1}} S \bar{c} c_{m_{\delta_{E}}}}{I_{y y}} \cdot\left(\sec ^{-2}\right)
\end{aligned}
$$

\section{Modified Longitudinal Dimensional Stability Derivatives}

(used in the State Equations)

$$
\begin{aligned}
& X_{\alpha}^{\prime}=g \cos \gamma_{1}+X_{\alpha} \quad X_{u}^{\prime}=X_{u} \\
& X_{\theta}^{\prime}=-g \cos \gamma_{1} \quad X_{\delta E}^{\prime}=X_{\delta E} \\
& Z_{\alpha}^{\prime}=\frac{\left(g \sin \gamma_{1}+Z_{\alpha}\right)}{\left(U_{1}-Z_{\dot{\alpha}}\right)} \quad Z_{u}^{\prime}=\frac{Z_{u}}{\left(U_{1}-Z_{\dot{\alpha}}\right)} \quad Z_{q}^{\prime}=\frac{\left(U_{1}+Z_{q}\right)}{\left(U_{1}-Z_{\dot{\alpha}}\right)} \\
& Z_{\theta}^{\prime}=\frac{-g \sin \gamma_{1}}{\left(U_{1}-Z_{\dot{\alpha}}\right)} \quad Z_{\delta E}^{\prime}=\frac{Z_{\delta E}}{\left(U_{1}-Z_{\dot{\alpha}}\right)} \\
& M_{\alpha}^{\prime}=M_{\dot{\alpha}} Z_{\alpha}^{\prime}+M_{\alpha} \\
& M_{q}^{\prime}=M_{\dot{\alpha}} Z_{q}^{\prime}+M_{q} \\
& M_{\delta E}^{\prime}=M_{\dot{\alpha}} Z_{\delta E}^{\prime}+M_{\delta E}
\end{aligned} \quad \begin{aligned}
& M_{u}^{\prime}=M_{\dot{\alpha}} Z_{u}^{\prime}+M_{u} Z_{\theta}^{\prime} \\
& M_{\theta}^{\prime}
\end{aligned}
$$




\section{Lateral Directional Dimensional Stability Derivatives}

$$
\begin{aligned}
& Y_{\beta}=\frac{\bar{q}_{1} S c_{Y_{\beta}}}{m} \cdot\left(f t \sec ^{-2}\right) \quad Y_{p}=\frac{\bar{q}_{1} S c_{Y_{p}}}{m} \frac{b}{2 U_{1}} \cdot\left(f t \mathrm{sec}^{-1}\right) \\
& Y_{r}=\frac{\bar{q}_{1} S c_{Y r}}{m} \frac{b}{2 U_{1}} \cdot\left(f t \mathrm{sec}^{-1}\right) \\
& Y_{\delta_{A}}=\frac{\bar{q}_{1} S c_{Y_{\delta A}}}{m} \cdot\left(f t \sec ^{-2}\right) Y_{\delta_{R}}=\frac{\overline{q_{1}} S c_{Y_{\delta R}}}{m} \cdot\left(f t \sec ^{-2}\right) \\
& L_{\beta}=\frac{\bar{q}_{1} S c_{l_{\beta}} b}{I_{x x}} \cdot\left(\sec ^{-2}\right) \quad L_{p}=\frac{\bar{q}_{1} S b c_{l p}}{I_{x x}} \frac{b}{2 U_{1}} \cdot\left(\sec ^{-1}\right) \\
& L_{r}=\frac{\bar{q}_{1} S b c_{l r}}{I_{x x}} \frac{b}{2 U_{1}} \cdot\left(\sec ^{-1}\right) \\
& L_{\delta_{A}}=\frac{\bar{q}_{1} S c_{l_{\delta A}} b}{I_{x x}} \cdot\left(\sec ^{-2}\right) \quad L_{\delta_{R}}=\frac{\bar{q}_{1} S c_{l_{\delta R}} b}{I_{x x}} \cdot\left(\sec ^{-2}\right) \\
& N_{\beta}=\frac{\bar{q}_{1} S c_{n_{\beta}} b}{I_{z z}} \cdot\left(\sec ^{-2}\right) \quad N_{T \beta}=\frac{\bar{q}_{1} S c_{n_{T \beta}} b}{I_{z z}} \cdot\left(\sec ^{-2}\right) N_{p}=\frac{\bar{q}_{1} S b c_{n p}}{I_{z z}} \frac{b}{2 U_{1}} \cdot\left(\sec ^{-1}\right) \\
& N_{r}=\frac{\bar{q}_{1} S b c_{n r}}{I_{z z}} \frac{b}{2 U_{1}} \cdot\left(\sec ^{-1}\right) \quad N_{\delta_{A}}=\frac{\bar{q}_{1} S c_{n_{\delta A}} b}{I_{z z}} \cdot\left(\sec ^{-2}\right) \\
& N_{\delta_{R}}=\frac{\overline{q_{1}} S c_{n_{\delta R}} b}{I_{z z}} \cdot\left(\sec ^{-2}\right)
\end{aligned}
$$




\section{Modified Lateral-Directional Dimensional Stability Derivatives} (used in the State Equations)

Given:

$$
A_{1}=\frac{I_{x z}}{I_{x x}}, B_{1}=\frac{I_{x z}}{I_{z z}}
$$

$Y_{\beta}^{\prime}=\frac{Y_{\beta}}{U_{1}}$

$$
Y_{p}^{\prime}=\frac{Y_{p}}{U_{1}}
$$

$$
Y_{r}^{\prime}=\frac{Y_{r}}{U_{1}}-1
$$$$
Y_{\phi}^{\prime}=\frac{g \cos \Theta_{1}}{U_{1}}
$$

$Y_{\delta A}^{\prime}=\frac{Y_{\delta A}}{U_{1}}$

$$
Y_{\delta R}^{\prime}=\frac{Y_{\delta R}}{U_{1}}
$$

$L_{\beta}^{\prime}=\frac{\left(A_{1} N_{\beta}+L_{\beta}\right)}{\left(1-A_{1} B_{1}\right)}$

$$
L_{p}^{\prime}=\frac{\left(A_{1} N_{p}+L_{p}\right)}{\left(1-A_{1} B_{1}\right)}
$$

$$
L_{r}^{\prime}=\frac{\left(A_{1} N_{r}+L_{r}\right)}{\left(1-A_{1} B_{1}\right)}
$$

$L_{\delta A}^{\prime}=\frac{\left(A_{1} N_{\delta A}+L_{\delta A}\right)}{\left(1-A_{1} B_{1}\right)} L_{\delta R}^{\prime}=\frac{\left(A_{1} N_{\delta R}+L_{\delta R}\right)}{\left(1-A_{1} B_{1}\right)}$

$$
\begin{aligned}
& N_{\beta}^{\prime}=\frac{\left(B_{1} L_{\beta}+N_{\beta}\right)}{\left(1-A_{1} B_{1}\right)} \quad N_{p}^{\prime}=\frac{\left(B_{1} L_{p}+N_{p}\right)}{\left(1-A_{1} B_{1}\right)} \quad N_{r}^{\prime}=\frac{\left(B_{1} L_{r}+N_{r}\right)}{\left(1-A_{1} B_{1}\right)} \\
& N_{\delta A}^{\prime}=\frac{\left(B_{1} L_{\delta A}+N_{\delta A}\right)}{\left(1-A_{1} B_{1}\right)} \quad N_{\delta R}^{\prime}=\frac{\left(B_{1} L_{\delta R}+N_{\delta R}\right)}{\left(1-A_{1} B_{1}\right)}
\end{aligned}
$$

\section{Modified Longitudinal Dimensional Stability Derivatives} (used in the Output Equations)

$$
\begin{array}{lll}
Z_{\alpha}^{\prime \prime}=U_{1} Z_{\alpha}^{\prime}-g \sin \gamma_{1} & Z_{u}^{\prime \prime}=U_{1} Z_{u}^{\prime} & Z_{q}^{\prime \prime}=U_{1}\left(Z_{q}^{\prime}-1\right) \\
Z_{\theta}^{\prime \prime}=U_{1} Z_{\theta}^{\prime}+g \sin \gamma_{1} & Z_{\delta E}^{\prime \prime}=U_{1} Z_{\delta E}^{\prime} &
\end{array}
$$

Modified Lateral Directional Dimensional Stability Derivatives (used in the Output Equations)
$Y_{\beta}^{\prime \prime}=U_{1} Y_{\beta}^{\prime}$
$Y_{p}^{\prime \prime}=U_{1} Y_{p}^{\prime}$
$Y_{r}^{\prime \prime}=U_{1}\left(Y_{r}^{\prime}+1\right)$
$Y_{\phi}^{\prime \prime}=U_{1} Y_{\phi}^{\prime}-g \cos \Theta_{1}$
$Y_{\delta A}^{\prime \prime}=U_{1} Y_{\delta A}^{\prime}$
$Y_{\delta R}^{\prime \prime}=U_{1} Y_{\delta R}^{\prime}$ 